



\section{RECENT ADVANCES IN}

PHYSICAL، AND INORGANIC CHEMISTRY 
BY THE SAME AUTHOR.

RECENT ADVANCES IN ORGANIC CHEMISTRY. With an Introduction by J. NORMAN Collie, Ph.D., 'LL.D., F.R.S. Fourth Edition. 8 vo.

STEREOCHEMISTRY. With 58 Illustrations. Crown 8vo. Second Edition.

-

[Text-Books of Physical Chemistry.

CHEMISTRY AND ITS BORDERLAND. Crown 8 vo.

LONGMANS, GREEN AND CO., LONDON, NEW YORK, BOMBAY, CALCUTTA, AND MADRAS. 
Digitized by the Internet Archive in 2007 with funding from Microsoft Corporation 


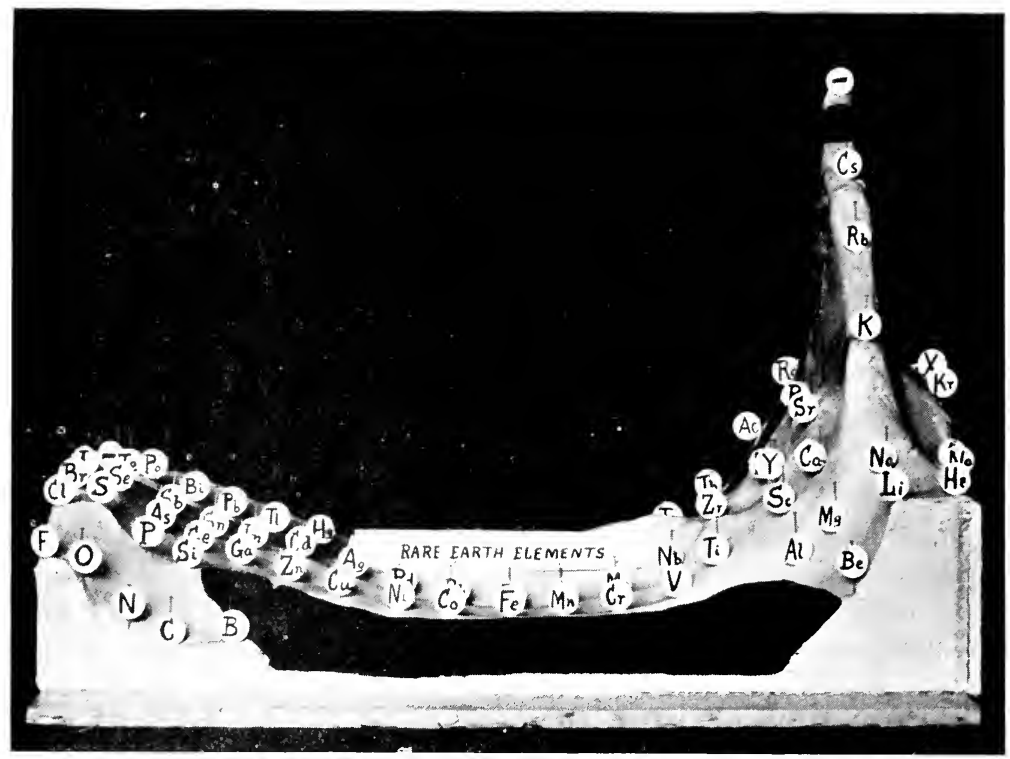

Fis. 23. The Periodic Surface. Front View.

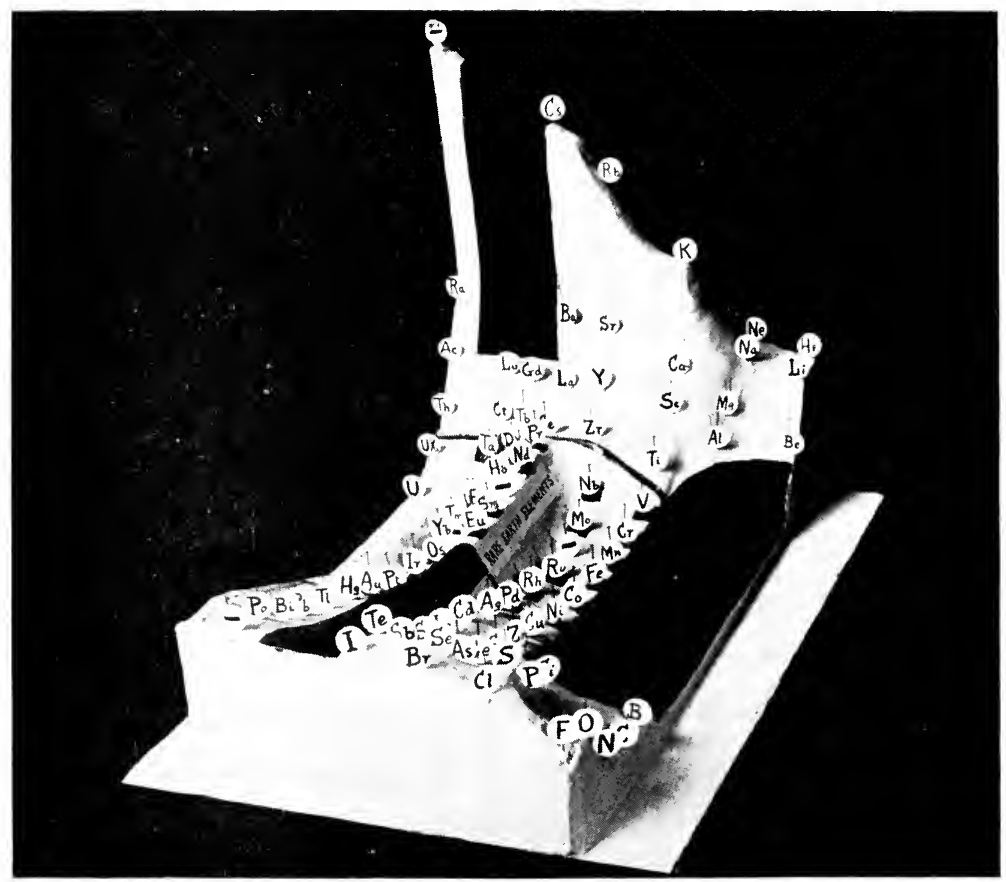

FI(i. 2t. The Periodic Surface. Side View. I'LATE I. 


\section{$\$ 8495 \mathrm{rec}$}

\section{RECENT ADVANCES IN PHYSICAL AND}

\section{INORGANIC CHEMISTRY}

ALFRED W? STEWART, D.Sc.

PROFESSOR OF CHEMISTRY IN THE QUERN'S UNIVERSITY OF BELFASY

WITH AN INTRODUCTION BY

SIR WILLIAM RAMSAY, K.C.B., F.R.S.

WITH TWENTY-FIVE ILLUSTRATIONS

AND A CURVE OF ATOMIC VOLUMES

FOURTH EDITION

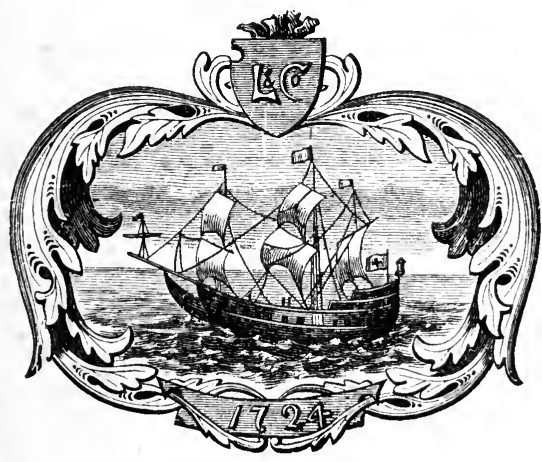

$\frac{160388}{31.3 .21}$

LONGMANS, GREEN AND CO

39 PATERNOSTER ROW, LONDON

FOURTH AVENUE \& 30TH STREET, NEW YORK

BOMBAY, CALCUTTA, AND MADRAS

I 920

All rights reserved. 
<smiles>c1ccc2cc3ccccc3cc2c1</smiles> 


\section{PREFACE TO THE FOURTH EDITION}

IN view of the fact that the last edition of this book was exhausted within little more than a year of its publication, it might be supposed that a fourth edition could have been prepared simply by reprinting the third one; but on account of the important researches which have been carried out within the last twelve months, it was felt that it would be better to rewrite part of the volume rather than to let it go out incomplete. As a result, this edition differs considerably from its predecessor.

Additions have been made to the chapter on absorption spectra; the position of the rare earth elements in the Periodic Table has been more fully examined ; the newer work on massspectra has been described; the problem of transmutation has been given increased space to include the work of Rutherford on nitrogen; and the chapter on the isotopes has been slightly extended.

In view of the recrudescence of active interest in the Periodic Arrangement of the elements, brought about by recent developments in radiochemistry and positive ray analysis, a new chapter has been written on the Periodic Law ; and it is hoped that this may help to direct attention to certain points in that field. Included in this chapter will be found a description of a Periodic Surface, which appears to have certain. advantages over the usual methods of representing the periodicity of elemental properties; and directions for constructing the surface are given in an Appendix. Two modifications of the Atomic Volume Curve are given, each of which seems to present peculiar advantages over the ordinary graph.

These changes have necessitated the recasting of material elsewhere in the book. The chapters on the pseudo-acids and on the inert gases have been deleted, as the subjects are now old and the space was required for other matter. 
I take this opportunity of explaining some of the objects which I have kept before me in the preparation both of this and of previous editions. I have endeavoured to write for the chemist and not for the mathematician. Unless a subject can be treated in such a way that all chemists can understand itwhether they be mathematically inclined or not-I have omitted it from this volume. Thus the applications of the quantum theory, Nernst's heat theorem, and kindred subjects find no place here. It may be objected that to set up such a criterion is to make the work one-sided, and this may be frankly admitted. I believe, however, that from the point of view of the plain chemist, what is lost in this way is gained in others; and I doubt if the inclusion of a few elementary exercises in the calculus would recompense the general chemical reader for the corresponding necessary elimination of some material of morepractical interest. Another reason for the omission of such subjects is the very doubtful value of many of them from the chemical point of view. For example, what application to chemistry has Bohr's view of the atom shown as yet? It has not even satisfied the purely physical requirements of an atomic hypothesis; and, so far as I am aware, its chemical services have been nil. For this reason I have omitted detailed consideration of it, though the mathematics required for its comprehension is extremely elementary.

In conclusion, I wish to thank Professor Collie, F.R.S., Professor Smiles, F.R.S., O.B.E., and Dr. Wright for assistance in the preparation of this edition. My thanks are due also to those reviewers whose criticisms have enabled me to improve the text in certain places.

ALFRED W. STEWART.

The Sir Donald Currie Laboratories, The Queen's University of Belfast. August, 1920. 


\section{EXTRACT FROM \\ PREFACE TO THE THIRD EDITION}

IN the arrangement of the material it seemed best to discard the sharp divisions between inorganic, physical, and radiochemistry, and to deal with the various subjects in an order which obviates as much as possible the assumption that the reader is acquainted with matters described later in the volume. Thus, for example, X-ray spectra are dealt with earlier than the rare earth elements, because Moseley's results define the number of the elements which are still missing and thus enable the position of the rare earth group in the Periodic System to be examined.

No excuse need be offered for the extension of the part of the book devoted to radioactivity, as the recent advances in this region are such as to revolutionise our whole outlook on the fundamental problems of chemistry.

As in previous editions, figures indicate references to the literature, whilst explanatory fcotnotes are marked by asterisks. In this way the reader can avoid unnecessary reference to the foot of the page. The abbreviations employed are mainly those adopted by the Chemical Society in its Journal.

Some readers may detect in the following pages a certain distaste for Physical Chemistry of the kind "Made in Germany." Lest this should be ascribed to a revision of values consequent upon the war, it may be pointed out that exactly similar views, not too obscurely worded, are to be found in previous editions. I have seen no reason to revise my opinions on this subject since 1909, when the first edition was published.

The Physical Chemistry Department,

The University of Glasgow.

October, 1918. 



\section{CONTENTS}

INTRODUCTION $\cdot \quad \cdot \quad \cdot \quad \cdot \quad \cdot \quad \cdot \quad \cdot \quad \cdot \quad \cdot \quad \cdot \quad \cdot \quad$ e

CHAPTEI

I. The Electric Furnace and some of its Products . . 1

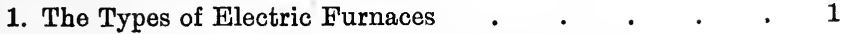

2. The Essentials of Electric Furnace Construction . . 3

3. Graphite • • . • . • • . 5

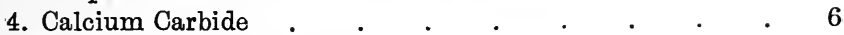

5. Silicon and Silicon Carbide $\quad . \quad$. $\quad . \quad$. $\quad . \quad 8$

6. Silicides $\quad . \quad . \quad . \quad . \quad . \quad . \quad . \quad . \quad 10$

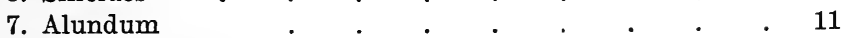

8. Phosphorus . . . . . . . . . . . . 12

9. Carbon Disulphide . $\quad . \quad$. $\quad . \quad$. $\quad . \quad$. 12

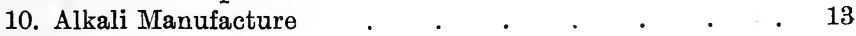

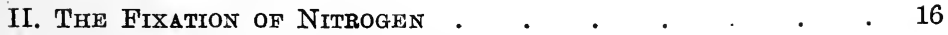

1. The Circulation of Nitrogen in Nature $\quad$. $\quad$ e $\quad . \quad$. 16

2. The Production of Nitrogen Oxides, Nitrous and Nitric Acids . . . . . . . . . . 18

3. The Manufacture of Ammonia . . . . . . . 24

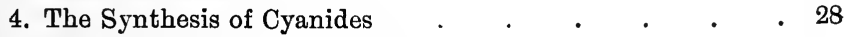

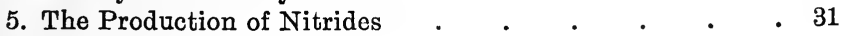

III. The Permutites . . . . . . . . 33

IV. The Peroxides and the Per-acids . . . . . . 37

1. Hydrogen Peroxide $\quad . \quad$. $\quad . \quad$. $\quad . \quad$. $\quad .37$

2. The Metallic Peroxides . $\quad . \quad$. $\quad . \quad$. $\quad . \quad$. 39

3. The General Character of the Per-acids and their Salts . 41

4. The Persulphuric Acids and the Persulphates . . . 42

5. The Per-acids of Group V. $\quad$ • $\quad$ • $\quad$. $\quad . \quad 46$

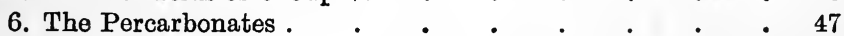

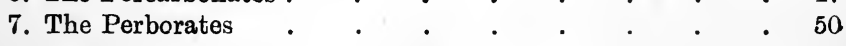


CHAPTER

V. Active Nitrogen . . . . . . . . . . 53

1. The Production of Active Nitrogen . . . . . 53

2. The Properties of Active Nitrogen . . . . . $\quad 54$

3. The Influence of Impurities . $\quad$. $\quad . \quad$. $\quad . \quad 55$

4. The Effect of External Conditions upon Active Nitrogen . $\quad 57$

5. A Third Form of Nitrogen . $\quad$. $\quad . \quad$. $\quad . \quad 58$

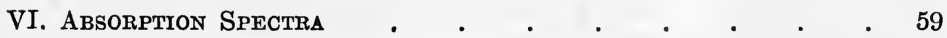

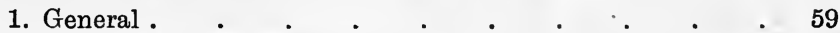

2. The Hartley Method . $\quad$. $\quad$. $\quad . \quad . \quad .66$

3. The Problem of General Absorption $\quad$ - . . $\quad 67$

4. The Factors affecting Selective Absorption . . . 76

5. Valency and Absorptive Power $\quad$. . . $\quad$. $\quad . \quad 58$

6. The Spectroscopic Determination of Chemical Change $\quad 83$

7. The Spectrophotometer . . . . . . . 84

8. The Calculation of Absorption Curves . $\quad$ - 86

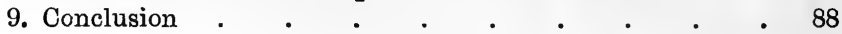

VII. X-rays ANd some Atomic Properties . . . . 90

1. The Phenomena of Crookes' Tubes . . . . 90

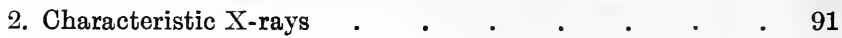

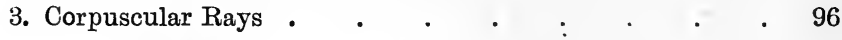

VIII. X-rays and Crystal Structure $\quad$. $\quad . \quad$. $\quad . \quad 98$

1. The Phenomena of Diffraction . . . . . $\quad .98$

2. Space Lattices . $\quad . \quad$. $\quad . \quad$. $\quad . \quad 102$

3. The Crystal as a Diffraction Grating . . . . 104

4. Laue's Experiment . . . . . . . . 105

5. The X-ray Spectrometer . $\quad$. $\quad . \quad$. $\quad . \quad 106$

6. The Analysis of Crystal Structure . $\quad . \quad$. $\quad . \quad 107$

IX. X-ray Spectra and Atomic Numbers . . . . . . $\quad .114$

1. The Method of Measuring X-ray Spectra . . . . 114

2. The Nature of the X-ray Spectra . . . . . . 115

3. The Gaps in the Periodic Table . . . . . . 118

4. The Anomalies of the Periodic Table . . . . . 119

5. The Spectrum of Hydrogen . . . . . . . 120

6. Molecular Numbers . . . . . . . . . . 121

7. Conclusion . . . . . . . . . . . . 122

X. The Elements of the Rare Earths . . . . . 124

1. Introductory • . . . . . . . . . 124

2. The Physical Properties of the Rare Earth Elements . 126

3. The General Chemical Character of the Rare Earth Elements . . . . . . . . . 126

4. Methods of Purifying the Rare Earths . . . . 128

5. Industrial Applications of the Rare Earth Elements 133 
XI. Incandescent Gas Mantles . $\quad . \quad \ldots \quad$. $\quad . \quad$. $\quad . \quad 135$

1. Introductory . . . . . . . . . . 135

2. The Treatment of Monazite Sand . . . . . . 136

3. Fabrics and their Treatment . . . . . . . . 138

4. Impregnation, Fixing, and Branding $\quad$ - $\quad$. $\quad . \quad$. 138

5. Burning, Collodionizing, and Testing . . . . 140

XII. Positive Ray Analysis and Mass Spectra • . . 141

1. The Apparatus .

2. The Theory of the Method . $\quad . \quad$. $\quad . \quad . \quad . \quad 143$

3. The Interpretation of the Photographs . . . . . 145

4. The Substance $\mathrm{X}_{3}$.

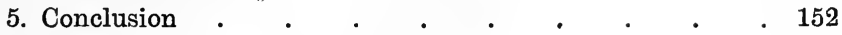

XIII. The Phenomena of Radioactivity . $\quad$. . . . 154

1. Historical . . . . . . . . . . 154

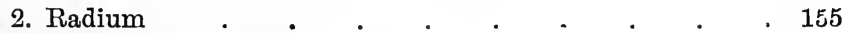

3. The Becquerel Rays . $\quad$. $\quad$. $\quad$. $\quad$. $\quad$. 158

XIV. The Disintegration Theory and the Radioactive Series . 166

1. The Disintegration Theory $\quad$. $\quad . \quad$. $\quad$. 166

2. Multiple Disintegration . . . . . . . . 169

3. The Radioactive Series . $\quad . \quad$. $\quad . \quad$. $\quad . \quad$. 170

4. Potassium and Rubidium . . . . . . 174

5. Radioactive Recoil . . . . . . . . . . . 174

6. The Geiger-Nuttall Relation . . . . . . 175

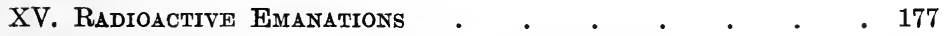

1. The Emanations of Thorium and Actinium . $\quad . \quad . \quad 177$

2. Niton . . . . . . . . . . 179

3. The Production of Helium $\quad$. $\quad$. $\quad$. $\quad . \quad 187$

4. The Disintegration Theory and the Age of Minerals . $\quad 189$

XVI. Artificial Transmutation . . . . . . . . 191

1. Introductory . $\quad . \quad$. $\quad . \quad$. $\quad . \quad$. $\quad .191$

2. The Work of Ramsay and Cameron _ . . . . 191

3. The Investigations of Collie and Patterson . . . $\quad . \quad 193$

4. Rutherford's Experiments . . . . . . . $\quad . \quad 196$

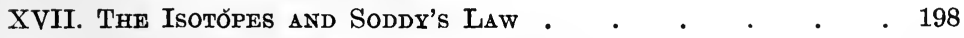

1. Soddy's Theory of Isotopic Elements . . . . . 198

2. The $a$-Ray Change . . . . . . . . 201

3. Fleck's Investigations of the Chemistry of the Radioelements . . . . . . . . . 202

4. The $\beta$-Ray Change . $\quad . \quad$. $\quad . \quad$. $\quad . \quad$. $\quad . \quad 204$

5. Soddy's Law . $\quad . \quad$. $\quad . \quad$. $\quad . \quad$. $\quad .205$

6. $\beta$-Rays and Ionic Charges . . . . . . . 208

7. The Atomic Weight of Lead . . . . . . $\quad . \quad 209$

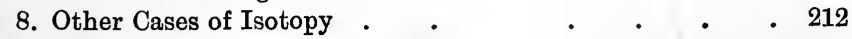

9. Conclusion . . . . . . . . . . . . 214 
XVIII. The Elements . . . . . . . . . . 218

1. Isotopes and Isobares . $\quad . \quad$. $\quad . \quad$. 218

2. The Structure of the Atom . $\quad$. $\quad$. $\quad$. $\quad . \quad 225$

3. The Problem of Atomic Weight $\quad$. $\quad . \quad$. $\quad . \quad 234$

XIX. The Periodic Law $\quad$. $\quad . \quad$. $\quad . \quad . \quad . \quad . \quad 236$

1. Mendeléeff, Lothar Meyer and Crookes . . . . 236

2. The Periodic Table and the Atomic Volume Curve . . 239

3. Spiral Arrangements of the Elements . . . $\quad 250$

4. The Periodic Surface . . . . . . . . 251

5. The Problem of the Rare Earth Elements . . . 253

6. The Theory of Meta-elements . . . . . . . 261

7. Conclusion . . . . . . . . . . 263

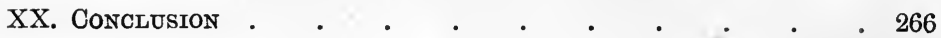

Appendix. Notes on the Construction of the Periodic Surface . . 275

NAME INdex . . . . . . . . . . . . . 277

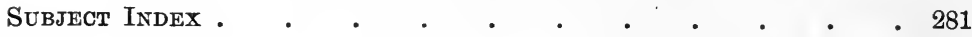




\section{IN'TRODUCTION}

AT all periods of the world's history certain problems have impressed themselves on men's minds as being of paramount importance. History affords many examples of this. From the doctrine of "the divine right of kings" to the question of "woman's suffrage" is a long step, but not a longer.one than from the once all-absorbing theme which exercised the minds of men of science as regards the true nature of phlogiston to the present contest regarding the structure of the cobaltammines. Science has, however, this advantage over politics-that experiments devised to decide knotty questions are more easily carried out; and, further, that it is in the interest of no one to conceal the truth.

In Dr. Stewart's Recent Advances in Physical and Inorganic Chemistry, he has treated of a number of subjects which are at present prominent in the minds of chemists. No doubt they will in time be succeeded by others equally engrossing; the essential features of the subjects here considered will have been established or refuted, and new views will in some cases succeed those which he has expounded. Such new views, however, will be the result of maturer knowledge, gained by incessant experiment. At present these essays represent the state of knowledge which we possess; and rival theories have been set forth with fairness, and yet not without some guidance, from the author of this work, who has not scrupled to express his own opinion where he holds a decided view.

The enormous mass of chemical literature which floods our tables monthly makes it impossible to do more than glance at the titles of the papers; and each of these, it is sometimes almost sad to think, represents much patient and careful work which ought to receive, at all events, some consideration. The 
chemically educated public, too, is rapidly increasing; those who are not themselves actively engaged in furthering the science, and who have neither the leisure nor inclination to sit down and read the Transactions of the Chemical Society or the Zeitschrift fiir anorganische Chemie, are the better for having their chemical food prepared for the table instead of trying to assimilate indigestible masses of what is often very crude material. Dr. Stewart may be likened to a skilful cook, who has trimmed his joint, rejecting all innutritious and redundant excrescences, and has served it up to table in a palatable form Such essays, I venture to think, will do more to encourage a taste for chemistry than many text-books. They will be followed with pleasure by any one who has mastered the nomen. clature and is at home with the simpler conceptions of chemistry. Each may be taken to represent in a readable form the latest work on the subject of which it treats.

The book, it may be hoped, will have a still further use it cannot be doubtful that some who peruse it will have suggested to them various directions in which they may profitably attempt to increase knowledge. Nothing is so sac as to see much time and labour spent, with patience anc devotion, in the investigation of some matter which possesses no real importance. It may be retorted that every true statement is of importance, but this is not so. It is only statements which hold forth some prospect of contributing to an organic whole which can be held valuable. There may, perhaps, be a little more merit in ascertaining to the hundredth of a degree the boiling-point of sulphur than of measuring the area of the wings of some particular butterfly but the difference is barely appreciable. One is as likely to prove useless as the other. It would be well if enthusiast anxious to carry on research would remember that it is much more stimulating to carry on an interesting than an uninteresting research. It is, I believe, not improbable that the reading of such a work as this may aid those imbued with the spirit of investigation to make a happy choice of a subject of research. Should this hope be realised, Dr. Stewart will have done a most useful work. 


\section{CHAPTER I}

\section{THE ELECTRIC FURNACE AND SOME OF ITS PRODUCTS}

\section{The Types of Electric Furnaces.}

Though the suggestion of an electric furnace dates from Pichon in 1853, the first practical model was constructed by Siemens in 1878, with the object of fusing refractory metals and their ores. In 1885 the Cowles furnace made its appearance, initially with a view to the reduction of oxides. Moissan, ${ }^{1}$ in 1893 , carried out a series of investigations on the action of the electric furnace upon various materials, and to him we owe much of our knowledge of the behaviour of substances at high temperatures. The carbides, borides, and silicides were placed by his work among the class of readily obtainable compounds. Since that time, the application of the electric furnace to scientific and commercial problems has become wider; and at the present day the electric furnace industry has its ramifications through the most diverse fields.

Naturally, when the instrument has been applied to so many different problems, the apparatus has required modification in many respects; and at this point it may be well to give a brief survey of the chief types which are in use.

Speaking generally, electric furnaces may be divided into two main classes: arc furnaces and resistance furnaces. In the arc furnace, the heat is derived from an electric arc which plays in the furnace; whilst in the resistance furnace the heat is generated in a resistance through which the current runs, and from this resistance the heat passes to the charge in the furnace by conduction.

1. Arc Furnaces.-This group of furnaces may be divided into two sub-groups: furnaces with an independent arc, like 
Moissan's ; and furnaces in which the arc exerts a direct heating effect by coming in contact with the charge. In the independent arc furnace, the arc plays between two electrodes placed above the surface of the charge; and the heat is conveyed to the furnace contents by radiation, either direct or by reflection from the walls of the furnace. In the direct heating arc type two possibilities present themselves. The Girod furnace has one electrode buried in the furnace contents; so that the arc plays from the upper electrode on to the surface of the charge. In the Heroult double arc furnace this effect is increased by placing both electrodes above the furnace contents, but so far removed from one another that no arcing is possible between them. The result of this is that in order to pass the current from one electrode to the other it is necessary to form an arc between the positive electrode and the charge and another arc between the charge and the negative electrode. Thus two arcs, one from each electrode, play upon the surface of the charge, which is included in the electric circuit.

2. Resistance Furnaces.-Here also we have two main types: those furnaces which are furnished with a special resistance; and those which depend for the resistance upon the contents of the furnace. It will be advisable to deal with each class separately.

In the first type it is possible to imagine two variations: for we might place the resistance which is to generate the heat either in the walls of the furnace or in the middle of the furnace contents. In the ordinary laboratory electric tube furnace the first method is adopted, the resistance employed being a ribbon of platinum or other metallic material coiled around the porcelain tube which forms the actual furnace wherein refractory materials are placed. In the carborundum furnace, on the other hand, the resistance is built of carbon which becomes hot on the passage of the current; and this carbon resistance is imbedded in the furnace charge, which is thus heated by conduction from the resistance.

Turning now to the second type of resistance furnace, viz., that in which no specially constructed resistance is used, it is clear that here also a number of modifications may be expected. In the first place, we may have furnaces devised purely for heating effect; whilst in the second place, we may have furnaces 
in which both heating effect and electrolytic action come into play. In the case of purely heating furnaces, again, the nature of the furnace will differ in detail according as to whether the furnace contents are solid, semi-fluid, or liquid during the process.

The following table ${ }^{1}$ will give at a glance the relations between the different types of construction :-
Arc Furnaces $\left\{\begin{array}{l}\text { Independent arc } \\ \text { Direct heating arc }\end{array}\right.$
$\left\{\begin{array}{l}\text { Single arc } \\ \text { Double arc }\end{array}\right.$

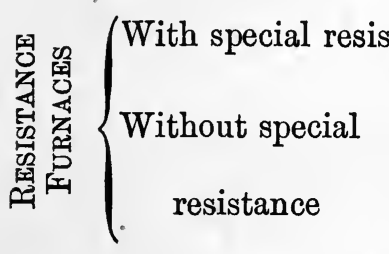
Resistance in walls Resistance in charge

It is unnecessary to describe the various types in detail at this point, as the most important differences will be referred to in connection with the principal products of the electric furnace industry. In the remaining portion of this chapter the nonmetallurgical applications of the electric furnace will be chiefly dwelt upon; as the metallurgical side would occupy more space than can be spared in this volume. ${ }^{2}$

\section{The Essentials of Electric Furnace Construction.}

Reduced to its simplest terms, an electric furnace comprises three factors: (1) a source of current; (2) electrodes and their holders; (3) a refractory casing.

With regard to the current, this is usually supplied by a dynamo coupled with a transformer to reduce the voltage. Generally an alternating current is used; but in the case of processes involving electrolytic action a direct current is necessary. Naturally the electric furnace industry tends to centre in districts like Niagara where water-power is cheap and current

1 Modified from Stansfield, The Electric Furnace.

2 For details on this part of the subject, see Stansfield, The Electric Furnace (1914); Wright, Electric Furnaces and their Industrial Applications (1910); and Rideal, Electrometallurgy (1918). 
can be obtained economically. It is unnecessary to give any figures with regard to quantities of current employed, since these vary from process to process.

The electrodes usually employed in electric furnaces are carbon rods, for no other material is capable of standing the high temperatures which can be obtained. In certain types of furnace actual electrodes are not necessary, the current in such cases being generated in the furnace by induction. Provision is sometimes necessary to avoid the external ends of the electrodes becoming too hot. Connection between the electrodes and the cables carrying the current is made by means of metal clamps which also act as electrode holders.

We now come to one of the most important points in an electric furnace: the materials of which the furnace casing is composed. It must be remembered that the temperature of the positive crater of an arc playing between carbon rods is about $3500^{\circ} \mathrm{C}$., so it is clear that any material which is to be brought near such a focus must be of the most infusible type. In one of Moissan's experiments his furnace, which was built of quicklime, was destroyed in a few minutes: the quicklime melted and ran like water; and a portion of it was even volatilized.

Nor is it always sufficient merely to choose a refractory material for the furnace walls; for it may be that the furnace charge, under the action of heat, may act upon the furnace lining, causing great deterioration.

Amongst the most suitable materials for furnace linings are the following. Fireclay bricks will stand a temperature of $1600^{\circ}$ C., but they are rapidly attacked by slags containing metallic oxides. The same defect is noticeable in silica bricks, but these will remain unfused at $1700^{\circ} \mathrm{C}$ : Lime and magnesia will stand temperatures above $2000^{\circ}$ C., but they are both crumbly materials which are apt to give trouble. On the other hand, they do not form fluxes with metallic slags. Chrome iron ore (chromite) is a neutral refractory material which is not easily acted on and stands temperatures up to $2100^{\circ} \mathrm{C}$. Alundum is almost as infusible. Carbon itself is, of course, the most infusible material of all; but it suffers from the disadvantage that it is readily oxidized at high temperatures, so its use is restricted. Zirconia is also utilized. 
It is sometimes found convenient to construct the furnace wall in two layers, the front layer being refractory but not completely non-conducting, whilst behind it is placed a layer of some material of lower conductivity which prevents loss of heat from the furnace.

In connection with the furnace construction, another point may be mentioned. Some furnaces are designed to run with a single charge; and at the end of the process the furnace is allowed to cool and the charge is withdrawn. Other types have been devised which permit of continuous action, the finished material being withdrawn and fresh charges being introduced without any interruption in the action of the. furnace. Finally, by using a revolving furnace, it is possible to combine the advantages of both these types: the arc is kept continually at work while raw material is being added at a second region of the furnace, and the finished product is being cooled and extracted at yet a third point.

\section{Graphite.}

The action of the electric furnace upon carbon was studied by Moissan, who found that under suitable conditions amorphous carbon could be transformed into either graphite or diamond. By dissolving carbon in molten iron in the electric furnace and then cooling the product in liquid lead, it is possible to produce microscopic diamonds which can be separated from the iron mass by removing the metal with acids. The process is of no commercial value.

The electric furnace used to produce graphite on a commercial scale is a resistance furnace in which the solid charge itself acts as the resistance. The furnace is about 30 feet long by 2 feet square, and its permanent portion consists of a base and two upright ends through which several electrodes pass. The charge, which is about 6 tons in weight, consists of sand and anthracite ground to the size of rice. Since cold anthracite is a poor conductor of electricity, it is necessary to provide for preliminary heating; and this is accomplished by joining two of the electrodes by a thin carbon rod which runs through the centre of the charge and becomes heated by the passage of the current. The sides of the furnace are roughly built in with loose bricks before the run is commenced. 
The mechanism of the graphitization process is not fully understood. It is assumed that carbides are formed from the metallic constituents of the anthracite; that these carbides, formed in the hot zone, are almost immediately decomposed; that the carbon thus liberated is in the graphitic form; and that the volatile metals set free by the decomposition of the carbide again form carbides in another part of the furnace. In this way a comparatively small proportion of metal in the anthracite will suffice to convert the whole of the carbon present, viâ carbide, into graphite.

A run of the furnace described above occupies twenty hours; and the temperature attained appears to be above $2000^{\circ} \mathrm{C}$. The material is extracted by unbuilding the loose sides of the furnace after it has cooled.

The same type of furnace is employed to prepare graphite electrodes. A mixture of petroleum coke and pitch is moulded into the shape of the required electrodes, some carbide-forming material such as iron oxide or silica being added. After being baked, the rods are packed into the furnace transversely to the path of the current; and the graphitization is carried out as before.

The most important manufacturers of graphite are the International Acheson Graphite Co., whose works are at Niagara Falls; and to them is due the credit of having devised methods whereby graphite can be readily utilized as a lubricant. The Acheson Co. produce a very soft variety of graphite which, when reduced to an extremely fine state of division, is known as "Dag" (from the initials of Deflocculated Acheson Graphite). Mixed with grease, this comes on the market as Gredag; suspended in oil it is known as Oildag; whilst when water is substituted for oil the product is termed Aquadag. All three forms are excellent lubricants owing to their graphite content.

\section{Calcium Carbide.}

Calcium carbide is formed by the action of lime upon carbon in the electric furnace-

$$
\mathrm{CaO}+3 \mathrm{C}=\mathrm{CaC}_{2}+\mathrm{CO}
$$

Since under the conditions of the preparation the carbide is 
liquid, two possibilities present themselves : it is possible (1) to let the furnace contents solidify at the end of the run, or (2) to run off the liquid carbide from the furnace as it is formed. The furnaces used for the first process are termed ingot furnaces, whilst the second method is carried out by means of tapping furnaces.

One of the most effective types of ingot furnace is that devised by Willson. The actual furnace is a sheet-iron box about 6 feet high, mounted on wheels so that it can travel along a railway. The two graphite electrodes are mounted vertically over the line of rails and can be lowered into the box when it is in the proper position. After the contents of one box have been treated, the electrodes are raised; the box is rolled away; and another box is brought under the electrodes in its turn. In this way charges can be treated in succession by the same pair of electrodes; and no time is wasted in cooling the product. The furnace charge consists of burnt lime and anthracite or coke crushed to pieces about a quarter of an inch in diameter. Powdered material does not give such good results as granulated particles do. The charge is introduced down shoots and is kept on a level about 2 feet above the arc. It is stirred up at intervals to facilitate the escape of gases. The weight of the charge is about $10 \mathrm{cwt}$. and from this some $800 \mathrm{lb}$. of commercial carbide is obtained.

Other types of ingot furnace have no truck, but instead the bottom of the furnace opens outwards so as to allow the carbide to drop through. There is also the Horry type, in which the rotary principle is employed; so that once the carbide is formed it is removed into a cooler region, by the slow revolution of the furnace, whilst the arc plays upon fresh raw material.

Turning now to the tapping furnaces, these are furnished with a large vertical electrode, about 8 feet long, composed of rods held in a circular holder and having carbon paste rammed into the interstices of the mass. The furnace is permanent and is provided with a vent through which the molten carbide is drawn off (or "tapped") as it is formed.

The choice of the raw materials for calcium carbide manufacture requires care, as otherwise the process may be dangerous, or the resulting product may be contaminated with 
undesirable matter. The presence of magnesia interferes with the carbide formation; any phosphorus may give rise to phosphoretted hydrogen if the carbide is utilized for the production of acetylene; whilst if the coal used contains much mineral matter the end-product may be of bad quality.

The main commercial application of calcium carbide is the generation of acetylene; but in recent times, as will be seen in the following chapter, carbides have been employed in the fixation of nitrogen.

\section{Silicon and Silicon Carbide.}

The electric furnace is now employed for the manufacture of silicon on a commercial scale from silica and carbon. The exact details of the furnace vary according to the process used; but in general two carbon electrodes dip vertically into the charge. In some furnaces the two electrodes form the poles of the discharge; whilst in others the current passes between the vertical electrodes and the carbon lining of the furnace.

The reaction is simply one of reduction-

$$
\mathrm{SiO}_{2}+\mathrm{C}=\mathrm{Si}+\mathrm{CO}_{2}
$$

but in practice there is considerable difficulty in avoiding the formation of some silicon carbide as well-

$$
\mathrm{SiO}_{2}+3 \mathrm{C}=\mathrm{SiC}+2 \mathrm{CO}
$$

The charge consists of coke and silica in the form of sand or crushed flint. The main practical difficulty is found in regulating the temperature of the furnace; for if this be allowed to rise too high the silicon volatilizes and there is also some loss due to the formation of silicon carbide which contaminates the product. The heating effect is generally controlled within limits which allow the silicon, when formed, to melt and percolate down through the charge to the bottom of the furnace, whence it is run off.

Electric furnace silicon is a brittle crystalline body with a dark silver lustre. It contains about 2 per cent. of carbon along with lesser proportions of iron and aluminium. It can be cast into any form which may be required.

Commercially, silicon is employed in several ways. In 
steel-making, it is used in certain cases instead of ferro-silicon. It is also utilized as a substitute for aluminium in the thermit process for manufacturing low-carbon ferro-alloys; whilst a silicon-copper alloy, silicon-copper, plays a part as a deoxidizer in the making of copper castings.

More important than silicon itself is its carbide, carborundum, which is formed when carbon and silica are heated in the electric furnace under certain conditions. On a commercial scale, the furnace charge is made up of silicious sand, coke, sawdust, and about 2 per cent. of common salt. The sawdust is used in order to make the charge porous and thus permit the escape of the carbon monoxide formed in the reaction; whilst the addition of the salt is due to the fact that the furnace runs better when this flux is present.

The furnace employed resembles that used for graphite manufacture. It is about 16 feet long, 6 feet wide, and 5 feet high. Between the electrodes a core of coke is placed, running from end to end of the furnace. This core acts as a resistance and heats the surrounding charge by conduction. The run of the furnace lasts about thirty-six hours.

When the charge is broken up at the end of the run, it is found that the furnace contents are arranged around the core in a series of layers. Immediately surrounding the graphitized core lies a layer of crystallized carborundum about $1 \frac{1}{2}$ feet thick. This is the valuable part of the product. Outside this layer is found a greenish-coloured mass of impure amorphous silicon carbide which has no commercial value; whilst still further from the core the original materials of the charge are almost unaltered. These last two layers are usually worked up in the next charge.

Carborundum thus prepared is a dark brown or black crystalline substance. Owing to its extreme hardness, it is employed as an abrasive; its refractory nature makes it useful for lining steel and cement furnaces ; and it is also utilized as a deoxidizer in steel manufacture.

Silundum is a compact form of carborundum which is obtained by impregnating carbon with silicon vapour in the electric furnace. The charge may be composed of silica and carborundum or of sand and coke. In either case, the object is to produce silicon- 


$$
\begin{aligned}
& \mathrm{SiO}_{2}+2 \mathrm{SiC}=3 \mathrm{Si}+2 \mathrm{CO} \\
& \mathrm{SiO}_{2}+2 \mathrm{C}=\mathrm{Si}+2 \mathrm{CO}
\end{aligned}
$$

The carbon articles which it is desired to convert into silundum are packed in the charge separate from the furnace core. During the run, silicon vapour is formed which attacks the carbon of the models and converts them into silundum shapes. Silundum owes its technical value to the fact that it is not readily oxidized, which renders it extremely useful as a material for electrical resistances intended to work at high temperatures.

Two other silicon derivatives deserve mention here. The first of these is siloxicon, which is a mixture of various compounds of carbon, silicon, and oxygen, and has the general formula $\mathrm{Si}_{n} \mathrm{C}_{\mathrm{n}} \mathrm{O}$. It is formed in the electric furnace by using a carbon-silica charge in which the carbon is insufficient to form silicon carbide with the whole of the silicon present. Siloxicon is a useful refractory material. It is a greyish-green powder.

Monox is a mixture of silica, amorphous silicon, and silicon monoxide, SiO. Under electric furnace conditions, silicon monoxide is a gas; and if it be allowed to escape into an airfree chamber, a fine brown powder condenses. This is termed monox. The reaction appears to be as follows :-

$$
\mathrm{SiO}_{2}+\mathrm{C}=\mathrm{SiO}+\mathrm{CO}
$$

Monox is extremely light; a cubic foot of the powder weighs only $2 \frac{1}{2} \mathrm{lb}$. It was employed as a paint and also as an ingredient in printers' ink; but its manufacture has now been stopped.

\section{Silicides.}

Just as a carbon yields carbides of the alkaline earth metals, so silicon can form silicides of an analogous type-

$$
\begin{array}{cc}
\mathrm{CaC}_{2} & \mathrm{CaSi}_{2} \\
\text { Calcium carbide. } & \text { Calcium silicide. }
\end{array}
$$

These silicides are obtainable in either of two ways; for we may reduce the corresponding silicate with carbon in the 
electric furnace; or we may heat silica and carbon with the oxide or salt of the required alkaline earth metal-

$$
\begin{aligned}
\mathrm{CaH}_{2} \mathrm{Si}_{2} \mathrm{O}_{6}, \mathrm{H}_{2} \mathrm{O}+5 \mathrm{C} & =\mathrm{CaSi}_{2}+5 \mathrm{CO}+2 \mathrm{H}_{2} \mathrm{O} \\
\mathrm{CaO}+2 \mathrm{SiO}_{2}+5 \mathrm{C} & =\mathrm{CaSi}_{2}+5 \mathrm{CO}
\end{aligned}
$$

The formation of the silicides requires a temperature higher than that which suffices for carbide production.

The silicides are bluish-white in tint and of metallic appearance. Even at ordinary temperatures they oxidize slowly in air, yielding silica and the oxide of the metal from which they are derived. When treated with water, they liberate hydrogen-

$$
\mathrm{CaSi}_{2}+6 \mathrm{H}_{2} \mathrm{O}=\mathrm{Ca}(\mathrm{OH})_{2} \cdot 2 \mathrm{SiO}_{2}+5 \mathrm{H}_{2}
$$

This property makes them extremely useful for military purposes, such as inflating airships: for the plant required in the hydrogen generation is extremely simple; the efficiency of the silicides, weight for weight, is superior to that of metals and sulphuric acid; and the hydrogen thus produced is free from contamination by heavier gases.

Owing to this power of liberating hydrogen from water, the silicides are good reducing agents; and barium silicide has been employed on a commercial scale for converting indigo into indigo-white.

\section{Alundum.}

This material is an artificial corundum or emery. It is manufactured by first calcining bauxite in an ordinary furnace to remove combined water from the material; after which some carbon is added for the purpose of reducing any metallic impurities, such as titanium or iron, contained in the bauxite. The final stage in the process is fusion in the electric arc furnace. The charge is allowed to cool slowly ; crystallization takes place; and the metallic impurities segregate in nodules, which can be picked out after the charge has been crushed.

The crushed material is sorted out according to the size of the grains, and these are roasted in order to oxidize any metallic particles in them. After this the grains are mixed with fireclay, moulded into wheels, and fired in kilns. 


\section{Phosphorus.}

The electric furnace can be utilized to produce phosphorus either directly from bone-ash or from mineral phosphates. In either case, the reduction is carried out by mixing the phosphate with silica and charcoal, and subjecting the product to the action of an arc furnace with a vertical electrode. Phosphorus vapour, carbon monoxide, and a silicate slag are produced. The addition of the silica permits more rapid working ; as the slag can be run off in its molten form; thus time is saved in cleaning the furnace, which would have to be done if the by-products of the reaction were left to accumulate in the form in which they are produced.

\section{Carbon Disulphide.}

In the manufacture of carbon disulphide, the electric furnace appears likely to displace the older methods in a very brief time. The furnace actually employed is of a special pattern. Originally, horizontal carbon electrodes were used as terminals ; but these have now been dispensed with; and in the newest types of furnace the electrical connection is made directly with the carbon of the charge.

The actual furnace is a cylinder about 40 feet high and 16 feet in diameter. The walls of this tower are hollow and the sulphur is fed in near the top, forming a non-conducting layer within the walls and thus preventing loss of heat from the furnace. As it sinks down under the action of gravity, the sulphur melts and finally emerges on to the furnace hearth. The central hollow of the tower is filled with a closely-packed mass of carbon which is replenished from shoots at the side. The current is supplied, on the one hand, through the carbon in the shoots, and, on the other hand, through the furnace bed. On the hearth of the furnace the heat acts upon the mixture of carbon and sulphur, forming carbon disulphide and sulphur vapour. The latter, in its passage upwards, reacts with some of the carbon and yields more disulphide. The disulphide vapour finally passes out of the top of the tower and is condensed in suitable receptacles. The furnace is capable of running continuously for months at a time. 


\section{Alkali Manufacture.}

Hitherto in this chapter we have been concerned with electric furnaces in which the heating effect of the electric current played the main part in the process; but we must now turn to a different type wherein the action of the current is electrolytic in character. As an example of this, the manufacture of caustic soda and metallic sodium may be chosen.

In the Acker process for the commercial production of caustic soda and chlorine, fused sodium chloride is used as the electrolyte. Into this dips a series of carbon anodes; whilst the cathode is a mass of molten lead. The whole arrangement is gas-tight; there is an outlet for chlorine above the anodes and an injector through which steam can be forced. The inlet and outlet are separated from one another by a trap.

The reactions which take place during the operation of the furnace are as follows. The sodium chloride is electrolysed, giving gaseous chlorine (which passes off through the outlet) and sodium (which forms an alloy with the molten lead of the cathode). This molten material then circulates through a trap into another compartment where it meets a blast of steam which attacks the sodium and produces caustic soda solution, as well as hydrogen. The hydrogen is emitted through a flue; the alkaline liquid floats on the top of the molten lead and is drawn off through an outlet at the proper level; whilst the lead passes again into the electrolytic chamber to take its place in the cathode mass. The hydrogen is utilized in burners for the preliminary fusion of the charge ; the chlorine is employed for the manufacture of bleaching powder. It will be seen that the successful operation of the method depends almost entirely upon the proper circulation of the molten lead; for the sodium alloy decomposes if left long in contact with the fused sodium chloride.

Turning now to the problem of manufacturing metallic sodium, the Castner process is the most successful on a large scale. The raw material used in it is caustic soda which is electrolysed in the fused condition. Apparently the following series of changes takes place during the electrolysis. In the first place, the fused caustic soda is electrolysed-

$$
2 \mathrm{NaOH}=2 \mathrm{Na} \text { (at cathode) }+2 \mathrm{OH} \text { (at anode) }
$$


At the anode, the hydroxyl ions combine to form water and oxygen-

$$
4 \mathrm{OH}=2 \mathrm{H}_{2} \mathrm{O}+\mathrm{O}_{2}
$$

This water may then be supposed to become electrolysed, yielding hydrogen at the cathode and oxygen at the anode-

$$
2 \mathrm{H}_{2} \mathrm{O}=2 \mathrm{H}_{2} \text { (at cathode) }+\mathrm{O}_{2} \text { (at anode) }
$$

Collecting these changes together in one equation we have-

$$
2 \mathrm{NaOH}=\underset{\text { (at cathode) (at anode) }}{\mathrm{Na}_{2}+\mathrm{H}_{2}+\mathrm{O}_{2}}
$$

A technical difficulty appears at this point. If we place the electrodes far apart from one another, we shall incur a considerable expense in current. On the other hand, if we place them close together, we run the risk of the sodium being attacked by the liberated oxygen with disastrous results.

This danger has been obviated in an ingenious manner. The furnace is designed in the form of a cast-iron crucible with a furnace underneath for fusing the caustic soda at the beginning of the process. Through the bottom of the crucible, the cathode protrudes vertically. Surrounding this in the upper part of the crucible is an annular nickel anode, the distance between the two being only an inch in some types of furnace. Between cathode and anode lies a cylinder of nickel gauze which extends upward above the top of the cathode and ends in a metal cylinder. This gauze screen guides the sodium as it floats upward from the cathode through the fused electrolyte; and at the same time it prevents the stream of oxygen from the anode from coming in contact with the newly-formed metal. It is found that the process works in a satisfactory manner.

The Ashcroft sodium process depends upon a different series of reactions. The plant is in two parts connected by a pipe. In the first tank, fused sodium chloride is electrolysed, a molten lead cathode being employed as in the Acker process. This molten alloy is then circulated through the pipe to the second tank, which contains fused caustic soda. In this second tank there are two electrodes. Sodium is liberated at the cathode and hydroxyl ions at the anode. These hydroxyl ions then attack the sodium in the lead alloy, yielding caustic soda. In 
this way the loss of sodium from the fused caustic soda is continually made good. The liberated sodium floats to the surface of the fused electrolyte and is removed by a cowl and tube.

It is claimed that the Ashcroft process, when worked on a large scale, will be more efficient than the Castner process for three reasons. In the first place, it uses as a raw material common salt, which is cheaper than caustic alkali; secondly, chlorine is a valuable by-product of the process; and, thirdly, the current consumption in the electrolysis of the fused alkali corresponds to a production of sodium only; whilst in the Castner process half the current is employed in producing hydrogen.

Enough has now been said to give the reader some idea of the electroly tic furnace; and it is unnecessary to deal with other types, as this chapter is not intended to contain a complete account of the electric furnace industry. Further information on the subject must be sought in works of reference. ${ }^{1}$ It will suffice to mention the application of the electric furnace to metallurgical problems and to the glass and fused quartz industry; whilst its use in the fixation of nitrogen will be described in the next chapter.

1 See among others: Stansfield, The Electric Furnace; Wright, Electric Furnaces and their Industrial Applications; Martin, Industrial Inorganic Chemistry ; Rideal, Electrometallurgy; Pring, The Electric Arc. 


\section{CHAPTER II}

THE FIXATION OF NITROGEN

\section{The Circulation of Nitrogen in Nature.}

ANimals have two fundamental needs: oxygen and nourishment. Oxygen they can derive direct from the air, but nourishment can only be obtained by a more roundabout process. At the basis of all animal structure lies a group of substances called proteins, which the animal, being unable to synthesize itself, obtains from vegetables. These proteins contain from 15 to 20 per cent. of nitrogen. It has been shown that non-nitrogenous vegetable products, such as sugars, are incapable of supporting life. Animals, therefore, subsist upon the nitrogenous bodies of the vegetable kingdom; but this only carries us a step further back, and leaves us to discover how this nitrogenous matter is obtained by plants. It is found that part of the nitrogen in plants is derived from the air by means of certain bacteria, but the major part of it comes from the decomposition of plant tissue by one means or another. For example, a plant may be eaten by an animal; a certain amount of the nitrogen is excreted in the form of urea derivatives and the like; these break down to ammonia, which is then oxidized by bacteria to nitrous and nitric acids; and these, in turn, are absorbed by the vegetable world once more.

If one could enclose a few plants and a suitable series of animals in a bell-jar, this process of nitrogen circulation might conceivably be prolonged indefinitely; but, unfortunately, when we have to deal with the matter from a practical standpoint, the question of waste bulks very largely. The scheme below shows the main steps in the process of circulation, and we have now to consider at what points waste is to be expected. 
Atmospheric NitrogeN
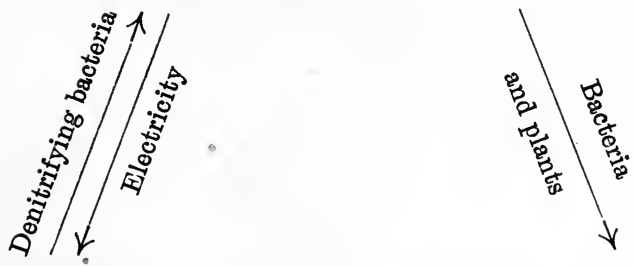

NitRIC ACID $\longrightarrow$ Plant life $\longrightarrow$ ORGANIC NITROGEN COMPOUNDS

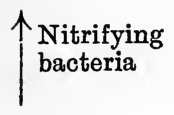

NITROUS ACID $\leftarrow$ Nitrifying bacteria- $\downarrow \begin{aligned} & \text { Plant decay } \\ & \text { Animal digestion } \\ & \text { Distillation }\end{aligned}$

Ammonia

In the first place, we must bear in mind that though the vegetable kingdom as a whole has its uses in the question of respiration, where it counterbalances the loss of oxygen due to the animal kingdom, it is only certain groups of plants which can serve as food. Thus any nitrogen which is absorbed by non-edible plants is, temporarily at least, lost from the point of view of nourishment. Again, we dispose of the greater part of our sewage by running it into the sea; but in this way the larger portion of the nitrogen contained in it is lost to us and goes to build up sea-plants, for which we have at present no use. Further, the nitrous and nitric acids produced from ammonia in nature do not necessarily remain on the spot where they are produced; they may disperse and be lost, as far as practical purposes are concerned.

In the past, these losses have been counterbalanced by two methods : either by inoculating plants with nitrifying bacteria, or by manuring the soil in which they grow. With the former method we have no concern here, but we must enter into some consideration of the second.

At the present day, the nitre beds ${ }^{1}$ of Chile and Peru supply the greater part of the cheap nitrogenous matter which is required in agriculture; and the extent of the demand can be gauged from the following figures which give the amounts exported in various years :-

1 See Norton, Consular Report on the Utilization of Atmospheric Nitrogen, Washington, 1912; Martin, Industrial Inorganic Chemistry, I., 432. 


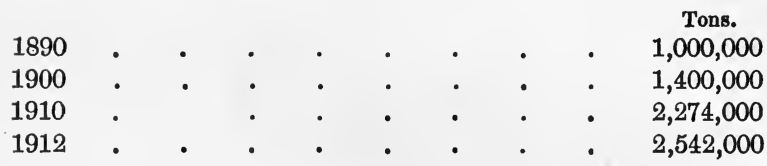

Within quite recent times, fresh nitre beds have been discovered in South America, so that there is no immediate prospect of exhaustion in that quarter. None the less there is a possibility of shortage, and within a measurable time we shall be faced with the problem of producing nitrogenous substances upon a vast scale and at a reasonably low cost. ${ }^{1}$ By adopting a less wasteful method of sewage disposal, and perhaps by utilizing as nitrogen sources such substances as the seaweed from the Sargasso Sea, we may hope to diminish the amount of synthetic material required; but at the best we cannot hope to dispense with it altogether.

During the war, the Germans were cut off from the natural sources of nitrates; and in order to keep up their supply of munitions they were driven to utilize synthetic methods: so that it seems probable that a very large amount of suitable plant is now in existence. ${ }^{2}$

For synthetic purposes, the cheapest source of nitrogen is the atmosphere; and various methods are now available, by means of which atmospheric nitrogen can be converted into compounds suitable for manures. This process has been termed the fixation of nitrogen; and in the following sections of this chapter we shall deal with the main methods which have been devised with that end in view.

\section{The Production of Nitrogen Oxides, Nitrous and Nitric Acids.}

In any technical process we must bear in mind two things which govern the price of the final product: the cost of the starting materials, and the expenditure of energy necessary to convert them into the compound required. Now, in the manufacture of nitrogen compounds, the cheapest source of nitrogen

1 See Crookes, The Wheat Problem, for an account of the food question; and for the chemical side, Knox, The Fixation of Atmospheric Nitrogen, and Martin, Industrial Inorganic Chemistry, Vol. I.

2 For the British side, see Dobbie, Trans., 1920, 117, 430. 
is certainly the atmosphere: but we have not only to obtain nitrogen; we have also to find some comparatively cheap substance with which to combine it; and, finally, we must devise an economical method of effecting the combination.

It is obvious that oxygen is the cheapest element we could choose in order to form a compound with nitrogen, for the two occur together in the atmosphere in what is, for practical purposes, an unlimited quantity. The question then arises: Can we convert nitrogen into its oxides with an expenditure of energy low enough to make it pay in practice? Up to the present time, the methods applied to this question have been almost entirely electrical ; so that the problem, in this department at least, rests chiefly upon the cost of electricity. The electric power, in most processes, is utilized in the form of an arc.

Crookes, ${ }^{1}$ in 1892 , showed that, at the temperature of a high tension arc, oxygen and nitrogen combined to form nitric oxide. Rayleigh ${ }^{2}$ five years later proved that in this way 25 c.c. of oxides of nitrogen could be produced by the expenditure of one watt-hour. The action of the arc appears to be due both to its heating effect and to electrical influences. ${ }^{3}$

At this point it may be well to recall the work of Nernst ${ }^{4}$ upon gas equilibria at high temperatures. If we take the case of the union of nitrogen and oxygen to form nitric oxide, we have the following equation:-

$$
\mathrm{N}_{2}+\mathrm{O}_{2} \rightleftharpoons 2 \mathrm{NO}
$$

An examination of this equation will show that the volume of gas at the beginning of the reaction is the same as that at the end. Now, if no change in volume takes place during a reaction, it is clear that the position of equilibrium of such a reaction would be uninfluenced by the pressure under which it is carried out.

1 Crookes, Chem. News, 1892, 65, 301.

2 Rayleigh, Trans., 1897, 71, 181.

3 McDougall and Howles, Mem. Manchester Phil. Soc., 1900, 4, 44 ; Muthmann and Hofer, Ber., 1903, 36, 438; .Le Blanc and Nüranen, Zeitsch. Electrochem., 1907, 13, 297 ; Gran and Russ, ibid., 345, 573 ; Lee and Beyer, ibid., 701 ; Foerster, ibid., 1906, 12, 536 ; compare Haber and Koenig, ibid., 1907, 13, 725; 1908, 14, 689; Warburg, ibid., 1906, 12, 540. A full summary of the evidence is to be found in Knox, The Fixation of Atmospheric Nitrogen.

4 Nernst, Application of Thermodynamics to Chemistry, 1907. 
It is obvious that the position of equilibrium in a reversible reaction may be very largely dependent upon the temperature at which the reaction is carried out. Nernst has shown, for instance, that if air be passed through a tube heated to $1538^{\circ} \mathrm{C}$., only 0.4 per cent. of nitric oxide is formed; while at $1922^{\circ} \mathrm{C}$. the quantity rises to 1 per cent. The results appear to be in contradiction to the fact that nitric oxide decomposes at a white heat. The matter will be made clear if we take into account the velocities with which the two reactions-

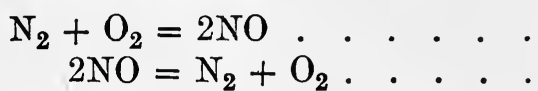

proceed. According to the Guldberg-Waage Law of Mass Action, the amount of chemical action is proportional to the active masses of the substances reacting. Thus the chemical action corresponding to equation (1) will be proportional to the amounts of nitrogen and oxygen present; while equation (2) represents an action which will be proportional to the amount of nitric oxide used. Thus if $v_{1}$ be the velocity of formation of nitric oxide, then we have the equation-

$$
v_{1}=k_{1} \times\left(\mathrm{N}_{2}\right) \times\left(\mathrm{O}_{2}\right)
$$

where $\left(\mathrm{N}_{2}\right)$ and $\left(\mathrm{O}_{2}\right)$ are the active masses of nitrogen and oxygen present. Again, if $v_{2}$ be the velocity of formation of nitrogen and oxygen from nitric oxide, then-

$$
v_{2}=k_{2} \times(\mathrm{NO}) \times(\mathrm{NO})=k_{2} \times(\mathrm{NO})^{2}
$$

Suppose, now, that the two reactions have proceeded until equilibrium has been reached. At this point, the amount of nitric oxide formed is equal to that decomposed. Consequently-

$$
v_{1}=v_{2}
$$

and the equilibrium constant, $K$, is the ratio of the one velocity constant to the other. In other words-

$$
\mathrm{K}=\frac{k_{2}}{k_{1}}
$$

Now, the values of $k_{1}$ and $k_{2}$ can be ascertained experimentally, so that we can easily determine the value of the equilibrium constant $\mathrm{K}$. 
When we apply this line of argument to the concrete case of the production of nitric oxide from the air, we proceed as follows. It is evident that the equilibrium constant $K$ will be dependent upon the active masses of the three gases present, nitrogen, oxygen, and nitric oxide, and will be expressed thus-

$$
\mathrm{K}=\frac{(\mathrm{NO})^{2}}{\left(\mathrm{~N}_{2}\right) \times\left(\mathrm{O}_{2}\right)}
$$

Let $x$ be the amount of nitric oxide produced at the equilibrium point; then, since the concentration of nitrogen at the start is 79 per cent., and that of oxygen is 21 per cent. (these being the proportions in which these gases occur in air), we have at constant temperature the following equation :-

$$
\mathrm{K}=\frac{x^{2}}{\left(79-\frac{x}{2}\right)\left(21-\frac{x}{2}\right)}
$$

Since we can determine $K$ experimentally, we can solve the equation for $x$, and this will give us the quantity of nitric oxide formed when the equilibrium point is reached.

Now, $K$ alters with the temperature according to the equation-

$$
\frac{d \log _{\epsilon} \mathrm{K}}{d \mathrm{~T}}=-\frac{\mathrm{Q}}{2 \mathrm{~T}^{2}}
$$

where $\mathrm{Q}$ is the change of energy in the system, and $\mathrm{T}$ the absolute temperature. Integrating this under the assumption that $Q$ is independent of the temperature (as it apparently is for all practical purposes in the case we are considering), we then have for two temperatures $\mathrm{T}_{0}$ and $\mathrm{T}_{1}$ the equation-

$$
\log _{\epsilon} \mathrm{K}_{1}-\log _{\epsilon} \mathrm{K}_{0}=\frac{\mathrm{Q}}{2}\left(\frac{1}{\mathrm{~T}_{1}}-\frac{1}{\mathrm{~T}_{0}}\right)
$$

Thus, if we know the equilibrium constant $\mathrm{K}_{0}$ for temperature $\mathrm{T}_{0}$, and also the energy change in the system, we can calculate from this last equation what the equilibrium constant will be for any other temperature $T_{1}$. The energy change in the system can be determined by finding the equilibrium constant for two temperatures, and then substituting in the above equation. In this way, from a couple of measurements at different temperatures, we shall be in a position to foretell 
the equilibrium constant of the reactions for any temperature. The agreement between theory and experiment is quite close, as the following figures ${ }^{1}$ show :-

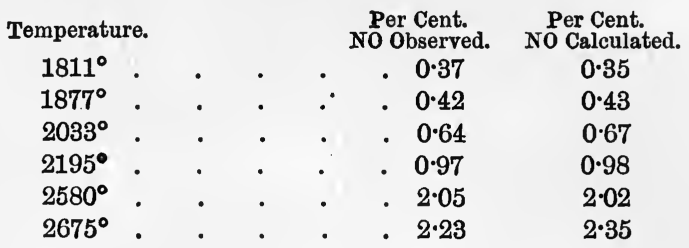

Another point of importance arises in this connection. From what has already been said, it is clear that the velocity of decomposition of nitric oxide is very greatly influenced by the temperature at which the reaction is carried out. If we can bring the reactions into equilibrium at a temperature of about $2500^{\circ} \mathrm{C}$., we shall have in the mixture, according to Nernst's data, about. 2 per cent. of nitric oxide. If we cool the gases down slowly, they will come into a fresh state of equilibrium for each temperature they pass through, and consequently when we reach ordinary temperatures the percentage of nitric oxide will be negligible. On the other hand, if we produce a sudden drop in the temperature, the gases will not attain equilibrium quickly enough to keep pace with the fall in temperature, and consequently we shall be able to reach ordinary temperatures before the nitric oxide has time to decompose. Once the ordinary temperature is attained, the decomposition of nitric oxide will proceed with such slowness as to be negligible from the practical point of view. Thus, if we could cool the mixture of gases instantaneously from $2500^{\circ} \mathrm{C}$. to zero, we should preserve the 2 per cent. of nitric oxide which it contains at the higher temperature. Of course, in practice such instantaneous cooling is impossible; but if the gases be removed rapidly from the neighbourhood of the source of heat, it is possible to stave off the decomposition of the nitric oxide to a certain extent.

In the McDougall and Howles process, the mixture of oxygen and nitrogen is passed, over a stationary electric arc, and the cooling is achieved by using a rapid stream of gas, so that the oxides are swept along in the current and carried

1 Nernst, Application of Thermodynamics to Chemistry, p. 35. 
away from the arc as quickly as possible. An improvement ${ }^{1}$ upon this was devised by Birkeland and Eyde, whose process has been utilized on a commercial scale at Notodden, in Norway. In this process advantage is taken of the effect which a strong electro-magnet exerts upon arcs. If we fix opposite the middle of an electric arc a strong electro-magnet, the arc will be diverted and finally broken; after which it will reform between the electrodes, be diverted and broken again. By this means we replace the simple arc by a series of arcs, which form, bend outwards, and break. If the apparatus be properly adjusted, it is possible by this means to produce an are in the form of a thin disc instead of the usual narrow stream. The advantages of the Birkeland and Eyde method are twofold: in the first place, since the disc of the arc is thin, a gas passing through it is only heated for a very brief interval; secondly, owing to the increase in area of the arc, it is possible to pass a much larger quantity of gas through the hot zone than is the case when the gases are passed across an ordinary arc. After the gases have been passed through the arc they are conducted into a cooling apparatus; they are next led into an oxidizing chamber, where the nitric oxide becomes nitric peroxide. The final product of the process is basic calcium nitrate; the basic salt is prepared in preference to the normal nitrate, owing to the fact that the latter is too deliquescent for commercial purposes.

In the Pauling process ${ }^{2}$ an analogous type of arc is produced without the use of an electro-magnet in the following way. The two electrodes are composed of metal tubing and are cooled by an internal stream of water. They are bent into a rough V-shape and placed so that the points of the V's are only about $4 \mathrm{~cm}$. apart, thus : $><$. At the point where they are nearest, two thin iron blades jut out which approach within a couple of millimetres of one another; and the arc strikes here. Below the V's, a strong air-blast is situated. The action of the apparatus is as follows. The arc strikes between the iron points; the air-blast then blows the arc in front of it and the arc runs along the arms of the $\mathrm{V}$ opposite to the jet-nozzle, growing in

1 Birkeland, German Patent, No, 179882.

2 See Scott, Jour. Roy. Soc. Arts, 1912,60,645 ; also Zeitsch. Elektrochem., $1907,13,225$; 1909, 15, 544; 1911, 17, 431. 
area as it runs, since the points on the electrodes between which it plays are further and further apart as it flows up the arms of the V's. Finally, the distance becomes too great for the current to bridge; the arc breaks ; and a new arc is reformed at the iron points.

Another modification is due to Schönherr. ${ }^{1}$ In his process the arc is stationary and several metres in length. It is surrounded by a tube through which the air is driven in a spiral current, the spiral being produced by using a rotating jet to introduce the air current into the tube. In this way the mixture of oxygen and nitrogen passes alternately across the arc and into the cooler region on either side, so that it is subjected to a rapid succession of hot and cold periods. This process is said to be the most economical of its kind.

\section{The Manufacture of Ammonia.}

In the previous section we dealt with the processes suggested for the union of nitrogen with oxygen, and it was pointed out that the use of oxygen was dictated by its comparative cheapness; in the present section the union of nitrogen with hydrogen will be considered, as, next to oxygen, hydrogen is the least costly element-at least, when it can be utilized in the form of producer gas or some other such mixture.

The work of Regnault ${ }^{2}$ on the spark discharge, and that of Donkin ${ }^{3}$ upon the silent discharge, had shown that these two forms of electric energy were capable of causing the direct union of nitrogen with hydrogen. It was not till about ten years ago, however, that this process was brought into effective touch with the technical side of the problem. In 1900, de Hemptinne, ${ }^{4}$ using both the types of discharge, made an investigation of the proper conditions under which the action should be carried out. It had been proved by Ramsay and Young ${ }^{5}$ that ammonia begins to decompose at a temperature of $500^{\circ} \mathrm{C}$., and is completely broken up at a little below $800^{\circ} \mathrm{C}$.; so that it was to be expected that the best results would be

1 Elektrotechnische Zeitschrift, 1909, 30, 365, 397.

2 Regnault, Traite de chemie, 1846.

3 Donkin, Proc. Roy. Soc., 1873, 21, 281.

${ }_{4}$ De Hemptinne, Bull. Acad. roy. Belg., 1902, 28.

5 Ramsay and Young, Trans., 1884, 45, 88. 
obtained by synthesis at comparatively low temperatures. This view is confirmed by de Hemptinne's results. He found that the yield was greatest when three conditions were observed : first, the temperature must be kept down to the point at which ammonia liquefies; second, the pressure under which the experiment is carried out must be low; and lastly, the gap across which the discharge acts must be narrow. Schlutius ${ }^{1}$ has patented a somewhat analogous process in which, instead of a mixture of hydrogen and nitrogen, he employs "Dowson gass," which is obtained by passing a mixture of air and steam over glowing coal. The resulting gas has approximately the following composition :-

Per Cent.

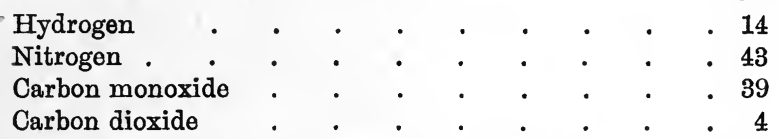

This mixture is acted upon by the silent electric discharge in presence of moist platinum, and the nature of the resulting compound depends upon the temperature at which the reaction is carried out. If the apparatus be kept below $80^{\circ} \mathrm{C}$., ammonia is formed in accordance with the equation-

$$
\mathrm{N}_{2}+3 \mathrm{H}_{2}+2 \mathrm{H}_{2} \mathrm{O}=2 \mathrm{NH}_{3} \text {, aq }
$$

On the other hand, if we allow the temperature to exceed $80^{\circ} \mathrm{C}$., the carbon monoxide also reacts, with the formation of ammonium formate-

$$
\mathrm{N}_{2}+3 \mathrm{H}_{2}+2 \mathrm{CO}+2 \mathrm{H}_{2} \mathrm{O}=2 \mathrm{H} . \mathrm{COONH}_{4}
$$

We must now turn to the consideration of the action of catalytic agents upon the union of nitrogen with hydrogen. De Lambilly ${ }^{2}$ has investigated this problem, and has taken out a patent for a process based upon his results. He was struck by the fact that the heats of formation of ammonia and ammonium hydrate are less than those of ammonium carbonate and ammonium formate. From this it follows that the expenditure of energy required to produce the salts is less than is necessary in the case of the free ammonia or ammonium

1 Schlutius, English Patent, No. 2200 (1903).

2 De Lambilly, German Patent, No. 74274. 
hydrate. De Lambilly therefore devoted himself to the production of the salts rather than the free substance. His process is as follows. Air and steam are led over white-hot coke, and from the mixture of gases thus produced, either carbon dioxide or carbon monoxide is removed, according as ammonium formate or bicarbonate is required. The gases are then passed through tubes filled with porous substances, such as platinized pumice, wood-charcoal, bone-charcoal, or spongy platinum. After they have passed once through the tubes, the gases are mixed with steam and again sent through the apparatus. The reactions which take place depend upon whether carbon monoxide or carbon dioxide be used-

$$
\begin{aligned}
& \mathrm{N}_{2}+3 \mathrm{H}_{2}+2 \mathrm{CO}_{2}+2 \mathrm{H}_{2} \mathrm{O}=2 \mathrm{HO} . \mathrm{CO}^{\circ} \mathrm{ONH}_{4} \\
& \mathrm{~N}_{2}+3 \mathrm{H}_{2}+2 \mathrm{CO}+2 \mathrm{H}_{2} \mathrm{O}=2 \mathrm{H} . \mathrm{CO} . \mathrm{ONH}_{4} .
\end{aligned}
$$

In the case of equation (I.), it is found that the most favourable temperature lies between $40^{\circ}$ and $60^{\circ}$, while most ammonium formate (II.) is produced between $80^{\circ}$ and $130^{\circ}$.

The main fault of the process thus outlined lies in the tendency of the ammonium formate to decompose into water and hydrocyanic acid. In order to evade this difficulty, De Lambilly ${ }^{1}$ utilized the same apparatus, but passed through it a mixture of carbon monoxide and ammonia, the latter being obtained from ammonium hydrate. In this case the best temperature seems to lie between $150^{\circ} \mathrm{C}$. and $180^{\circ} \mathrm{C}$.

By far the most important of the modern synthetic methods of preparing ammonia is due to Haber and Le Rossignol. $^{2}$ An examination of the equation for the reaction-

$$
\mathrm{N}_{2}+3 \mathrm{H}_{2} \rightleftharpoons 2 \mathrm{NH}_{3}
$$

shows that four volumes of nitrogen and hydrogen give rise to two volumes of ammonia; whence it is clear that high pressures will favour the conversion. A further point arises when we consider that the formation of ammonia in this way is an exothermic reaction; for this indicates that the lowest

1 De Lambilly, German Patent, No. 78573.

2 See Haber and van Oordt, Zeitsch. anorgan. Chem., 1905, 43, 111; 44, 341 ; Haber and Le Rossignol, Zeitsch. Elektrochem., 1908, 14, 181, 513 ; ibid., 1913, 19, 53; Haber, The Thermodynamics of Technical Gas Reactions; Bernsthen, Eighth Internat. Congress of Applied Chemistry, 1912 (Abstracted in J. Soc. Chem. Ind., 1912, 31, 982). 
possible temperatures should be employed. But if low temperatures are used, then the velocity of combination of the nitrogen and hydrogen will be reduced; so it is evident that the reaction must be hastened by the use of catalytic agents. In other words, the conditions of success are: (1) high pressure ; (2) low temperature; and (3) an efficient catalyst.

On the technical scale, the outline of the process is as follows. Nitrogen and hydrogen, in the proportions of one volume to three, and under a pressure of 200 atmospheres, are electrically heated to between $800^{\circ} \mathrm{C}$. and $1000^{\circ} \mathrm{C}$. The mixed gases then pass over a layer of the catalyst, which may be iron or uranium.* Ammonia is thus formed; and the product is removed by a liquefaction process. The unchanged nitrogen and hydrogen are then mixed with fresh supplies and are returned through the apparatus; so that the process is a continuous one.

The Serpek process ${ }^{1}$ is noteworthy in that along with ammonia it yields a valuable by-product. The outline of the method is as follows. A mixture of powdered bauxite and carbon is introduced into a rotating, inclined kiln; and during its passage it is calcined by the action of heated gases derived from another stage of the process. The calcined bauxite is ejected from the kiln into a hopper, wherein it is mixed with the calculated quantity of carbon; and from this hopper the mixture sinks into an inclined cylinder, one part of which is electrically heated to $1800^{\circ} \mathrm{C}$. As the charge slides down the cylinder it is met and traversed by a stream of gas from a generator, the composition of the gas being about 30 per cent. carbon monoxide and 70 per cent. nitrogen. Aluminium nitride is collected at the lower end of the cylinder. The impurities due to the bauxite appear to be oxidized and volatilized during the process; for the end-product is remarkably pure. The surplus gas is conducted upwards and serves to calcine the fresh bauxite.

The reaction which takes place in the electrically heated zone appears to be this-

$$
\mathrm{Al}_{2} \mathrm{O}_{3}+3 \mathrm{C}+\mathrm{N}_{2}=2 \mathrm{AlN}+3 \mathrm{CO}
$$

* Osmium is a better catalyst, but ís too rare for use on a commercial scale.

1 For a fuller account of the Serpek process, see Knox, Fixation of Atmospheric Nitrogen. 
The aluminium nitride so produced can be broken up by acids or bases with the formation of ammonia and alumina; and as the latter is pure, it is very suitable for conversion into aluminium-

$$
\mathrm{AlN}+3 \mathrm{H}_{2} \mathrm{O}=\mathrm{Al}(\mathrm{OH})_{3}+\mathrm{NH}_{3}
$$

Temperature appears to be a weighty factor in the process; and impurities also play a part as catalytic agents.

The last method with which we need deal was originated by Kaiser. ${ }^{1}$ Calcium, when heated to redness in a stream of hydrogen, is converted into calcium hydride; and if this body is heated and nitrogen passed over it, calcium nitride is produced. When this in turn is heated in a stream of hydrogen, ammonia is liberated. The process is expressed in the equations below-

$$
\begin{aligned}
\text { (I.) } \mathrm{Ca}+\mathrm{H}_{2} & =\mathrm{CaH}_{2} \\
\text { (II.) } 3 \mathrm{CaH}_{2}+2 \mathrm{~N}_{2} & =\mathrm{Ca}_{3} \mathrm{~N}_{2}+2 \mathrm{NH}_{3} \\
\text { (III.) } \mathrm{Ca}_{3} \mathrm{~N}_{2}+6 \mathrm{H}_{2} & =\mathrm{CaH}_{2}+2 \mathrm{NH}_{3}
\end{aligned}
$$

It appears to be immaterial whether the gases are allowed to enter the tube together or separately, the action seems to go on with the same ease in either case.

\section{The Synthesis of Cyanides.}

In the foregoing sections we have dealt with the preparation of ammonia and of nitrates, both of which are naturally occurring substances; the present section deals with another set of compounds which at first sight appear most unpromising from the agricultural point of view, owing to their poisonous character. In actual practice, however, the cyanides appear to offer many advantages when used as manures.

The technical application of cyanides had led to the devising of methods for the production of these substances long before the question of nitrogen fixation became acute. The South African gold industry demanded a heavy output of cyanide for use in the cyanide process of extraction, and consequently a very considerable number of processes have come into existence for the purpose of supplying this need. With these we

1 Kaiser, French Patent, No. 350966; compare Haber and Oordt, Zeitsch. anorgan. Chem., 1905, 43, $111 ; 44,341$. 
have no concern here, but will confine ourselves to the chief methods which have been suggested within the last ten years or so.

Next to oxygen and hydrogen, carbon is probably the cheapest element which we can employ to form compounds of nitrogen. Whether it be utilized in the form of coal or coke, it is comparatively cheap and easily procurable, so that it is not surprising that attempts have been made to employ it in the fixation of nitrogen. Before dealing with the later processes which depend directly upon coal, however, we must glance at one or two others which are based upon other substances.

It was long ago shown by Berthelot that if a stream of mixed nitrogen and acetylene was raised to a high temperature, hydrocyanic acid was formed-

$$
\mathrm{C}_{2} \mathrm{H}_{2}+\mathrm{N}_{2}=2 \mathrm{HCN}
$$

Hoyermann ${ }^{1}$ proposed to utilize this reaction in technical practice by passing a mixture of one part of acetylene and two parts of nitrogen over an electric arc. In this way 60 to 70 per cent. of the acetylene was converted into hydrocyanic acid. The chief drawback of the method lies in the heavy deposits of carbon which take place on the electrodes. O'Neill ${ }^{2}$ employed a mixture of air with petroleum vapour or coalgas, instead of acetylene and nitrogen. Gruszkiewicz ${ }^{3}$ utilized "Dowson gas," which he passed over a flaming electrode. The best results were obtained when the percentage of carbon monoxide in the Dowson gas was raised to about 50 .

We must now turn to another set of methods, in which, instead of acetylene, metallic carbides are used. The work of Desfosses, ${ }^{4}$ Fownes, ${ }^{5}$ Bunsen and Playfair ${ }^{6}$ showed that when carbon was heated with non-volatile alkalis, the resulting products contained cyanogen compounds from which ammonia could be obtained. Attempts were thereafter made by various workers to utilize the reaction on a large scale, using barium

1 Hoyermann, Chem. Zeit., 1902, 26, 70.

2 O'Neill, Electrical World, 1902, 40, 1009.

${ }^{3}$ Gruszkiewicz, Zeitsch. Electrochem., 1903, 83.

${ }^{4}$ Desfosses, J. Chim. Pharm., 1828, 14, 280.

5 Fownes, J. pr. Chem., 1842, 26, 412.

6 Bunsen and Playfair, J. pr. Chem., 1847, 42, 397. 
hydrate as the alkali, but none of them seem to have come to much, owing to the effect which the high temperatures required had upon the apparatus.

The invention of the electric furnace, of course, gave a great impetus to technical research in this department, and as a result most of the difficulties experienced by the older workers have been evaded in modern practice.

The Ampère Electrochemical Company ${ }^{1}$ employ the following method. The starting materials are coke and a mixture of barium hydrate and carbonate. The quantities are so chosen as to provide an excess of coke over and above the amount required for the formation of barium carbide; the reason for this is that unless excess of coke is present, the mixture does not possess sufficient porosity to allow the passage of gases through it. The mixture is placed in a revolving electric furnace and the current is switched on. The action which takes place in the heated portion of the furnace is the formation of barium carbide, which fuses and flows over the unaffected part of the coke. The revolution of the furnace now carries this into a colder zone, in which it cools down and solidifies. At this point in the revolution it encounters a stream of nitrogen, which converts the carbide into the cyanide-

$$
\mathrm{BaC}_{2}+\mathrm{N}_{2}=\mathrm{Ba}(\mathrm{CN})_{2}
$$

The further revolution of the furnace brings the formed cyanide into a cooler section, where it is withdrawn from the apparatus. The emptied part of the machine then passes another point, where it is refilled with the mixture of coke and barium salts, and the process continues as before.

In this method ${ }^{2}$ there is formed as a by-product the substance barium cyanamide-

$$
\mathrm{BaC}_{2}+\mathrm{N}_{2}=\mathrm{BaN} . \mathrm{CN}+\mathrm{C}
$$

This substance is a deriative of cyanamide, which in turn is an ammonia substitution product-

$$
\begin{array}{ccc}
\mathrm{Ba}: \mathrm{N} . \mathrm{C} \equiv \mathrm{N} & \mathrm{H}_{2} \mathrm{~N} . \mathrm{C} \equiv \mathrm{N} & \mathrm{C}_{3} \mathrm{~N} \\
\text { Barium cyanamide. } & \text { Ammoniale. } & \text { Ammonia. }
\end{array}
$$

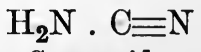

1 Dingler's Polytech. Jour., 1903, 33, 524.

2 French, Bericht d. V. Intern. Kongr. f. Angew. Chem., 1905, III., 727 ; Caro, Zeitsch. angew. Chem., 1906, 19, 1569. See also Zeitsch. angew. Chem., $1903,16,520$. 
On repeating the experiment with calcium salts instead of barium ones, it was found that the by-product of the one reaction became the main product of the second; so that the process yielded chiefly calcium cyanamide. The substance, which is technically termed "nitrolim," when heated with water under pressure liberates ammonia, as shown in the following equation :-

$$
\mathrm{Ca}: \mathrm{N} \cdot \mathrm{C} \equiv \mathrm{N}+3 \mathrm{H}_{2} \mathrm{O}=\mathrm{CaCO}_{3}+2 \mathrm{NH}_{3}
$$

The same reaction takes place on long exposure to moist air ; so that nitrolim forms an excellent artificial manure.

\section{The Production of Nitrides.}

In the section on ammonia, we have already mentioned one of the processes by means of which nitrides have been made of service in the nitrogen fixation problem; in the present section we may deal with another method which has been employed. In this department, as in some of the others, the invention of the electric furnace has made practicable, in technical work, methods which without its aid would never have been of any service on a large scale.

The work of Schützenberger and Colson, ${ }^{1}$ following upon that of Deville and Wöhler, ${ }^{2}$ showed that two nitrogen compounds of silicon could be obtained, one of which contained carbon in addition to the other two elements. The two substances have the following compositions :-

$$
\mathrm{Si}_{2} \mathrm{C}_{2} \mathrm{~N} \quad \mathrm{Si}_{2} \mathrm{~N}_{3}
$$

From the technical point of view it does not much matter which of these substances is formed, or even if a mixture is produced. The nitride, of course, contains a greater percentage of nitrogen than the nitride-carbide, but the difference is not of sufficient importance to make it worth while to take special precautions in order to produce the pure nitride.

Mehner has ${ }^{3}$ patented the following process. An oxide of some nitride-forming element, such as silicon, boron, or magnesium, is mixed with coal or coke and submitted to the heat

1 Schützenbeger and Colson, Compt. rend., 1882, 94, 1710.

2 Deville and Wöhler, Annalen, 1859, 110, 248.

3 Mehner, German Patent, No. 67489. 
of an electric furnace. Nitrogen is then blown through the mass. As a result, carbon reduces the oxide, yielding the element itself, which is then attacked by the nitrogen to form the nitride-

$$
\begin{aligned}
& 2 \mathrm{MgO}+\mathrm{C}=2 \mathrm{Mg}+\mathrm{CO}_{2} \\
& 6 \mathrm{Mg}+2 \mathrm{~N}_{2}=2 \mathrm{Mg}_{3} \mathrm{~N}_{2}
\end{aligned}
$$

When these nitrides are used as artificial manures, any weak acid in the soil, or even carbonic acid, is sufficient to decompose them-

$$
\mathrm{Mg}_{3} \mathrm{~N}_{2}+6 \mathrm{H}_{2} \mathrm{O}=3 \mathrm{Mg}(\mathrm{OH})_{2}+2 \mathrm{NH}_{3}
$$

Their great advantage for agricultural purposes lies in their relatively high percentage of nitrogen, which is nearly double that found in other artificial nitrogenous substances. In this way the cost of carriage is reduced. On the other hand, the cost of manufacture seems at present to be too high to allow them to compete successfully with the products of other processes. 


\section{CHAPTER III}

\section{THE PERMUTITES}

THe minerals known as zeolites are hydrated silicates of aluminium and calcium or the alkali metals. From the chemical standpoint they are of interest owing to the fact that the water-molecules in their structure appear to be very loosely attached to the rest of the complex, so loosely in fact, that they can be replaced under certain conditions by ammonia, alcohol, or sulphuretted hydrogen. Another property exhibited by the zeolites makes them of considerable technical importance. It is found that under proper conditions, the alkaline portion of their molecules can be displaced by other chemically allied groups. Thus from analcite, it is possible to produce the corresponding silver compound-

$\begin{array}{ll}\text { Analcite } & \mathrm{Na}_{2} \mathrm{O}, \mathrm{Al}_{2} \mathrm{O}_{3}, 4 \mathrm{SiO}_{2}, 2 \mathrm{H}_{2} \mathrm{O} \\ \text { Silver derivative } & \mathrm{Ag}_{2} \mathrm{O}, \mathrm{Al}_{2} \mathrm{O}_{3}, 4 \mathrm{SiO}_{2}, 2 \mathrm{H}_{2} \mathrm{O}\end{array}$

Since this replacement, in the case of artificial silicates, can be brought about by simply allowing the zeolite to remain in contact with a solution of a salt of the metal which it is desired to introduce into the silicate molecule, it is clear that the phenomenon may have a technical application in the removal of certain metals from solutions of salts.

These artificial zeolites are known commercially as permutites; and their preparation is comparatively simple. For example, in one method ${ }^{1}$ hydrated silicic acid is allowed to interact with an alkali aluminate; the mixture is then heated to complete the reaction; and finally, calcium chloride solution is employed to replace the alkali of the silicate by calcium. In this way, calcium aluminium silicates are produced which

1 Gans, D.R.-P., 1905, 174097. 
have the compositions $\mathrm{CaO}, \mathrm{Al}_{2} \mathrm{O}_{3}, 6 \mathrm{SiO}_{2}, 6 \mathrm{H}_{2} \mathrm{O}$ and $\mathrm{CaO}, \mathrm{Al}_{2} \mathrm{O}_{3}$ $4 \mathrm{SiO}_{2}, 8 \mathrm{H}_{2} \mathrm{O}$. Another method ${ }^{1}$ depends on fusing together the proper quantities of sodium sulphate, carbon, kaolin, and quartz, whereby a sodium aluminium silicate is formed which has the composition $10 \mathrm{Na}_{2} \mathrm{O}, \mathrm{Al}_{2} \mathrm{O}_{3}, 10 \mathrm{SiO}_{2}$. The actual sodium permutite which is placed on the market has the composition $\mathrm{Na}_{2} \mathrm{O}, \mathrm{Al}_{2} \mathrm{O}_{3}, 2 \mathrm{SiO}_{2}, 6 \mathrm{H}_{2} \mathrm{O}$. In order to yield the best results, it is essential that the permutite should be easily permeable by solutions.

Now let us consider the practical applications of the permutites. When sodium permutite is allowed to remain in contact with a solution of, say, calcium chloride, it is found that calcium is removed from the solution and takes the place of the sodium in the permutite molecule; whilst simultaneously the sodium of the permutite goes into solution. The result is that calcium permutite and sodium chloride solution are produced.

This at once suggests a means whereby calcium salts might be removed from hard water; but if the matter stopped there, it would be a very expensive process. At this point, advantage is taken of the law of mass action. If concentrated brine be allowed to flow over the calcium permutite, it is found that, there being a greater active mass of sodium chloride present than there is of calcium permutite, the calcium of the permutite is replaced by sodium; so that after a certain time, the sodium permutite is completely regenerated and is ready for further treatment with calcium chloride solution, from which it will remove calcium as before.

The application of this to the softening of water will now be obvious. Suppose that the water-supply of a district contains an excess of the salts of calcium, magnesium, etc., which it is necessary to remove. The flow of water is directed through a bed of sodium permutite, which removes calcium or magnesium and replaces them by sodium. In this way, both temporary and permanent hardness are destroyed. As soon as the permutite bed shows signs of losing its powers, it is shunted out of the direct flow and a stream of 10 per cent. brine is passed through it. This treatment removes the calcium from the permutite in the form of calcium chloride

1 Riedel, D.R.-P. 1906, 186630; 1907, 200931. 
solution, so that no sludge is produced; and at the same time sodium permutite is regenerated. When the regeneration is complete, the stream of brine is cut off; some pure water is run through to clear out the brine; and the permutite bed is re-shunted into the direct water-supply once more. There is practically no loss of activity in the permutite, provided that the water is filtered before entering the bed, so that no solid matter deposits itself on the permutite surface and chokes the pores in the material.

The two reactions involved in the process are as follows:$\mathrm{Na}_{2} \mathrm{O}, \mathrm{Al}_{2} \mathrm{O}_{3}, 2 \mathrm{SiO}_{2}, \mathrm{Aq}+\mathrm{CaSO}_{4}$

Sodium permutite.

$$
=\mathrm{CaO}, \mathrm{Al}_{2} \mathrm{O}_{3}, 2 \mathrm{SiO}_{2}, \mathrm{Aq}+\mathrm{Na}_{2} \mathrm{SO}_{4}
$$

Calcium permutite.

$\mathrm{CaO}, \mathrm{Al}_{2} \mathrm{O}_{3}, 2 \mathrm{SiO}_{2}, \mathrm{Aq}+2 \mathrm{NaCl}=\mathrm{Na}_{2} \mathrm{O}, \mathrm{Al}_{2} \mathrm{O}_{3}, 2 \mathrm{SiO}_{2}, \mathrm{Aq}+\mathrm{CaCl}_{2}$ Calcium permutite. Sodium permutite.

By a similar series of reactions, the solution of a potassium salt may be converted into a sodium salt solution; and this method is employed in the case of molasses. Since molasses is naturally contaminated with potassium salts which impart an undesirable taste to the product, the permutite process is utilized to replace the potassium by sodium, which is not so objectionable.

The removal of free alkali from water can also be accomplished by a modification of the permutite process. Sodium permutite in presence of acids gives up its sodium, which is replaced by hydrogen. This hydrogen permutite possesses the property of absorbing alkali from alkaline solutions, forming sodium permutite which, by further treatment with acid, can be re-converted into hydrogen permutite; so that in this case also the process is a cyclic one.

Another troublesome impurity in some sources of water is iron. This also may be removed by the permutite process, though the method is more complicated than in the examples given above. The first step is the preparation of a manganese permutite, which is obtained from calcium permutite by the action of manganese chloride-

$$
\begin{aligned}
& \mathrm{CaO}, \mathrm{Al}_{2} \mathrm{O}_{3}, 2 \mathrm{SiO}_{2} \\
& \text { Calcium permutite. }
\end{aligned}
$$


The next stage in the process is the introduction of manganese in a higher state of oxidation; whilst simultaneously calcium takes its place in the permutite molecule. This change is accomplished by treating the manganese permutite with calcium permanganate-

$$
\begin{aligned}
\mathrm{MnO}, \mathrm{Al}_{2} \mathrm{O}_{3}, 2 \mathrm{SiO}_{2}+\mathrm{Ca}\left(\mathrm{MnO}_{4}\right)_{2}=\mathrm{CaO}, \mathrm{Al}_{2} \mathrm{O}_{3}, & 2 \mathrm{SiO}_{2} \\
& +\mathrm{MnO}, \mathrm{Mn}_{2} \mathrm{O}_{7}
\end{aligned}
$$

The manganese oxides are precipitated upon the permutite, forming a dark-coloured mass. Now if any iron be present in the water, it is at once oxidized by this material; and iron oxide is deposited from the solution. Apparently the action is partly catalytic. Special precautions are necessary to avoid the permutite bed becoming choked with particles of iron oxide.

It will be seen that even when no iron is present, this method may still be of service; for it enables water to be sterilized by means of a permanganate without any risk of manganese passing into the supply. Permanganate is added to the water; any bacteria present are thus destroyed; and the manganese is removed by passing the liquid through a permutite bed. Organic impurities may be similarly oxidized.

The application of the permutite process to the problem of gold-extraction has been suggested. If a ferrous or stannous permutite be submitted to the action of a gold salt solution, the gold is removed and precipitated on the permutite surface in the form of purple of Cassius.

Finally, we may refer to the utilization of permutites in the preparation of certain salts. Suppose that from potassium chloride and ammonium carbonate it is desired to produce potassium carbonate. A potassium permutite is formed; and this is then treated with ammonium carbonate solution. Potassium is liberated from the permutite and gives the required potassium carbonate.

It will be seen that the applications of the permutites are far-reaching even at the present day; and it is probable that in the near future they will be even further extended. 


\section{CHAPTER IV}

\section{THE PEROXIDES AND THE PER-ACIDS}

\section{Hydrogen Peroxide.}

OwING to its instability, hydrogen peroxide is only to be obtained in a pure state after a long series of operations. In the first place, a solution is prepared in the following manner. ${ }^{1}$ Sodium peroxide, produced by the oxidation of sodium, is added to 20 per cent. sulphuric acid, the reacting materials being kept at a low temperature during the process. Hydrogen peroxide and sodium sulphate are thus formed; and the sodium sulphate in part separates out from the solution, taking with it a certain amount of water in the form of water of crystallization and thus leaving the solution more concentrated. The solid is then removed by filtration; and the solution is concentrated in vacuo; or, as an alternative, ${ }^{2}$ alcohol may be added, which precipitates more of the sulphate and yields an alcoholic solution of the peroxide. This last method has the disadvantage that explosive ethyl peroxides may be formed during the process. Sometimes hydrofluoric $\operatorname{acid}^{3}$ is used to decompose the sodium peroxide; and the removal of the acid from the solution is accomplished by taking advantage of the low solubility of certain double fluorides. Thereafter the peroxide solution is concentrated, as before, by distillation under reduced pressure. When an 80 per cent. solution has been obtained, it is frozen, and practically pure hydrogen peroxide separates out. ${ }^{4}$ If an absolutely pure product is required, it can only be prepared by a long process of repeated fractional distillation.

1 Merck, D.R.-P., 152173.

2 Dony-Henault, French Patent, 1909, 403294.

3 Hulin-Schumann, D.R.-P., 132090; and D.R.-P., 253287.

4 Arhle, J.pr. Chem., 1909, (2) 79, 129. 
Pure hydrogen peroxide is fairly stable, and can be kept for a considerable time without decomposition. The solutions, on the other hand, are much more easily decomposed. Roughness of the walls of the containing vessel, the presence of free alkali, salts of heavy metals or suspended solid matter hasten the break-down. Colloidal metals especially have a marked influence upon the rate of decomposition. ${ }^{1}$

Hydrogen peroxide is a powerful oxidizing agent; and it is therefore somewhat astonishing to find that it is capable of acting as a reducing agent in certain cases. Oxides of silver, gold, and platinum are reduced by it to the metallic state-

$$
\mathrm{H}_{2} \mathrm{O}_{2}+\mathrm{Ag}_{2} \mathrm{O}=\mathrm{Ag}_{2}+\mathrm{H}_{2} \mathrm{O}+\mathrm{O}_{2}
$$

On the other hand, many basic oxides are converted into peroxides-

$$
\mathrm{H}_{2} \mathrm{O}_{2}+\mathrm{Ba}(\mathrm{OH})_{2}=\mathrm{BaO}_{2}+2 \mathrm{H}_{2} \mathrm{O}
$$

Hydrogen peroxide has certain acidic properties; for towards some salts it behaves as if it were an acid-

$$
\mathrm{Na}_{2} \mathrm{CO}_{3}+\mathrm{H}_{2} \mathrm{O}_{2}=\mathrm{Na}_{2} \mathrm{O}_{2}+\mathrm{CO}_{2}+\mathrm{H}_{2} \mathrm{O}
$$

The constitution of hydrogen peroxide is still in dispute. Three formulæ have been proposed-

$$
\mathrm{H}-\mathrm{O}-\mathrm{O}-\mathrm{H}
$$

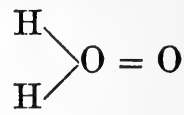

(II.)

$$
\mathrm{H}-\mathrm{O} \equiv \mathrm{O}-\mathrm{H}
$$

(III.)

The first of these is the oldest. Its main defect is that it fails to express the fact that hydrogen peroxide splits off one atom of oxygen readily, but does not part with the other. The second suggestion is due to Kingzett, ${ }^{2}$ and it expresses the readiness with which water is formed from the peroxide. Bruhl $^{3}$ is responsible for the third structure, which he found to agree with the refractivity of the substance better than either of the others. When the variety of reactions given by hydrogen peroxide is examined, it seems probable that the substance can exist in the tautomeric condition and that its

1 Bredig, Zeitsch. physikal. Chem., 1899, 31, 258; 1901, 37, 1, 323; 1901, 38, 122.

2 Kingzett, Chem. News, 1884, 46, 141.

3 Bruhl, Ber., 1895, 28, 2837. 
structure is determined by the outside reagent employed upon it. Possibly its constitution varies from (I.) to (II.) according to the conditions under which it reacts.

\section{The Metallic Peroxides.}

Oxides containing two atoms of oxygen are divisible into two classes: (1) those which liberate hydrogen peroxide when treated with acids; and (2) those which yield no hydrogen peroxide. Clearly the two classes must have different constitutions; and it is usual to distinguish them by naming the first type peroxides, whilst the second set are termed dioxides-

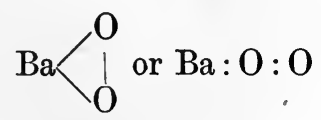

Barium peroxide.<smiles>O=[14C]=O</smiles>

Manganese dioxide.

We are here concerned only with the true peroxides.

Since in hydrogen peroxide there are two hydrogen atoms replaceable by metals, it is clear that two substitution products may be expected-

$$
\mathrm{Me}-\mathrm{O}-\mathrm{O}-\mathrm{H} \text { and } \mathrm{Me}-\mathrm{O}-\mathrm{O}-\mathrm{Me}
$$

The mono-substitution products, termed hydroperoxides, are not of any technical importance. Tafel ${ }^{1}$ prepared what appears to be the sodium compound, $\mathrm{Na} . \mathrm{O} . \mathrm{O} . \mathrm{H}$, by shaking sodium peroxide with an ice-cold mixture of alcohol and a concentrated mineral acid (or even with alcohol alone). The product is a white, sandy, unstable powder which on heating evolves oxygen with explosive violence. The same substance has been obtained by Wolffenstein ${ }^{2}$ by the action of sodium ethylate upon hydrogen peroxide-

$$
\mathrm{C}_{2} \mathrm{H}_{5} \cdot \mathrm{O} . \mathrm{Na}+\mathrm{H} . \mathrm{O} . \mathrm{O} . \mathrm{H}=\mathrm{C}_{2} \mathrm{H}_{5} . \mathrm{OH}+\mathrm{Na} . \mathrm{O} . \mathrm{O} . \mathrm{H}
$$

Ammonium hydroperoxide is produced analogously ${ }^{3}$ by the action of hydrogen peroxide upon ammonia in ethereal solution at $-40^{\circ} \mathrm{C}$. The calcium and barium compounds are

1 Tafel, Ber., 1894, 27, 816.

2 Wolffenstein, D.R.-P., 196369.

3 Melikoff and Pissarjewsky, Ber., 1898, 31, 446. 
formed ${ }^{1}$ by the action of hydrogen peroxide in excess upon the hydrates of the metals-

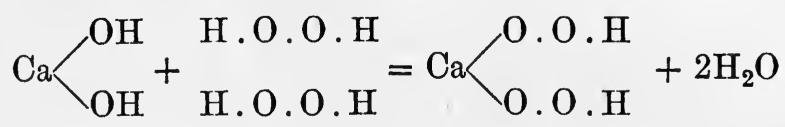

Turning now to the disubstitution products of hydrogen peroxide, the sodium compound is of some technical value. It is prepared ${ }^{2}$ by oxidation of metallic sodium with air at a temperature of about $300^{\circ} \mathrm{C}$. The air is first freed from water and carbon dioxide and is then driven down a heated passage. Up this passage the sodium is pushed, being contained in aluminium cases mounted on trucks; and the method is so contrived that fresh sodium only meets the air-current after the latter has already been partially exhausted by acting upon half-converted sodium. In other words, fresh sodium is added to the line of trucks at the point furthest from where the air enters the passage. In this way violent reactions are avoided.

Sodium peroxide reacts violently with water, yielding various hydrates. With organic matter, it forms explosive mixtures, though the peroxide itself seems devoid of explosive properties. It is employed commercially as an absorbent for carbon dioxide; as an oxidizing agent; and as a bleacher.

When we come to the peroxides of divalent metals, several formulæ can be suggested to express their structure-

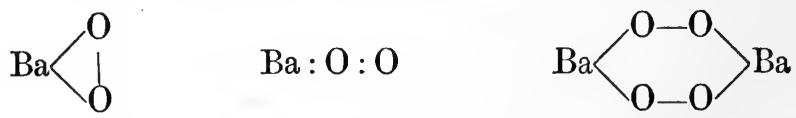

Since it is impossible to determine the molecular weight of such substances, we cannot even decide definitely whether they should be regarded as containing one or two atoms of the metal. The first of the above formulæ is derived from the old formula for hydrogen peroxide; the second one expresses the ease with which the peroxides yield oxygen; whilst the third structure is supported by the fact that compounds of the type $\mathrm{Ba}\left(\mathrm{O} . \mathrm{O} . \mathrm{C}_{2} \mathrm{H}_{5}\right)_{2}$ have been isolated. ${ }^{3}$

In general, the peroxides of divalent elements differ from

1 Schöne, Annalen, 1878, 192, 257.

2 D.R.-P., 224480.

3 Baeyer and Villiger, Ber., 1911, 44, 738. 
those of the monovalent metals in that the former can be prepared from oxides, whilst the latter can only be obtained by the direct oxidation of the metal.

Barium peroxide is the most valuable representative of its class from the commercial standpoint. Its preparation and adaptation in the Brin oxygen process require no detailed description here.

\section{The General Character of the Per-acids and their Salts.}

Chemical nomenclature is by no means ideal; and in the case of the per-acids it is especially confusing, owing to a mingling of an older with a newer terminology. For example, when we speak of perchloric acid, we merely imply that this is an acid derived from chlorine in the highest state of oxidation which we find displayed in that class of substances. The name persulphuric acid, on the other hand, is given to the compound on account of the fact that this acid is derived from hydrogen peroxide by substitution. Thus the reader must be on his guard so as not to confuse the older "per-acids" with the newer type which is now regarded as the true claimant to the title.

The true per-acids, then, are those acids which are derived from hydrogen peroxide by the substitution of an acidic radicle for one or more hydrogen atoms-

\section{$\mathrm{O}: \mathrm{B}-\mathrm{OH}$}

Boric acid.
$\mathrm{O}: \mathrm{B}-\mathrm{O} . \mathrm{O} . \mathrm{H}$

Perboric acid.

$\mathrm{HO} . \mathrm{SO}_{2}-\mathrm{OH} \mathrm{HO} . \mathrm{SO}_{2}-\mathrm{O} . \mathrm{O} . \mathrm{H} \mathrm{HO} . \mathrm{SO}_{2}-\mathrm{O} . \mathrm{O}-\mathrm{SO}_{2} . \mathrm{OH}$ Sulphuric acid. Permonosulphuric acid.

Perdisulphuric acid.

As might be expected, the elements capable of giving rise to per-acids are confined to the third, fourth, fifth, and sixth groups of the Periodic Table. In Group III. boron stands alone, though lanthanum has been suspected of per-acid formation. ${ }^{1}$ In Group IV. we find carbon, titanium, zirconium, and tin. In Group V. there are nitrogen, phosphorus, vanadium, columbium, and tantalum; whilst in Group VI. the per-acid-forming elements are sulphur, chromium, selenium, molybdenum, tungsten, and uranium. It will be noticed that

1 See Melikoff and Pissarjewsky, Zeitsch. anorgan. Chem., 1899, 21, 70. 
the number of elements per group increases as we go across the table, beginning with one in Group III. and ending with six in Group VI. Also, if we exclude the elements of the short periods, all the others which we have mentioned-with the exception of tin and selenium-belong to the even series. Finally, it appears that the stability of the per-acids in each group increases with an increase in the atomic weight of the parent element.

In the succeeding sections of this chapter, a brief account will be given of some of the per-acids and their salts; but for full details the reader must be referred to other works. ${ }^{1}$

\section{The Persulphuric Acids and the Persulphates.}

The history of the persulphuric acids dates back to Faraday; but it is somewhat confusing, owing to the number of apparently contradictory data which were accumulated; and in this section it will be best to omit the earlier investigations and deal with the subject in non-chronological order. ${ }^{2}$

The following apparatus is used in the preparation of potassium persulphate: ${ }^{3}$ A platinum dish forms the anode. This is filled with a saturated solution of potassium sulphate in dilute sulphuric acid; and into it is dipped a porous pot containing dilute sulphuric acid. In the porous pot lies the cathode, which takes the form of a platinum wire. It is essential that the cathode surface should be small; for with a large cathode the yields are poor. ${ }^{4}$ The platinum vessel forming the anode is kept cool by a stream of water passing over the outside. A current of about three ampères is used; and after a day or two potassium persulphate begins to crystallize out. Ammonium persulphate can be obtained in an analogous manner.

It appears that the stages involved in the persulphate formation are as follows. In the first place, the potassium sulphate reacts with the free sulphuric acid present to form potassium hydrogen sulphate. This then ionizes into $\mathrm{H} \cdot$ and

1 See especially Price, The Per-acids and their Salts.

2 For a summary of the history of the subject, see Price, The Per-acids and their Salts, pp. $10 \mathrm{ff}$.

3 Marshall, Proc. Roy. Soc. Edin., 1891, 18, 63 ; Trans., 1891, 59, 771.

4 Marshall, J. Soc. Chem. Ind., 16, 396. 
$\mathrm{KSO}_{4}{ }_{4}$. Molecular hydrogen appears at the cathode; and the $\mathrm{KSO}_{4}^{\prime}$ ions join together in pairs to form $\mathrm{K}_{2} \mathrm{~S}_{2} \mathrm{O}_{8}$.

Now when sulphuric acid is added to a persulphate, we should expect to find the parent persulphuric acid liberated; but the matter is not quite so simple in practice. If dilute sulphuric acid be used, the resulting solution oxidizes aniline direct to aniline black; but if the persulphate be dissolved in concentrated sulphuric acid and the liquid be then diluted, it is found that the addition of aniline produces, not aniline black, but nitrosobenzene. Evidently, two different substances can be formed: one being produced by the action of dilute acid on the persulphate; the other being formed by the action of concentrated acid on the salt.

It is now known that there are actually two persulphuric acids. The one obtained from a persulphate and dilute acid is termed perdisulphuric acid and has the formula $\mathrm{H}_{2} \mathrm{~S}_{2} \mathrm{O}_{8}$; whilst the other, produced when concentrated sulphuric acid is used, is termed Caro's acid or permonosulphuric acid, and has the composition $\mathrm{H}_{2} \mathrm{SO}_{5}$.

Perdisulphuric acid can be obtained in the anhydrous state by allowing one molecule of hydrogen peroxide to act upon two molecules of chlorosulphonic acid, the mixture being kept cool. From this it would appear that perdisulphuric acid has the constitution shown below-

$$
\underset{\mathrm{O}-\mathrm{H}}{\mathrm{O}-\mathrm{H}}+\underset{\mathrm{Cl} \cdot \mathrm{SO}_{2} \cdot \mathrm{OH}}{\mathrm{Cl} \cdot \mathrm{SO}_{2} \cdot \mathrm{OH}}=\stackrel{\mathrm{O}-\mathrm{SO}_{2}-\mathrm{OH}}{\mathrm{O}-\mathrm{SO}_{2}-\mathrm{OH}}+2 \mathrm{HCl}
$$

Caro's acid can be produced, ${ }^{1}$ in addition to the method already given, by two reactions : by the electrolysis of a fairly concentrated solution of sulphuric acid; or by the action of concentrated sulphuric acid upon concentrated hydrogen peroxide. Its composition was for a long time in doubt, the two formulæ $\mathrm{H}_{2} \mathrm{~S}_{2} \mathrm{O}_{9}$ and $\mathrm{H}_{2} \mathrm{SO}_{5}$ being proposed. The proof which enabled a choice to be made between the two suggestions is due to Willstätter and Hauenstein. ${ }^{2}$ Sodium persulphate was treated with concentrated sulphuric acid and the resulting persulphuric acid was neutralized with sodium carbonate. The

1 Baeyer and Villiger, Ber., 1901, 34, 853.

2 Willstätter and Hauenstein, Ber., 1909, 42, 1839. 
sodium salt of Caro's acid was thus formed. When this was treated with benzoyl chloride in presence of sufficient sodium hydroxide to keep the solution neutral, a benzoyl derivative of Caro's acid was formed. Analysis of this proved that for each sulphur atom in the compound there was one benzoyl group present. Now if the formula of the acid be $\mathrm{H}_{2} \mathrm{~S}_{2} \mathrm{O}_{9}$, the benzoyl derivative will contain two benzoyl radicles-one for each sulphur atom according to the analysis. Its formula will therefore be $\left(\mathrm{C}_{6} \mathrm{H}_{5} \cdot \mathrm{CO}\right)_{2} \mathrm{~S}_{2} \mathrm{O}_{9}$, and it should contain no metallic atom. If Caro's acid be $\mathrm{H}_{2} \mathrm{SO}_{5}$, on the other hand, there is only one sulphur atom present; and therefore the benzoyl compound can contain only a single benzoyl radicle. Its formula would thus be $\mathrm{C}_{6} \mathrm{H}_{6} \cdot \mathrm{CO}^{\mathrm{S}} \mathrm{SO}_{5} . \mathrm{H}$; and it should be capable of forming a potassium salt. The product does actually form a potassium salt $\mathrm{C}_{6} \mathrm{H}_{5} \cdot \mathrm{CO} . \mathrm{SO}_{5} . \mathrm{K}$, which establishes the formula of Caro's acid as $\mathrm{H}_{2} \mathrm{SO}_{5}$. Its constitution is probably expressed by : $\mathrm{HO} . \mathrm{SO}_{2} \cdot \mathrm{O} \cdot \mathrm{OH}$, which evidently represents a molecule of hydrogen peroxide wherein one hydrogen atom is replaced by a sulphonic radicle. , On this account, Caro's acid is correctly termed permonosulphuric acid. The constitution of ordinary "persulphuric acid," $\mathrm{H}_{2} \mathrm{~S}_{2} \mathrm{O}_{8}$, has been shown to be analogous to that of Caro's acid. It also is derived from hydrogen peroxide by replacing hydrogen by the sulphonic group; but in this case both the hydrogen atoms are replaced: $\mathrm{HO} \mathrm{SO}_{2} \cdot \mathrm{O} . \mathrm{O} . \mathrm{SO}_{2} \cdot \mathrm{OH}$. The acid is therefore known as perdisulphuric acid.

The relations between perdisulphuric acid and its decomposition products were not made clear until a considerable amount of research had been carried out, most of which, at the time, appeared only to make confusion worse than before. It was finally established that the facts of the case were as follows. When perdisulphuric acid is freshly prepared by the electrolytic method it exists alone in solution; and hence the solution containing it shows the reactions of pure perdisulphuric acid. For example, it bleaches indigo and oxidizes ferrocyanides; but with chromic acid and potassium permanganate it shows none of the reactions attributable to hydrogen peroxide. The ratio between "sulphuric acid formed" and "active oxygen liberated" in its reactions is $2: 1$. If the solution be allowed to stand for a time before being tested, 
it is found that its character alters. In any reaction to which it is subjected, the ratio of "sulphuric acid formed" to "active oxygen liberated" is now $1: 1$. Finally, on long standing, or in presence of colloidal metals, the solution undergoes yet another change. Hydrogen peroxide is formed, which reacts with the remaining acid, giving sulphuric acid and free oxygen. These various reactions are represented in the following equations:-

$$
\begin{aligned}
& \mathrm{H}_{2} \mathrm{~S}_{2} \mathrm{O}_{8}+\mathrm{H}_{2} \mathrm{O}=\mathrm{H}_{2} \mathrm{SO}_{4}+\mathrm{H}_{22} \mathrm{SO}_{5} \\
& \mathrm{H}_{2} \mathrm{SO}_{5}+\mathrm{H}_{2} \mathrm{O}=\mathrm{H}_{2} \mathrm{SO}_{4}+\mathrm{H}_{2} \mathrm{O}_{2} \\
& \mathrm{H}_{2} \mathrm{SO}_{5}+\mathrm{H}_{2} \mathrm{O}_{2}=\mathrm{H}_{2} \mathrm{SO}_{4}+\mathrm{H}_{2} \mathrm{O}^{2}+\mathrm{O}_{2}
\end{aligned}
$$

The persulphates derived from perdisulphuric acid are much more stable than the parent substance, those which crystallize anhydrous being less liable to decomposition than those which contain water of crystallization. All of them are soluble in water. In solution, they gradually decompose, like the parent acid, yielding hydrogen sulphates and oxygen-

$$
\mathrm{K}_{2} \mathrm{~S}_{2} \mathrm{O}_{8}+\mathrm{H}_{2} \mathrm{O}=2 \mathrm{KHSO}_{4}+\mathrm{O}
$$

The most striking property of the persulphates, however, is their oxidizing power. A persulphate liberates free halogen from halides-

$$
\mathrm{K}_{2} \mathrm{~S}_{2} \mathrm{O}_{8}+2 \mathrm{KI}=2 \mathrm{~K}_{2} \mathrm{SO}_{4}+\mathrm{I}_{2}
$$

and it is found that this reaction is catalysed by the presence of iron or copper salts. 1 Ferrous salts, when treated with a persulphate, are oxidized to the ferric state; and this reaction forms a convenient method for persulphate estimation, in conjunction with the usual permanganate titration. The oxidation of manganese salts to permanganates forms a simple mode of detecting the presence of a persulphate, since the colour of the permanganate can be seen in extremely dilute solutions.

One of the most interesting reactions of the persulphates is that shown when their solutions act upon metals. ${ }^{2}$ No marked evolution of gas occurs; and the action appears to take the course shown below-

$$
\mathrm{K}_{2} \mathrm{~S}_{2} \mathrm{O}_{8}+\mathrm{Zn}=\mathrm{K}_{2} \mathrm{SO}_{4}+\mathrm{ZnSO}_{4}
$$

1 Price, Zeitsch. physikal. Chem., 1898, 17, 459.

2 Marshall, J. Soc. Chem. Ind., 1897, 16, 396; Tarugi, Gazzetta, 1903, 33 (i.), 127 ; Turrentine, J. Physical. Chem., 1907, 11, 623 ; Levi, Migliorini, and Ercolini, Gazzetta, 1908, 38 (i.), 583. 
Magnesium forms an exception to the general rule; for when it is treated with ammonium persulphate solution a violent reaction takes place and ammonia is evolved.

The persulphates have found a most extensive application as oxidizing agents. In analytical work they are employed in iron and steel analyses for the estimation of manganese or chromium; whilst in organic chemistry their uses are too numerous to mention. Caro's acid, especially, has proved of value in organic reactions, as it is one of the most easily regulated oxidizing agents which we possess.

\section{The Per-acids of Group $V$.}

Neither of these acids has been isolated in a pure state; and the evidence for their existence is therefore indirect.

When nitrogen pentoxide interacts with anhydrous hydrogen peroxide, the mixture being kept well cooled, a colourless liquid smelling like bleaching-powder is obtained. ${ }^{1}$ The aqueous solution of this substance liberates iodine from potassium iodide and oxidizes aniline to nitrosobenzene. In dilute solutions, the compound is hydrolysed, hydrogen peroxide and nitric acid being liberated. The reaction may be assumed to take the following course :-

$$
\mathrm{N}_{2} \mathrm{O}_{5}+\mathrm{H}_{2} \mathrm{O}_{2}=\mathrm{HNO}_{3}+\mathrm{HO} \text {.O. } \mathrm{NO}_{2}
$$

A solution having the same properties is produced when a very dilute aqueous solution of nitrous acid is oxidized with hydrogen peroxide $^{2}-$

$$
\mathrm{H}_{2} \mathrm{O}_{2}+\mathrm{HNO}_{2}=\mathrm{HNO}_{4}+2 \mathrm{H}_{2} \mathrm{O}
$$

Further evidence on the possibility of the existence of pernitric acids is to be found in the results of electrolysing silver salts $;^{3}$ but up to the present it is the subject of dispute.

1 d'Ans and Friederich, Zeitsch. anorgan. Chom., 1911, 73, 344 ; d'Ans, Zeitsch. Elektrochem., 1911, 17, 850.

2 Raschig, Zeitsch. angew. Chem., 1904, 17, 1419; Ber., 1907, 40, 4585.

3 See Berthelot, Compt. rend., 1880, 90, 653; Šulc, Zeitsch. anorgan. Chem., 1896, 12, 89, 180 ; 1900, 24, 305; Mulder and Heringa, Rec. trav. chim., 1896, 15, 235 ; Mulder, ibid., 1897, 16, 57 ; 1898, 17, 129 ; 1899, 18, 91 ; 1903, 22, 385, 388, 405; Tanatar, Zeitsch. anorgan. Chem., 1901, 28, 331; Bose, Zeitsch. anorgan. Chem., 1905, 44, 237 ; Watson, Trans., 1906, 89, 578; Brauner and Kužma, Ber., 1907, 40, 3371 ; Luther and Pokorny, Zeitsch. anorgan. Chem., 1908, 57, 290 ; Baborovsky and Kužma, Zeitsch. physikal. Chem., 1909, 67, 48 ; Bose, ibid., 1909, 68, 383. 
The evidence for the existence of perphosphoric acid is stronger. It is not obtained by the action of hydrogen peroxide upon phosphoric acid, nor by the electrolysis of phosphoric acid or the phosphates; but when 30 per cent. hydrogen peroxide is treated with phosphorus pentoxide, meta- or pyrophosphoric acid, a solution is obtained which possesses strong oxidizing properties which cannot be accounted for merely by the presence of hydrogen peroxide. ${ }^{1}$ The diluted solution will even oxidize manganese salts to permanganic acid. It appears probable that $\mathrm{H}_{3} \mathrm{PO}_{5}$ is present.

Pervanadic acid, percolumbic acid, and pertantalic acid are all known. The two first are obtained by the action of hydrogen peroxide upon the corresponding lower acid, whilst pertantalic acid can be prepared from its potassium salt by the action of sulphuric acid.

\section{The Percarbonates.}

Potassium and rubidium percarbonates are obtained by electrolysing concentrated solutions of the corresponding carbonates in an apparatus similar to that employed in the case of the persulphates. ${ }^{2}$ The temperature must be kept low; and hence the method is inapplicable to carbonates which do not form strong solutions at zero or below it.

The course of the reaction appears to be similar to that which is observed in the persulphates. Potassium carbonate dissociates into the ions $\mathrm{K}$. and $\mathrm{KCO}_{3}$; and then two of the latter unite to form $\mathrm{K}_{2} \mathrm{C}_{2} \mathrm{O}_{6}$. From this it seems probable that the constitution of potassium percarbonate is that which is expressed below-

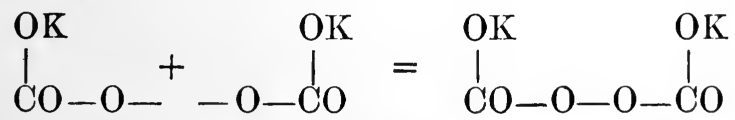

An examination of this formula will show that it is derived from hydrogen peroxide by replacing the hydrogens by two

1 Schmidlin and Massini, Ber., 1910, 43, 1162 ; d'Ans and Friederich, Ber., 1910, 43, 1880 ; Zeitsch. anorgan. Chem., 1911, 73, 343 ; d'Ans, Zeitsch. Elektrochem., 1911, 17, 850.

2 Constam and Hansen, Zeitsch. Elektrochem., 1896, 3, 137. 
carbonic acid nuclei and then forming the di-potassium salt of the acid thus produced. In other words, this per-acid is, properly speaking, perdicarbonic acid, analogous to perdisulphuric acid.

The action of 3 per cent. hydrogen peroxide upon sodium carbonate gives rise to a substance ${ }^{1}$ having the composition $\mathrm{Na}_{2} \mathrm{CO}_{4}, 1 \frac{1}{2} \mathrm{H}_{2} \mathrm{O}$ or $\mathrm{Na}_{2} \mathrm{CO}_{3}, \mathrm{H}_{2} \mathrm{O}_{2}, \frac{1}{2} \mathrm{H}_{2} \mathrm{O}$. Whether this is a true percarbonate or merely, as the second formula suggests, a carbonate with hydrogen peroxide of crystallization, is not clearly established. True per-acids liberate iodine from potassium iodide instantaneously; but Tanatar's salt is almost without immediate effect upon iodides. The evidence, therefore, is against it being a true percarbonate.

The action of carbon dioxide upon sodium peroxide and sodium hydroperoxide is interesting. Either one or two molecules of carbon dioxide can react with the peroxide, giving the following results :-

$$
\begin{aligned}
& \text { (1) } \mathrm{Na}_{2} \mathrm{O}_{2}+\mathrm{CO}_{2}=\mathrm{Na}_{2} \mathrm{CO}_{4} \\
& \text { (2) } \mathrm{Na}_{2} \mathrm{O}_{2}+2 \mathrm{CO}_{2}=\mathrm{Na}_{2} \mathrm{C}_{2} \mathrm{O}_{6}
\end{aligned}
$$

At first sight, it seems probable that the compound $\mathrm{Na}_{2} \mathrm{C}_{2} \mathrm{O}_{6}$ is derived from the same acid as $\mathrm{K}_{2} \mathrm{C}_{2} \mathrm{O}_{6}$ which is prepared by the electrolytic method. An examination of the effect of the two substances on potassium iodide shows that this is not the case. The electrolytically prepared salt liberates iodine quantitatively, according to its nature as a true per-acid: but the sodium compound prepared from sodium peroxide only liberates half the theoretical quantity of iodine. Since the potassium salt has the structure (I.), the only probable constitution for the sodium salt is (II.)-

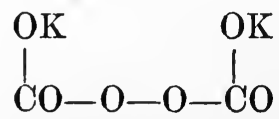

(I.)

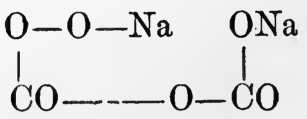

(II.)

The compound $\mathrm{Na}_{2} \mathrm{CO}_{4}$ appears to be sodium permonocarbonate, analogous to sodium permonosulphate-

$$
\mathrm{NaO} \text {. O.CO.O.Na } \mathrm{NaO} \text {. O. } \mathrm{SO}_{2} \cdot \mathrm{ONa}
$$

The action of carbon dioxide upon sodium hydroperoxide 
also takes two courses, though -in this case only one molecule of carbon dioxide is involved, and either one or two molecules of hydroperoxide may take part in the reaction-

$$
\begin{aligned}
\mathrm{Na} . \mathrm{O} \cdot \mathrm{O} \cdot \mathrm{H}+\mathrm{CO}_{2} & =\mathrm{NaHCO}_{4} \\
2 \mathrm{Na} \text { OO.O.H } & \mathrm{OHO} \mathrm{CO}_{2}=\mathrm{Na}_{2} \mathrm{CO}_{5}, \mathrm{H}_{2} \mathrm{O}
\end{aligned}
$$

The two products of the reactions are apparently compounds formed by the addition of hydrogen peroxide to the substances $\mathrm{Na}_{2} \mathrm{CO}_{4}$ and $\mathrm{Na}_{2} \mathrm{C}_{2} \mathrm{O}_{6}$ which were produced in reactions (1) and (2) above.

The properties of potassium percarbonate may now be described.1 It is a sky-blue ${ }^{*}$ hygroscopic powder, which, when gently warmed, decomposes according to the equation-

$$
2 \mathrm{~K}_{2} \mathrm{C}_{2} \mathrm{O}_{6}=2 \mathrm{~K}_{2} \mathrm{CO}_{3}+2 \mathrm{CO}_{2}+\mathrm{O}_{2}
$$

Its solution at ordinary temperatures liberates hydrogen peroxide and hence produces oxygen-

$$
\mathrm{K}_{2} \mathrm{C}_{2} \mathrm{O}_{6}+2 \mathrm{H}_{2} \mathrm{O}=2 \mathrm{KHCO}_{3}+\mathrm{H}_{2} \mathrm{O}_{2}
$$

The presence of potassium hydroxide accelerates the reaction, the decomposition taking place at zero with the production of hydrogen peroxide and potassium carbonate. With dilute sulphuric acid in the cold, hydrogen peroxide and carbon dioxide are liberated quantitatively-

$$
\mathrm{K}_{2} \mathrm{C}_{2} \mathrm{O}_{6}+\mathrm{H}_{2} \mathrm{SO}_{4}=\mathrm{K}_{2} \mathrm{SO}_{4}+2 \mathrm{CO}_{2}+\mathrm{H}_{2} \mathrm{O}_{2}
$$

Potassium percarbonate is a good oxidizing agent in certain cases, such as the oxidation of lead sulphide to lead sulphate; but it can also play the part of a reducing agent. For example, when it is treated with manganese dioxide, lead dioxide or silver oxide, oxygen is evolved and the corresponding metallic carbonate is formed.

In technical practice, potassium percarbonate is utilized for bleaching purposes and also as a "hypo-killer" in photography under the name of "Antihypo." Sodium permonocarbonate is utilized as a source of hydrogen peroxide and also in medicine as an antiseptic.

1 Wolffenstein and Peltner, Ber., 1908, 41, 280.

* Rubidium percarbonate is colourless. 


\section{The Perborates.}

Perboric acid, $\mathrm{HBO}_{3}$, does not exist in the free state; but many of its salts are known; and of these, from the commercial standpoint, sodium perborate is the most important.

Sodium perborate ${ }^{1}$ is manufactured by any of the three reactions represented below which take place in aqueous solutions-

(1) $2 \mathrm{NaOH}+\mathrm{Na}_{2} \mathrm{~B}_{4} \mathrm{O}_{7}, 10 \mathrm{H}_{2} \mathrm{O}+4 \mathrm{H}_{2} \mathrm{O}_{2}+\mathrm{H}_{2} \mathrm{O}$

(2) $\mathrm{Na}_{2} \mathrm{O}_{2}+\mathrm{H}_{3} \mathrm{BO}_{3}+\mathrm{HCl}+2 \mathrm{H}_{2} \mathrm{O}=\mathrm{NaBO}_{3}, 4 \mathrm{H}_{2} \mathrm{O}+\mathrm{NaCl}$ (3) $\mathrm{Na}_{2} \mathrm{O}_{2}+\mathrm{H}_{2} \mathrm{O}_{2}+2 \mathrm{H}_{3} \mathrm{BO}_{3}=2 \mathrm{NaBO}_{3}, 4 \mathrm{H}_{2} \mathrm{O}$

The possibility of preparing perborates by electrolysing concentrated solutions of orthoborates appears to be still in doubt. Tanatar, ${ }^{2}$ Pouzenc, ${ }^{3}$ Bruhat and Dubois ${ }^{4}$ claim that this method can be used; but Constam and Bennet ${ }^{5}$ state that no perborates are thus formed.

When preserved with customary precautions, sodium perborate decomposes only slowly, though the breakdown may be hastened by the presence of impurities. At times, however, it is liable to an unexplained type of decomposition which may take place after the substance has been kept quite unaffected for months. In this reaction, the perborate liquefies and froths; and its behaviour resembles to some extent that of decomposing hydrogen peroxide.

When sufficiently diluted, aqueous solutions of sodium perborate behave almost as if they contained free hydrogen peroxide, free boric acid, and free sodium hydrate; and since alkaline solutions of hydrogen peroxide are readily decomposed even at ordinary temperatures, it is clear that any reaction involving sodium perborate must be carried through rapidly before the evolved oxygen escapes.

The commerical applications of sodium perborate depend upon its power of bleaching. In the laundry industry it has

1 Tanatar, Zeitsch. physikal. Chem., 1898, 25, 265; 1898, 26, 132, 451; 1899, 29, 152 ; Melikoff and Pissarjewsky, Ber., 1898, 31, 678; Jaubert, Compt. rend., 1904, 189, 796; Bruhat and Dubois, ibid., 1905, 140, 506.

2 Tanatar, Zeitsch. physikal. Chem., 1898, 26, 132.

3 Pouzenc, French Patent, 411258.

4 Bruhat and Dubois, Compt. rend., 1905, 140, 506.

5 Constam and Bennet, Zeitsch. anorgan. Chem., 1900, 25, 265. 
gained a considerable vogue; and it has been found more satisfactory than hydrogen peroxide in the bleaching of human hair, since its alkaline nature enables it to remove grease and thus ensures a more uniform effect. In the bleach-works it is largely used; and it is generally employed here along with a catalyst such as the oxides of manganese and lead.

The constitution of sodium perborate is still a matter of doubt. Four formulæ may be suggested-

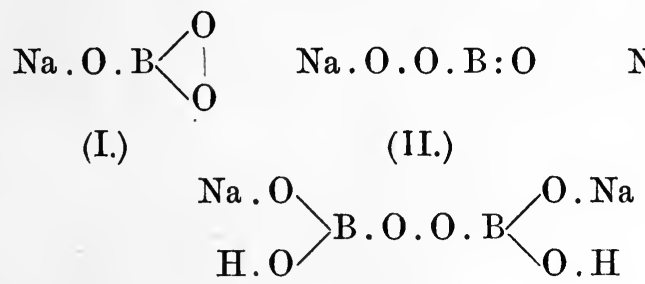

$\mathrm{NaBO}_{2}, \mathrm{H}_{2} \mathrm{O}_{2}$

(IV.)

Formula (III.) is evidently a meta-borate with hydrogen peroxide of crystallization; and it should, by analogy with other similar compounds, lose hydrogen peroxide on heating. In actual practice, however, when sodium perborate is heated to $60^{\circ} \mathrm{C}$. under diminished pressure, water is lost but no hydrogen peroxide is found in the distillate. ${ }^{1}$ Also, ammonium perborate exists as $\mathrm{NH}_{4} \mathrm{BO}_{3}$, which throws doubt on the presence of hydrogen peroxide in the sodium salt. Formula (IV.) is also excluded; as a compound of this type should give rise to a salt $\mathrm{Na}_{4} \mathrm{~B}_{2} \mathrm{O}_{6}$ on treatment with excess of alkali. No such reaction is known.

This leaves us with the choice between (I.) and (II.) In favour of the second formula we have an argument by analogy from other per-salts the constitution of which is known. The first formula is supported by the fact that a stable potassium compound is known which has the composition $\mathrm{KBO}_{4}, \mathrm{H}_{2} \mathrm{O}$ and which may be regarded as a salt of potassium hydroperoxide with perboric acid. This substance is remarkably stable and gives off only traces of oxygen when dissolved in water. This behaviour is not remarkable if perboric acid has the formula (I.); but if perboric acid is constituted as in (II.) the potassium salt would contain a chain of three oxygen atoms,

1. Bosshard and Zwicky, Zeitsch. angew. Chem., 1912, 25, 993. 
which, according to our normal views, would be unstable. The two possible formulæ of the potassium salt are shown below-

$$
\text { K.0.0.B }>_{0}^{O}
$$

Derived from perboric acid (I.). Derived from perboric acid (II.).

The reader must decide for himself which formulation he prefers. 


\section{CHAPTER V}

\section{ACTIVE NITROGEN}

\section{The Production of Active Nitrogen.}

THE fact that some of the elements can exist in more than one form has been common knowledge for years; but it is only recently that nitrogen has been proved to be capable of existing in allotropic modifications; and the results obtained during the investigation are of considerable interest since they display nitrogen in the guise of a very reactive element.

When electric discharges are passed through gases under low pressure it is sometimes found that the gases continue to glow for a time after the discharge has been interrupted; and Strutt ${ }^{1}$ has proved that this effect-in the case of air-is due to the mutual reaction of nitric oxide and ozone which are formed during the discharge. Similar phosphorescent combustions were observed in ozone when sulphur, sulphuretted hydrogen, acetylene, or iodine is present. $^{2}$ Pure nitrogen gives no after-glow when the simple induction coil discharge is employed $;^{2}$ but if a jar discharge and a spark-gap be utilized the glow is observed as in the other cases.

The practical details of the apparatus for producing active nitrogen are simple in the extreme. ${ }^{3}$ Carefully purified nitrogen from a gas-holder is drawn in a slow stream into a tube in which two electrodes are placed. The discharge between the electrodes activates the nitrogen, and the gas is then drawn on by the flow into another part of the tube, at which point it can be observed and its properties ascertained

1 Strutt, Phys. Soc. Proc., 1910, 23, 66.

2 Ibid., 1910, 23, 147. 303.

${ }^{3}$ Ibid. ; Proc. Roy. Soc., 1911, A, 85, 219 ; 1913, A, 88, 539 ; 1915, A, 91, 
without any interference from the electric discharge. The flow of gas is maintained by means of a mechanical pump.

When it is desired to test the effect of active nitrogen upon any other substance, the latter is placed in the observation part of the tube so that the stream of active nitrogen flows over it in passage.

\section{The Properties of Active Nitrogen.}

(a) Action on Non-metals.-When active nitrogen comes in contáct with the vapour of yellow phosphorus, some reaction occurs; for the nature of the glow changes and red phosphorus is deposited as a film upon the surface of the tube. ${ }^{1}$ Closer investigation revealed the fact that the reaction is not immediate; the nitrogen reacts with the phosphorus mainly after the glow has died down. ${ }^{2}$ Sulphur reacts with a blue flame or glow ; and a green material is deposited upon the glass. ${ }^{1}$ Apparently this is mainly nitrogen sulphide. ${ }^{2}$ Selenium is unacted upon. ${ }^{1}$ Arsenic shows a green glow during the reaction $^{1}$ and yields arsenic nitride. ${ }^{2}$ Antimony, carbon, and hydrogen gave no results. ${ }^{1}$ Oxygen destroys the glow of the nitrogen without producing any other luminosity. ${ }^{1}$ Iodine exhibits a very marked light blue flame when its vapour mingles with active nitrogen.

(b) Action on Metals.-When glowing nitrogen is passed over a pellet of metallic sodium heated a little above its melting-point, the sodium spectrum is shown brilliantly; and similar results were observed with other metallic elements. The phenomenon is due to the metal burning in the active nitrogen to form the nitride $;^{3}$ and the spectrum is simply the flame spectrum of the metal. ${ }^{4}$ Similar results were observed with zinc, cadmium, mercury, potassium, magnesium, and lead.1, 2 In the case of mercury an explosive compound was produced. 1

(c) Action on Compounds. - When ammonia is mixed with active nitrogen the glow is extinguished, but no chemical

' Strutt, Proc. Roy. Soc., 1911, A, 85, 219.

2 Ibid., 1913, A, 88, 539.

3 Ibid., 1912, A, 88, 539.

${ }_{4}^{4}$ Ibid., 1911, A, 85, 219; Trans., 1918, 113, 200. 
effects have been observed. ${ }^{1}$ Nitric oxide acts in a fashion quite different from what might have been anticipated. A greenish-yellow flame is observed; and nitrogen peroxide is produced. $^{2}$ Why active nitrogen should convert nitric oxide into the more highly oxygenated derivative nitrogen peroxide is not yet understood. Copper oxide destroys the glow of active nitrogen abruptly; but no chemical change was traceable; and it appears probable that the action is a catalytic one. ${ }^{3}$

Various salts were examined; but the observations are limited to their effects upon the glow of the active nitrogen. ${ }^{3,4}$ Stannic chloride and titanium tetrachloride give solid products containing nitrogen.

Turning to the action of allotropic nitrogen upon the carbon compounds, a considerable amount of data has been accumulated.1, 2, 3 Acetylene yields cyanogen; and a similar result was observed in the cases of ether, ethyl iodide, carbon tetrachloride, chloroform, bromoform, ethylene chloride, and ethylidene chloride. Benzene is much less reactive than acetylene, as might have been expected. Carbon disulphide produces a blue polymeric nitrogen sulphide and a polymeric carbon monosulphide. (Sulphur chloride yields ordinary yellow nitrogen sulphide..$^{5}$ ) Methane, hexane, and heptane are attacked with the formation of hydrocyanic acid.

(d) Spectrum.-The spectrum of active nitrogen is different from any other nitrogen spectrum. It contains in the visible a green, a yellow, and a red band. ${ }^{6}$

(e) Electrical Properties.-Active nitrogen has a marked conductivity. Apparently a current of the gas displays a behaviour from the electrical standpoint which brings it into line with a salted Bunsen flame.7

\section{The Influence of Impurities.}

At one time doubt was thrown upon Strutt's statement that pure nitrogen could give rise to the active form; and it

1 Strutt, Proc. Roy. Soc., 1911, A, 85, 219.

3 Ibid., Proc. Roy. Soc., 1911, A, 85, 219.

s Ibid., 1913, A, 88, 539.

- Ibid., 1911, A, 85, 219 ; Fowler and Strutt, ibid., 377 ; Strutt and Fowler, ibid., 1911, A, 86, 105.

'Tbid., Proc. Roy. Soc., 1911, A, 86, 56 ; ibid., 1912, A, 87, 179.

2 Ibid., 1911, A, 86, 56.

4 Ibid., 1915, A, 91, 303. 
was suggested ${ }^{1}$ that in order to obtain the phenomena described by him, a trace of oxygen was necessary. Strutt, however, showed that oxygen, instead of being advantageous, was actually deleterious, since the presence of 2 per cent. of it in nitrogen was sufficient to prevent the production of the glow. ${ }^{2}$ None the less, the controversy continued. ${ }^{3}$

Koenig and Elöd ${ }^{4}$ suggested that possibly the other investigators were at cross purposes in the matter owing to a lack of distinguishing between two different phenomena: the Strutt glow on the one hand and the chemical effects on the other. It seemed possible that these two effects were not necessarily co-existent; and since Tiede and Domcke used the presence of the glow as a test for active nitrogen, it seemed possible that misunderstandings had arisen in this way.

The main question was settled by Tiede and Domcke bringing their apparatus over to London so that the two processes could be tried side by side. The contradictory results were proved to be due to differences in the apparatuses; and it was found that active nitrogen can be produced even when no oxygen is present. On the other hand, minute traces of oxygen appear to have a favourable effect upon the process..$^{5,6}$

The discovery of this catalytic effect of oxygen led Strutt ${ }^{6}$ to a study of the influence of other substances upon the production of active nitrogen; and he established the fact that minute admixtures of other materials are capable of performing the same function as oxygen itself. The following substances all gave results, the most effective ones being placed first. Hydrogen sulphide, water, carbon dioxide, carbon monoxide, the three hydrocarbons, acetylene, ethylene, and methane, oxygen, mercury, chlorine, hydrogen, and the two inert gases, argon and helium. It was established that although the purest nitrogen will yield active nitrogen, yet in order to obtain the active form in any abundance it is necessary to have about $0 \cdot 1$ per cent. of some foreign gas present. ${ }^{7}$ Strutt

1 Comte, Physikal. Zeitsch., 1913, 14, 74 ; Tiede, Ber., 1913, 46, 340.

2 Strutt, Physikal. Zeitsch., 1913, 14, 215 ; cf. Koenig and Elöd, ibid., 165.

3 Tiede and Domcke, Ber., 1913, 46, 4095.

4 Koenig and Elöd, Ber., 1914, 47, 516.

5 Baker, Tiede, Strutt, and Domcke, Nature, 1914, 93, 478.

- Strutt, Proc. Roy. Soc., A, 1915, 91, 303.

? Cf. Strutt, Trans., 1918, 113, 200. 
suggests that the impurity acts by loading the electrons in the discharge and thus altering the character of their impact upon the nitrogen molecules.

\section{The Effect of External Conditions upon Active Nitrogen.}

The influence of temperature upon active nitrogen is very well marked. When the portion of the observation tube through which the gas passes is heated, the glow of the nitrogen is locally extinguished and only revives when the gas has gone further into a cooler part of the tube. From this, Strutt deduced that the change which produces the after-glow is accelerated by heating and retarded by cooling. ${ }^{1}$

Further investigation, however, threw doubt upon this view. ${ }^{2}$ When a specimen of active nitrogen was sealed up in a bulb and excited by an electrodeless discharge, it was found that at ordinary temperatures the glow lasted for about a minute. A similar experiment carried out with the bulb cooled to liquid air temperature proved that the glow was extinguished in fifteen seconds. Whilst the bulb was under the surface of the liquid air, the glow was very brilliant.

Curiously enough, when the bulb was plunged in boiling water the duration of the glow was also shortened.

Strutt then suggested that there were two factors to be taken into account. One of these was the direct temperature effect on the glow-transformation; the other was the destructive effect of the walls of the vessel, which had a catalytic action upon the phenomenon. The destructive effect would be increased by rise in temperature.

On this hypothesis, the results are interpreted as follows: When cooled to $-180^{\circ} \mathrm{C}$. the catalytic action of the glass is somewhat checked; but, in compensation, the glow-transformation in the gas occurs more rapidly at low temperatures and the glow is given out with greater intensity during a briefer period. On the other hand, when the bulb is heated too $100^{\circ} \mathrm{C}$. the catalytic effect of the glass is increased and its influence overbears the retardation of the transformation due to the higher temperature.

1 Strutt, Proc. Roy. Soc., 1911, A, 85, 219.

2 Ibid., 1911, A, 86, 262. 
If this hypothesis be correct, the transformation of active nitrogen is exothermic, but it is also unique among known reactions in that it proceeds more rapidly at low temperatures than at high ones. It must be emphasized that this is not yet more than a hypothesis ; but if it should prove correct it will have a considerable effect upon our ideas of reaction-conditions.

Turning to another influence which might be supposed to be capable of affecting active nitrogen, it has been shown by Strutt ${ }^{1}$ that an electric field has no action upon the properties of the gas. Neither the glow nor the chemical activity of the nitrogen seemed to be influenced.

\section{A Third Form of Nitrogen.}

Lowry $^{2}$ has detected the existence of nitrogen in a form which differs apparently both from ordinary nitrogen and from Strutt's active nitrogen. A current of air was passed through an ozonizer and then across a series of spark-gaps; and the gases were then examined in a spectroscope cell 64 feet in length, by means of which method the presence of minute traces of nitrogen peroxide could be detected.

It was found that when the ozonizer or the spark-gaps were used singly, no trace of nitrogen peroxide was detectable; but when the air from the ozonizer was passed over the spark-gap, the spectrum of nitrogen peroxide could readily be seen in the spectroscope.

From these results it appears that the ozonizer and the spark-gap taken separately do not activate the nitrogen of the air; but the successive action of them upon the stream of gas results in the formation of an active form of nitrogen which reacts with the ozone to produce nitrogen peroxide.

The active form of nitrogen thus produced is not identical with Strutt's variety, since, the latter is not oxidized by ozone.

1 Strutt, Proc. Roy. Soc., 1911, A, 85, 219 ; 1912, A, 89, 56.

2 Lowry, Trans., 1912, 101, 1152; Phil. Mag., 1914, 28, 412. 


\section{CHAPTER VI}

\section{ABSORPTION SPECTRA}

\section{General.}

THE physical properties of chemical compounds can be divided into two categories: colligative properties and non-colligative properties. The first of these groups comprises properties which are dependent purely upon the number of molecules present in the specimen we are examining; and of this type a good example is afforded by the pressure which a gas exerts at constant temperature. Non-colligative properties are in turn divisible into two sections: additive properties and constitutive properties. It is difficult to draw a hard-and-fast line between these two sections, for most properties are partly additive and partly constitutive. When we speak of an additive property, we usually mean one in which the number of atoms of each element in the molecule is the preponderating factor; while we regard as constitutive properties those in which the additive properties are masked by the greater influence of the mode of linkage of atoms in the molecule.

The study of additive properties is a much more simple line of research than the examination of constitutive properties. For instance, refractive index appears to be an additive relation, and its laws are already marked out in their main outlines; but when we take dielectric constant-which is simply the refractive index for waves of infinite length-the constitutive factor overwhelms the additive one, and up to the present time no definite relation has been traced between structure and dielectric constant.

Of all the constitutive properties of chemical compounds, the most complex appear to be absorption spectra. ${ }^{1}$ A few

1 For a full account of absorption spectra, see Smiles' The Relation between Chemical Constitution and Physical Properties, 1910. 
rough rules, some of which, like Armstrong's quinonoid hypothesis, are either mere words or obviously incorrect, are all that we have to guide us at the present time in tracing a connection between what is called "colour" and the chemical constitution of compounds. Much time has been wasted in discussing the question of physiological colour, a phenomenon of very little scientific value, since it depends upon the crude perception of the human eye, which can be thrown out of gear by a dose of santonine, and which, at best, excludes more of the spectrum than it perceives. But even our finest instruments to-day include within their range only a portion of the complete electrical, thermal, and luminous spectrum of any substance.

When we look at the sun, we see what is called the visible solar spectrum, that is, white light. If we hold up between our eye and the sun a beaker containing ferric chloride solution, we shall see a yellow colour. Now, it is obvious that the ferric chloride solution has added nothing to the sun's light; so if it has produced a change in the light which reaches our eye, it is clear that the solution must have abstracted something from the solar spectrum. What actually happens is that some of the rays of the spectrum are absorbed in the solution, so that instead of the complete solar spectrum, we now see what is called the absorption spectrum of ferric chloride.

Suppose that instead of merely looking through the beaker, we place it in front of a spectroscope and pass the sun's light through the ferric chloride solution. By looking through the spectroscope we shall then find that, instead of the whole solar spectrum being transmitted, some of the lines are absent, having been absorbed by the ferric chloride. By comparison with the usual solar spectrum we could easily determine exactly which lines have been absorbed, and in this way we should have a more or less accurate idea of the absorption spectrum of ferric chloride. In practice, however, this method suffers from many drawbacks, and instead of it we use the following arrangement:-

In Fig. 1 the apparatus is represented diagrammatically. $\mathrm{E} \mathbf{E}$ are the electrodes of an arc light. $\mathbf{C}$ is a cell containing the solution whose absorption spectrum is to be measured. $\mathrm{S}$ is the slit of the spectroscope. $\mathrm{P}$ is the prism. $\mathrm{O}$ is a photographic camera, containing a plate, Q. The electrodes, 
E E, may be of any metal or alloy which gives a well distributed series of lines in its spectrum; an iron arc is sufficient for most purposes. The cell, C, must have quartz ends, through which the light of the arc may pass to the slit $S$. If glass ends were used, a very large proportion of the invisible ultraviolet part of the spectrum would be cut off; for though glass is transparent to the visible spectrum, it is almost opaque with respect to the ultra-violet region. The spectrum of the arc is thus thrown upon the plate $P$, and by means of a diaphragm placed before the slit it is reduced to a narrow band. With the usual form of photographic spectroscope, the band is about 5 inches long and a quarter of an inch wide.

Before we can utilize the spectroscope for studying absorption spectra, however, it is necessary to prepare what we may call a standard plate. That is to say, we must have a photograph of the pure arc spectrum, and be able to identify the lines upon it. Suppose we are working with an iron arc. We first photograph the spectrum of the arc pure and simple, removing the cell $\mathrm{C}$. This negative forms the basis of our standard plate. We then smear some salt, say lithium chloride, upon the arc, and take another photograph of the spectrum. In this new photograph we shall have not only the iron spectrum, but in addition to it the characteristic lines of the lithium spectrum. A comparison of the two negatives will enable us to detect the lithium lines. We now look up the tables of

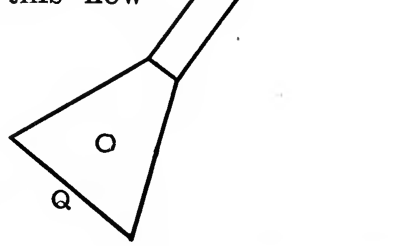

FIG. 1. the lithium spectrum, and select one or two of the chief lines whose wave-lengths have been accurately determined. These lines we must next pick out on the lithium-iron plate. Having found them, it is clear that we shall be able to find some characteristic lines in the iron spectrum close to them. We look up the position of these iron lines in the table of the iron spectrum, and thus find the exact wave-length in that 
part of the spectrum. It is obvious that here we have a double control : the lithium spectrum and the iron spectrum. All that now remains to be done is to note with a mapping pen on the margin of the standard plate the exact wave-lengths of the lines in the iron spectrum whose position we have thus established. Other salts are smeared upon the arc, and a series of photographs containing iron spectra interspersed with characteristic lines of the spectra of other elements, is thus obtained. In this way we obtain the bearings, so to speak, of a series of points in the iron spectrum, and the intermediate points are filled in by interpolation.

Having now got our standard plate, we must proceed to apply it in mapping absorption spectra. We fill up the cell $\mathrm{C}$, and adjust it so that the light of the iron arc passes through a certain thickness, e.g. $80 \mathrm{~mm}$., of a tenth-normal solution of, say, quinone in alcohol. We give an exposure of, say, fifteen seconds, and then develop the plate. On examination, the negative will be found to contain only part of the iron spectrum ; for the quinone solution will have absorbed a portion of the rays. - We lay the negative side by side with the standard .plate, and are thus enabled to read off the wavelength at which the absorption begins.*

Instead of using wave-lengths, it is more usual to reckon in frequencies - that is, the reciprocals of the wave-lengths.

It is clear that the absorption spectrum which we obtain in this way is conditioned by three things: the length of the exposure; the strength of the solution; and the thickness of the layer through which we photograph. Taking the case of a tenth-normal solution of quinone as an example, we shall find the following: If we begin with a layer of solution $80 \mathrm{~mm}$. in thickness, we find that all the spectrum beyond a frequency of about 2000 is absorbed (see Fig. 2, No. 1). When we reduce the thickness of the layer of solution in the cell to $70 \mathrm{~mm}$., a somewhat peculiar phenomenon occurs. The part of the spectrum to the left of 2000 comes through as

* The best modern spectrographs are provided with a scale of wave-lengths which can be photographed directly on the plate, side by side with the spectrum. In this way the frequent use of a standard plate is rendered unnecessary in actual practice; though the scale must be checked by means of a standard plate at intervals to make certain that it has not got out of adjustment. 
before, but in addition to it a small portion of light is transmitted at a frequency of about 2800. This is shown in Fig. 2, No. 2. When we reduce the thickness of the layer in the cell to $60 \mathrm{~mm}$., we find that this band of light transmitted at 2800 is a little broader, and it continues to increase in breadth as

$\begin{array}{ccccc}\text { Red end of spectrum } & & \text { Ultra-vlolet end of spectrum } \\ 1600 & 2000 & 2400 & 2800 & 3200 . . . \text { frequencies }\end{array}$

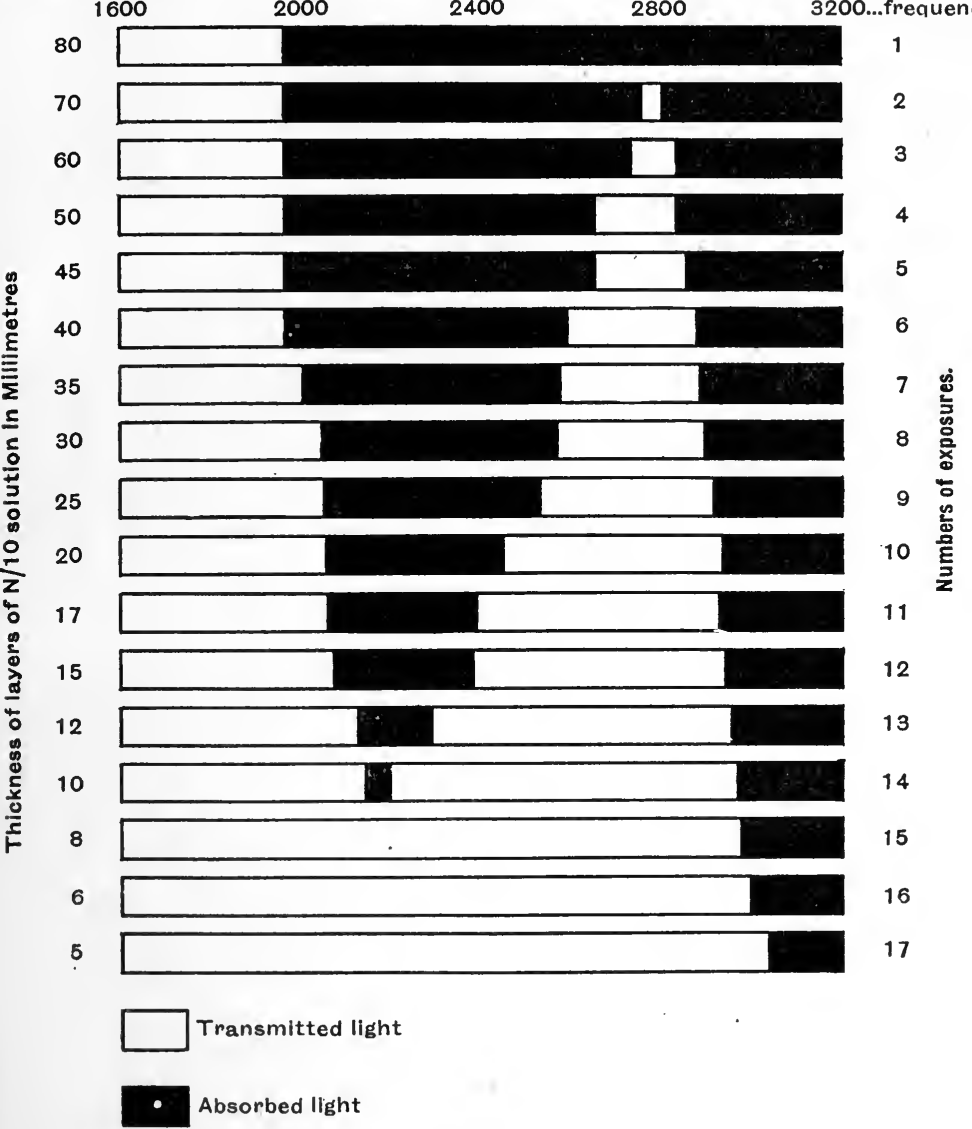

Fig. 2.

we reduce the thickness of solution through which the light is passed. In the end (see Nos. 14 and 15) the light absorption between 2000 and 2800 becomes reduced to a minimum and finally disappears, so that we have complete transmission. The rough triangle of absorbed light extending from No. 2 to 
No. 14 in Fig. 2 is called an absorption band. It will be observed that this band has a portion of transmitted light on either side of it. When no second portion of the light is transmitted, as in No. 1 or below No. 14, we have what is called general absorption.* The extreme apex of the absorption band which is shown in No. 14 is called the head of the band. The raggedness of the line running down from the frequency 2000 is due to experimental error, and is brought about by the fact that the iron spectrum is not quite uniform. $\dagger$ Some of the lines are strong, and others are weak, so that the readings are influenced to some extent. A group of strong lines may be transmitted where the weaker ones would not appear, so that in some cases our readings are a little too high, in other cases a little too low.

If, instead of using an $80 \mathrm{~mm}$. layer of a tenth-normal solution of quinone, we repeated the experiment with an $8 \mathrm{~mm}$. layer of normal quinone solution, we should find the absorption exactly the same in both cases, since the light, in traversing the layer, passes in each case through the same number of molecules. This is an example of what is termed Beer's Law.

Hartley was the first to see the value of results such as we have described. He pointed out that in this way we could. obtain a map of the absorption spectrum of a compound which would serve to identify it completely, for each substance has its own particular type of absorption spectrum. Some give shallow bands, some deep, and some none at all, but only general absorption. Again, some substances have one band, some two, and others many.

It is clear that the diagram in Fig. 2 would be too clumsy a mode of reproducing the results obtained, and Hartley therefore devised what are called curves of molecular vibration. The ordinates in this system represent the thickness in millimetres of a given solution, say a tenth-normal one, and the frequencies are plotted as the abscissæ. Baly and Desch ${ }^{1}$

* When an absorption band is present, we have what is called selective absorption.

$\dagger$ Hartley has employed electrodes composed of an alloy of lead, tin, and cadmium; the spark spectrum produced with this is practically continuous, and thus the disadvantages of the iron spectrum are avoided.

1 Baly and Desch Trans., 1904, 85, 1029. 
suggested that instead of the thickness being plotted on the ordinates, it would be more advantageous from some points of view to use the logarithms of the numbers representing the thicknesses, and this is the method now generally employed. Fig. 3 shows the molecular vibration curve of quinone in alcoholic solution plotted in this manner. The data on which the curve is based are the same as those from which Fig. 2 was drawn. The head of the band lies near the intersection of the lines 40 and 2200 . There is one other term which it

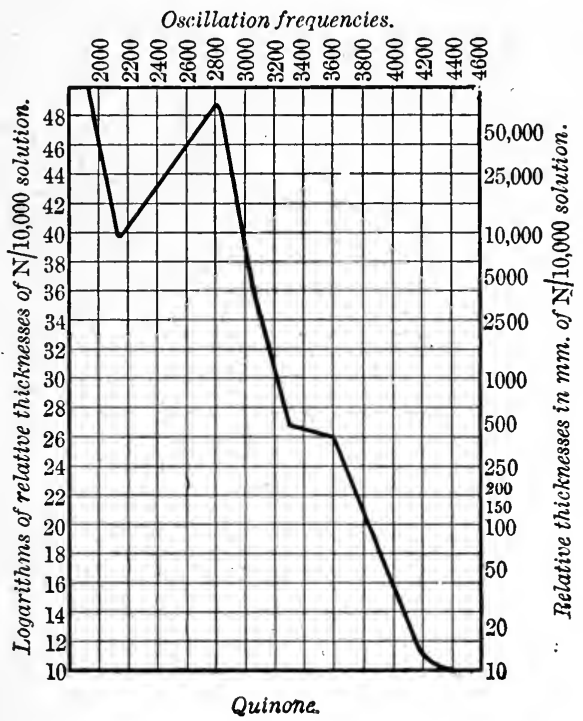

FIG. 3.

may be advisable to make clear before proceeding further, viz. the persistence of the band. By this term we mean the change of dilution over which the band remains in existence. An examination of the figure will show that the band comes into existence just above the line 48 and disappears just below the line 40 . It has therefore persisted over a change of dilution represented logarithmically by eight units.

In the following section we shall discuss the application of this method of investigation to the determination of the constitution of substances which cannot successfully be dealt with by purely chemical means. 


\section{The Hartley Method.}

Among the most interesting compounds in organic chemistry are those which are termed tautomeric (or desmotropic, pseudomeric, phasotropic, merotropic, or isodynamically isomeric). Whatever name be applied to these substances, their chief characteristic is that an apparently homogeneous mothersubstance can give rise to two isomeric derivatives according to the conditions under which the substitution reaction is carried out.

Now Hartley found that the replacement of hydrogen by a methyl or ethyl radicle produced no marked alteration in the selective absorption of substances, except in some special cases. In general, the members of a homologous series show a slight increase in general absorption as we go up the series, but in other respects there is very little change in the spectra. But if we take the spectra of two such substances as the alkyl derivatives of ortho-hydroxy-carbanil, whose constitutions are represented below, we shall find that we have hit upon a case in which the replacement of a hydrogen atom by an alkyl radicle has made a considerable difference in the absorption spectrum-
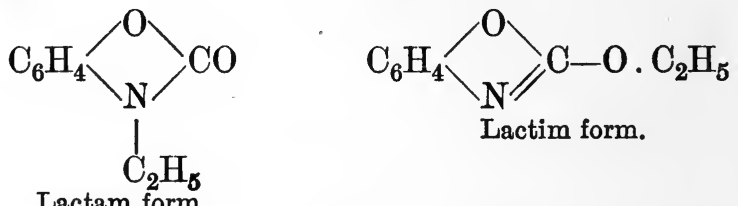

Lactam form.

The absorption spectra of hydroxy-carbanil and of the two ethyl derivatives (lactam and lactim forms) are shown in Fig. $4,{ }^{*}$ and it is evident on inspection that we have here two different types of absorption spectra. The spectrum of the lactam-compound differs completely from that of the lactimether. Now, when we compare the spectrum of the mothersubstance with those of the derivatives, it is obvious that it resembles the spectrum of the lactam-body rather than that of the lactim-ether. From this, Hartley deduces that the mother-substance must have a constitution resembling the

* The curves are reproduced from Hartley's work, and it should be noted that the ordinates represent thicknesses of solution, and not logarithms of thicknesses. 
lactam-form rather than one akin to the lactim-type. We should, therefore, ascribe to hydroxy-carbanil the constitution (I.) rather than (II.) -<smiles>[CH2][C@H]1NC(=O)O1</smiles>

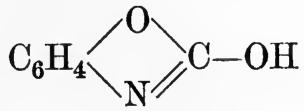

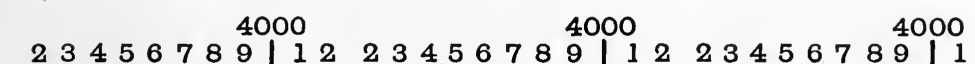

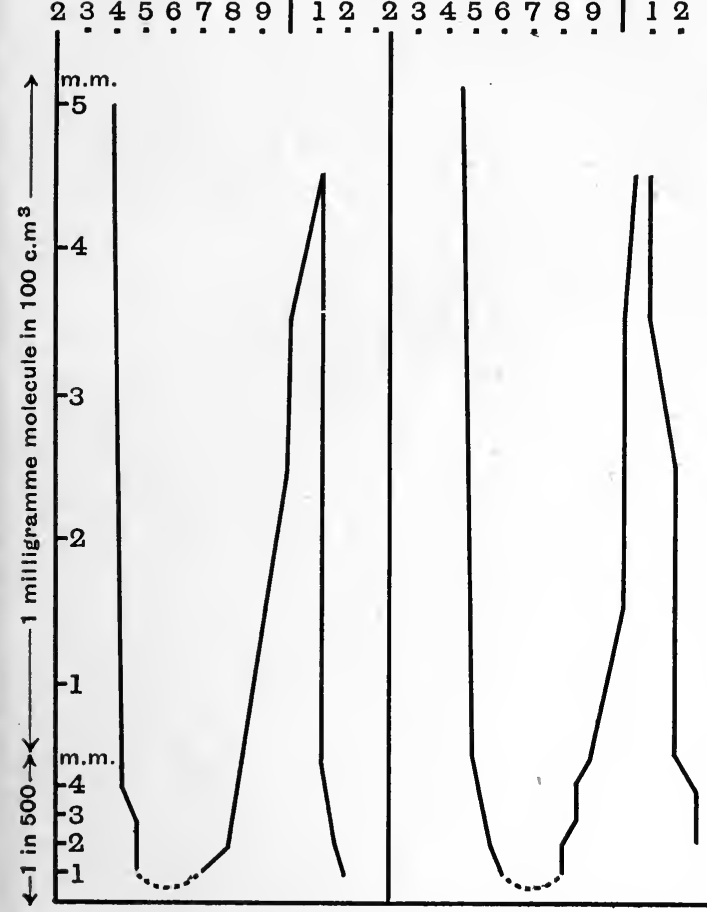

Lactam-ether
O-Hydroxy-carbanil Fig. 4.

This gives some idea of what is known as the Hartley method of determining the constitution of organic compounds. It has proved itself of great importance in many cases, and is certainly one of the most reliable in the solution of these problems.

\section{The Problem of General Absorption. .}

In recent years the study of absorption spectra has been for the most part confined to those questions which centre 
round the selective absorption of organic compounds, and it is not difficult to trace the causes which have led to this concentration of attention upon a particular section of the subject. The problem of the origin of colour in organic bodies attracted the attention of chemists both from the theoretical and, in the case of dye-stuffs, from the technical standpoint. Now, visible colour depends to a very considerable extent upon the presence of one or more definite absorption bands in the visible region; and when the work of Hartley had brought the investigation of the ultra-violet region iuto prominence, it was natural that the idea of colour should be enlarged to include bands in the invisible ultra-violet as well as the corresponding phenomenon in the visible region. And since in the visible region the hardest-fought controversy from the chemical point of view had centred round the presence or absence of absorption bands, it was only to be expected that similar discussions would arise as to the meaning of the numerous bands which were soon found to exist in the ultra-violet section of the spectrum. A second cause of the preference shown for the investigation of selective absorption is easy to detect. When we are dealing with substances which show absorption bands, a very slight alteration in their chemical structure is sufficient to produce a marked alteration in the character of the banded spectrum; and it is this delicacy which makes selective absorption so fascinating to those who delight in theorymaking. General absorption, on the other hand, is by no means so readily affected by minute constitutional changes. An examination of the curves on p. 67 will show that while the bands in the spectra of the lactam and lactim-ethers of hydroxy-carbanil are totally different in character, the general absorptions of the two substances differ but little from each other. Thus in the case of selective absorption we are dealing with a property which is susceptible to extremely minute influences; while in general absorption we have to do with forces which must differ markedly from each other before we can detect their effects.

When a compound is studied by the chemist there are seven chief questions which demand an answer:-

1. What elements does the molecule contain?

2. In what proportion are the various elements present? 
3. What is the number of the atoms in the molecule?

4. In what manner are the atoms linked together?

5. Is the substance saturated or unsaturated ?

6. How are the unsaturated centres of the molecule related to each other?

7. What is the arrangement in space of the atoms within the molecule?

Each of the factors indicated in the above questions enters into the problem of general absorptive power; and for the sake of convenience we may take them up in turn.

With regard to the influence of the various elements upon general absorption, it is impossible to lay down any hard-andfast rules. Saturated open-chain hydrocarbons are usually distinguished by being extremely diactinic; * and the introduction of halogen atoms into the molecule generally tends to increase the power of absorbing light. When we turn to other elements such as nitrogen, sulphur, or phosphorus, we complicate the problem by the introduction of unsaturation; so that it is hard to estimate the exact part played by the new element. The question is of considerable importance, however, and it is to be hoped that some investigator may find time to throw some light on the point.

From the point of view of spectroscopy, the second question is so closely connected with the first that no progress can be made in this direction until the first problem is in a much more advanced stage.

Turning now to the influence of the number of atoms in a molecule, we come to the question of homologous series. It has been found that if we examine the spectra of the methyl, ethyl, propyl, and butyl derivatives of a given substance, the power of general absorption increases as we go up the series. This was established in 1879 by Soret and Rillet ${ }^{1}$ in the case of the alkyl nitrates; and it appears to be a general property of homologous series. Each atom in a compound seems to exert a small amount of absorptive power, and by adding atom to atom we eventually obtain quite a marked effect on the spectrum.

We next come to the fourth point, the manner in which

- A substance of very low absorptive power is said to be diactinic.

1 Soret and Rillet, Compt. rend., 1879, 89, 747. 
the atoms are linked together within the molecule; and here, again, if we confine our attention to saturated substances the data are very sparse. Crymble, Stewart, and Wright ${ }^{1}$ have found that in the case of the alkyl bromides the normal substance has a much weaker power of absorption than the isomeric iso-form. For example, normal propyl bromide (I.) is much less absorptive than iso-propyl bromide (II.)-

\section{$\mathrm{CH}_{3} \cdot \mathrm{CH}_{2} \cdot \mathrm{CH}_{2} \cdot \mathrm{Br}$}

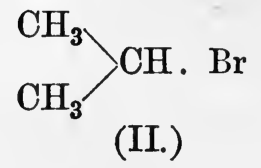

Results of this type might indicate that a symmetrically built molecule has more absorptive power than an isomeric molecule in which groups are unsymmetrically distributed.

When we take up the question of the influence of unsaturation upon the general absorptive power of molecules, the data are very full. ${ }^{2}$ It has been established, largely by the monumental researches of Hartley, that if we compare the spectra of a saturated and an unsaturated hydrocarbon, the unsaturated substance will greatly surpass the saturated one in its power of absorbing light. Since cyclic carbon chains are really unsaturated bodies, it might be expected that this class would show a higher absorptive power than open-chain bodies, and this has been found to hold good. With regard to the influence of the triple bond upon absorption there are very few data available; but it appears ${ }^{3}$ that its effect is not so strong as that of the ethylenic linkage.

We must now pass to the question of the effect produced by the introduction into the molecule of more than one unsaturated group; and at this point we reach a branch of the subject in which chemical constitution plays a very important part.

One of the best known characteristics of substances containing ethylenic bonds is their faculty of uniting with one molecule of bromine to form a dibromo-derivative, thus-

1 Crymble, Stewart, and Wright, unpublished observation.

2 Hartley, Brit. Assoc. Report, 1903.

3 Stewart, Trans., 1907, 91, 199; Ley and Engelhardt, Zeit. physikal. Chem., 1911, 74, 311. Compare Macbeth and Stewart, Trans., 1917, 111, 829. 
$\mathrm{CH}_{3}-\mathrm{CH}=\mathrm{CH}-\mathrm{CH}_{3}$

$\mathrm{Br}-\mathrm{Br}$
$\mathrm{CH}_{3}-\mathrm{CH}-\mathrm{CH}-\mathrm{CH}_{3}$

$\stackrel{\mathrm{Br}}{\mathrm{Br}}$

Let us now take the case of a substance containing two double bonds which are separated from one another by the interposition of one or more methylene groups. If to a gramme molecule of such a compound we add one gramme molecule of bromine, we shall find that the halogen is absorbed by one of the double bonds, while the second double bond remains intact, thus-

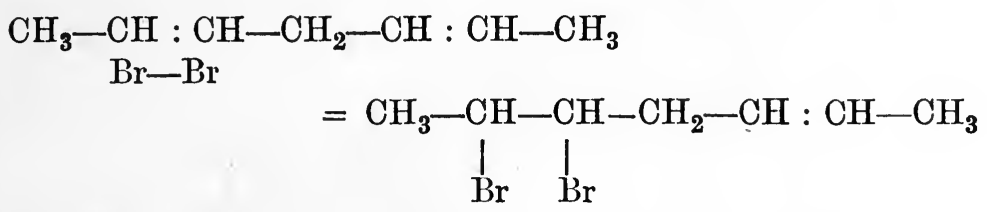

This is a perfectly normal behaviour; but if no methylene group be interposed between the two double bonds we obtain quite a different result, for instead of the halogen atoms attacking one of the double bonds they attack both simultaneously and a new double bond is formed as shown below-

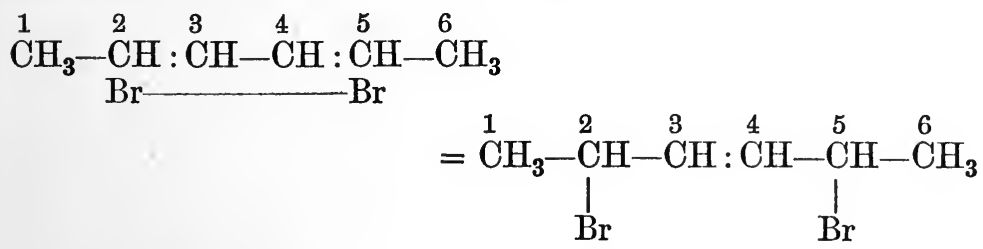

It will be seen from the formulæ that the original double bonds lay between the atoms 2,3 and 4,5 ; while in the final substance there is a new double bond in the 3, 4-position.

Thiele, ${ }^{1}$ from a study of many systems of this type, put forward his theory of "partial valencies." $\mathrm{He}$ assumes that in a double bond between two carbon atoms the whole of the available affinity of the atoms on each side of the double bond is not completely neutralized in the union, but that a certain amount remains free. This free affinity he terms a "partial valency," and he assumes that this partial valency serves to anchor any fresh atoms which come within range of the system.

1 Thiele, Annalen, 1899, 306, 87. 
Once anchored, the presence of the new atoms causes a rearrangement of affinity within the two molecules, with the result that the new atoms enter the system and reduce the double bond. If we represent the partial valencies by dotted lines, the steps in the process could be expressed by the formulæ below-

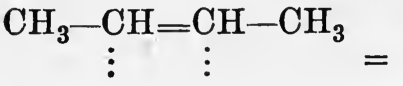

$$
\begin{aligned}
& \mathrm{Br}-\mathrm{Br} \\
& \begin{aligned}
\mathrm{CH}_{3}-\mathrm{CH} & =\mathrm{CH}-\mathrm{CH}_{3} \\
\vdots & \vdots \\
\mathrm{Br} & -\mathrm{Br}^{\mathrm{Br}} \\
= & \stackrel{\mathrm{CH}}{\mathrm{CH}_{3}}-\left.\right|_{\mathrm{Br}} ^{\mathrm{CH}}-\underset{\mathrm{Br}}{\mathrm{CH}}-\mathrm{CH}_{3}
\end{aligned}
\end{aligned}
$$

When we come to a system of the type-

$$
\mathrm{R}-\mathrm{CH}: \mathrm{CH}-\mathrm{CH}: \mathrm{CH}-\mathrm{R}
$$

we have what Thiele terms a system of "conjugated double bonds," and he explains the abnormal behaviour of this system in the following way: Since, under his postulates, the addition of new atoms to a system can take place only after they have been anchored by the partial valencies of the system, it is clear from experimental results that in a compound of the following type-

$$
\begin{array}{cccc}
\mathrm{CH}_{3}-\mathrm{CH}= & \mathrm{CH}-\mathrm{CH}=\mathrm{CH}-\mathrm{CH}_{3} \\
\vdots & \vdots & \vdots & \vdots \\
1 & 2 & 3 & 4
\end{array}
$$

only the partial valencies 1 and 4 are active. We are therefore driven to assume that the valencies 2 and 3, acting across the single bond, neutralize each other in some way, and are thus thrown out of action.* This state of affairs Thiele represents by the following scheme:-

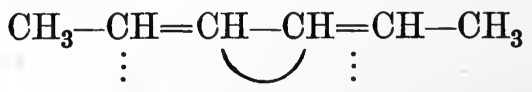

Such a grouping as this is anomalous not only from the chemical point of view but also in its influence upon the physical properties of compounds in which it occurs. ${ }^{1}$ Brühl $^{2}$ found

* The close agreement between this theory and actual experience is found in the case of benzene, where all the double bonds are conjugated in a single system with no free partial valencies. Hence, theoretically, benzene is not a true unsaturated body, and it is well known that experiment bears this out.

1 For a full discussion of this question, see Smiles' Relations between Chemical Constitution and some Physical Properties, 1910.

2 Brühl, Ber., 1907, 40, 878. 
that it had a marked effect upon refractive power, producing higher values than the calculated refractivities; Hilditch ${ }^{1}$ traced the same influence in the case of optical rotatory power; analogous results were observed by $\mathrm{Pascal}^{2}$ in diamagnetism; and Sir W. H. Perkin ${ }^{3}$ proved that similar effects could be detected in magnetic rotation.

Crymble, Stewart, Wright, and others ${ }^{4}$ have examined the spectra of numerous pairs of isomeric substances, one set containing isolated double bonds, while the isomeric bodies contained a conjugated grouping; and they find that in every case the substance which contains the conjugated system is much more absorptive than the isomeric body in which the double bonds are not so completely joined together in a single system. For example, citraconic acid contains three double bonds joined together in a single conjugated system; whereas in the isomeric itaconic acid one of the double bonds is separated from the rest by the interposition of the methylene group-

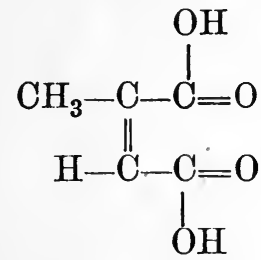

Citraconic acid.

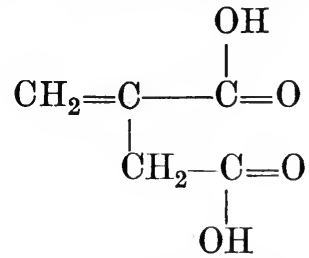

Itaconic acid.

Citraconic acid has a greater power of absorbing light than is shown by itaconic acid.

From this it is clear that when two unsaturated groups occur in a molecule in such positions that they are able to exercise a reciprocal influence upon each other, their joint effect upon the absorptive power of the substance is very much greater than when they are separated from each other by neutral atoms. One centre of residual affinity increases the absorptive power to a considerable extent as compared with a saturated compound ; two isolated centres in turn augment the compound's general

1 Hilditch, Trans., 1909, 95, 331, 1570, 1578; 1910, 97, 1091 ; Edminson and Hilditch, ibid., 1910, 97, 223.

2 Pascal, Compt. rend., 1909, 149, 342.

3 Sir W. H. Perkin, Trans, 1896, 69, 1141.

4 Crymble, Stewart, Wright, and Glendinning, Trans., 1911, 99, 451 ; Crymble, Stewart, Wright, and Miss Rea, ibid., 1911, 99, 1262. 
absorption; while a still greater power is produced by the co-ordination of the two centres into one system.

We must now pass to a consideration of the effect of stereoisomerism upon general absorption. The spectra of compounds containing asymmetric carbon atoms have been examined by Magini ${ }^{1}$ and by Stewart ${ }^{2}$ with the following results: Two optical antipodes have exactly similar absorption spectra ; mesotartaric acid has a greater power of general absorption than either active tartaric acid; while the spectrum of racemic acid shows a power of absorption greater than that of dextro-tartaric and less than that of meso-tartaric acid. Turning to the question of geometrically isomeric carbon compounds, the same authors have shown that maleic and citraconic acids possess much less light-absorbing power than the isomeric bodies fumaric and mesaconic acid-

Least absorbent.<smiles>O=C(O)/C=C\C(=O)O</smiles>

Maleic acid.

$\mathrm{CH}_{3}-\mathrm{C}-\mathrm{COOH}$<smiles>C=CC(=O)O</smiles>

Citraconic acid.
Most absorbent.

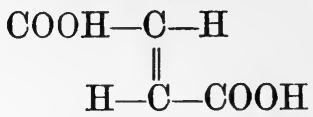

Fumaric acid.

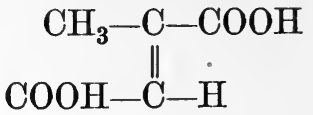

Mesaconic acid.

Hartley and Dobbie ${ }^{3}$ found that the spectra of the two stereoisomeric oximes of benzaldehyde were identical-<smiles>O/N=C/c1ccccc1</smiles>

Benzsynaldoxime.<smiles>O/N=C/c1ccccc1</smiles>

Benzantialdoxime.

At first sight it appears strange to find this difference in behaviour between the isomers containing an ethylenic bond and those which contain the linkage $-\mathrm{N}=\mathrm{C}=$, but a consideration of the formulæ of the various substances in the light of what has already been described in the preceding paragraphs will throw some light upon the point. We have already learned that general absorption is greatly influenced by the

1 Magini, J. Chim. phys., 1904,'2, 403.

2 Stewart, Trans., 1907, 91, 1537.

3 Hartley and Dobbie, Trans., 1900, 77, 509. 
presence in the molecule of some unsaturated centre; and that when we have two unsaturated centres in a substance, the absorptive power depends to a great extent upon their mutual interaction. Now the change from maleic to fumaric acid entails the shifting of one unsaturated carboxyl group with respect to the other; and the same is true of the change of citraconic into mesaconic acid: so that in both these cases we are to a great extent altering the interplay of forces in the molecule. Similar arguments hold.good in the case of the change of active tartaric acid into the meso-form, for here also the relative positions of the carboxyl groups are altered. When we come to the two oximes, however, the case is different, for there the two groups which change their relative positions in space are the phenyl and hydroxyl radicles. The phenyl group certainly possesses a marked degree of residual affinity; but the influence of the hydroxyl radicle on general absorption is very feeble, as is proved by the very low absorptive power of the aliphatic alcohols. Hence we might expect that these two groups would influence one another but slightly, and that a change in their relative positions would produce no marked difference in the absorptive power of the molecule. Support has been lent to this view by an examination of the absorption spectra of brassidic and erucic acids ${ }^{1}$ which were found to have almost identical powers of absorption-

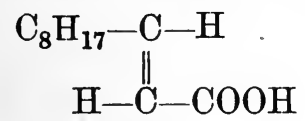

Erucic acid.

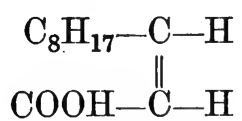

Brassidic acid.

It will be seen that in this case we have altered the relative positions of the groups $-\mathrm{COOH}$ and $-\mathrm{C}_{8} \mathrm{H}_{17}$; the first of these is an unsaturated centre, but the second is a saturated group, so that no great change in absorptive power is to be expected on the above hypothesis.

From the foregoing data it might be deduced that when the unsaturation of a molecule is arranged in a conjugated system, the greatest general absorptive power will be manifested when the free partial válencies are situated as far as possible from one another. In this connection, the cases of

1 Crymble, Stewart, Wright, and Arbuthnot, unpublished observation. 
dimethyl-diacetylene, hexatriene and benzene are not without interest. $^{1}$

$\mathrm{C}_{6} \mathrm{H}_{6}$ Dimethyl-diacetylene

$\mathrm{C}_{6} \mathrm{H}_{8}$ Hexatriene

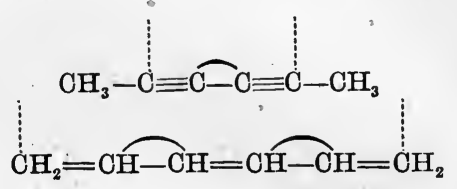

$\mathrm{C}_{6} \mathrm{H}_{6}$ Benzene

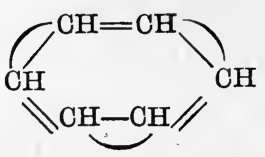

An examination of the molecular formulæ of these substances shows that hexatriene contains two hydrogen atoms more than either of the others; and by ordinary standards it should therefore be regarded as the most saturated of the three. On the other hand, its free partial valencies are further removed from one another than those of dimethyl-diacetylene can be; and benzene has no free partial valencies at all. If the suggestion put forward above be correct, hexatriene and dimethyl-diacetylene ought to show much greater absorptive power than benzene. When the spectra were compared, this was found to be the case.

\section{The Factors affecting Selective Absorption.}

Among organic compounds, it is found that the presence in the molecule of certain atomic groupings produces an absorption band in the spectrum of the substance. These particular atomic groupings are termed chromophores, ${ }^{2}$ and among them the most important are the following: $-\mathrm{CO}-,-\mathrm{N}: \mathrm{N}-$, $-\mathrm{N}: \mathrm{O},-\mathrm{C}_{6} \mathrm{H}_{5}$, and $=\mathrm{C}=\mathrm{NO} . \mathrm{OH}$.

An examination of the examples given above will show that all of them contain residual affinity; and if this residual affinity be saturated, the chromophoric power is lost. Thus the spectrum of acetone, $\mathrm{CH}_{3} \cdot \mathrm{CO} . \mathrm{CH}_{3}$, contains an absorption band; but when the carbonyl group is reduced it is found that the resulting isopropyl alcohol, $\mathrm{CH}_{3} \cdot \mathrm{CH}(\mathrm{OH}) \cdot \mathrm{CH}_{3}$, exhibits general absorption only.

1 Macbeth and Stewart, Trans., 1917, 111, 829.

2 Witt, Ber., 1876, 9, 522. 
When a chromophoric group is found in the structure of a molecule, the molecule is termed a chromogen. ${ }^{1}$ The nature of the chromogen sometimes influences the absorptive power of the chromophoric group which it contains; even in cases wherein the only unsaturation of the molecule is to be found in the chromophore. For example, the absorption band of camphorquinone is very much more persistent than that of diacetyl, ${ }^{2}$ although both compounds contain the double chromophore - $\mathrm{CO} . \mathrm{CO}-$ and the remainder of the molecule is saturated in each case-

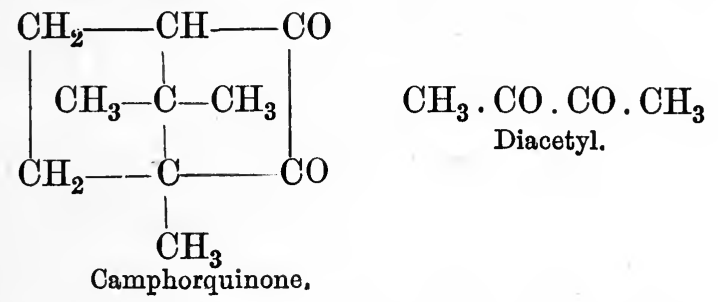

In general, however, the character of the absorption band shown by a substance depends mainly upon the chromophore, and is but little affected by the remainder of the chromogen when the latter contains no centres of residual aftinity.

The next question which presents itself is the influence exerted upon one another by two or more chromophores in the same molecule. This problem must be approached from two sides: for we may find the chromophores directly adjacent to one another in the molecular structure; or they may be separated from each other by saturated radicles.

Taking the first alternative in the case of the ketones, the following data throw some light upon the point :-

$\begin{array}{lll}\text { Acetone }: . \mathrm{CH}_{3} \cdot \mathrm{CO} \cdot \mathrm{CH}_{3} & \text { Absorption band in ultra-violet } \\ \text { Diacetyl }: \mathrm{CH}_{3} \cdot \mathrm{CO} \cdot \mathrm{CO} \cdot \mathrm{CH}_{3} & \text { Bright yellow } \\ \text { Triketopentane. } \mathrm{CH}_{3} \cdot \mathrm{CO} \cdot \mathrm{CO} \cdot \mathrm{CO} \cdot \mathrm{CH}_{3} & \text { Orange }\end{array}$

From this it will be seen that the accumulation of chromophores in adjacent positions in the molecule tends to bring the absorption band more and more towards the red end of the spectrum. It will be convenient to apply the name "conjugated chromophores" to such directly united chromophoric groups.

1 Witt, Ber., 1876, 9, 522.

2 Stewart and Baly, Trans., 1906, 89, 496, 503. 
Turning to the second type, wherein the two chromophores are separated from one another by a saturated grouping, we find quite different results. Acetone, $\mathrm{CH}_{3} \cdot \mathrm{CO} . \mathrm{CH}_{3}$, and acetylacetone, ${ }^{1} \mathrm{CH}_{3} \cdot \mathrm{CO} . \mathrm{CH}_{2} \cdot \mathrm{CO} . \mathrm{CH}_{3}$, both have bands in the same position in the ultra-violet; but the acetone band is seen in concentrated solutions whereas the band of acetylacetone is only brought out when the solution is very highly diluted. Since there are fewer molecules of the ketone present in the weak solution than in the strong one, it seems reasonable to conclude that the process giving rise to the band in acetone is feebler than that which takes place in acetylacetone. It is probable, therefore, that when two identical chromophores are separated from one another by a methylene radicle they act independently; the band produced by them is exhibited at the same position in the spectrum as if either of them was acting alone; but the dilution at which the band makes its appearance is increased by the joint action of the two.

A very interesting example of both arrangements of chromophores is to be found in the case of some alkyl derivatives of iodine. ${ }^{2}$ In this case the chromophore is the iodine atom attached to the carbon. The compounds examined were-

One Chromophore.

Methyl iodide . $\mathrm{CH}_{3}$. I

Ethyl ". $\quad \mathrm{CH}_{3} \cdot \mathrm{CH}_{2}$. I
Two Chromophores.

Methylene iodide $\cdot \mathrm{CH}_{2} \cdot \mathrm{I}_{2}$ Ethylene "

Three Chromophores.

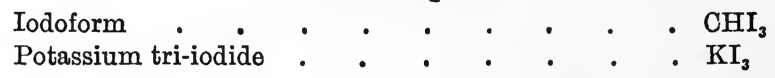

and their absorption spectra are shown in Fig. 5 .

Examination of the curve will show that ethyl iodide has an absorption band with its head at a frequency of 3900 and that this band occurs at the ordinate $3 \cdot 7$. Methyl iodide shows an almost exactly similar band, which has been omitted so as not to complicate the figure.

As can be seen from the figure, the spectrum of ethylene iodide contains a band whose head lies at 3900 , just as that of

1 Baly and Desch, Trans., 1905, 87, 766.

2 Crymble, Stewart, and Wright, Ber., 1910, 43, 1183. 
the mono-iodo-derivative does. The main difference between the two curves (ethyl iodide and ethylene iodide) lies in the fact that in order to obtain the ethyl iodide band a much thicker layer of solution must be used than is necessary in the case of ethylene iodide; for the one band occurs in the

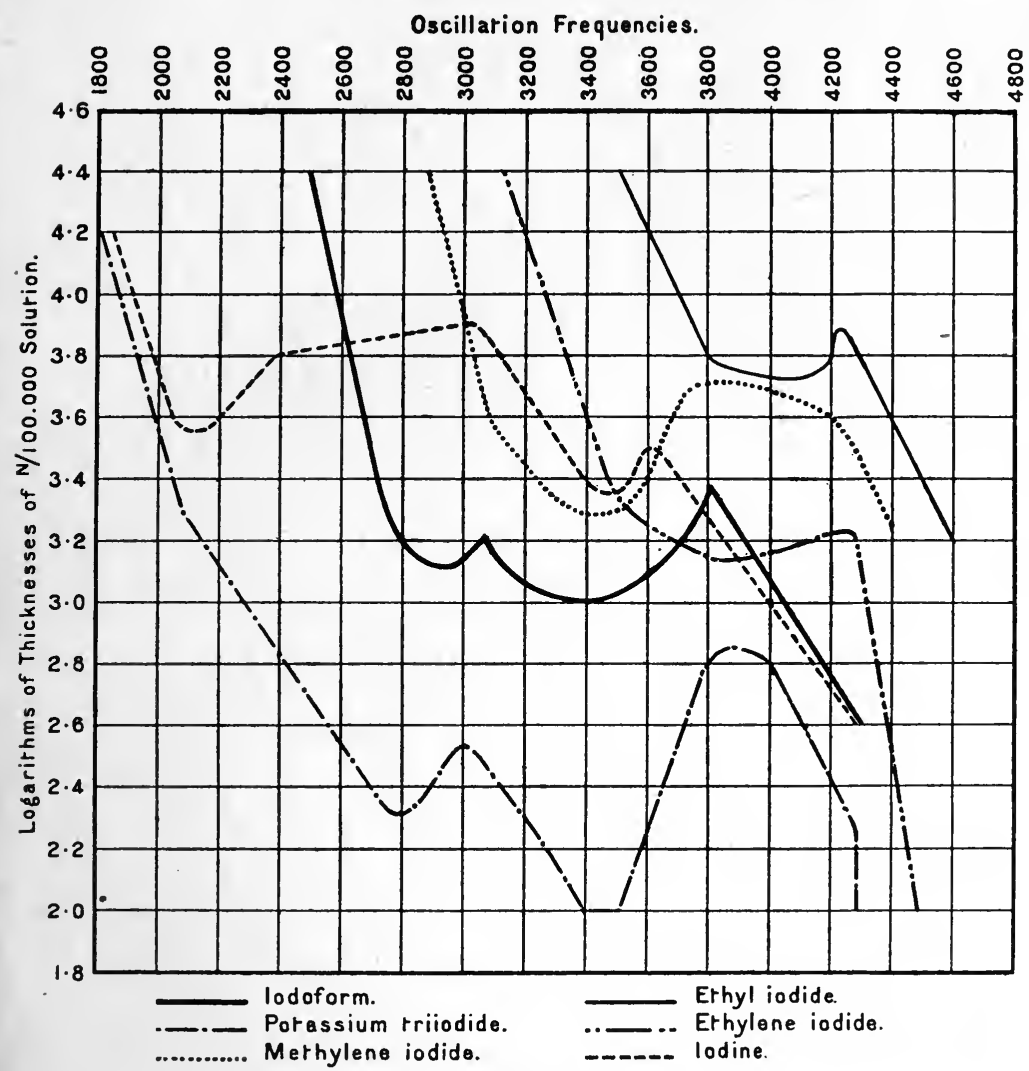

FIG. 5.

neighbourhood of the ordinate $3 \cdot 7$ whereas the other makes its appearance about ordinate $3 \cdot 1$ If we take a rough analogy from a piano, we might say that ethyl iodide and ethylene iodide both strike the same note; but ethyl iodide strikes it with the soft pedal down whereas ethylene iodide strikes it with the loud pedal in operation. In other words, the same process is going on in the two cases; but it is much more 
intense in the case of ethylene iodide owing to the fact that the band-producting group $\mathrm{R} \cdot \mathrm{CH}_{2}$. I occurs twice over in the same molecule.

Quite different results are found when the spectrum of methylene iodide is examined. Here there is no band at the frequency 3900 ; but instead a new band makes its appearance with its head at about 3400 -i.e. much nearer the red end of the spectrum. Evidently in this case we have to do with a conjugation of the two chromophores; and the band with its head at 3400 corresponds to the grouping: $\mathrm{CI}_{2}$.

Turning to the spectrum of iodoform, $\mathrm{CHI}_{3}$, it will be seen that this also contains a band with its head at 3400 ; and this must be ascribed to the presence of the grouping: $\mathrm{CI}_{2}$ in the iodoform molecule. But in addition to this band, another band makes its appearance with its head at 2900; and since no trace of this is to be found in the spectrum of $\mathrm{CH}_{2} \mathrm{I}_{2}$, its cause must be sought in the mutual influence of the three iodine atoms taken together.

An examination of the potassium tri-iodide curve on the figure bring out a further point of interest. The spectrum of potassium tri-iodide contains two bands whose heads lie at approximately the same frequencies as those of the bands in the iodoform spectrum. In the case of each compound we have three iodine atoms attached to a central atom; and this resemblance makes itself apparent in the absorption curves, notwithstanding the fact that the central atom in the one case is a carbon one and in the other case a potassium atom. This suggests that here also the influence of the non-chromophoric portion of the molecule has but little influence upon the chromophores.

We must now turn to yet another factor which may influence absorption spectra: the nature of the solvent. There seems to be no doubt that solvent action plays a considerable part; for when the spectra of substances in the form of pure vapour are compared with corresponding solutions in various solvents, it is found that the vapour spectra show a very large number of narrow absorption bands, whilst the solutions exhibit comparatively few bands of a much more diffuse character. In the case of aqueous solutions, ionization may produce different spectra at different concentrations; and 
even in non-aqueous solutions there may be interaction between the solute and solvent. ${ }^{1}$

\section{Valency and Absorptive Power.}

In the course of his study of the relations between the physical properties of elements and their places in the periodic system, Carnelley noted that under certain limitations the colour of a compound depends to some extent upon the atomic weights of the constituent elements. For instance, if we take compounds $\mathrm{AX}, \mathrm{BX}, \mathrm{CX}$, etc., where A, B, C, etc., represent successive elements belonging to the same sub-group in the periodic table, and $\mathrm{X}$ is any other element, then we shall find that, as we pass from the light element $A$ through the heavier element $B$ to the still heavier element $C$, the colours of the compounds deepen in tint. For example, let us take the case of the halogen compounds of cobalt and nickel-

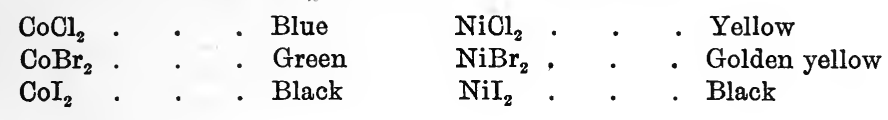

Here, as we pass from the chlorine atom, with an atomic weight of 35.5 , to the iodine atom, with an atomic weight of 127 , we find that the colour deepens from blue to black in the case of cobalt salts, and from yellow to black in the case of the corresponding nickel derivatives. Again, if we take the case of salts in which the acidic part remains constant throughout the series while the metal is replaced in rotation by the next higher member of the same sub-group, we get similar results.

\begin{tabular}{|c|c|}
\hline & \\
\hline & $\begin{array}{lll}\mathrm{NaI} & \cdot & \text { White } \\
\mathrm{YuI} & \cdot & \text { Cream coloured }\end{array}$ \\
\hline
\end{tabular}

This rule seems to be of fairly general application, but as it refers to substances in the solid condition, it is possible that the state of aggregation exercises a considerable influence. This might account for several of the exceptions to the rule which have been observed.

1 See, for example, the behaviour of iodine in alcohol, chloroform, and other solvents (Stewart and Wright, Ber., 1911, 44, 2819 ; Trans., 1917, 111, 183). 
Crymble ${ }^{1}$ approached the subject from a standpoint which evades this difficulty. On photographing the absorption spectra of potassium chloride and potassium sulphate, he found that 10 millimetres of a normal solution of either salt was diactinic. This proves, of course, that all the ions present in the solution under such conditions are diactinic. Hence it is clear that the colour of solutions of copper chloride or copper sulphate must be due to the copper ion. Taking as a standard a 10-millimetre thickness of a normal solution of a salt or a length of solution containing an equivalent amount of the metal, Crymble regarded a compound as "diactinic" when under these conditions light is transmitted up to a wavelength 2300 .

He found that under this definition metallic ions may be grouped into three classes-

A. Non-absorptive ions. This class includes the following metals :-

$$
\mathrm{Li}, \mathrm{Na}, \mathrm{K}, \mathrm{Be}, \mathrm{Mg}, \mathrm{Zn}, \mathrm{Cd}, \mathrm{Ba}, \mathrm{Al} \text {, and Th. }
$$

B. Ions showing general absorption only. In this class are-

$\mathrm{Hg}, \mathrm{Tl}, \mathrm{Sn}, \mathrm{Pb}, \mathrm{Sb}$, and $\mathrm{Bi}$.

C. Ions showing selective absorption. In this class may be placed-

$$
\mathrm{Cu}, \mathrm{Ce}, \mathrm{Mn}, \mathrm{Fe}, \mathrm{Au}, \mathrm{Cr}, \mathrm{U}, \mathrm{Co}, \mathrm{Pt}, \mathrm{Ti} \text {, and V. }
$$

Now, if we examine the metals placed in Class $A$, it is obvious that all of them possess a fixed valency: lithium, for example, forms only one chloride. The metals which show absorption (Class B and Class C), are all capable of exhibiting a change of valency : tin forms stannous and stannic chlorides, while copper forms cuprous and cupric salts. Thus it is established that metallic elements which are capable of exerting two degrees of valency have a much greater power of absorption than those metals which do not yield more than one chlorine compound.

A comparison of the majority of the elements in Class B and Class $\mathrm{C}$ will show that here also we can trace a general

1 Crymble, Proc., 1911, 27, 68. Lieutenant Crymble was killed in action in France in 1914. 
rule; for the elements in Class $\mathrm{C}$, whose ions show selective absorption, yield two series of salts in which the valency of the metal changes its valency by a single unit-

\section{$\mathrm{FeCl}_{2}$ and $\mathrm{FeCl}_{3} \quad \mathrm{CrCl}_{2}$ and $\mathrm{CrCl}_{3} \quad \mathrm{MnSO}_{4}$ and $\mathrm{Mn}_{2}\left(\mathrm{SO}_{4}\right)_{3}$}

whereas in the case of the metals in Class $\mathrm{B}$, which show general absorption only, the change of the "ous" form to the "ic" form entails a change of two units in the valency-

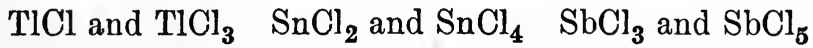

It is not yet certain whether this second generalization will hold good in every case; but the first rule put forward by Crymble marks a great advance in our knowledge of the subject.

If we apply to this problem of the metallic ions the results which we arrived at from our examination of the effect of unsaturation upon absorptive power in organic substances, we might be led to assume that the metallic atoms contain certain centres of residual affinity which are called into play by the combination of one atom with another. In the case of atoms such as sodium and potassium, which are capable of exhibiting one valency only, we might imagine that they contained only one such centre. In the case of atoms showing more than one valency, however, it seems possible that the atom may include two centres of residual affinity, which may act like two conjugated double bonds in an organic compound, and by their interplay may increase the absorptive power of the substance just as we found in the case of organic bodies.

\section{The Spectroscopic Determination of Chemical Change.}

Dobbie, Lauder, and Tinkler ${ }^{1}$ have shown how spectroscopic measurements may be used to throw light upon the extent to which chemical change takes place in a solution, and also upon the relative strengths of two reagents which have the same chemical effect. The substance used by them in this research was cotarnine, which is capable of existing in two forms. What the structures of these two forms are is of no importance to us at the present time; considerable controversy

1 Dobbie, Lauder, and Tinkler, Trans., 1904, 85, 121. 
has centred round the point. All that we need remember for our present purpose is that the change of one form into the other takes place under the influence of alkalis, while the reverse change is produced by acids.

In aqueous solution, cotarnine is yellow; that is to say, it has an absorption band in the visible region. Now, when the aqueous solution is treated with alkali, the yellow colour disappears, the amount of colour change being proportional to the quantity of alkali present. To compare the strengths of various alkalis, therefore, it is only necessary to add fixed quantities of them to a given solution of cotarnine, and estimate spectroscopically the change which they bring about in the substance.

In this way, Dobbie, Lauder, and Tinkler arrived at the results shown in the table below. The figures represent the percentages of the ammonium form of cotarnine which is converted into the carbinol form in presence of the corresponding amount of alkali.

\begin{tabular}{|c|c|c|c|c|c|c|}
\hline Strength of base. & $\mathrm{KOH}$. & $\mathrm{LiOH}$. & $\mathrm{NaOH}$. & $\mathrm{Ba}(\mathrm{OH})_{2}$. & $\mathrm{Ca}\left(\mathrm{OH}_{2}\right)$. & $\mathrm{NH}_{4} \mathrm{OH}$. \\
\hline $\mathrm{N} / 1$ & $98 \cdot 5$ & $98 \cdot 5$ & 98.5 & - & - & 45 \\
\hline $\mathrm{N} / 2$ & $97 \cdot 5$ & $97 \cdot 5$ & 97 & - & - & .40 \\
\hline $\mathrm{N}^{\prime} / 3$ & $96 \cdot 5$ & $96 \cdot 5$ & $95 \cdot 5$ & - & - & 30 \\
\hline $\mathrm{N} / 4$ & 95 & 94 & 94 & - & - & - \\
\hline $\mathrm{N} / 6$ & 93 & 93 & 93 & - & - & 25 \\
\hline$N^{\prime} / 7$ & 92.5 & - & 92.5 & - & - & - \\
\hline $\mathrm{N} / 8$ & 91 & 92.5 & 91 & 90 & - & - \\
\hline $\mathrm{N}^{\prime} / 10$ & 89 & 90 & 89 & - & - & 20 \\
\hline $\mathrm{N} / 20$ & 83 & $82 \cdot 5$ & $82 \cdot 5$ & - & - & - \\
\hline $\mathrm{N} / 50$ & $67 \cdot 5$ & 70 & 70 & $67 \cdot 5$ & $67 \cdot 5$ & 10 \\
\hline $\mathrm{N} / 100$ & $52 \%$ & $57 \cdot 5$ & $52 \cdot 5$ & 525 & 50 & - \\
\hline$N^{\prime} / 200$ & $42 \cdot 5$ & $42 \cdot 5$ & 40 & 40 & 40 & - \\
\hline $\mathrm{N} / 400$ & 25 & 20 & 20 & 20 & 20 & - \\
\hline $\mathrm{N} / 600$ & 20 & 20 & 20 & 20 & 20 & - \\
\hline
\end{tabular}

The possible applications of this method are, of course, almost inexhaustible; and it would be quite feasible to enlarge its scope and make it a means of measuring reaction velocities.

\section{The Spectrophotometer.}

Hitherto in this chapter we have confined our attention to the results which have been attained by the use of Hartley's curves of molecular vibration; but it is necessary to say a few 
words with regard to a newer method ${ }^{1}$ of measuring absorption spectra which has been brought into practice in comparatively recent times. This method yields results to which a definite physical meaning can be attached.

The object of the spectrophotometer is to measure quantitatively the proportion of light of a given wave-length which is absorbed when it passes through the substance under examination. Hartley's method, on the other hand, was a more or less qualitative one; for in it no attempt was made to estimate the proportion of light absorbed. In the Hartley method we merely measure the number of molecules of the substance which is required to reduce the light traversing them to such an extent that it will fail to impress a photographic plate after it has emerged from the substance; and it is obvious that this is a purely arbitrary standard to set up.

The simplest form of the spectrophotometer can be very briefly described. Between the ordinary quartz spectrograph and the light source is introduced the photometer proper. This consists of two revolving discs, one pierced with a fixed aperture, the other having an aperture which can be varied at will. Behind the fixed aperture is placed a cell containing a solution of the substance under examination. The light from the source travels in two rays, one of which passes through the variable aperture to the slit of the spectrograph whilst the other passes through the fixed aperture and the solution and thence to the slit. At the slit the two beams are received on the two halves of a biprism and are directed on to the photographic plate, where they are registered side by side.

A series of photographs is taken, the aperture of the disc being varied for each exposure. During the exposure, the discs are rotated at a uniform speed by means of a motor. On examining the plate, we find the photographs arranged in pairs. In one of each couple, the amount of light passing to the plate has been reduced by the variable aperture; in the case of the other the reduction in the amount of light has been produced by the absorption of the substance in the cell. If at

1 Bielecki and Henri, Compt. rend., 1912, 155, 456, 1617; 1913, 156, 550, $884,1322,1860 ; 157,372 ; 1914,158,567,866,1022$, 1114; Ber., 1913, 46, 2596,$3627 ; 1914,47,1690$. A full account of the work is to be found in Henri's Etudes de photochimie (1919). 
any part of the spectrum we find that the two photographs show equal darkening on the negative, it is clear that the intensity in the two cases has been equal. We know what proportion the aperture of the sector during this experiment bore to its full aperture; so that we know the proportion of light excluded from the plate in this instance. Since the same amount of light was obviously excluded by absorption in the cell-for the effects of the two beams on the plate are the same-it is clear that we can read off directly the proportion of light absorbed by the solution of the substance which we have used. For example, if we find that when the sector is only one-tenth open we get lines of equal blackness in the two spectra at frequency 3450 , we can say that for that particular frequency the substance absorbs nine-tenths of the light which enters the cell, and only allows one-tenth to pass through to the plate.*

Since photographic plates vary from batch to batch as regards their sensitiveness to different portions of the spectrum, it is necessary to calibrate the instrument carefully for each batch of plates used.

\section{The Calculation of Absorption Curves.}

V. Henri ${ }^{1}$ has shown that in certain cases it is possible to calculate the curve of absorption of substances, provided that certain fundamental data are known. In the first place, it is necessary to know what chromophoric groups the compound contains ; secondly, the relative position of these groups in the molecule must be ascertained; and thirdly, the frequencies of the infra-red absorption bands produced by these chromophoric groups must be known.

When these various factors have been ascertained, the absorption curve is calculated from the following scheme :-

$$
\nu=\nu_{0}-r \nu_{i} \quad \varepsilon=a \varepsilon
$$

where $\nu$ and $\varepsilon$ are the corresponding frequencies and absorptions of the compound under consideration, $\nu_{0}$ and $\varepsilon_{0}$ are the values of

* In actual practice the sector opening is calibrated accurately by photographing a substance of a known absorptive power and applying the necessary corrections from the data thus acquired.

1 V. Henri, Etudes de photochimie, chapter vi. 
frequency and absorption coefficient for the chromophore, $\nu_{i}$ is the frequency of the infra-red band of the chromophore, $r$ is an integer which rarely exceeds 5 and $a$ is a numerical factor.

To make the matter clear, a concrete case may be given. Suppose that it is desired to calculate the absorption curves of oxalic acid. The first step is to ascertain the absorption spectrum of acetic acid, which serves as a basis. In this particular example, the value of $\nu_{0}$ is $240 \times 10^{12}$ and $\alpha$ is 2, according to Henri's work. We select some particular frequency in the acetic acid spectrum and calculate the corresponding frequency in the oxalic acid spectrum by subtracting $240 \times 10^{12}$. To get the height of the ordinate corresponding to this abscissa in the oxalic acid spectrum we take the value of $\varepsilon$ at the original frequency in the acetic acid spectrum and multiply it by 2 ; and we then use this value as the ordinate in the oxalic acid spectrum we are calculating. Having thus ascertained the abscissa and ordinate for one point on the oxalic acid curve, we proceed to calculate the other points in an analogous manner until the curve is complete.

It will be seen that the calculation is a somewhat laborious one; but the results are in many cases wonderfully accuratethe calculated and observed curves practically coinciding with one another. Even in the case of such complex substances as uric acid (I.) it is possible to obtain a close approximation between the experimental and calculated curves when the latter is based on the spectrum of isobarbituric acid (II.)

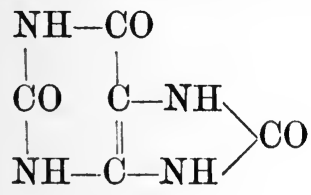

(I.)

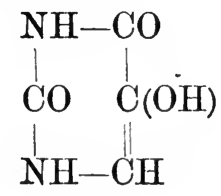

(II.)

There can be no doubt that the work of Henri represents almost as great a stride in advance as the investigations of Hartley did in their time. If absorption spectra can thus be brought within the group of "calculable" values, a new and extremely practical weapon will be placed in the hands of the synthetic dye industry; while on the purely scientific side, great advances will be possible. It is perhaps too soon to anticipate results in the near future, as Henri has pointed out 
the extremely laborious nature of the work entailed in ascertaining the fundamental constants; but the mere inception of the method is in itself a very important advance.

\section{Conclusion.}

In absorption spectra at the present time, one of the most promising fields for investigation appears to be the influence of solvents. If a long series of experiments were carried out in which attention was paid to the physical properties (such as dielectric constant) of inert solvents, one can hardly doubt that some important results would follow. Another branch of the same subject is the influence of the solvent upon reactionvelocities, and here it might be possible to detect a parallelism between the action of the solvent upon the reacting bodies and its action upon their spectra.

Again, spectroscopy furnishes us with a means of watching the changes which take place while a reaction is going on in a solution; and it-seems probable that interesting results might be acquired by a study of reactions which may or may not involve the formation of an unstable intermediate product, or of those which result in intramolecular change.

A field which appears to have been barely touched is the examination of analogous compounds of similar elements. For example, it might be of interest to discover whether there was any relation between the absorption spectra of an alkyl ether, a sulphide and a selenide and the chemical properties of the three substances. Parallel results might be found in the examination of derivatives of tri- and penta-valent nitrogen, phosphorus and arsenic. In the "onium" salts, also, the spectra might throw some light upon the cause of the ionization of the negative radicle.

At the present time we are ignorant of the cause of colour in many of the simplest substances. For example, if nitrogen and hydrogen, both colourless gases, be combined, we get a colourless gas, ammonia, formed. But if for hydrogen we substitute oxygen, we get a brown gas, $\mathrm{NO}_{2}$, which on polymerisation gives us a much more weakly coloured body, $\mathrm{N}_{2} \mathrm{O}_{4}$. It is clear that in cases such as these one atom has the effect 
of stimulating another into a certain state of vibration, while other atoms have not this power.

At the present day, the problem of what is termed residual affinity is rapidly being forced to the front in many widely different lines of investigation, and it is becoming more and more evident that our ordinary graphic formulæ are reaching the end of their usefulness. As an expression of the purely chemical behaviour of compounds they have played a wonderful part on the chemical stage, and in the future they will no doubt aid us in this as well as any substitute which can at present be imagined. But when we come to the relation between chemical constitution and physical properties the ordinary graphic formula, devised as it was to suit a totally different set of circumstances, cannot be blamed if it fails to throw much light on the question. What will be required in future is a standardization of what we describe as residual affinity, and it appears possible that spectroscopy may aid us in this line. Assuming the electronic basis of matter, we are driven to regard the chemical, electrical, and physical properties of a compound as different facets of a whole, or as interpretations of the electrons' motions in terms of various crude measurements with different units. At the present time our measurements of residual affinity are in the atomic stage, that is to say, we ascribe to both a compound containing an ethylenic bond and one containing a carbonyl group the same amount of residual affinity since they are both capable of combining with two hydrogen atoms. If it were possible to apply some spectroscopic measure of residual affinity, we should be in possession of a much finer measuring instrument, and it is not improbable that the differentiation between: one compound and another in this way would lead to valuable results. Ordinary chemical means can carry us only to the confines of the atomic world, but for a true understanding of the whole field we must be able to penetrate into the subatomic, or electronic universe, for it is in this region that the real changes occur which we measure in the ordinary chemical reaction. 


\section{CHAPTER VII}

\section{X-RAYS AND SOME ATOMIC PROPERTIES}

\section{The Phenomena of Crookes' Tubes.}

WHEN an electric current passes through a highly evacuated tube, three types of rays may be produced, according to the experimental conditions; cathode rays, positive rays, and $\mathrm{X}$ rays. As a parallel set of phenomena will be met with when we come to deal with the radioactive elements, it seems best to deal with all three classes here, though strictly the first two types lie outside the scope indicated by the title of the present chapter.

From the cathode of a Crookes' tube, cathode rays are thrown off at right angles to the surface. They are streams of electrons travelling at very high velocities in straight lines ; and are capable of penetrating thin sheets of aluminium or gold leaf. They ionize gases through which they pass; and produce an image on a photographic plate. When they impinge upon certain materials they set up fluorescence or phosphorescence; and they also heat up any substance by their impact. Finally, owing to their electrical nature, the particles which compose the stream can be deviated from their straight paths by magnetic or electric fields. Each electron represents a charge equal in quantity but opposite in sign to that carried by a hydrogen ion.

The second type of rays generated in a Crookes' tube is known by various names: canal rays, Kanalstrahlen, Goldstein rays or, in recent times, positive rays. They are streams of positively charged molecules of the residual gas in the tube which are produced when the cathode is pierced with one or more apertures; and they travel in straight lines in a direction opposite to that taken by the cathode rays. They have 
less power of penetrating matter than cathode rays have; and they are not so susceptible to deviation by electrical or magnetic fields.

Finally, we come to the X-rays, which are produced when cathode rays impinge upon matter. For long after their discovery, the nature of the $\mathrm{X}$-rays remained in doubt; but it is now known that they are vibrations similar to light, though their wave-lengths are extremely small as compared with those of light-waves. Like light, an X-ray can be diffracted, and polarized. When passed into a solid, an X-ray pencil is "scattered" just as light is scattered in a foggy atmosphere.

\section{Characteristic X-rays.}

When an X-ray beam impinges upon matter it may produce three types of radiation : scattered X-rays, characteristic X-rays, and corpuscular rays. The proportions in which these three vibrations are generated vary with the atomic weight of the element upon which the parent X-ray strikes. Elements of low atomic weight radiate mainly scattered $\mathrm{X}$-rays; an element of higher atomic weight, such as zinc, emits for the most part characteristic rays; whilst the heavy elements give off more corpuscular radiation than the lighter ones, mass for mass. The heavy elements scatter incident $\mathrm{X}$-rays to a greater degree than the lighter elements, when account is taken of their relative masses.

Scattered X-rays are of no great importance from the chemical point of view; and we shall deal in a later section with corpuscular radiation; so for the present we may confine our attention to the characteristic rays which are emitted by different elements when X-rays strike their surfaces.

An ordinary X-ray tube generates X-rays of many different qualities simultaneously; so that the beam arising from it is composed of a mixture of different vibrations just as white light is. If such a mixture of X-rays be allowed to fall upon a metallic surface, it is found that the metal is stimulated and gives off characteristic X-rays of definite wave-lengths. ${ }^{1}$ The nature of the new radiations depends solely upon the metal

1 Barkla and Sadler, Phil. Mag., 1908, 16, 550; Kaye, Phil. Trans., 1909, A, 209, 123. 
from which they are generated and has no relation to the properties of the original X-ray, provided always that the parent $\mathrm{X}$-ray is harder* than the characteristic radiation of the metal used. If the parent rays are too soft, no characteristic ray is excited by them. A rough rule is that the characteristic radiation of a given atom can excite the corresponding radiation of a lighter atom, but cannot produce any such effect in the case of a heavier atom.

An analogy to this phenomenon is to be found in the case of fluorescence excited by light. If we allow white light, which is a mixture of many wave-lengths, to fall upon a solution of quinine, we get a blue fluorescence produced which is characteristic of quinine. The same white light falling upon a fluorescein solution generates a green fluorescence, which is characteristic of fluorescein.

It has been shown that some elements give out several kinds of characteristic radiations, and these types are known as the $\mathrm{K}, \mathrm{L}$, etc., series. The $\mathrm{K}$ radiation is about three hundred times more penetrating than the $\mathrm{L}$ type. Both of these radiations conform to an empirical relation, known as Owen's Fifthpower Law, which connects quality of the radiation with the atomic weight of the metal emitting it. This law states that the penetrating power of the radiation is roughly proportional to the fifth power of the atomic weight of the metal from which the rays are generated. ${ }^{1}$

A simple relation has been established between the penetrating powers of the $K$ and $L$ radiations and the atomic weights of atoms. ${ }^{2}$ If an element of atomic weight $A_{L}$ emits a soft (L) radiation of a certain degree of hardness, then the atomic weight $A_{K}$ of a particular element having a $K$ radiation of the same hardness is given by-

$$
A_{K}=\frac{1}{2}\left(A_{L}-48\right)
$$

These characteristic $\mathrm{X}$-rays are governed entirely by atomic factors and are not affected by molecular conditions; for an element has been shown to give exactly the same quantity of

* The terms " hard" and "soft" applied to X-rays indicate the penetrative power of the rays, hard rays being more penetrating than soft ones. The hard rays are probably of shorter wave-length than the soft ones.

1 Owen, Proc. Roy. Soc., 1912, A, 86, 426.

2 Chapman, ibid., 439 
characteristic rays whether it exists in the free state or in combination as a salt. ${ }^{1}$ For example, $\mathrm{Fe}, \mathrm{Fe}_{2} \mathrm{O}_{3}, \mathrm{Fe}_{3} \mathrm{O}_{4}, \mathrm{FeSO}_{4}$, and $\mathrm{K}_{4} \mathrm{Fe}(\mathrm{CN})_{6}$ all emitted the characteristic X-rays of iron. ${ }^{2}$

It has been found that strong characteristic radiations may be obtained in the following manner. ${ }^{1}$ The cathode stream of a Crookes' tube is allowed to fall upon an anti-cathode of the metal whose characteristic radiation it is desired to produce. Screens of the same metal are then placed in the path of the $\mathrm{X}$-rays generated at the anti-cathode; and in this way any non-characteristic radiations are converted into those characteristic of the metal under examination. Whiddington ${ }^{3}$ determined the minimum velocity which the cathode particles must possess in order to excite the characteristic radiation; and this also proves to be a function of the atomic weight. If $V_{\mathbf{K}}$ is the minimum velocity of the cathode rays in $\mathrm{cms}$. per second; and $\mathrm{A}$ is the atomic weight of the metal composing the anticathode: then in the case of the $\mathrm{K}$ radiations the following relation holds :-

$$
\mathrm{V}_{\mathrm{K}}=\mathrm{A} \cdot 10^{8}
$$

This has been tested for a range of elements extending from aluminum to selenium.

The transformation of the energy of cathode rays into that of X-rays has been shown by Beatty ${ }^{4}$ to be influenced by the atomic weight of the material of the anti-cathode. The following relation appears to hold:-

$$
\frac{\mathrm{X} \text {-ray energy }}{\text { Cathode ray energy }}=2.54 \times 10^{-4} \mathrm{~A} \beta^{2}
$$

where $\mathbf{A}$ is the atomic weight and $\beta$ is the velocity of the cathode rays expressed as a fraction of the velocity of light $\left(3 \times 10^{10} \mathrm{cms}\right.$. per second $)$.

For example, if a platinum anti-cathode be excited by cathode rays with a speed of $10^{10} \mathrm{cms}$. per second, we have $A=195$ and $\beta=\frac{1}{3}$ so the fraction becomes equal to $6 \cdot 61 \times$ $10^{-3}$. These results refer only to primary $X$-rays; and if, in 446.

1 Kaje, Phil. Trans., 1908, A, 209, 123; Chapman, Phil. Mag., 1911, 21,

2 Glasson, Proc. Camb. Phil. Soc., 1910, 15, 437.

3 Whiddington, Proc. Roy. Soc., 1911, A, 85, 323.

4 Beatty, Proc. Roy. Soc., 1913, A, 89, 314. 
addition, characteristic X-rays are excited, their effect must be added to that which is given by the formula above.

We must now turn to the question of the absorptive power which elements exhibit when penetrated by characteristic rays. ${ }^{1}$ The absorption coefficient $\lambda$ is derived from the expression: $\mathrm{I}=\mathrm{I}_{\mathrm{o}} e^{-\lambda x}$, wherein I represents the transmitted X-radiation, $\mathrm{I}$ 。 represents the incident $\mathrm{X}$-radiation, and $x$ stands for the thickness of the layer of material through which the X-rays pass. Since the density of the material is a factor in the problem, it is usual to take it into account and define the absorption by the fraction $\frac{\lambda}{\rho}$ where $\rho$ is the density of the substance under examination.

The absorption phenomena are rather complicated; and to make them clear it will be best to take a concrete example. Suppose that we use a nickel cathode as the source of X-rays and that we find it gives off three types of characteristic radiation which we may denominate the $\mathrm{K}, \mathrm{L}$, and $\mathrm{M}$ series of rays. The $\mathrm{K}$ type are harder than the L-rays and these in turn are harder than the soft M-rays. We choose the K-rays to work with; and we allow them to fall upon equal masses of a series of metals of increasing atomic weight.

On an earlier page it was pointed out that in order to excite the characteristic radiation of any element it was necessary to throw upon it X-rays of greater hardness than that of the element's own characteristic radiation. We begin by throwing the nickel $\mathrm{K}$ radiation on elements of an atomic weight less than that of nickel; and we find that the absorption of the element increases with the atomic weight, as shown by the rising curve marked $\mathrm{K}+\mathrm{L}+\mathrm{M}$ in Fig. 6. Over this range, the metals tested all emit their three types of characteristic radiation, $\mathrm{K}, \mathrm{L}$, and $\mathrm{M}$. Now, when we come to nickel itself (atomic weight $61 \cdot 3$ ) we find that there is a sudden drop in the absorption, as is indicated by the dotted line on the diagram. What has happened is that the nickel $\mathrm{K}$ radiation is now no longer able to excite the $\mathrm{K}$ radiation of the element on which it is falling,- since this element (nickel) would emit a $\mathrm{K}$ radiation as strong as that with which we are trying to excite it.

i Barkla and Sadler; Phil. Mag., 1909, 17, 739; Barkla and Collier, ibid., 1912, $23,987$. 
All that the incident nickel $\mathrm{K}$ radiation can now do is to excite the next element to emit the softer $\mathrm{L}$ and $\mathrm{M}$ radiations. The absorption of these increases with rise in the atomic weight of the absorbing element, as shown by the second up-grade of the curve (marked $\mathrm{L}+\mathrm{M}$ in the figure); until we reach an element with an atomic weight of approximately 160 . This element repeats the behaviour of nickel itself, for its $\mathrm{L}$ radiation is as strong as the nickel $\mathrm{K}$ radiation; and consequently all that the latter can do is to excite the soft M-rays. The results can be seen from the figure.

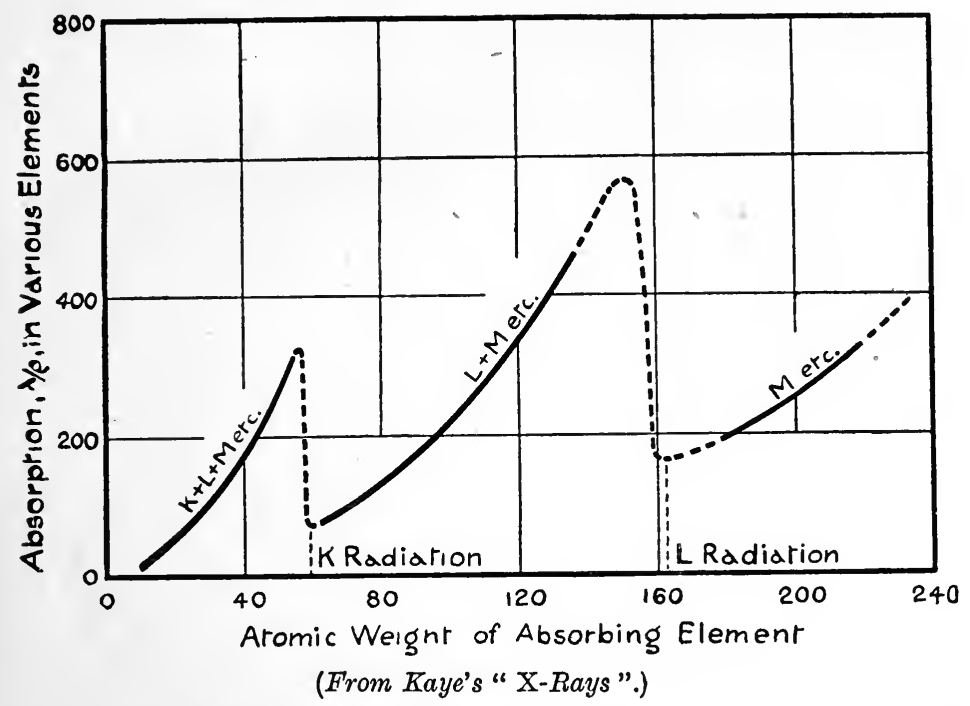

FIG. 6.-Graph showing relation between the absorption of nickel (K) radiation by various elements and the atomic weight of the absorbing element. The absorption passes through a minimum for a screen of nickel and also for one of atomic weight of about 164 (whose $L$ radiation is identical with the nickel (K) rays).

It is self-evident that if we used the $\mathrm{L}$ radiation of the element at atomic weight 160 as our exciting radiation, the results would be the same as those given above, since the hardnesses of the nickel $K$ and of the $L$ radiation from the 160 element are equal.

Benoist ${ }^{1}$ applied the absorption of X-rays to the solution

1 Benoist, Compt. rend., 1901, 182, 325, 545, 772; J. de Physique, 1901 , iii.), 10, 653 . 
of chemical problems in the following manner. If we cast a decigramme of a substance into the form of a right cylinder, the area of whose base is 1 sq. cm., and if we allow $\mathrm{X}$-rays of a given quality to fall normally upon the base and pass through the cylinder, the "specific opacity" of the material is measured by the fraction of the rays which is absorbed during their passage through the cylinder. Another constant is the "equivalent transparency" which is measured as follows. A cylinder of paraffin wax, $1 \mathrm{sq}$. cm. in cross-section and $75 \mathrm{~mm}$. high is chosen as the standard. The material to be examined is cast into a cylinder of equal cross-section. By experiment, the height of the second cylinder which will give an absorption equal to the wax is determined. The mass of the material in this size of cylinder is termed the "equivalent transparency" of the substance under examination. Both the specific opacity and equivalent transparency are properties independent of the state of aggregation and the temperature: and they are not affected by the state of combination in which the elements under examination exist.

Now Benoist showed that the specific opacity increases in a regular manner with the rise in atomic weight; so that if we plot specific opacity against atomic weight for a series of elements we get a smooth curve. Thus in order to determine whether an element has an atomic weight $\mathrm{A}$ or $\mathrm{nA}$, it is only necessary to establish its specific opacity and that of its possible neighbours. If the specific opacities lie on a smooth curve, the correct atomic weight has been found; if not, some multiple of this atomic weight must be selected and the elements with atomic weights near that must be examined until a smooth curve is obtained. Evidence of this kind has been found useful in establishing the position of indium in the Periodic Table.

\section{Corpuscular Rays.}

The corpuscular rays may be dismissed very briefly. It was shown by Curie and Sagnac ${ }^{1}$ that when an X-ray beam strikes a plate, part of the secondary radiation from the plate carries a negative charge. The intensity of the atomic

\footnotetext{
${ }^{1}$ Curie and Sagnac, Compt. rend., 1900.
} 
corpuscular radiation appears to be proportional to the fourth power of the atomic weight of the element from which the rays emanate $^{1}$; and the radiation becomes abundant when the material begins to emit its characteristic X-rays.

When the incident $\mathrm{X}$-ray is a characteristic X-ray-that is to say when it is a homogeneous radiation-the corpuscular ray excited by its impact on any given metal is also homogeneous in character. In each case, the corpuscular ray has a definite absorption coefficient which is proportional to the atomic weight of the metal whose characteristic rays were utilized as an exciter.

The fourth power relation mentioned above leads to interesting deductions. ${ }^{2}$ If we write $k$ for the absorption coefficient and $\mathrm{A}$ for the atomic weight of the metal emitting the exciting characteristic X-rays, we have the relation-

$$
k . A^{4}=\text { constant }
$$

Now by utilizing a formula deduced theoretically by Sir J. J. Thomson, the maximum velocity $\mathrm{V}_{a}$ with which a cathode ray can leave a material of thickness $d$ is given by

$$
\mathrm{V}_{0}^{4}-\dot{\mathrm{V}}^{4} a=a \cdot d
$$

where $\mathrm{V}_{0}$ is the initial velocity of the ray and $a$ is a constant. From this it follows that

$$
k \cdot v^{4}=\text { constant }
$$

where $v$ is the velocity of the particle at the moment of projection from the radiator. Combining the two expressions, it is clear that $v$ varies with the atomic weight.

Measurements by Beatty have shown that this velocity $v$ is equal to $10^{8} \mathrm{~A}$; and this turns out to be the critical velocity which a cathode ray must possess in order to be able to generate a characteristic radiation, as has been mentioned on an earlier page. Thus it appears that the corpuscular radiations have, approximately, the same velocity as the original incident cathode ray.

1 Whiddington, Proc. Roy. Soc., 1912, A, 86, 360; compare Moore, Proc. Roy. Soc., 1915, A, 91, 330, and Proc. Phys. Soc., 1915, 27, 432.

2 Ibid. 


\section{CHAPTER VIII}

\section{X-RAYS AND CRYSTAL STRUCTURE}

\section{The Phenomena of Diffraction.}

IN order to make clear the method by which the arrangement of atoms in crystals has been determined, it will be necessary to give a very brief account of the principles which underlie the action of diffraction gratings. In its simplest form, a diffraction grating may be regarded as a plane sheet of transparent material upon which are ruled a number of close-set, equidistant, and parallel lines. This construction results in the formation of a series of fine strips capable of reflecting or transmitting light, each strip being separated from its neighbours by a line which neither reflects nor transmits the lightrays which fall upon it.

In Fig. 7 let us assume that $\mathrm{AB}, \mathrm{CD}$, and $\mathrm{EF}$ represent sections of the opaque parts of the grating; whilst $\mathrm{BC}$ and $\mathrm{DE}$ are transmittent portions of the surface; and let us imagine that a series of parallel rays of homogeneous light fall from a slit at $\mathrm{S}$ upon the surface of the grating. When these light-rays reach the apertures $\mathrm{BC}$ and $\mathrm{DE}$ each wavefront becomes a centre of new vibrations in the ether which spread outward in all directions from the apertures as centres. Some of these rays will continue in a straight line perpendicular to the grating, as shown at BN. Others, however, will diverge in all directions; and for our present purpose we must consider the rays $\mathrm{BL}$ and $\mathrm{DM}$, one of which starts from $\mathrm{B}$ and the other from $\mathrm{D}$. These two rays are parallel and make an angle $\theta$ with the direct-passing ray BN. From D draw DK perpendicular to BL.

Now imagine that these rays are intercepted by a screen 
LM placed at right angles to the direction in which the rays are travelling. It will be evident from the figure that the ray from $B$ has to travel further than the ray from $D$ has to do before reaching the screen; since $\mathrm{BL}=\mathrm{BK}+\mathrm{KL}=\mathrm{BK}+\mathrm{DM}$. Thus a ray starting from $B$ has the extra distance $B K$ to pass over as compared with the ray from D. Suppose that BK is exactly equal to half a wave-length of the light used. Then clearly the ray from $\mathrm{B}$ will arrive at $\mathrm{L}$ in opposite phases of vibration from that in which the ray from $D$ reaches $M$ : if one arrives at L in its "crest" phase, the other will reach $M$ in the "trough" phase. And if these two beams be then concentrated by a lens, the two vibrations of the ether will neutralize one another and darkness will result. On the other hand, if the length $\mathrm{BK}$ be equal to a complete wavelength of the light, the two vibrations will arrive at $\mathrm{L}$ and $\mathrm{M}$ respectively in the same phase; and if they are condensed by a lens they will form together a bright image of the source.
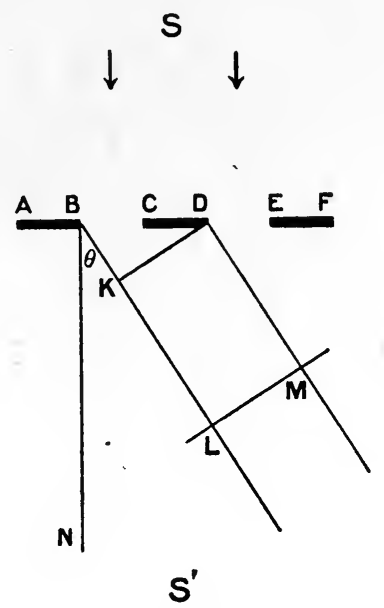

Fig. 7.

This reasoning can be applied more generally. If $\mathrm{BK}$ has a length equal to an odd number of half wave-lengths, extinction will result when the lens is applied: whilst if $\mathrm{BK}$ be equal to any number of complete wave-lengths the two vibrations will reinforce one another.

We must now consider the effect of the angle $\theta$ on the problem. From the figure, it is clear that the angle NBL is equal to the angle CDK; so that CDK also is $\theta$ and $B K$ is equal to $\mathrm{BD} \sin \theta . \mathrm{BD}$ is a fixed length, for it corresponds to the interval between two similar points in the grating system; but since we are at liberty to choose any of the rays which start in all directions from $B$ we can select any angle which we desire. If we pick out a particular angle $\theta$ which makes $\mathrm{BD} \sin \theta$ equal to an odd number of half wave-lengths, then we shall get extinction when the two rays from $B$ and $D$ are 
concentrated together; whilst if we choose $\theta$ so that $\mathrm{BD} \sin \theta$ equals a complete number of wave-lengths, we shall get an image formed by the lens. Further, if $\theta$ be chosen so that it lies between these extreme values, partial interference will result; and only a faint image will be formed by the lens. Finally, if the wave-length of the light which we use is longas in red light-BK also will be long when it is equal to a multiple of a complete wave-length; and hence $\sin \theta$ will be large and $\theta$ also will be large : so that the light of long wavelength will be diffracted far away from the direct beam $\mathrm{BN}$. A ray of short wave-length, on the other hand, will be much less diffracted, since in its case $\mathrm{BK}$ is small if it is equal to a multiple of the wave-length.

In practice, the light used is not homogeneous; so that, after diffraction, it is broken up into a series of spectra, each wave-length of which has its own angle for which the reinforcement of the various beams from the different apertures is greatest. This can be seen from a consideration of the formula $\mathrm{BK}=\mathrm{BD} \sin \theta$. Call the distance from $\mathrm{B}$ to $\mathrm{D} \delta$ and let $\lambda_{1}$ and $\lambda_{2}$ be the wave-lengths of light employed, then obviously the same value of $\theta$ will not satisfy both conditions; and we have the light diffracted at the angle $\theta_{1}$ in one case and at the angle $\theta_{2}$ in the second instance-

$$
\begin{aligned}
& \lambda_{1}=\delta \sin \theta_{1} \\
& \lambda_{2}=\delta \sin \theta_{2}
\end{aligned}
$$

In the foregoing reasoning we considered only one side of the apertures, but in practice diffraction takes place at each side of an aperture; so that two sets of spectra are formed, one on each side of the central beam.

Finally, we shall have one spectrum formed if the distance $\mathrm{BK}$ is equal to one wave-length; another spectrum is produced when BK is equal to two wave-lengths, and so on-

$$
n \lambda=\delta \sin \theta
$$

When $n$ is unity the corresponding spectrum is said to be of the first order; when $n$ equals 2 , the spectrum is termed a second order one, etc.

The results obtained with an actual grating are shown in Fig. 8.

The line 00 is the image formed by the direct, undiffracted 
beam $\mathrm{BN}$ and those parallel to it. Since all the wave-lengths of the source are represented in this beam, it is of the same nature as the light from the source itself. On either side of the central beam lie the two first order spectra, VR. Outside them lie the second order spectra, $V^{\prime} R^{\prime}$; and beyond them

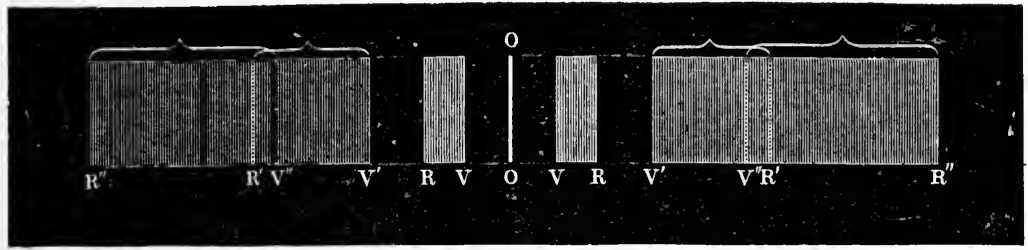

Fig. 8.-Grating Spectrum.

again, and partially overlapping them, will be seen the third order spectra, $V^{\prime \prime} R^{\prime \prime}$.

Hitherto we have dealt with diffraction caused by trans: mission through the grating; but it will be seen that the same reasoning holds good in the case of reflection from the surface of the grating. Turning again to Fig. 7 we need only imagine that light falls upon the grating from the source $S^{\prime}$ instead of from $S$; and that this light is absorbed by the portions $A B$ and CD whilst being reflected by the parts $\mathrm{BC}$ and $\mathrm{DE}$. The succeeding deductions are exactly analogous in this case to

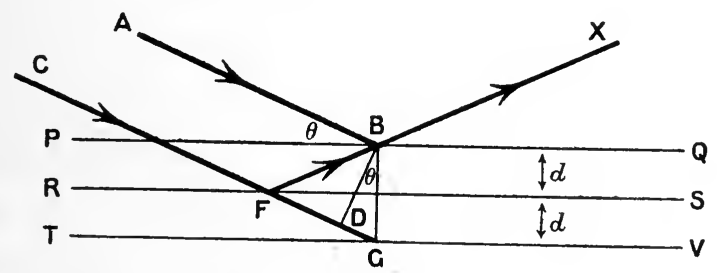

Fig. 9.

those which we have already drawn; so there is no necessity to go into them again in detail.

There is a third type of diffraction grating, different in nature from the two we have mentioned, which is specially important from the point of the present chapter. Let us assume (Fig. 9) that we have a series of parallel surfaces $P Q$ RS, TV, etc., which lie a certain distance $d$ apart from each 
other; and that they are composed of a material which is capable of reflecting some light and transmitting the rest of any beam which may fall on them. Assume, further, that parallel beams of homogeneous light fall upon this series of surfaces from the points $\mathrm{A}$ and $\mathrm{C}$. The beam from $\mathrm{A}$ strikes the upper surface at $\mathrm{B}$ : the beam from $\mathrm{C}$ penetrates the upper surface and is reflected from the second surface at F. Both beams will then travel along the line $\mathrm{BX}$. Produce $\mathrm{CF}$ to $\mathrm{G}$. $\mathrm{G}$ is obviously the image of $\mathrm{B}$ in the plane RS. Draw BD from $\mathrm{B}$ perpendicular to $\mathrm{CG}$. In the figure, $\mathrm{FB}=\mathrm{FG}$; and the angle $\mathrm{ABP}=$ the angle $\mathrm{DBG}$.

Now consider the distances travelled by the light-rays passing from $A$ and $C$ to $X$. In the one case, the path is $A B+B X$; in the other case it is $\mathrm{CF}+\mathrm{FB}+\mathrm{BX}$. Cancelling out the common part $\mathrm{BX}$ and substituting $\mathrm{CD}$ for $\mathrm{AB}$ and $\mathrm{FG}$ for $\mathrm{FB}$, we find that the difference between the two paths is DG, which may be expressed as $2 d \sin \theta$. Now if $\lambda$, the wave-length of the light we are using, be chosen equal to $2 d \sin \theta$, then the ray from $F$ will reinforce the ray from $B$; but if $\lambda$ has a value equal to a fraction of $2 d \sin \theta$, then interference will result and no clear image of the source will be formed at $\mathrm{X}$.

From this reasoning, it will be seen that if we know $\theta$ and $\lambda$ we can calculate $d$; or, knowing $d$ and $\theta$ we can calculate $\lambda$. The application of this will be seen in later sections. In the meantime, it will be sufficient to point out that here also various orders of spectra will be formed according to the value of the integer $n$ in the formula-

$$
n \lambda=2 d \sin \theta
$$

\section{Space Lattices.}

The distinction between an amorphous and a crystalline body lies in the fact that in the former the atoms and molecules are distributed at random, whilst in a crystal there is a certain regularity of arrangement according to a definite system. The investigation of such systems has been made possible by the work of Laue ${ }^{1}$ and especially by the researches of W. $\mathrm{H}$. and W. L. Bragg. ${ }^{2}$

1 Laue, Friedrich, and Knipping, Sitzber. K. Akad. Munchen, 1912, 303, 363.

2 W. H. and W. L. Bragg, $X$-Rays and Crystal Structure, 1915. A summary of this is to be found in a lecture by W. H. Bragg, Trans., 1916, 109, 252. 
Before entering into the subject, it will be necessary to obtain a clear conception of the meaning of the term "space lattice" since these space lattices form the basis upon which our ideas of crystal structure will be built up. A space lattice is an arrangement of points in space such that the environment about every point is the same as that about any other point. Suppose, for example, that we take a series of small cubes and assume that the points we are considering lie at the corners of the cubes. If we build the cubes together into a large block, it will be evident that the point at one corner of one cube has the other points of the system arranged about it in space in a certain pattern; and if we choose any other corner of any other cube it in turn will have other points arranged with regard to it in exactly the same manner as we found in the first case. In other words, each of these points has an environment of other points arranged in a particular way; and since the whole arrangement has a certain symmetry, it does not matter which point we fix upon to start with.

Now according to modern crystallographic theory, in any crystal the atoms of the same element are arranged so as to occupy the points of one or more such space lattices; and the whole crystal is made up by the interpenetration of a number of lattices. For example, in the case of a crystal of rubidium chloride, the positions of the rubidium atoms will represent points on one space lattice; the positions of the chlorine atoms will correspond to points on another space lattice: and the crystal will take the form of an arrangement built up by the interpenetration of these two space lattices.

We may look at the matter from another point of view. Imagine that we plot upon several planes an identical, symmetrical arrangement of points; and that we then place the planes parallel to one another in such a way that all the points form part of a symmetrical system. The points now make up a space lattice. If we arrange the points at the corners of imaginary squares upon the surface of the planes and then lay the planes parallel to each other so that a straight line can be drawn perpendicular to the planes through a set of points; and if we further arrange matters so that the distance between the planes is equal to the length of the side of the imaginary squares with which we started, then obviously the points in 
the three-dimensional figure will lie at the corners of cubes. This is what is called a cubic lattice. On the other hand, it is possible to conceive of an arrangement in which the lengths of the sides of the parallelopiped are all different and in which the lines joining similar points on different planes do not cut the planes at right angles. In this case we have the space lattice corresponding to a triclinic crystal.

\section{The Crystal as a Diffraction Grating.}

For the sake of simplicity, we shall confine the present section to the consideration of a cubic crystal; though the reasoning, with appropriate modification, will apply to any type of crystal.

As we have seen, a cubic crystal is assumed to be built up by the interpenetration of a number of space lattices, the points of any one lattice being occupied by the same type of atom. Suppose that a crystal contains sodium chloride. Then, in order to determine its structure, we begin by fixing the relative positions of, say, the sodium atoms. The atoms of chlorine will be arranged symmetrically with regard to the sodium atoms throughout the crystal structure; and we can determine their arrangement by a subsequent operation. In the case of the cubic system, the problem is simplified by the fact that only three types of lattice are possible: the simple cube with the atoms at its eight corners; the centred cube, which has an additional atom at its centre; and the facecentred cube with atoms at each corner of the cube and in the centre of each face, but with no atom in the cube's centre.

Imagine that we could pare off from the face of the crystal a slice of molecular thickness. In this slice-a "net" in crystallographic terms-we should find a regular grouping of atoms and spaces. Suppose that we were able to cut off from the slice a section, also of molecular thickness-a "row" as crystallographers name it-we should find its structure to be roughly representable by the following:-

Each of the points would represent an atom; and the spaces between the points would correspond to the spaces between the atoms in the crystal. This arrangement at once suggests 



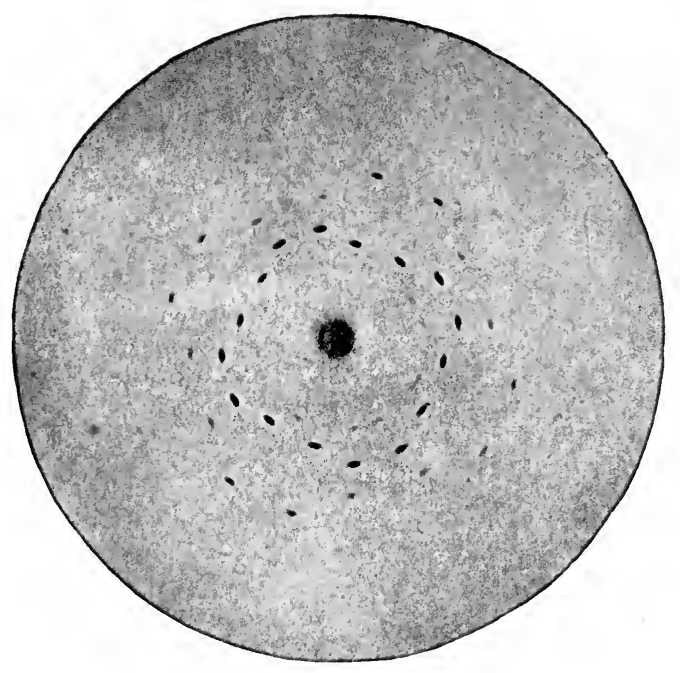

(From Kay'e's "X-Rays.")

FIG. 10. Pattern of Laue spots obtained by Friedrich and Knipping when $\mathrm{X}$-rays are diffracted by a zinc-blende crystal. The incident rays are parallel to a cubic axis of the crystal. 
a diffraction grating. If we could pass a ray of light through the crystal, the spaces between the atoms would play the part of the apertures in a diffraction grating; whilst if we reflected a ray of light from the crystal the solid atoms would act as the reflecting parts of a grating and the spaces would have no reflecting power. Thus it appears that in crystals we have a series of natural diffraction gratings lying parallel to each other and finer than any of our artificial structures.

In actual practice, when white light is thrown upon a crystal face we do not find the face acting as a diffraction grating. The grating is too fine as compared with the wavelengths of ordinary light to allow us to obtain the effects which we seek. It is therefore necessary to find some type of vibration with $\dot{a}$ wave-length very much shorter than that of even ultra-violet light. This vibration was discovered by Laue in the form of the X-rays.*

One important difference between the ordinary grating and the crystal grating must be mentioned. Owing to the fact that the crystal grating is three-dimensional, the reinforcement of one X-ray vibration by another is not always possible. A ruled grating, as we have seen, reflects a continuous spectrum when white light falls upon it; a crystal grating, on the other hand, reflects only certain specific wavelengths; so that it produces a discontinuous spectrum.

\section{Laue's Experiment.}

At the suggestion of Laue, Friedrich and Knipping investigated the effect of passing a fine pencil of X-rays through a crystal of zinc blende, the incident pencil being introduced parallel to an axis of symmetry of the crystal. Behind the crystal was placed a photographic plate; and when this was developed after the exposure, it was found to bear a symmetrical pattern of spots which is shown in Fig. 10 .

These spots are produced in the following manner. As the $\mathrm{X}$-ray pulse passes over the atoms of the zinc blende, each atom becomes a centre of new vibrations, just as each point

* The discovery was really due to the fact that Laue was searching for some means of diffracting X-rays and bethought himself of crystals as likely to fulfil his purpose. 
on the wave-front of a light-pulse is a centre of fresh ethereal movement. An X-ray spectrum is thus thrown out by the three-dimensional grating of the crystal; but owing to the extra dimension, as has been pointed out, this is not a continuous spectrum, for it consists of a few lines only. Each spot on a quadrant of the figure represents a particular wavelength; and the central dark disc is formed by the undiffracted portion of the X-rays.

Laue put forward an explanation of the phenomenon which was based on the assumption that the X-ray pencil was made up of a limited number of different wave-lengths: but his views have since been shown to be erroneous: and for the true theory of the matter we are indebted to W. L. Bragg.

\section{The X-ray Spectrometer.}

Just as an ordinary spectroscope serves for the examination of light spectra the Bragg X-ray spectrometer ${ }^{1}$ enables us to investigate the $\mathrm{X}$-ray spectra of elements and compounds. The following brief description will make its construction clear (see Fig. 11) :-

The $\mathrm{X}$-rays are generated by a bulb in which the anticathode is placed perpendicular to the cathode stream; and the particular X-rays used are those which leave the anticathode at the grazing angle-i.e. those which are travelling almost parallel to the surface of the anti-cathode. These rays pass through a fine slit in the lead casing with which the bulb is screened; and a second slit is sometimes introduced in their path in order to obstruct any scattered radiations which may have filtered through the first protection. In the path of the ray, after it has passed through the slits, is placed the crystal to be examined. It is mounted on a revolving table by means of a lump of wax. The pencil of X-rays emerging from the crystal is passed into an ionization chamber which revolves on the same axis as that which carried the crystal. The ionization chamber is closed and contains a gas easily ionizable by $\mathrm{X}$-rays, such as sulphur dioxide; and the $\mathrm{X}$-ray enters the chamber through an aluminium window before which a third slit is placed. The ionization chamber is insulated and maintained

1 See Bragg, X-Rays and Crystal Structure, chapter iii. 


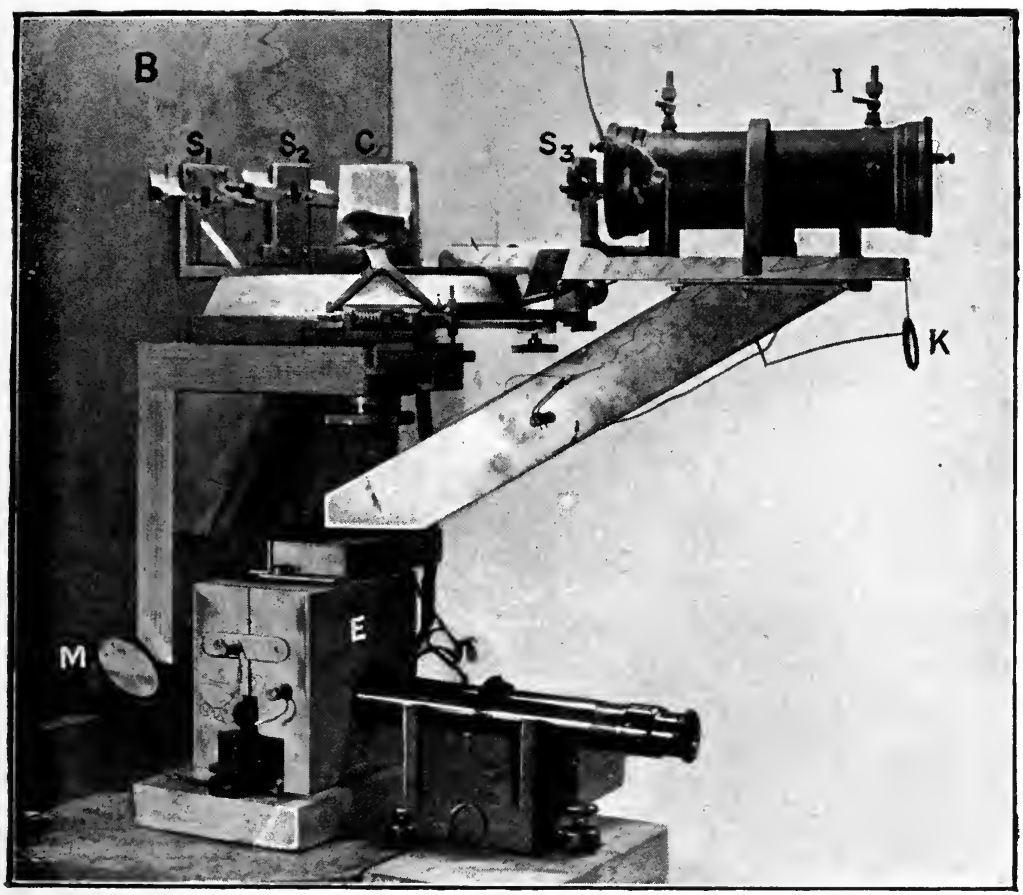

$$
\text { (From Kaye's "X-Rays.") }
$$

FIG. 11.-Photograph of Bragg's X-ray spectrometer. B is a lead box containing an $\mathrm{X}$-ray bulb. $\mathrm{S}_{1}$ and $\mathrm{S}_{2}$ are adjustable slits which direct a beam of X-rays on to the face of the crystal C. The reflected beam passes through the slit $S_{3}$ into the ionisation chamber $\mathrm{I}$, where it is recorded by the tilted electrocsope in the metal box $\mathrm{E}$. $\mathrm{K}$ is an earthing key; $\mathrm{M}$ a mirror for illuminating the electroscope. $\mathrm{C}$ and I can each be rotated about the axis of the spectrometer. 

at a high potential by means of storage cells. It contains an electrode mounted just out of the path of X-rays entering the chamber; and this electrode is connected with a Wilson tilted leaf electroscope.

The operation of the apparatus is almost self-evident. Suppose that the crystal be kept fixed and the X-ray stream is turned on. The ionization chamber is then moved slowly round the axis; and whenever a strong $\mathrm{X}$-ray penetrates the chamber, the gas in the latter becomes ionized and the electroscope is affected. The angle of the ionization chamber is read off; and from it the wave-length of that particular ray can be determined in terms of the crystal grating. The ionization chamber is then rotated through a further angle until the electroscope is again affected, showing that a new $\mathrm{X}$-ray is passing through the chamber. It will be evident that the base of the ionization chamber is moving along the circumference of a circle over which the X-ray spectrum is being distributed by the crystal grating.

The position in which the ionization chamber rests when ionization is observed gives a means of calculating the angle at which the X-ray has been refracted from the crystal; and the degree of ionization-determined by the rate of discharge of the electroscope-is a measure of the intensity of the particular line which is entering the spectrometer.

\section{The Analysis of Crystal Structure.}

Again, for the sake of simplicity, we shall confine our attention to cubic crystals. Such crystals are assumed to be built up from a series of elementary cubes; and the pattern on which they are constructed must fulfil the requirements of cubic symmetry. Now if we examine the conditions which fulfil these demands, we shall find that there are three possible arrangements of the characteristic points within the elementary cube-

Type 1. The points lie at each corner of the cube.

Type 2. There are points at each corner of the cube and also a point at the centre of the cube.

Type 3. There are points at the corners and also in the centre of each cube face. 
In order to determine the nature of a crystal we must define two factors-

A. The space lattice of each element in the crystal.

B. The nature of the interpenetration of the various space lattices.

The results which were obtained in the cases of sodium chloride and the chloride, bromide, and iodide of potassium will serve as an example of the method employed. From the similarity in chemical character of the substances, it might appear probable that all the salts would give the same Laue pattern; but when the examination of them was made it was

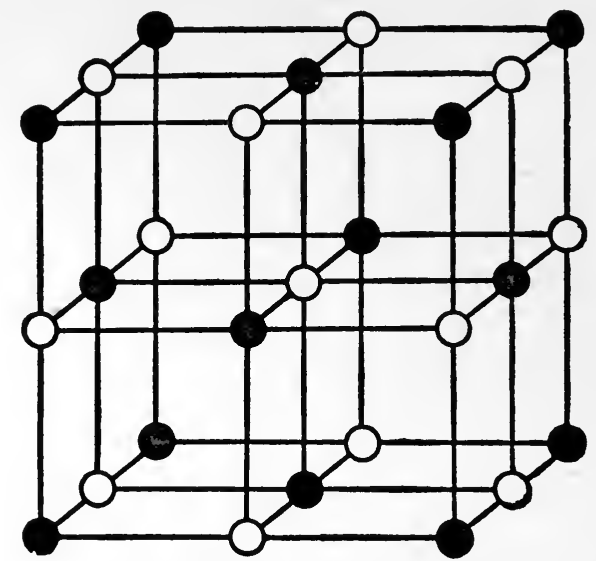

(From Kaye's " $X$-Rays".)

Fig. 12.-Representation of two types of diffracting centres in a cubic crystal.

found that they differed markedly from one another. Potassium chloride gave rise to a simple pattern of spots such as would be formed by a cubic lattice of the first type. Potassium bromide and potassium iodide produced a spot-pattern characteristic of the face-centred lattice described in Type 3. Sodium chloride yielded a pattern more complex than either of the others and apparently intermediate* between them. How can we explain this?

Let us examine the system illustrated in Fig. 12.

* Strictly speaking, sodium chloride is more like potassium bromide; but the small difference between the diffracting powers of sodium and chlorine atoms produces an apparent resemblance to the potassium chloride struct ure. 
In this diagram, the characteristic points are represented by light and dark circles. Let us first ignore the distinction between the two and look at the figure as a whole. It will be evident that it represents a space lattice of the first type, since all the points are situated at the corners of cubes. Now omit from consideration the white circles, and it will be found that the black circles are placed at the corners of the large cube and also in the centres of the faces of that cube. In other words, this arrangement represents a space lattice of the third type. Further, the white circles taken alone form parts of two exactly similar groupings.

We must now endeavour to interpret this in terms of the results given by the salts. Let us make a single assumption : that a heavy atom forms a better centre of diffraction than a light atom does. This assumption leads us to draw a distinction between the constituents of the various salts which have been described; for we should expect to find a marked difference betrveen the diffraction effects of potassium (atomic weight 39 ) and iodine (atomic weight 127); but only a slight difference between those of potassium and chlorine (atomic weight $35 \cdot 5)$.

Take first the case of potassium chloride. Here the two constituent atoms are of approximately equal weight; and their diffractive powers, in accordance with our assumption, will be of almost equal value. The Laue figure produced will depend upon the joint action of the two. If we return to Fig. 12 we shall see that when the black and white circles are of equal value, the whole system becomes a Type 1 lattice; so that it is not a far-fetched assumption if we regard the potassium chloride crystal as being built up in this manner, the two different kinds of circle representing the potassium and chlorine atoms.

Now take the case of potassium bromide and potassium iodide. Here we have the light potassium atom and the comparatively heavy bromine and iodine atoms. The Laue figure produced by these salts will be influenced mainly by the heavy atoms. We find that the pattern actually produced is characteristic of a face-centred lattice; and from this we must deduce that the halogen atoms occupy the places indicated by the black circles in Fig. 12. 
Finally, in the case of sodium chloride, the difference in atomic weight between metal and halogen is not so great as in potassium iodide but is greater than in potassium chloride; and hence, according to our assumption, there will be an impress of both sodium and chlorine effects upon the plate in the Laue pattern. But the result of this will be to give us a pattern which will be akin to the face-centred variety, if we regard the chlorine atoms only, or will be related to the cubic lattice if we assume that chlorine and sodium have approximately equal effects. In other words, we shall have a mixture

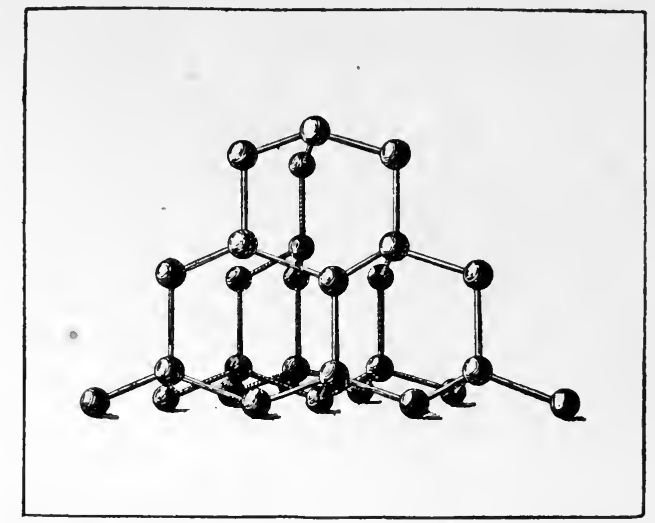

(From Bragg's "X-Rays" (Geo Bell \& Sons, Ltd.).) FIg 13.

of patterns which corresponds exactly to the experimental result. Rubidium bromide and crsium iodide should give the same pattern as potassium chloride; and an examination of these two substances will be of much interest.

There seems to be little doubt that the assumption of the space lattice shown in Fig. 12 as a representation of the alkali halides, is fully justified.

The results obtained with other crystals may be described very briefly. Curiously enough, the face-centred lattice appears to be a very common one; for the crystals of copper, silver, zinc blende, iron pyrites, fluorspar, and the diamond all appear to be constructed upon this system or by the interpenetration of more than one such system.

The zinc blende crystal is composed of a face-centred 
lattice of zinc atoms combined with a face-centred lattice of sulphur atoms, the latter grouping proceeding from coincidence with the zinc one along a diagonal of the face-centred cube to approximately a quarter of the length of the diagonal.

Diamond is an exceptionally interesting crystal from the point of view of chemical theory. In the model illustrated in Figs. 13 and 14, it will be seen that every atom stands at the centre of gravity of a tetrahedron formed by its four nearest neighbours; and, further, that the atoms are arranged in rings

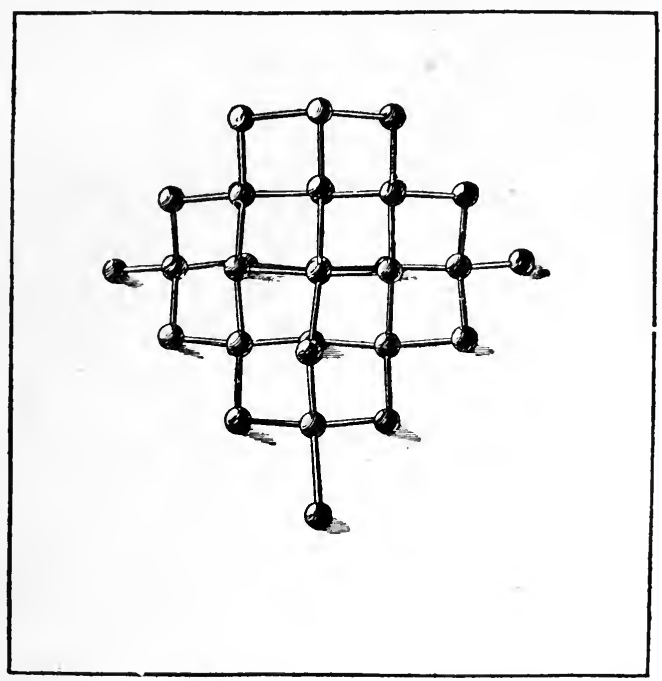

(From Bragg's " X-Rays" (Geo. Bell \& Sons, Ltd.).)

FIG. 14.

of six throughout the structure. The suggestiveness of this grouping in connection with the benzene theory ${ }^{1}$ and the tetrahedral arrangement of optically active carbon compounds requires no elaboration here. From the point of view of the space lattices, it is sufficient to note that the diamond arrangement may be considered as derived from the zinc blende construction by the substitution of two similar atoms for the dissimilar zinc and sulphur atoms of zinc blende.

In calcium fluoride, the fluorine atoms lie at the centres of small cubes into which the larger face-centred cubes of calcium

1 See Collie, Trans., 1916, 109, 561. 
can be divided. The grouping in iron pyrites cannot be made clear without a figure. ${ }^{1}$

The copper crystal is perhaps the simplest of all. It is based on a face-centred lattice; and the copper atoms occupy the same positions relative to one another as would be taken up by a series of shot piled upon one another.

We must now turn to another unknown in the problem: the several distances which separate the atomic planes lying parallel to the crystal faces. The reasoning in this case is rather more complicated. ${ }^{2}$ We begin by deciding to use throughout the experiments one particular wave-length of $\mathrm{X}$-ray, say the $\mathrm{B}$ peak from a platinum anti-cathode. In the formula-

$$
\lambda=2 d \sin \theta
$$

the only factor which we can determine directly is $\theta$, which we can measure by means of the X-ray spectrometer; the other two factors, $d$ and $\lambda$ are left unevaluated for the present.

Now if we apply the X-ray successively to the three primary planes of a crystal, we can obtain three different values of the expression $\frac{d}{\lambda}$. Call these three values $\frac{d_{1}}{\lambda}, \frac{d_{2}}{\lambda}$, and $\frac{d_{3}}{\lambda}$. Since $\lambda$ is constant throughout, these values give us the relative lengths of the sides of the elementary parallelopiped from which the crystal is built up.

Further, the volume of this elementary parallelopiped is given by $\frac{d_{1} \times d_{2} \times d_{3}}{\lambda^{3}}$ which is simply $\frac{\mathrm{V}}{\lambda^{3}}$. Let us take another step. If the density of the crystal be $\rho$, then the mass associated with each elementary parallelopiped will be $\mathrm{V} \rho$; and if $\mathrm{M}$ be the molecular weight of the substance, the number of molecules in each parallelopiped is $\frac{V_{\rho}}{M}$. Write this in the form $\lambda^{3}\left(\frac{\mathrm{V}}{\lambda^{3}} \cdot \frac{\rho}{\mathrm{M}}\right)$; then the quantity within the brackets is proportional to the number of molecules in each parallelopiped.

1 See Trans., 1916, 109, 265.

2 For the following simple method of presentation I am indebted to Kaye's $X$-Rays, p. $221 \mathrm{ff}$. 
When the value of $\left(\frac{\mathrm{V}}{\lambda^{3}} \cdot \frac{\rho}{\mathrm{M}}\right)$ is calculated from the experimental data for such different substances as sodium chloride, zinc sulphide, calcium fluoride, calcium carbonate, and iron pyrites, it is found that in each case it approximates to unity. In the case of sodium chloride, for example, it is 1.22. This points to there being only a single molecule associated with each diffracting centre; and if we combine this with the reasoning already given with regard to the nature of the space lattices of the alkali salts, it seems reasonable to deduce further that there is only one heavy atom associated with each characteristic point in the lattice. Support for this view is found in the case of potassium chloride, for-when its constant is calculated it is found to be 0.605 ; which implies that there is only half a molecule-i.e. either a potassium or a chlorine atom-at each characteristic point in the lattice. It will be recalled that this conclusion has already been drawn from data of a totally different type.

If the foregoing reasoning be correct, then obviously $\frac{\mathrm{V} \rho}{\mathrm{M}}=1 ;$ and we can write-

$$
\lambda^{3}\left(\frac{\mathrm{V}}{\lambda^{3}} \cdot \frac{\rho}{\mathrm{M}}\right)=1
$$

In the case of sodium chloride we have-

Molecular weight, $\mathrm{M}=58.5 \times 1.64 \times 10^{-24} \mathrm{grs}^{*}{ }^{*}$

$$
\begin{aligned}
\text { Density } \frac{\rho}{V} & =2.15 \text { gr. } / \text { c.c. } \\
\frac{V}{\lambda^{3}} & =33.3 \text { (determined experimentally). } \\
\lambda^{3}(33.3 \times 2.15) & =58.5 \times 1.64 \times 10^{-24} \\
\text { and } \lambda & =1.10 \times 10^{-24} .
\end{aligned}
$$

This gives the wave-length of the homogeneous $B$ platinum $\mathrm{X}$-ray. From this we can now calculate the value of $d$ in the equation $\lambda=2 d \sin \theta$.

In the case of graphite, a measurement made on the cleavage basal plane showed that in this instance $d$ is $3.42 \times 10^{-8} \mathrm{~cm}$. In the calcite crystal a measurement of the plane (100) yielded $3.04 \times 10^{-8} \mathrm{~cm}$. for the value of $d$; whilst for the planes (110) $d$ was found to be $1.917 \times 10^{-8} \mathrm{~cm}^{1}{ }^{1}$

* The mass of a hydrogen atom is taken as $1.64 \times 10^{-24}$ grammes.

1 Bragg, X-Rays and Crystal Structure, pp. 115-16. 


\section{CHAPTER IX}

\section{X-RAY SPECTRA AND ATOMIC NUMBERS}

\section{The Method of Measuring X-ray Spectra.}

THE work of W. H. and W. L. Bragg, described in the last chapter, provided a means of determining the wave-length of any particular X-ray, and thus opened the way to an examination of the X-ray spectrum emitted when a given substance is excited by the ordinary methods. The investigations of Moseley ${ }^{1}$ in this field resulted in the discovery of a new atomic relation which appears to be of fundamental importance; and their extension by other workers ${ }^{2}$ has thrown light upon a number of points of great interest.

The method employed by Moseley was as follows. The stream of electrons from the cathode of an X-ray tube was concentrated upon a small area of an anti-cathode composed of the element whose high-frequency spectrum it was desired to examine. The characteristic rays thus set up were allowed to pass through a slit in a platinum plate, after which they emerged from the X-ray tube through an aluminium window. In their path, inclined at a certain angle, was placed the cleavage face of a crystal of potassium ferrocyanide which analysed the X-ray beam. After being diffracted by the crystal, the rays were allowed to fall upon a photographic plate; and in this way photographs of the X-ray spectrum were

1 Moseley, Phil. Mag., 1913, 26, 1024 ; 1914, 27, 703. Lieutenant Moseley was killed during the Dardanelles campaign, 1915.

2 de Broglie, Compt. rend., 1913, 156, 1153 ; 1916, 165, 87, 352; de Broglie and Lindemann, ibid., 1913, 156, 1461 ; Herweg, Verh. deut. phys. Ges., 1914, 16, 73; Siegbahn and Friman, Physikal Zeitsch., 1916, 17, 17, 48, 61; Siegbahn and Stenström, ibid., 318; Siegbahn, Ber. deut. phys. Ges., 1916, 18, 39, 150, 278; Siegbahn and Friman, Phil. Mag., 1916, 31, 403 ; Friman, ibid., 1916, 32, 497 : cf. Rutherford and Andrade, ibid., 1914, 27, 854. 



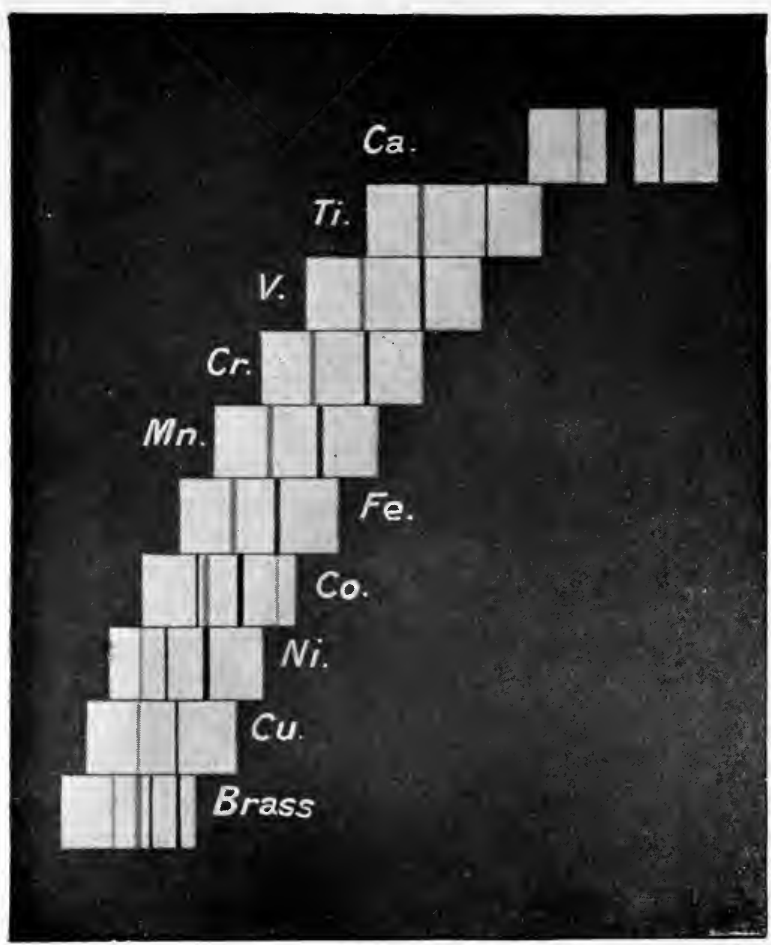

- Increasing Wave Length.

(From Kaye's "X-Rays.")

FIG 15.-Moseley's photographs of the X-ray or high-frequency spectra of a number of metallic anticathodes. The spectra, which are the third order, are placed approximately in register in the figure. For each metal, the more intense line, with the longer wave-length, is the $\mathrm{K}$ characteristic radiation. The brass shows the $\mathrm{Zn}$ and $\mathrm{Cu}$ lines; the cobalt contained both nickel and iron as impurities. 
obtained. Owing to the weakness of certain of the radiations, the spectrometer was constructed so as to be capable of evacuation; and it thus became possible to photograph even those $\mathrm{X}$-rays which would have been intercepted by a layer of air.

From the photographs thus obtained, the wave-lengths (or the frequencies) of the $\mathrm{X}$-rays emitted by the anti-cathode could be conveniently measured.

\section{The Nature of the X-ray Spectra.}

It will be remembered that the characteristic rays emitted by various elements fall into series which are termed the $K$, $\mathrm{L}$ and $\mathrm{M}$ series. Moseley showed that the $\mathrm{K}$ spectrum of all the elements contains two strong lines; and an examination of Fig. 15 will show that a certain regularity can be traced as we pass from element to element. The strongest line in the spectrum is termed the $a$-line and the weaker one is named the $\beta$-line. Inspection of the figure will suffice to prove that in the case of the cobalt spectrum the original specimen has been contaminated with nickel and iron (since the spectrum contains weak lines characteristic of these elements); whilst the nickel spectrum contains traces of the copper spectrum also.

Later work has shown that the spectra are not quite so simple as they were at first supposed to be; for more lines have been detected which were missed by Moseley. The spectra obtained when X-rays of the $\mathrm{L}$ series are used instead of the $K$ series are still less simple. But even the most complex X-ray spectra are simplicity itself compared with ordinary light spectra; and the mathematical analysis of them is therefore much more likely to yield us definite information with regard to the systems of vibration which produce them.

In the case of gaseous elements such as chlorine, it might be supposed that experimental difficulties would hinder the determination of the X-ray spectrum; but it has been found that such elements can be dealt with by utilizing a compound instead of the element itself. Potassium chloride, for example, shows the line spectrum of potassium and in addition it exhibits certain non-potassium lines which are evidently due 
to the chlorine atom in the molecule. In this way it is possible to establish the high-frequency spectra of gaseous and liquid elements; though of course the method breaks down in the case of the inert gases which form no compounds.

We must now turn to the problem presented by the frequencies of the $\boldsymbol{a}$-lines in the spectra of various elements. If we calculate for each element the factor $Q$ from the equation-

$$
\mathrm{Q}=\sqrt{\frac{\nu}{\left(\frac{1}{1^{2}}-\frac{1}{2^{2}}\right)} \nu_{0}}=\sqrt{\frac{\nu}{\frac{3}{4} \nu_{0}}}
$$

in which $v$ is the frequency of the $a$-line in the X-ray spectrum of the element in question, whilst $\nu_{0}$ is the fundamental frequency of ordinary line spectra which was obtained by Rydberg,* there is obviously a certain regularity in the results-

\begin{tabular}{|c|c|c|c|c|}
\hline . & & Q. & Atomic Weight. & Atomic Number. \\
\hline $\begin{array}{l}\text { Titanium } \\
\text { Vanadium } \\
\text { Chromium } \\
\text { Manganese } \\
\text { Iron . }\end{array}$ & $\dot{\text { : }}$ & $\begin{array}{l}20 \cdot 99 \\
21 \cdot 96 \\
22 \cdot 98 \\
23 \cdot 99 \\
24 \cdot 99\end{array}$ & $\begin{array}{l}48 \cdot 1 \\
51 \cdot 06 \\
52 \cdot 0 \\
54 \cdot 93 \\
55 \cdot 84\end{array}$ & $\begin{array}{l}22 \\
23 \\
24 \\
25 \\
26\end{array}$ \\
\hline
\end{tabular}

Examination of the figures shows that there is no direct relation between the factor $Q$ and the atomic weight for any given element. $\dagger$ At the same time, there is a certain regularity in the values for $Q$; for each of them differs approximately by unity from its neighbours above and below. Further, the elements, when arranged in order of their $Q$ values, lie in the order which is suggested by their chemical properties.

If we now turn to the Periodic Table and, beginning with

* It may be noted in passing that Moseley used Rydberg's earlier result, $\left(\nu_{0}=109,720\right)$ instead of the more recent value $\nu_{0}=109,675$, but the difference between the two is slight.

$\dagger$ The relationship : Atomic weight $=2 Q+6 \cdot 12$, which might be deduced from the figures given above, breaks down when the series is extended. $E . g$. for silver, $Q=46 \cdot 6$, whence $2 Q+6 \cdot 12$ would be $99 \cdot 32$ instead of $107 \cdot 88$. Harkins and Wilson (J. Amer. Chem. Soc., 1915, 37, 1396) have proposed the formula: Atomic weight $=2\left(\mathrm{~N}+n^{\prime}\right)+\frac{1}{2}+\frac{1}{2}(-1)^{\mathrm{N}-1}$ in which $\mathrm{N}$ is the atomic number and $n^{\prime}$ is zero for the lighter elements. 
hydrogen as No. 1, count along the Table until we arrive at titanium, we shall find that it is the twenty-second element in the series; vanadium is the twenty-third; chromium is the twenty-fourth, and so on. Here also we have a series of figures each differing from its neighbours by unity; and the arrangement of the elements in each of the two series is the same. The numbers obtained by counting along the Table from hydrogen are termed Atomic Numbers. ${ }^{1}$ Inspection will show that for any element in the above series the factor $Q$ and the atomic number $\mathrm{N}$ are approximately related thus-

$$
\mathrm{N}=\mathrm{Q}+1
$$

Looking at the matter from a more general point of view, it is clear that $Q, v$, and $\nu_{0}$ in the equation given above are related to one another in the following manner:-

$$
v=\frac{3}{4} \nu_{0} \times Q^{2}
$$

In the case of the $\mathrm{L}$ series spectra, the relationship takes the form -

$$
\mathrm{Q}=\sqrt{\frac{v}{\left(\frac{1}{2^{2}}-\frac{1}{3^{2}}\right) \nu_{0}}}
$$

so that in that case the relation between $\mathrm{Q}$ and the other factors become-

$$
\nu=\frac{5}{36} \cdot \nu_{0} \times \mathrm{Q}^{2}
$$

Evidently the most general way of writing the relationship is-

$$
\nu=\mathrm{AQ}^{2}
$$

and since $Q$ is a factor which increases concurrently with the atomic number $\mathrm{N}$, we are entitled to substitute $\mathrm{N}-b$ for $\mathrm{Q}$, if $b$ is a constant. The relation between the frequency of the $a$-line of an element and the atomic number of the element thus becomes simply-

$$
\nu=\mathrm{A}(\mathrm{N}-b)^{2}
$$

and in the case of the $K$ line it can be seen from the figures given above that $b=1$; so that the expression is reduced to-

$$
\nu=A(N-1)^{2}
$$

' van den Broek, Physikal. Zeitsch., 1913, 14, 33 ; Nature, 1913, 93, 373, 476; Soddy, ibid., 399, 452 ; Rutherford, ibid., 423 ; Bohr, Phil. Mag., 1913, 26, 1. 
Moseley ${ }^{1}$ suggested that the integer $\mathrm{N}$ was identifiable with the number of free positive units of electricity contained in the atomic nucleus, which brings his work into line with previous view of Soddy's that the chemical nature of an element depends, not on its atomic weight, but upon its electrical character ${ }^{2}$ : the mass of the atom being regarded as of only secondary importance.

\section{The Gaps in the Periodic Table.}

We must now turn to another region of the subject. It is clear that if we determine the atomic numbers of any two elements from their X-ray spectra we are in a position to state the number of other elements which are interposed between them. For example, in the table given on p. 116 titanium is the twenty-second element and iron is the twenty-sixth; therefore there must be three elements between titanium and iron, corresponding to the numbers 23,24 , and 25 . These elements are of course, vanadium, chromium, and manganese.

The atomic number of uranium has been found to be 92 , so that there can be only ninety elements between it and hydrogen. Of these ninety, we already know eighty-four, so that there are still six to be accounted for. A glance at the Periodic Table will show that there is a blank space between molybdenum and ruthenium, where an element with an atomic number 43 may be expected. Another space lies between tungsten and osmium, corresponding to the atomic number 75 . A third blank in the table is located between polonium and niton; and a fourth between niton and radium. This leaves us with a couple of unknown elements still to be allocated. Moseley assumed that thulium was a mixture of two elements, one of which is thulium proper and the other is the celtium of Urbain; and he placed the remaining missing element, No. 61, between neodymium and samarium. ${ }^{3}$

The importance of X-ray spectra in this field can hardly be over-estimated. The rare earth group of elements has long

1 Moseley, Nature, January 5th, 1914.

2 Soddy, Jahrb. Radioaktiv. Elektronik, 1913, 10, 193 ; Nature, December 4th and 18th, 1913; Chemistry of the Radio-Elements, II., 6 (1914).

'See Soddy; Chemical Society's Annual Reports, 1914, XI., 279, for information supplementing Moseley's papers in the Phil. Mag. 
been one of the puzzles of chemistry; and the definite proof of the exact number of these substances still remaining to be discovered has removed the possibility that much time and labour might be wasted in seeking for fresh members of the group after the roll-call was complete. The rare earth group stands apart from the remainder of the Periodic Table and contains within itself no indication of the number of elements which it includes; so that without Moseley's key we might have seen many fruitless attempts to discover "missing" members long after the whole flock was gathered together in the fold.

\section{The Anomalies of the Periodic Table.}

It is well known that the Periodic Table is not free from blemishes in its arrangement. Argon and potassium do not follow one another in the order of their atomic weight; nor is the chemical sequence of iron, cobalt, and nickel the same as the order of increasing atomic weight. Iodine and tellurium are also inverted in the usual form of the Table.

Moseley's work has shown that the atomic numbers run parallel to the chemical sequence of the elements and not to their order of atomic weights. For example, the X-ray spectra show that the value of $\mathrm{N}$ for potassium is 19 , which brings it into Group I as usual, instead of placing it in Group $\mathrm{O}$ as the atomic weight sequence does. Also, the atomic order of the iron group is : iron (26); cobalt (27); and nickel (28): which agrees with their chemical behaviour and not with their arrangement by increasing atomic weight. Tellurium and iodine also fall into their chemical order. This supports what has already been said with regard to the importance of the atomic numbers from the chemical standpoint.

Another point of interest arises in connection with certain "elements" which, on stellar spectroscopic evidence, have been supposed to exist. Coronium, nebulium, and asterium have all been taken for granted on the strength of certain lines which were observed in the spectra of the heavenly bodies; and places were assigned to elements of this class in a special series between hydrogen and lithium. If Moseley's conclusions are correct, there can be no such series; and evidence of the 
existence of the celestial elements will in future be regarded with considerable suspicion.*

A crucial test of the correctness of Moseley's atomic numbers was furnished by the case of the isotopes. $t$ These are elements having different atomic weights, but possessing properties so similar on the chemical side that they cannot be separated from one another by any reactions. If the atomic number is a function of that portion of the atomic structure which manifests itself in chemical properties, then clearly the spectra of two isotopes ought to be identical. The matter has been put to the test by Rutherford and Andrade. ${ }^{1}$ Lead and radium- $B$ are chemically inseparable from one another; and when their X-ray spectra were compared, it was found that they were identical. Thus each group of chemically inseparable elements has a single atomic number covering the whole group and the individual isotopes are not entitled to separate en umeration.

\section{The Spectrum of Hydrogen.}

An interesting point arises when we consider the X-ray spectrum of hydrogen. This has not been observed; and it cannot be calculated exactly from Moseley's formula because when $\mathrm{N}$ is put equal to unity in that equation, the quantity within the bracket becomes zero and no physical meaning can be attached to the result. The information can, however, be obtained by another method which leads to an approximation.

Moseley suggested that the square root of the frequency of the radiation was probably proportional to the nuclear charge on the atom; and for nuclear charge we may substitute atomic number. In the present case it will be convenient to substitute for the frequency its reciprocal, the wave-length of the vibration.

* It must, however, be borne in mind that if the "astronomical elements" are isotopes (see Chapter XVII.) of the elements in the first series of the Table, the above conclusion could not be sustained, since two isotopes have the same atomic number; but if the spectra of two isotopes be identical (see p. 200), the existence of the new celestial elements isotopic with known elements can hardly be supported by spectroscopic evidence.

+ See Chapter XVIII.

1 Rutherford and Andrade, I'hil. Mag., 1914, 27, 854 ; see also ibid., 1916, $32,49$. 
We thus get the following equation, which is approximately true :-

$$
\sqrt{\frac{\nu_{1}}{\nu_{2}}}=\sqrt{\frac{\lambda_{2}}{\lambda_{1}}}=\frac{N_{1}}{N_{2}}
$$

Now in this equation we replace $\mathrm{N}_{2}$ by 74, the atomic number of tungsten ; $\lambda_{2}$ we replace by $0.185 \times 10^{-8} \mathrm{~cm}$., which is the wave-length of the $K$ radiation of tungsten; and we substitute 1 , the atomic number of hydrogen, for $N_{1}$. This gives us the value of $\lambda_{1}$ which proves to be $101.3 \mu \mu$. In other words, the wave-length of the $K$ radiation emitted by the hydrogen atom should approximate to $101 \cdot 3 \mu \mu$.

Now the shortest wave-length discovered by Lyman in the remote ultra-violet spectrum of hydrogen is $91.2 \mu \mu$; and there is good reason for supposing that this is the shortest vibration of which a hydrogen atom is capable. Bearing in mind the approximate character of the calculation we have made, the agreement in value between the experimentally determined ultra-violet wave-length and the wave-length calculated for the $K$ radiation of hydrogen seems to suggest that the ultra-violet spectrum of hydrogen is simply the X-ray spectrum of the gas. For the $\mathrm{L}$ radiation a similar calculation can be made, which brings out a value closely approximating to the ordinary visible spectrum of hydrogen.

From this it follows that in all probability the ordinary radiations of hydrogen are neither more nor less than the element's X-ray spectrum. Very little thought will show how important this conclusion may be from the point of view of our knowledge of the relations between atomic constitution and spectral series.

\section{Molecular Numbers.}

Allen ${ }^{1}$ has suggested that just as we have atomic weights and molecular weights, so we ought to consider atomic numbers and "molecular numbers," the latter being obtained by adding together the atomic numbers of the atoms in any molecule just as the molecular weight is got by taking the sum of the atomic weights of the atoms composing the molecule. The molecular

* The expression $\mu \mu$ represents a millionth of a millimetre.

1 Allen, Trans., 1918, 113, 389. 
number would represent the number of positive charges on the various atomic nuclei in the molecule. Allen points out that if we consider the four compounds: $\mathrm{CH}_{4}, \mathrm{NH}_{3}, \mathrm{H}_{2} \mathrm{O}$, and $\mathrm{HF}$ which are formed by the combination of hydrogen with a typical element belonging to successive groups in the Periodic Table, all four compounds have the same molecular number, 10. Similarly all the compounds: $\mathrm{SiH}_{4}, \mathrm{PH}_{3}, \mathrm{H}_{2} \mathrm{~S}$, and $\mathrm{HCl}$ have the molecular number 18 in common. The two series differ by eight units; and this difference, or one of eighteen units, makes its appearance frequently in comparisons among molecular numbers.

A point of some interest is found when the molecular number of ammonium is calculated. It is found to be 11 , which is identical with the atomic number of sodium. In view of the resemblances between the sodium and ammonium ions, the coincidence of the values is striking.

It is too early yet to express any opinion on the value of the molecular numbers in chemical problems; but when they have been more exhaustively studied it seems possible that valuable results may be deduced from their relations.

\section{Conclusion.}

Enough has now been said to indicate the importance of Moseley's work ; and it seems probable that we cannot at present appreciate the developments which may arise from it in the near future. It is true that X-ray spectra have, on further investigation, proved to be more complicated than was at first supposed; but even at their greatest complexity they are much simpler than the corresponding emission spectra of the elements ; and their mathematical treatment, complex as it may prove to be, will certainly be a much easier problem than the attack upon the luminous spectra has been.

The relation between the X-ray spectra and the model atom of the physicists is of considerable interest; but a discussion of the question would lead us far beyond the limits laid down for the present chapter.

Allen ${ }^{1}$ has shown that there is a simple relation connecting the atomic number $\mathrm{N}$, the characteristic atomic frequency $\nu$,

${ }^{1}$ Allen, Proc. Roy. Soc., 1917, A, 94, 100; Phil. Mag., 1917, 34, 488. 
and a fundamental frequency $v_{\mathrm{A}}=21.3 \times 10^{1} \mathrm{sec}^{-1}$. He finds that this relationship can be expressed by the equation-

$$
\mathrm{N} v=n \nu_{\mathrm{A}}
$$

The value $n$, which he terms the frequency number, is always an integral. For the applications of this to problems of atomic heats and photo-electric phenomena, the original papers should be consulted.

On the purely chemical side, the support lent by Moseley's work to the Periodic Arrangement of the elements cannot but give rise to much speculation. To what particular property does the atomic number correspond?

In the first place, it has no traceable relation to the maximum valency exhibited by an element; for in the rare earth group we find a set of elements all of which are obviously trivalent, and yet their atomic numbers run in sequence just as if they were characteristic elements of the first two series in the Periodic Arrangement.

Secondly, the fact that lead and radium- $B$ have the same $\mathrm{X}$-ray spectrum, though their atomic weights are not identical, appears to prove that there is no direct relationship between the atomic weight and the atomic number.

Thirdly, since the radio-active properties of lead and radium- $B$ are different, it is clear that the atomic number is not affected by those factors in an atom which give rise to radio-active phenomena.

This leaves us with only one characteristic of the elements undiscarded: the chemical properties. But here we are still left in doubt. The chemical properties of ferrous iron and ferric iron are quite different; so are the properties of copper in the cuprous and cupric forms. Can we assume that the atomic number of ferrous iron is the same as that of ferric iron? If so, then the atomic number cannot be regarded as a guide to chemical sequence in the strictest form. It will be of considerable interest to see the results which are obtained with the X-ray spectra of elements in the "ous" and "ic" varieties; for it seems probable than an investigation in this direction will enable us still further to limit our search for the property which corresponds most closely to the atomic number. 


\section{CHAPTER X \\ THE ELEMENTS OF THE RARE EARTHS}

\section{Introductory.}

In the atomic order between barium and tantalum there is a group of elements whose atomic weights lie between 130 and 180. These substances, together with scandium and yttrium, make up the greater part of what are termed the rare earths.* These rare earths are found for the most part in the Scandinavian peninsula, but deposits have also been brought to light in the Ural Mountains, and in certain parts of America and Australia. It appears that rare earths are usually found among eruptive rather than among sedimentary deposits, and that they are most likely to occur in ancient igneous rocks, particularly in granite.

The rare earths are chiefly made up of a series of basic oxides, but it is usual to divide them into three groups: the cerium group, the terbium group, and the ytterbium group. This division is arbitrary, for the rare earth elements resemble each other very closely indeed, and we have really a single series of substances with properties changing slightly. as we pass from member to member, rather than three groups whose properties differ sharply from each other. $\dagger$ Still, for the sake of convenience, the following arrangement is usually employed :-

* The term " rare earths" is a misnomer in modern times, as it has been found that these substances are really very widely distributed in nature and extensive deposits of them are known at the present day. Detailed accounts of the rare earth group are to be found in Eriend's Textbook of Inorganic Chemistry, vol. iv., by H. V. F. Little, and in Spencer, The Rare Earth.

$\dagger$ The solubility of double salts is the chief criterion applied in arranging the elements into these three groups. 


\begin{tabular}{|c|c|c|c|c|}
\hline \multicolumn{2}{|c|}{ Cerium Group. } & Terbium Group. & \multicolumn{2}{|c|}{ Ytterbium Group. } \\
\hline $\begin{array}{l}\text { Atomic } \\
\text { Number. } \\
\text { 57. Lanthanum } \\
\text { 58. Cerium } \\
\text { 59. Praseodymium } \\
\text { 60. Neodymium } \\
\text { 61. Sa } \\
\text { 62. Samárium }\end{array}$ & $\begin{array}{l}\text { Atomic } \\
\text { Weight. } \\
.139 \cdot 0 \\
.140 \cdot 25 \\
.140 \cdot 9 \\
.144 \cdot 3 \\
. ? \\
.150 \cdot 4\end{array}$ & $\begin{array}{lr}\text { Atomic } & \text { Atomic } \\
\text { Number. } & \text { Weight. } \\
\text { 63. Europium } & .152 \cdot 0 \\
\text { 64. Gadolinium } & 157 \cdot 3 \\
\text { 65. Terbium } & .159 \cdot 2\end{array}$ & $\begin{array}{l}\text { Atomic } \\
\text { Number. } \\
\text { 66. Dysprosium } \\
\text { 67. Holmium } \\
\text { 68. Erbium } \\
\text { 69. Thulium } \\
\text { 70. Ytterbium } \\
\text { 71. Lutecium } \\
\text { 72. Celtium. } \\
\text { 39. Yttrium }\end{array}$ & $\begin{array}{c}\text { Atomic } \\
\text { Weight. } \\
\text { m } 162 \cdot 5 \\
.163 \cdot 5 \\
.167 \cdot 7 \\
.168 \cdot 5 \\
.173 \cdot 5 \\
.175 \\
. \quad ? \\
. \quad 88 \cdot 7\end{array}$ \\
\hline
\end{tabular}

Assuming that Urbain's celtium has the atomic number 72, this leaves only one rare earth still unknown, and it should correspond to the blank position, No. 61 .

The group of the rare earths has considerable importance, whether we look upon the question from the point of view of theory or from the practical standpoint. With the theoretical problem involved we shall deal in Section 5 of Chapter XIX, so we need not enter here into the question of the position which must be assigned to these elements in the Periodic System.

On the practical side, the rare earths were without any importance until a quarter of a century ago; but in 1884, Auer von Welsbach took out a patent in which the presentday incandescent gas mantle was foreshadowed, and at once the question of the rare earths became a commercial one. At that time the known deposits were limited, both in extent and number, and it consequently was doubtful whether the raw material for the manufacture of mantles could be produced at a price sufficiently low to allow of commercial success. Fortunately, large deposits of monazite sand were soon afterwards discovered in Carolina and in Brazil. These beds rendered it possible to obtain the rare earths comparatively cheaply, and the incandescent gas-lighting industry has grown up on them.

At this point the theoretical side has shared in the profit, for the mantle industry has produced vast quantities of byproducts which have been placed at the disposal of investigators, who would otherwise have been forced to work up the crude material for themselves at the cost of much time and 
labour. At the present day, these by-products form one of the most fruitful sources in the investigation of the elements of the rare earth series.

\section{The Physical Properties of the Rare Earth Elements.}

Muthmann and Weiss ${ }^{1}$ have carried out a comparison between some of the members of this group of substances, and have obtained the following results :-

As regards colour, lanthanum is a white metal similar to tin in appearance; cerium is rather more like iron; neodymium shows a faint tinge of yellow; while praseodymium is more pronouncedly yellow in $t_{i}:$ it.

When the hardness of these elements is compared with that of lead, tin, and zinc, they lie in the following order: lead, tin, cerium, lanthanum, zinc, neodymium, praseodymium. Samarium is harder than any of the foregoing.

The melting-points are shown in the following table. Aluminium melts at about $660^{\circ} \mathrm{C}$., and silver at about $960^{\circ} \mathrm{C}$., which gives a rough standard of comparison :-

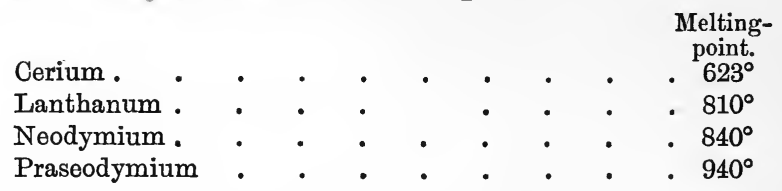

The specific gravities of the four metals are-

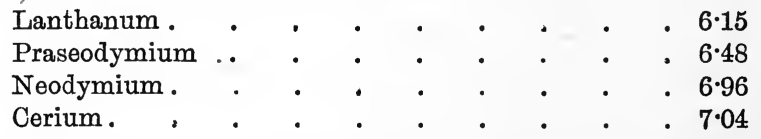

Cerium, therefore, has about the same density as tin $(7 \cdot 3)$.

\section{The General Chemical Character of the Rare Earth} Elements.

The metals of the rare earths are comparatively reactive, and enter into direct combination with various elements. They burn in air, ${ }^{*}$ giving rise to oxides of the type $\mathrm{Me}_{2} \mathrm{O}_{3}$; but in addition to this series, some of the metals can be converted

1 Muthmann and Weiss, Annalen, 1904, 331, 1.

* Cerium burns with a brilliance exceeding even that of magnesium. 
into oxides having the composition $\mathrm{MeO}_{2}$. For example, cerium oxide of the formula $\mathrm{Ce}_{2} \mathrm{O}_{3}$ is unstable, the stable oxide being $\mathrm{CeO}_{2}$. The corresponding oxide of praseodymium has also been isolated, but it is much less stable than the cerium one. Terbium and neodymium also yield higher oxides having the compositions $\mathrm{Tb}_{4} \mathrm{O}_{7}$ and $\mathrm{NdO}_{2}$. It is a curious fact that there are two forms of the oxides $\mathrm{Me}_{2} \mathrm{O}_{3}$, one form being prepared by heating the nitrate of the metal, the other form being obtained by igniting the hydroxide. The two varieties are different in both physical and chemical properties. It appears likely that this case is parallel to that of the two forms of calcium oxide (unslaked lime and overburned lime) and to the two varieties of magnesium oxide. The origin of the difference in properties probably lies in the existence of two different polymorphous forms of the oxides.

The oxides of the rare earth metals are bases of medium strength ; the order of basicity appears to be the following, the strongest bases being placed first :-

$$
\begin{gathered}
\mathrm{La}_{2} \mathrm{O}_{3}, \mathrm{Pr}_{2} \mathrm{O}_{3}, \mathrm{Nd}_{2} \mathrm{O}_{3}, \mathrm{Ce}_{2} \mathrm{O}_{3}, \mathrm{Y}_{2} \mathrm{O}_{3}, \mathrm{Sm}_{2} \mathrm{O}_{3}, \mathrm{Gd}_{2} \mathrm{O}_{3}, \mathrm{~Tb}_{2} \mathrm{O}_{3}, \\
\mathrm{Ho}_{2} \mathrm{O}_{3}, \mathrm{Er}_{2} \mathrm{O}_{3}, \mathrm{Tm}_{2} \mathrm{O}_{3}, \mathrm{Yb}_{2} \mathrm{O}_{3}, \mathrm{Sc}_{2} \mathrm{O}_{3}, \mathrm{CeO}_{2}
\end{gathered}
$$

The behaviour of ignited lanthanum oxide resembles that of quicklime; it hisses when placed in contact with water, and absorbs carbon dioxide from the air. The oxides of the ytterbium series are much less active in this respect. From the fact that their salts with strong acids are not measurably hydrolysed even in dilute solution, it follows that the oxides of the cerium group are the strongest bases derived from trivalent elements.

The rare earth metals combine directly with hydrogen and nitrogen as well as with oxygen. The hydrides have the general formula $\mathrm{MeH}_{2}$ or $\mathrm{MeH}_{3}$, and are obtained either by passing hydrogen over the metals at $200^{\circ} \mathrm{C}$. to $300^{\circ} \mathrm{C}$, , or by the action of magnesium upon the metallic oxides in a stream of hydrogen. The nitrides are formed by the action of nitrogen upon the oxide in presence of magnesium, or from the carbides by the action of ammonia. They have the composition $\mathrm{MeN}$.

The carbides of the rare earth series have the general formula $\mathrm{MeC}_{2}$. They are produced by the electrolytic reduction of the oxides in presence of carbon; and when treated with 
water they yield mixtures of hydrogen with acetylene and other hydrocarbons.

It is unnecessary for us to enter into details with regard to the salts of these metals. They present no special characteristics. The rare earth elements form salts with nearly all the ordinary organic and inorganic acids, and these salts possess to a great extent the ordinary properties of salts of strong bases.

\section{Methods of Purifying the Rare Earths.}

In the present section no attempt will be made to enter into any great detail in the description of the various methods employed to divide the rare earths from the minerals among which they occur, and to separate the elements of this group from one another. For these details the reader is referred to larger treatises. ${ }^{1}$ All that we can do in this place is to indicate briefly the main lines of the separations, choosing as far as possible the more characteristic of these.

There are four chief stages in the isolation of the rare earths from the deposits in which they occur naturally-

A. The decomposition of the mineral, the isolation and conversion into oxalates of the rare earth class.

B. The conversion of the rare earth oxalates into soluble salts.

C. The separation of the rare earth group into three subgroups by means of double salt formation with potassium sulphate.

D. The isolation of the various elements from the cerium, terbium, and ytterbium sub-groups.

Section D we cannot enter into in detail, as each element requires special methods for its treatment. We shall content ourselves with indicating in outline the principles underlying the methods actually employed.

A. In nature, the rare earths occur among silicates, which are usually easily broken up by evaporating the ore to dryness with concentrated hydrochloric or sulphuric acid. The powdered residue is then added to water, and any insoluble portion is filtered off. The solution now contains the rare earths in the

1 See especially Little's account of the rare earths in vol. iv. of Friend's Textboole of Inorganic Chemistry (1917). 
form of chlorides or sulphates, and may also contain salts of copper, bismuth, molybdenum, and iron. Thorium also is often present. The solution is treated with sulphuretted hydrogen to precipitate the metals of the second group. This leaves us with the rare earth elements, iron, and thorium in the solution. After oxidation of the ferrous iron to ferric with chlorine, we add a little hydrochloric acid, and then precipitate the rare earth elements and thorium in the form of oxalates.

Other reagents are sometimes utilized to break up the mineral. Fusion with potassium hydrogen sulphate or with sodium hydroxide, heating with hydrofluoric acid or with sulphur dichloride vapour are processes which have advant ges in special cases.

B. Since the oxalates are insoluble substances, we must convert them into some other salts which we can get into solution. This is usually done by one of three methods. The oxalates may be heated to form oxides, from which any required salt can be produced; or we may simply dissolve the oxalates in hot nitric acid, and thus obtain the easily soluble nitrates; or, finally, we may boil the oxalates for a considerable time with caustic potash solution, which gives us the hydroxides, and from these we can produce any salt we may need. At this point we have to get rid of the thorium which still remains in the solution. This is usually done by adding hydrogen peroxide to the nitrate solution; after warming, a precipitate of thorium peroxide separates out and is filtered off.

C. We have now to separate the rare earth salts in three main groups by means of double salt formation with potassium sulphate. It has been found that in this way we get the following classification:-

1. Elements giving practically insoluble double salts : Scandium, cerium, lanthanum, praseodymium, neodymium, and samarium.

2. Elements giving soluble double salts : Europium, gadolinium, and terbium.

3. Elements giving easily soluble double salts: Dysprosium, holmium, erbium, thulium, yttrium, ytterbium.

Instead of potassium sulphate, sodium sulphate is sometimes used.

D. When we come to the actual isolation of one element of the rare earth series from its companions, there are two chief 
methods which we may employ. The first of these depends upon the basicities of the different metals; the second upon the differences in the solubility of their salts. The former method is capable of subdivision; for we may depend either upon a fractional precipitation of the salts by means of bases of different strengths, or we may rely upon a differential decomposition of the nitrates by means of heat. We must now give an outline of these methods-

1a. The principle underlying the method of fractional precipitation is almost self-evident. If we take two substances $\mathrm{X}$ and $\mathrm{Y}$ in solution, and add to them a third substance $\mathrm{Z}$ which is capable of precipitating both $X$ and $Y$, then if we add excess of $\mathrm{Z}$, both $\mathrm{X}$ and $\mathrm{Y}$ will be completely deposited from the solution. If, on the other hand, we add a quantity of $Z$ which is insufficient to precipitate the total quantity of $\mathrm{X}$ and $\mathrm{Y}$ present, it is clear that the amounts of $\mathrm{X}$ and $\mathrm{Y}$ precipitated will depend to a great extent upon their chemical behaviour with respect to $Z$. Suppose that $Z$ is a base which is very much stronger than $A$, but only very little stronger than $B$. If we have one molecule of each body present, it is clear that $\mathrm{Z}$ will displace $\mathrm{A}$ from its salts rather than B. Consequently we shall have A precipitated and $B$ left in solution. In the case of the rare earths, this method is employed, using such reagents as ammonia, magnesia, caustic potash, caustic soda, or organic bases. In the first precipitation the substance is separated into two parts: precipitate and mother liquor. The precipitate is then redissolved, and the process is repeated. The crystals resulting from the second fractional precipitation are once more dissolved and fractionally precipitated, and the process is continued until a pure substance is obtained.

1b. The second method, depending upon the difference in basicity of the various members of the rare earth group, is carried out in the following manner. In the first place, the mixed oxalates are converted into nitrates in the usual way, and to this mixture is added some alkali nitrate in order to lower the melting-point of the whole mass. Thereafter the mixture is fused. It is then found that the uitrates decompose, and usually the most negative oxide separates out first. This is removed from the mass by solution, and the process repeated again and again as in the last case. 
2. The method depending upon the solubilities of the salts of the rare earths is simply a process of fractional crystallization. The mixture of salts is dissolved in water, and the solution is then evaporated until about half the solid separates out. This is filtered off and dissolved in water, and the same process is repeated. At the same time the mother liquor is again evaporated till half its solute separates out, and the process is repeated a sufficient number of times. Where there are more than two elements present, it is usual to mix the fractions in the following manner: After the first crystallization, we have crystals (A) and mother liquor (B). In the second set of crystallizations, each of these gives rise to a set of crystals and a mother liquor. The crystals derived from the second crystallization of the mother liquor (B) are then mixed. with the mother liquor derived from the recrystallization of (A), and in this way a third set of fractions is produced which grow more and more rich in the salt whose solubilities lie between the two extremes. The scheme below will make the point clear-

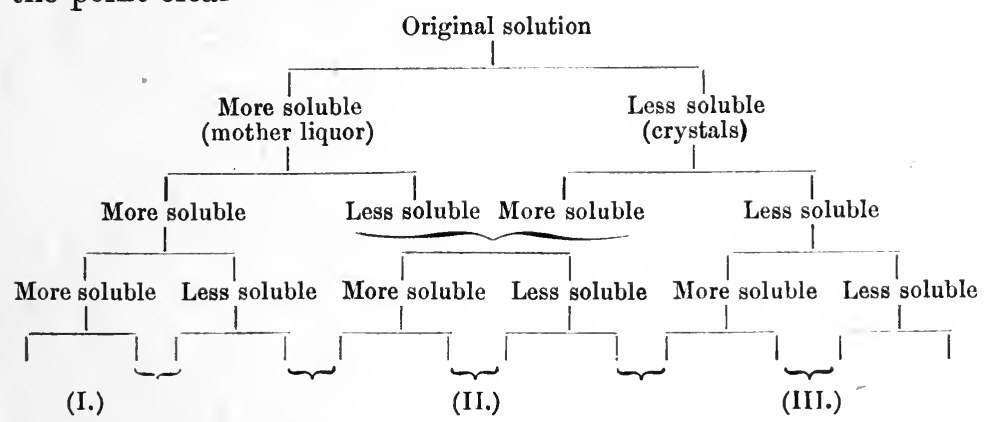

The brackets indicate that two fractions have been mixed together before recrystallization.

A glance at the scheme will show that we are accumulating the more soluble salts at (I.), the salts of intermediate solubility at (II.), and the least soluble salts at (III.).

The salts which have been found most useful in this method of separation are the chromate, sulphate, nitrate, oxalate, and formate. The metallic derivatives of acetylacetone have also been utilized. James ${ }^{1}$ has discovered

1 James, Chem. News, 1907, 95, 181; 1908, 97, 61, 205 ; J. Amer. Chem. Soc., 1907, 29, 495. See also ibid., 1908, 30, 979, for his general scheme of 
two methods by means of which rapid separations of the yttrium earths can be carried out. In the first of these, the rare earth oxalates are dissolved by warming them with a saturated solution of ammonium carbonate in dilute ammonia; and the fractionation is carried out by simply boiling the solution until a precipitate is thrown down. If five fractions of approximately equal weight be obtained in this manner, the first contains yttrium, the second is mainly holmium and dysprosium, the third is a mixture of various elements, while the last two contain erbium. James' second method depends upon the fractionation of the bromates of the rare earths; he obtains these from the corresponding oxalates by treating the latter with sulphuric acid, and then with barium bromate.

Now, let us suppose that we have completed a series of fractional precipitations or crystallizations. The question at once arises: Have we carried the process to its end and secured the pure product we set out to obtain? In order to answer this question, we must know whether or not we are altering the composition of our substance in the course of the fractionation. Thus quite early in the series of fractionations it becomes advisable to find out what change each successive operation produces in the substance under treatment.

There are three methods by means of which we may settle the question. If we are gradually purifying a crude substance, it is obvious that if, after each operation, we determine the equivalent of the metal (or the average equivalent of the mixture in the first case) we shall get a gradual approximation to the equivalent of the pure metal, as the fractions contain less and less impurity the further the process is continued. For example, if we start with a mixture of cerium and lanthanum, the equivalent of the mixture (if the two metals be present in equal proportions) will lie half-way between those of cerium and lanthanum. As our fractions become richer and richer in cerium, the equivalent of the fraction will draw closer and closer to the cerium equivalent; and when the fraction contains nothing but cerium salt, we shall, of course, find the equivalent of cerium. This method is very rough at the best; and when applied to the case of the rare separation. For a full account of the subject, see Little's chapters in Friend's Textbook of Inorganic Chemistry, vol. iv., especially p. $322 \mathrm{ff}$. 
earths it is unsatisfactory, owing to comparatively small differences between the equivalents of the various metals in the group.

The second method, which is much more refined, consists in applying the spectroscope to the problem. Here we have a considerable choice of procedure, for either absorption or emission spectra may be used; and in the latter class there are three different types: spark spectra, are spectra, and phosphorescence spectra. The spectrum of each set of fractions is examined and purity is counted as attained when repeated fractionation fails to alter the spectrum.

The third method depends upon the fact that the rare earth elements differ considerably from one another in their magnetic susceptibilities; so that the determination of this property suffices as a guide to the purity of the various fractions. ${ }^{1}$

\section{Industrial Applications of the Rare Earth Elements.}

The main employment of rare earth elements is to be found in the incandescent gas-mantle industry, which utilizes cerium and "didymium"* salts to a certain extent, as will be seen in the next chapter. The quantities required by the trade, however, are comparatively small; and owing to the fact that large quantities of cerium are isolated as by-products during the extraction of thorium in the mantle industry, it has been worth while to look for other uses for cerium.

Cerium, in the form of alloys, has been utilized in various ways. An alloy of cerium with lanthanum, praseodymium and neodymium is known technically as mischmetall; and it is utilized as a reducing agent. The well-known cerium tinders, which are used in gas-lighters or cigarette-lighters, are alloys of cerium with 30 per cent. of iron, nickel, cobalt, or other metals ; and the use of these is extending rapidly.

Cerium is also used for impregnating carbon electrodes for

1 Urbain, Compt. rend., 1908, 146, 406, 922 ; 1909, 149, 37 ; 1910, 150, 913 ; 1911, 152, 141 ; Urbain and Jantsch, ibid., 1908, 147, 1286 ; Blumenfeld and Urbain, ibid., 1914, 159, 323. mium.

* The so-called " didymium" is a mixture of neodymium and praseody- 
arc lamps; and it is claimed that this treatment yields a more brilliant and steady arc than ordinary carbon.

Cerium glass has the power of cutting off about 30 per cent. of the heat rays and all the ultra-violet portion of the spectrum; so that for certain purposes it is very useful.

Other uses for cerium have been suggested in photography, dyeing, medicine, and catalytic processes; but the field appears to be limited. 


\section{CHAPTER XI}

\section{INCANDESCENT GAS MANTLES}

\section{Introductory.}

THE fact that the introduction of solids into a non-luminous flame may cause the emission of light is common knowledge to any one who has ever heated a platinum wire in the jet of a Bunsen burner; but between this experiment and the production of an efficient system of illumination on a commercial scale lie a long series of steps; and it is only within the present generation that the process of perfecting the incandescent gas mantle has been completed.

In 1829, Berzelius observed that oxides like thoria or zirconia emitted a brilliant light when placed in non-luminous flames; but the first practical application of the idea appears to have been made when lime-light came into use. Lime heated in an oxy-hydrogen flame gives out a brilliant light; but for ordinary illuminating purposes it was useless, owing to the fact that ordinary gas-flames are too low in temperature to produce a satisfactory radiation from lime.

The incandescent mantle industry, 1 in its modern form, dates from 1884, when Auer (later Auer von Welsbach) took out a patent protecting the use for illuminating purposes of fibrous materials impregnated with oxides of certain rare elements. By 1891 the incandescent mantle had become a practical possibility; but at this point the fortunes of the new industry hung in the balance. Though the supply of rare earths was then amply sufficient for the purposes of scientific investigation, it seemed doubtful whether enough

1 Fuller details of the industry are to be found in Martin's Industrial Inorganic Chemistry, and similar works. 
material could be obtained to cope with the requirements of a commercial process. At the time when the new mode of illumination was placed on the market, the minerals upon which it depended cost over a sovereign an ounce, and, what was even more grave, they could be obtained only in small amounts, twenty or thirty pounds being a very fair find at one time in the Norwegian deposits.

The demand for incandescent mantles soon made it clear that fresh sources of supply must be found, if the process was to keep the position which it had gained; and a search for other deposits was begun. It was recalled that thorium had been detected in certain abandoned gold claims in Carolina; and soon the monazite sand of that district was found to be a new mine of rare earths. Indeed, so great were the quantities discovered there and in Brazil that the epithet "rare" ceased to bear its primitive meaning. Ceylon has also yielded a material called thorianite, which is rich in thorium oxide. Within ten years, owing to the discovery of these new sources, the price of the rare earths dropped to 2 or 3 per cent. of its original amount; and the permanency of the incandescent gasmantle industry was assured, so far as this factor was concerned.

\section{The Treatment of Monazite Sand.}

Natural monazite sands, of course, vary in composition according to their place of origin; but for the purpose of illustration, the Brazilian type will serve our purpose. The percentage of the mineral monazite in the sands may vary between 2 and 60 per cent.; and the remainder of the sand is valueless from the commercial point of view. Even monazite itself contains a large percentage of worthless material; for its thorium content is only 5 per cent. of the whole mass. Now in order to make the material economically valuable for export, it is necessary to concentrate the thorium up to at least 4 per cent. strength : in other words, a product must be prepared which contains about 90 per cent. of pure monazite.

The raw sand contains, in addition to monazite, quantities of quartz mixed with lesser amounts of other minerals; and the exact nature of the purification process depends largely 
upon the kind of impurities which are present. Three main methods of segregation are employed: (1) the wet process; (2) dry blowing; and (3) electro-magnetic separation.

In the wet process, the sand is mixed with water and the liquid is run over a vibrating table, by which means the sand is sifted out into groups according to the specific gravity of its particles. Since quartz has a density of 2.65 whilst monazite's density is 4.8 to $5 \cdot 5$, it will be seen that the separation of the two is not difficult. The process of dry blowing depends upon the same principle, but an air-blast is substituted for the stream of water. In the electro-magnetic method, advantage is taken of the fact that the ingredients of monazite sand are differently affected by a magnet. The sand is fed on to the top of a revolving belt and thrown from this against another travelling belt behind which a strong electromagnet is placed. The magnetic attraction retards the velocity of certain of the sand particles while leaving others to travel forward unaffected; and by means of a series of slots leading to bins, the various constituents of the sand are roughly separated from each other.

The next stage in the concentration takes the form of "breaking" which consists in feeding the monazite, obtained as above, into cast-iron pans containing twice its weight of hot sulphuric acid (specific gravity 1.84). Heating completes the process and converts the monazite into a pasty mass. This mass is run into cold water and any insoluble materials such as silica are allowed to settle to the bottom. The solution, which contains the rare earth phosphates dissolved in dilute sulphuric acid, is siphoned off from the insoluble matter. In this way a material is obtained which contains thoria and the rare earths in the proportions of $1: 12$.

Further concentration of the thoria is effected by taking advantage of the fact that thorium is more basic than its companions and separates before them when the solution is largely diluted or is neutralized with ammonia or magnesite. The thorium precipitated in this way is filtered off, dissolved in the minimum quantity of acid, and reprecipitated by a repetition of the process.

This leaves us with a concentrated preparation of thorium phosphate and the phosphates of some rare earth elements, from 
which phosphoric acid is removed by methods which are trade secrets.

Thereafter, the purification of the individual constituents of the mixture is carried out by methods which were outlined in the preceding chapter.

\section{Fabrics and their Treatment.}

Three fabrics have been utilized in the manufacture of mantles: cotton, ramie fibre, and artificial silk. Of these, cotton is the least satisfactory and artificial silk the best, though its cost hinders its general employment. Ramie fibre is prepared from China grass; whilst artificial silk may be obtained either from viscose or from cuprammonium cellulose.

The first step in the preparation of the mantle is the testing of the yarn from which the mantle is to be made; for the knitting is done by machinery, and the tension to which the yarn is subjected appears to have some effect upon the final product. The knitting machine is designed to produce a continuous cylindrical fabric which can be cut up into lengths as required.

Artificial silk contains no mineral matter; but both cotton and ramie fibre are contaminated with inorganic and fatty impurities which must be removed. Cotton mantles are therefore treated with dilute caustic soda solution and thereafter are washed in dilute acid. Ramie fibre fabric is soaked for some hours in dilute acid; freed from excess acid by centrifuges; washed in pure water and dipped in dilute ammonia. A final washing in water completes the purification. By these methods, the inorganic content of the fabric is reduced to about 0.02 per cent. An ash content lower than this is found to give poor results in the finished product. The moist fabric is dried in special ovens through which a hot-air draught passes.

The long cylinder of fabric is then placed in a special machine which cuts it into pieces of fixed length, ready for the next stage in the process.

\section{Impregnation, Fixing, and Branding.}

The "shaping" of the mantle must now be described. In the case of the ordinary upright mantle, this is done before 
impregnation; whilst the inverted mantle is shaped after that process is complete. The upright mantle is reinforced at the head by a piece of cotton or ramie tulle which is stitched on; and the head so formed is drawn together into the shape seen in the ordinary mantle. An asbestos hook is also attached. In the case of inverted mantles, the stitching is done with impregnated thread.

The process of impregnation is the main factor in producing a good mantle. It is found that mantles soaked in a solution of a pure thorium salt do not give results as good as those dipped in solutions containing cerium salts in addition to thorium. For example, if we take the intensity of the light given by a pure thorium mantle as 20 , the intensity shown by a mantle containing 99 per cent. thorium and 1 per cent. cerium is about 140. Further addition of cerium above 1 per cent. leads to a decrease in the illuminating power of the mantle. But this is not the only factor with which we are concerned. Since the mantle will be subjected to shocks, it is necessary to provide it with a skeleton capable of resisting vibration; so about 1 per cent. of other materials such as aluminium, calcium, zirconium, beryllium or magnesium is added to the impregnating solution.

The time of impregnation varies according to the material of the mantle; a few minutes suffices in the case of cotton or ramie, but artificial silk mantles require longer immersion. After the soaking is completed, the mantles are run between rollers which remove exactly the proper amount of moisture: or, in the case of artificial silk mantles, the excess solution is removed in a centrifuge.

The next stage in the process is termed "fixing." Consideration will show that the lower end of the upright mantle and the upper end of the inverted mantle are the weakest points in the structures; for it is at these places that the greatest strain will come in practice. The lower end of the upright mantle is exposed to draughts and hence is liable to fray; - whilst the upper end of the inverted mantle carries the weight of the whole device when in position. These two points are therefore reinforced by an additional treatment with a solution containing such materials as aluminium nitrate, borax, or calcium nitrate; and in this way extra 
mineral matter is deposited at the weak spots, thus forming a stronger skeleton.

Branding the maker's name upon the mantle is accomplished by taking advantage of the fact that "didymium" salts decrease the luminosity of the thorium mixture. A solution of " didymium" nitrate is employed for the inscription which becomes visible as dark lettering on the bright ground of the mantle when in use.

\section{Burning, Collodionizing, and Testing.}

The mantles are now ready for the last steps in the process. They are shaped on wooden models and are then subjected to the action of Bunsen burners which "burn off" all the original organic material from the mantle. The heating is begun at the closed ends of the mantles, as otherwise the contraction of the fibre under the heat would distort the shape of the fabric. This first stage in heating leaves the mantle a mere fragile structure; and in order to toughen it, high-pressure burners are brought in play which consolidate the more fusible oxides in the mantle and render the whole mass more resistant.

It is next necessary to provide some support for the ash skeleton so that it may stand the shocks of transport. This is provided by the collodionizing process. In groups of forty to sixty, the mantles are dipped into a mixture of nitrocellulose and various oils. The oils are added to reduce the rapidity of combustion of the nitro-cellulose. The dipping is done by machinery; and the mantles are then dried in ovens heated by high-pressure steam. When dry, they are trimmed in a machine and are then ready for packing.

Out of each batch, it is usual to test 1 or 2 per cent. of the mantles in order to see that they are up to standard. The test is applied by lighting them over a burner in the usual manner and then applying to the stand a series of shocks by means of a machine-driven hammer. The usual test for an upright mantle is 1000 shocks at the rate of 300 per minute from a $2 \mathrm{oz}$. hammer; whilst an inverted mantle is expected to remain in good condition after being submitted to 3000 shocks from a $3 \mathrm{oz}$. hammer at the rate of 600 shocks per minute. 


\section{CHAPTER XII}

\section{POSITIVE RAY ANALYSIS AND MASS SPECTRA}

\section{The Apparatus.}

IN the first section of Chapter VII., it was pointed out that when the cathode of a Crookes' tube is pierced with one or more apertures, a stream of "canal" or "positive" rays travels from the cathode in a direction opposite to that taken by the cathode rays. These positive rays are streams of the residual molecules of gas in the tube which have acquired a positive charge. Like the particles which make up the cathode stream, they can be deflected from their normal straight paths by the action of either magnetic or electric fields; and the deviation in each case is proportional to the ratio between the mass of the particle and the charge which it carries. Sir J. J. Thomson has utilized this property in devising a method for the recognition of the nature of these residual molecules; and his method has come to be known as positive ray analysis. ${ }^{1}$

In order to employ the positive rays as a means of qualitative gas analysis, three things are required : (1) an apparatus for generating positive rays ; (2) means of applying an electric and a magnetic field to the rays; and (3) a contrivance for registering the deviations thus produced. The essentials of the apparatus are shown in the diagram below (Fig. 16). A represents part of the glass flask which acts as the Crookes' tube. It has a capacity of from 1 to 2 litres. The cathode $\mathrm{C}$, of special construction, is placed in the neck, D, of the flask as shown; and protrudes into an ebonite box, UV. The joints

1 For a full account of the subject, see Thomson, Rays of Positive Electricity and their Application to Chemical Analysis (1913); a very clear description is given in Crowther's Molecular Physics; and a summary is to be found in Thomson's Bakerian Lecture, Proc. Roy. Soc., 1914, A, 89, 1. 
are made gas-tight with sealing-wax; and this is protected from heating effects by the water-jacket J. L and $M$ are pieces of soft iron which are used as poles for the electric field. They have plain faces which are about $3 \mathrm{~cm}$. long and they stand about $1.5 \mathrm{~mm}$. apart from each other. They are connected with the terminals of a battery of storage cells and in this way any required difference in potential can be maintained between them. $\mathrm{P}$ and $\mathrm{Q}$ are the poles of an electro-magnet. $F$ is a conical glass vessel about $40 \mathrm{~cm}$. long which is fastened to a camera containing the photographic plate on which the results are recorded. WV are iron plates which are interposed in order to screen the positive ray generator from the influence of the electro-magnet.

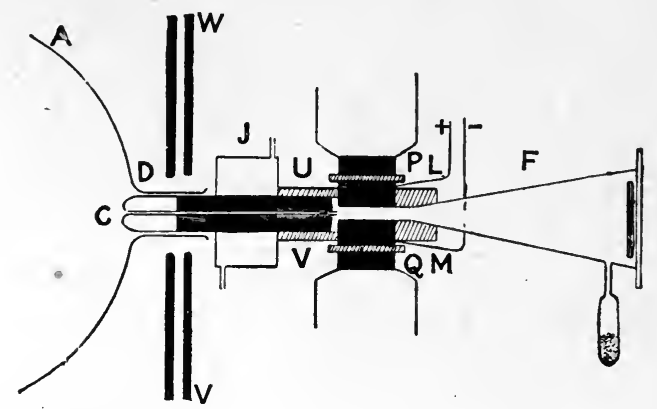

(From Sir J. J. Thomson's " Rays of Positive Electricity.")

Fig. 16.

The shape of the cathode, C, can be seen from the figure. The unshaded portion is made of aluminium; and the rest is composed of soft iron. Through the aperture down the centre passes a tube of copper with a bore of about $0.1 \mathrm{~mm}$. and a length of about $7 \mathrm{~cm}$.

One point of great importance must be mentioned. When a positively charged molecule enters the conical vessel $\mathbf{F}$, it may ionize the residual gas contained therein. The result of this will be that its positively charged fellows which follow it may find awaiting them negatively charged gas molecules; and discharges may occur between the two sets. As a consequence, the incoming particles lose their charges and cease to be acted on by the electric and magnetic fields; and complications are introduced. To avoid this, the pressure in the 
vessel $\mathrm{F}$ is kept low by absorbing the gas by means of charcoal and liquid air; and since the leak from the generating flask is small-on account of the narrowness of the connecting tube in the cathode-it is possible to maintain a difference in pressure between the generator $\mathrm{A}$ and the receiver $\mathrm{F}$.

\section{The Theory of the Method.}

Let us imagine that a residual molecule of gas becomes charged in the vicinity of the anode of the Crookes' tube which forms part of the positive ray apparatus. Such a particle will be driven towards the cathode by the electrical forces in the Crookes' tube; and in this process it will acquire an energy which may be represented by $\mathbf{D} \times e$, where $\mathrm{D}$ is the difference of potential between the electrodes and $e$ is the charge on the particle. The velocity of the particle when it reaches the cathode will therefore be $v$ in the equation-

$$
v^{2}=2 \mathrm{D} \times \frac{e}{m}
$$

where $m$ is the mass of the particle in question.

This particular value of $v$ represents a maximum velocity; for if the particle becomes charged at a point nearer the cathode, the driving force will have a shorter time to work upon it and hence its velocity will be lower than if it started in close proximity to the anode.

Now if neither the electro-magnet nor the static electrical field be in operation, the particle will pass through the cathode tube unaffected and will strike a point on the photographic plate directly opposite to the cathode aperture. Let us represent this point by $\mathrm{O}$ in the figure below.

Let us next assume that we apply the magnetic field; and that this tends to deviate the next particle from its straight path in the direction of $M$ in the figure. Instead of striking the plate at $O$, the new particle will fall at, say, the point $P$.

Further, let us imagine that the magnetic field is switched off and that the electric field is brought into operation instead. The deviation in this case will be at right angles to that produced by the magnetic field; and a particle under its influence will be deflected from $\mathrm{O}$ in the direction of $\mathrm{E}$. Suppose that it strikes the plate at $Q$. 
Obviously, if both fields are in operation at the same time, the particle will be deflected towards $\mathrm{M}$ and towards $\mathrm{E}$ simultaneously; and it will strike the plate at the point $R$. We have now to determine the position of $R$ in terms of the forces in operation.

Without going into details ${ }^{1}$ it may be said that if $M$ be the magnetic field, the particle will be deflected through a distance $k_{1} \cdot \frac{\mathrm{M} \cdot e}{m v}$, where $k_{1}$ is a constant depending on the apparatus; and if $\mathrm{E}$ be the electric field, the deviation due to it will be measured by $k_{2} \cdot \frac{\mathrm{E} . e}{m v^{2}}$. Or, in other words, calling the electric deflection $x$ and the magnetic one $y$, we have

$$
\begin{aligned}
& \text { Magnetic deflection }=\mathrm{OP}=\mathrm{QR}=k_{1} \cdot \frac{\mathrm{M} \cdot e}{m v}=y \\
& \text { Electric deflection }=\mathrm{OQ}=k_{2} \cdot \frac{\mathrm{E} \cdot e}{m v^{2}}=x
\end{aligned}
$$

From this it follows that

and

$$
\begin{gathered}
y / x=\left(\frac{k_{1} \mathrm{M}}{k_{2} \mathrm{E}}\right) v \\
y^{2} / x=\left(\frac{k_{1}^{2} \mathrm{M}^{2}}{k_{2} \mathrm{E}}\right) \frac{e}{m}
\end{gathered}
$$

Now if the magnetic and electric fields are not varied, it is clear that all the quantities within the brackets in the last two expressions are constant; and hence

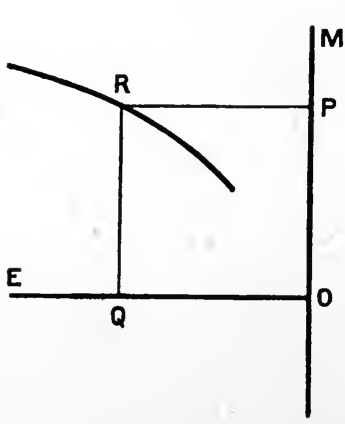

Frg. 17. $y / x$ forms a measure of the velocity of the deflected particles whilst $y^{2} / x$ gives the ratio of the mass to the charge on a particle.

So far, we have considered the case of a single particle; and our reasoning holds good for all identical particles, so that all such particles would strike the plate at the point $R$. In actual practice, however, as we have seen, the positive ray is made up of a series of particles which may be travelling at different velocities. The expression $y / x$ will have a different value for each particle; but if the particles all have the same

1 See Thomson, Rays of Positive Electricity, p. 7 (1913). 
. 


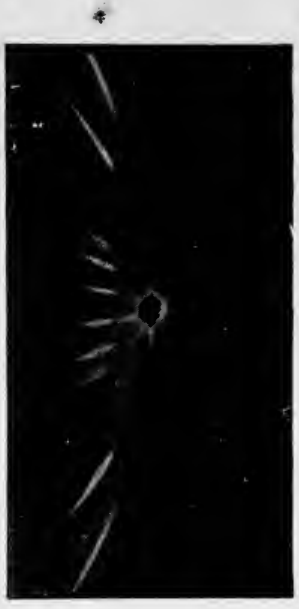

FIG. 18.

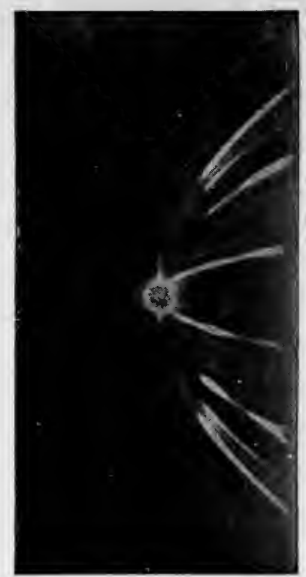

FIG. 19.

(From Sir J.J. Thomson's "Rays of Positive Electricity.") 
ratio of charge to mass, then the expression $y^{2} / x$ will be constant for the whole series. In other words, these particles will strike the plate at a series of points which will lie on some curve which can be expressed by $y^{2} / x=$ constant. This curve is obviously a parabola.

Let us now put this into a more concrete form. Imagine that one particle is a helium atom and that another is an atom of argon. Let us suppose that each carries a unit charge. Then the helium atom will have a ratio of mass to charge equal to $4: 1$, whilst the argon atom will have the ratio $40: 1$. Thus the helium atom will strike the plate somewhere on a curve represented by $y^{2} / x=4 k$; and the argon atom will strike the plate somewhere on a curve for which $y^{2} / x=40 k$. Thus the two atoms will be sifted away from each other on the plate. Further, if we imagine a stream of helium atoms moving at different velocities in the ray, they will distribute themselves along the parabola $y^{2} / x=4 k$; and a similar stream of argon atoms will distribute themselves over the parabola $y^{2} / x=40 k$.

As will be seen in the next section, the dimensions of the parabolas enable us to calculate the ratio of the charge to the mass for those molecules which leave traces upon the photographic plate.

\section{The Interpretation of the Photographs.}

The illustrations (Figs. 18 and 19) give some idea of the results obtained by the use of Thomson's apparatus.*

In the first place, it will be noticed that both photographs contain a bright spot at the apex of the parabola. This spot is the point of impact of the undeviated rays which pass when the electric and magnetic fields are not in operation. Secondly, it will be seen that two branches of the parabolas are shown, one above and the other below the apex. These two branches are produced by taking two separate exposures in both of which the electric field is kept constant: in the one exposure

* In the latest form of the instrument a sheet of metal is placed over the photographic film and this sheet has a fine parabolic slit cut in it. By varying the magnetic field and keeping the electric field constant, the parabolas of the various constituents of a mixture under analysis can be successively brought into coincidence with the slit. This avoids the "splashed" appearance shown in the photographs given in Figs. 18 and 19. 
the magnetic field operates in one direction; whilst in the second exposure it is reversed: so that the particles are diverted in opposite directions (up the plate in one case and down it in the second instance). The parabolic form of the curves is easily recognizable in the illustrations. It must be strongly emphasized that these tracks are not formed by the particles grazing along the plate; but that they represent a series of "bullet-marks" made by particles striking the plate from above, the separate "marks" being so close together that they merge into lines.

It will be noticed that the parabolas stop short and are not continued to the apex. The reason for this is obvious. In order that any particle should strike

$I^{m}$ the plate in close proximity to the apex, the amount of deviation undergone by it would need to be small; and hence its velocity would need to be very great. But there is a limit to the velocity of the particles owing to the limited difference in potential between the electrodes of the Crookes' tube; and thus it comes about that we do not find particles travelling with a velocity higher than a certain maximum. This maximum velocity is sufficient to bring them within a certain distance of the apex, but it is not sufficient to over-step this point; so that no particles strike the plate in the neighbourbood of the apex.

The method of measuring the plates can be seen from the diagram above.

Draw any line AF parallel to OM and cutting to two parabolas at $\mathrm{A}, \mathrm{B}, \mathrm{D}$, and $\mathrm{F}$ and meeting the line $\mathrm{OE}$ at $\mathrm{C}$. The value of $y_{1}$ (for the outermost parabola) is equal to $\mathrm{CA}$; the value $y_{2}$ (for the innermost parabola) is equal to $\mathrm{CB}$. In both cases the value of $x$ is the same, being equal to OC. Now from the equations in the last section the value of the fraction $\frac{\text { charge }}{\text { mass }}$ for any particle is given by $y^{2} / x$. Therefore, if $m_{1}$ and $e_{1}$ be the mass and charge for particles on the outermost 
parabola and $m_{2}$ and $e_{2}$ be the corresponding factors for particles on the second parabola, we have-

$$
\mathrm{AC}^{2} \div \mathrm{BC}^{2}=\mathrm{y}_{1}^{2} \div \mathrm{y}_{2}^{2}=\frac{y_{1}^{2}}{x} \div \frac{y_{2}^{2}}{x}=\frac{e_{1}}{m_{1}} \div \frac{e_{2}}{m_{2}}=\frac{m_{2} e_{1}}{m_{1} e_{2}}
$$

and if $e_{1}=e_{2}$ then $\quad \frac{\mathrm{AC}^{2}}{\mathrm{BC}^{2}}=\frac{m_{2}}{m_{1}}$

Thus if all the particles carry the same charge, the ratio of the masses of the two sets of particles is given by the ratio $\mathrm{AC}^{2}: \mathrm{BC}^{2}$, or from the more easily measurable ratio $\mathrm{AF}^{2}: \mathrm{BD}^{2}$, since $\mathrm{AF}=2 \mathrm{AC}$ and $\mathrm{BD}=2 \mathrm{BC}$. In actual practice it is found that this condition is fulfilled in most cases; and when any deviation occurs owing to a particle carrying more than one charge the matter is not serious, for the number of charges carried by any particle is always small.

The following table ${ }^{1}$ gives an idea of the manner in which the results obtained from the photographs are interpreted. The first column gives the distance $d$, of the different parabolas from the axis $\mathrm{OE}$, measured along a common ordinate. The second column gives the ratio of mass to charge, assuming that all the particles carry the same charge. The third column gives an interpretation of this when modified by the assumption of varying charges.

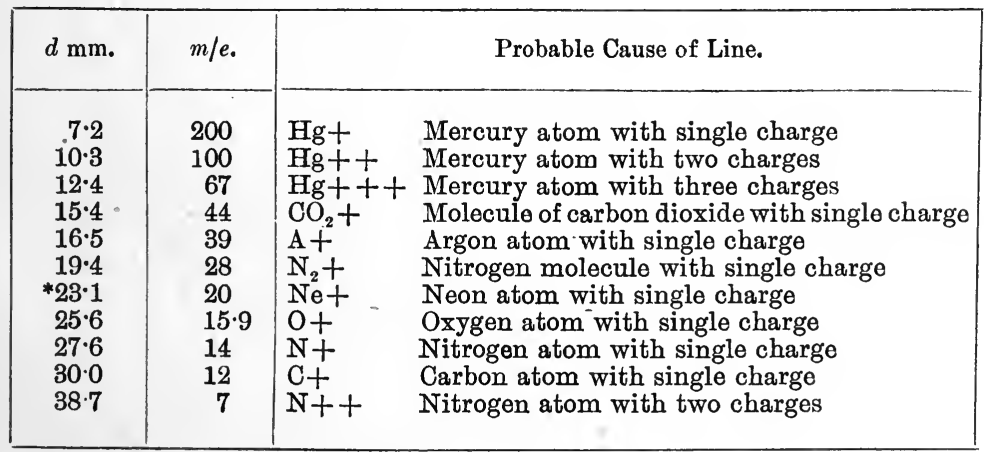

For instance, the heaviest atom likely to exist in the gas under examination is a mercury one with an atomic weight of 200. Since the ratio of the mass to charge is $200: 1$, it is clear that this atom carries a single charge. Again, another parabola corresponds to the ratio of mass to charge $100: 1$. This

1 Crowther, Molecular Physics, p. 52 (1914). 
would represent an element of atomic weight of 100 with a single charge on the atom or an element of atomic weight of 200 with two charges on the atom. We have therefore to choose between the probability that the line is produced by a mercury atom carrying two charges or by an atom of, say, ruthenium, with one charge. Obviously the mercury hypothesis is the more probable one. Then again, in the case of the third line, the atomic weight of the element must be approximately 67 or 134 or 201. Neither zinc nor caesium is likely to be present under the experimental conditions, so we assume that the " 67 " line is due to an atom of mercury carrying three charges.

The only point about which there might be a doubt in the above interpretation is in the case marked with an asterisk; for the value 20 might be obtained either from a neon atom with one charge or from an argon atom with two charges. The chance of its being due to a mercury atom with ten charges is ruled out by the fact that it is rare that mercury carries even as many as eight charges, to judge from the general run of results.

From the above it will be clear that in the stream of positive rays we may have: (1) positively electrified atoms with one charge; (2) positively electrified molecules with one charge; and (3) positively electrified atoms with multiple charges. In addition to these we may also find tracks due to negatively electrified atoms and molecules which have become neutralized on passing through the cathode and have picked up a cathode particle en route. We need not, however, enter into this question here. ' From the chemical point of view it is interesting to note that among the carbon compounds the negatively charged molecule is not detected in the case of methane, carbon dioxide, phosgene, etc., wherein carbon is linked only to other elements; but negatively charged molecules are found when the compound examined contains two directly linked carbon atoms as in the cases of acetylene, ethylene, or ethane. In the examination of benzene vapour, negatively electrified triplets of carbon atoms were observed, and there seems to be a possibility that quartets may have been present.

1 Thomson, Proc, Roy Soc., 1914, A, 89, 1. 


\section{The Substance $\mathrm{X}_{3}$.}

When solids are bombarded with cathode rays, various gases seem to be given off; and the examination of these by the positive ray method yielded facts of some interest. The results point to the presence in the gas mixture of neon, helium, and a third substance to which the name $X_{3}$ has been given.

With regard to the sources of $\mathrm{X}_{3}$, it has been found that it is produced by the action of cathode rays upon a very varied series of substances. Platinum, palladium, aluminium, copper, zinc, iron, nickel, silver, gold, lead, graphite, diamond dust, lithium chloride, and other metallic salts as well as some meteorites have been found to liberate the gas. The presence of mercury vapour in the bombardment tube diminishes the intensity of the line due to $X_{3}$; from which Thomson deduces that $\mathrm{X}_{3}$ combines with mercury vapour under the influence of the electric discharge.

If we assume that the maximum number of charges which can be carried by a particle is limited, there appear to be only two possible explanations for the $\mathrm{X}_{3}$ line. It must be produced by something in which the ratio of mass to charge is three times that found in the case of a hydrogen atom. This can be accounted for by assuming that $X_{3}$ is either (1) a carbon atom carrying four electrical charges; or (2) a molecule containing three hydrogen atoms and carrying a unit charge. Let us examine these possibilities in turn.

In the first place, the gas giving the " 3 " line can be stored in the bombardment vessel for days and yet it will produce the characteristic line when analysed after this period. If the line be due to carbon atoms carrying four charges, it must be because some volatile carbon compound is produced during the bombardment which, when introduced into the discharge tube gives, on the passage of the discharge through it, carbon atoms with four charges. Experiments have been made by introducing directly into the discharge tube such substances as methane, carbon dioxide, carbon monoxide, ethylene, ethane, acetylene, carbonyl chloride, and carbon tetrachloride; but none of these gives results $*$ analogous to $\mathrm{X}_{3}$. Other evidence

* $C f$., however, Collie and H. S. Patterson's observation that carbon compounds are retained under conditions which might normally be expected to preclude their appearance (Proc., 1913, 29, 217). 
is furnished by the nature of the tracks on the plate, but into this we need not enter here. ${ }^{1}$

With regard to the possibility that $\mathrm{X}_{3}$ is a singly-charged molecule containing three hydrogen atoms, we have the following evidence. Whenever large amounts of $\mathrm{X}_{3}$ are produced, spectroscopic examination detects the presence of a considerable quantity of hydrogen in the gas liberated by the cathode bombardment. When various salts were bombarded after recrystallization, it was found that those like potassium iodide, lithium carbonate, and potassium chloride gave smaller yields of $\mathrm{X}_{3}$ after recrystallization; whereas in the case of potassium hydroxide, lithium hydroxide, lithium chloride, and calcium chloride recrystallization had no effect upon the output of $\mathrm{X}_{3}$. It will be noticed that the first class have no hydrogen in their composition; whereas the second group either contain hydrogen or are deliquescent and thus absorb hydrogen in watermolecules: This is regarded by Thomson as a proof of the connection between hydrogen and the $\mathrm{X}_{3}$ line.

Turning now to the properties of $\mathrm{X}_{3}$ as far as they are known, the following seem important: $X_{3}$ when mixed with hydrogen, is not markedly photo-sensitive; but if a mixture of $\mathrm{X}_{3}$ and oxygen be exposed to a magnesium flash, the $\mathrm{X}_{3}$ is destroyed. The passage of an electric spark has an analogous effect: hydrogen and $\mathrm{X}_{3}$ will stand a good deal of sparking without any noticeable result; but if a spark be passed through a mixture of $X_{3}$, oxygen and hydrogen, the $X_{3}$ disappears. In the absence of oxygen, $X_{3}$ can be heated to a high temperature without destruction; but it disappears when raised to a redheat in presence of copper oxide. Like hydrogen, $\mathrm{X}_{3}$ has the power of passing through a palladium wall; but its rate of transfusion is much lower than that of hydrogen.

On these grounds, Thomson assumes that $\mathrm{X}_{\mathbf{3}}$ is really a triatomic molecule of hydrogen, $\mathrm{H}_{3}$; and he considers it to be the hydrogen analogue of ozone. It is evidently more stable than ozone, as is seen from its resistance to high temperatures. No particular spectrum has been observed for $\mathrm{X}_{\mathbf{3}}$; for a mixture of it and hydrogen exhibits only the normal hydrogen spectrum.

Against this may be urged the evidence brought to light

1 See Thomson, Rays of Positive Electricity, p. 117 (1913). 
by Collie and H. S. Patterson in the course of work in a different field. ${ }^{1}$ They found that when a heavy discharge is passed through a vacuum tube, quite considerable quantities of hydrogen can be made to disappear. For example, in one experiment, as much as 3.6 c.c. of hydrogen apparently vanished. A gas is produced ${ }^{*}$ which gives a carbon spectrum; and this gas, like $\mathrm{X}_{3}$, disappears when sparked with mercury vapour. Further, it is not easily condensed by the use of liquid air.

It is a well-known fact that certain carbon compounds are extremely difficult to remove from the surface of glass; and a reference to the description of Thomson's apparatus which was given above will suffice to recall the fact that parts of it are stuck together with sealing-wax which would furnish a source of abundant carbon. Tap-grease also might yield certain carbon derivatives. In the case of ordinary measurements, factors like these might be disregarded; but the extraordinary sensitiveness claimed for the positive ray method unfortunately renders it all the more liable to difficulties arising from such minor sources of impurities.

The fairest course in the matter appears to be to regard the problem of $\mathrm{X}_{3}$ as still unsolved. It may be merely a carbon atom carrying four electrical charges; or it may be an allotropic modification of hydrogen. Some of the evidence points in one direction, some in another. It is too early yet to decide definitely in favour of either hypothesis.

Some work has been carried out which appears to throw further light upon the nature of $\mathrm{X}_{3}$. Duane and Wendt ${ }^{2}$ found that when hydrogen is exposed to the bombardment of $\boldsymbol{a}$-particles from niton, a contraction is observed. Part of this contraction is possibly due to the hydrogen being driven into the glass of the containing vessel. The remaining hydrogen appears to acquire properties differentiating it from the ordinary form of the element. It reduces sulphur to hydrogen sulphide, phosphorus to phosphine, arsenic to arsine and in a neutral solution of potassium permanganate it throws down manganese

1 Collie and H. S. Patterson, Proc., 1913, 29, 217.

${ }^{2}$ Duane and Wendt, Phys. Rev., 1917, 10, 116; Lindt, J. Amer. Chem. Soc., 1919, 41, 545.

* This gas was trapped in a jacket surrounding the discharged tube; and it may have originated from molecules driven through the inner tube by the force of the discharge. 
dioxide. It is unstable and reverts in about one minute to the ordinary bi-atomic hydrogen. The activity of this new variety of hydrogen is not due to the formation of charged hydrogen ions, as the application of a strong electrostatic field seems to be insufficient to remove the active variety, which it would do were ions of hydrogen the only factors concerned.

Wendt and Landauer ${ }^{1}$ have examined the matter further. They find that by discharging an induction coil through a tube of hydrogen under 2-8 cms. pressure the same active form of hydrogen can be produced. The new gas is unaffected by passage through spirals immersed in boiling water or in a freezing mixture composed of ice and calcium chloride; but it appears to be condensed by cooling with liquid air. The active form was also prepared by passing hydrogen through an ozonizer fed with a current of 20,000 volts. The gas formed in this manner combined directly with nitrogen to give ammonia. Attempts to prepare the active form of hydrogen by an application of the Schumann light were not successful.

It is suggested-as yet there is no proof-that the new form of hydrogen is triatomic and may correspond to Thomson's $\mathrm{X}_{3}$.

\section{- 5. Conclusion.}

It may be desirable at this point to sum up the advantages offered by the positive ray method of analysis. In the first place, it permits the analysis of extremely small quantities of a gas mixture. Thus a quantity of helium which does not exceed $4 \times 10^{-6}$ c.c. can be detected in a c.c. of air.

Secondly, it carries our knowledge further than spectrum analysis can do. If we have only a trace of an element mixed with large quantities of other gases, the spectrum of the mixture may fail to reveal the presence of the trace owing to its characteristic lines being swamped by the spectra of its companions; whereas in the case of the positive ray method, each constituent is sifted out from the others.

Thirdly, in the case of a new element, an examination of the spectrum tells us only that new lines are present; but with positive ray analysis it may be possible to go much

1 Wendt and Landauer, J. Amer. Chem. Soc., 1920, 42, 930. 
further. For example, if we find two parabolas characteristic of the new element, one must arise from the atom and the other from the diatomic molecule. If the substance is monatomic, there will be only one parabola, or, if there be more than one produced (owing to the atom taking up more than a single charge), we can detect the nature of the charged body.

These facts prove that in the positive ray method we have gained a new and formidable weapon for the chemical armoury; and since the subject is still new, many further developments may yet be looked for in its application to chemical problems. In a later chapter* some of these will be examined.

* See Chapter XVII. 


\section{CHAPTER XIII}

\section{THE PHENOMENA OF RADIOACTIVITY}

\section{Historical.}

IN 1895 Röntgen showed that when cathode rays impinged upon the end of a vacuum tube they gave rise to a green luminescent patch, from which was projected a series of rays - the X-rays. From the phosphorescence of the Crookes' tube to the green phosphorescence of certain minerals is only a short step, and in 1896 Becquerel began an investigation of the latter phenomenon. He found that crystals of potassium uranium sulphate had the property of affecting a sensitive photographic plate (wrapped in black paper) in exactly the same way as it would have been affected by an X-ray discharge. The experiment led to the discovery of what are now called the Becquerel rays.

These radiations are invisible to the eye, just as the $\mathrm{X}$-rays are. They are given off by metallic uranium, and also by uranium salts. Like the X-rays, they can pass through thin sheets of glass or metal; and a further resemblance is to be found in the fact that neither set of rays can be refracted by ordinary means. Again, the Röntgen rays have the property of ionizing gases through which they are passed; and it has been found that in this instance also, the Becquerel rays resemble the others, though their action is much more feeble. If we charge the leaves of a well-insulated electroscope they will remain separated for a very considerable time, owing to the fact that ordinary dry air is a poor conductor of electricity ; but if we bring a piece of uranium near the electroscope, the Becquerel rays which are given off from the metal at once ionize the air, making it a better conductor of electricity, and thus 
the leaves of the electroscope fall together much more rapidly than they did before the uranium was brought near them. We thus have a method of determining the activity of any particular sample of uranium; for we need only measure the rapidity with which an electroscope is discharged when the sample is placed in its vicinity. If the uranium is sending out many Becquerel rays, the electroscope leaves will soon fall together; if the Becquerel rays are few, the instrument will be very slowly discharged.

It would naturally be concluded, from the above evidence, that the Becquerel rays and the Röntgen rays were identical. As a matter of fact, however, they are not so. We need not go into details here, as the matter will be dealt with in a later section.

Soon after Becquerel's work on uranium and its salts, Schmidt discovered that thorium also had radioactive properties; and since that time various other elements have been described which belong to the same class. Of these the most important is radium.

We need not continue the history of the subject further in this section. The present chapter will be devoted to a discussion of the various radioactive metals. In the following chapter, some account will be given of the changes which these bodies spontaneously undergo. The chapter after that will contain a description of the gases which are evolved during radioactive change. Of course, in the space at our disposal, no attempt can be made to treat the subject in great detail. The properties of radium and its derivatives will be taken as typical and described at some length, while the allied substances will be dealt with very briefly.

\section{Radium.}

In this and the succeeding sections we shall attempt to give an outline of the properties of the various radioactive substances. To enter fully into the physical side of the question would carry us beyond the limits of the present volume : so, as far as possible, the subject will be treated from the point of view of chemistry, and the physical part of the subject will be 
dealt with only in so far as it aids the comprehension of the purely chemical side. ${ }^{1}$

In the introductory section of this chapter, attention was called to the fact that uranium salts throw off radiations which affect photographic plates just as the X-rays do. Now, uranium compounds are found naturally in the mineral pitchblende, which occurs usually (like the rare earths) in igneous rocks such as granite. Madame Curie, ${ }^{2}$ in the course of an examination of a great number of naturally occurring substances, discovered that in some cases the natural ore was much more radioactive than the amount of uranium salt contained in it would lead us to expect. For example, pitchblendes are about four times as active as metallic uranium; chalcolite (a double phosphate of uranium and copper) is twice as active as uranium. Further, when Madame Curie prepared artificial chalcolite, she found that instead of being more active than metallic uranium, it was two and a half times less active. This proves conclusively that the activity of the natural chalcolite was not due entirely to the uranium contained in it, but must be attributed to the presence in the natural ore of some substance not found in the synthetic product.

The presence of this new body in pitchblende and other minerals having been established, the question of extraction then arose. It was easy to carry out an ordinary analysis, and to determine by measurements with an electroscope whether the activity was a property of the filtrate or of the precipitate. We need not enter into any details with regard to the method of analysis employed. ${ }^{3}$ A series of operations must be gone through before we can obtain a mixture consisting of radium bromide and barium bromide.

After the isolation of the bromides of radium and barium from the mixture has been accomplished, it is necessary to separate them from each other. This is done by fractional

1 For an account of the practical methods employed in radioactivity measurements the reader is referred to Makower and Geiger's Practical Measurements in Radioactivity.

2 Thesis presented to the Faculté des Sciences, Paris; see Chem. News, 1903, 88, 85, 97, 134, 145, 159, 169, 175, 187, 199, 211, 223, 235, 247, 259, 271.

3 Ebler and Bender, Zeitsch. anorgan. Chem., 1914, 88, 255 ; U.S.A. Bureau of Mines Bulletin 104 (1915); Schlundt, J. Physical Chem., 1916, 20, 485. 
crystallization, either from aqueous solution or from a solution of the salts in water acidified with hydrobromic acid. The latter is the better method of the two. It is possible to employ the method of fractional precipitation instead of crystallization, the mixed salts being thrown down from an aqueous solution by means of alcohol. In either case, the radium bromide is less soluble than the barium-salt.

The radium salts which are obtained in this way resemble the corresponding barium compounds in many respects. Radium chloride is isomorphous with barium chloride; and the two salts, when they are freshly prepared, are similar to each other in appearance. It has been found that if the radium salt stands for a time it gradually becomes coloured, the tint ranging from yellow to rose-pink. The coloration becomes much more marked if a trace of impurity (such as a barium salt) be present in the crystals.

Another property which is noticeably altered by lapse of time is the blue luminescence exhibited by freshly prepared radium salts or their solutions. The presence of barium salts in this case also appears to have some influence, for the blue light is more strongly exhibited by an impure sample of radium salt than it is by a pure one.

An aqueous solution of a radium salt has been found to evolve a mixture of oxygen and hydrogen, and there appears to be no cessation of this process, which offers such a peculiar parallel to electrolytic action. ${ }^{1}$ A still more extraordinary property of these salts, however, remains to be described. Curie and Laborde ${ }^{2}$ observed that the temperature of a radium salt is always a little higher than the temperature of the air about it; in other words, the radium salts are continually giving out heat. Curie and Laborde showed that the quantity of heat disengaged in one hour by one gramme of pure radium would amount to about a hundred gramme-calories.

When a radium salt is placed in the flame of a Bunsen burner it gives rise to an intense carmine-red coloration, which is very characteristic. The spectrum of the element has been examined by several workers, and found to be quite different from any known spectrum.

\footnotetext{
1 See also Ramsay, Monatsh., 1908, 29, 1013.

2 Curie and Laborde, Compt. rend., 1903, 136, 673.
} 
The atomic weight of radium was at one time the subject of considerable controversy, ${ }^{1}$ but it is now taken as 226.0. This value places radium below barium in the alkaline earth column of the Periodic Table; and the resemblance between the two elements fully justifies the position.

Metallic radium has been isolated by Madame Curie and Debierne. $^{2}$ A solution containing about a decigramme of radium chloride was electrolysed, the cathode being mercury and the anode being platinum-iridium. After the electrolysis was completed, the amalgam of radium at the cathode was placed in an iron boat and heated in a current of hydrogen which had previously been purified by passage through the walls of a platinum tube heated in an electric furnace. At approximately $700^{\circ} \mathrm{C}$., the mercury had all been driven off and the boat contained a brilliant white metal which fused sharply in the neighbourhood of $700^{\circ} \mathrm{C}$. and began to attack the quartz tube in which the experiment was carried out. On exposure to air, a black film formed upon the metal, this being probably radium nitride. Radium attacks water violently, forming a . soluble oxide. A trace of the metal having fallen upon a sheet of paper, it was found that the paper was blackened and apparently carbonized. It appears that metallic radium is more volatile than barium.

\section{The Becquerel Rays.}

In the previous section we mentioned that the salts of radium and other radioactive elements emitted a series of radiations which have been termed the Becquerel rays, from the name of their discoverer. Since a very considerable part of the peculiar activity of radioactive bodies is closely connected with these rays, it is necessary at this point to enter into some consideration of the question. The first problem which we have to solve is that of the nature of the rays in question. Are they simple, or are they made up of a series of different types of vibration, such as a mixture of light and X-rays?

1 Madame Curie, Chem. News, 1903, 88, 159; Compt. rend., 1907, 145, 422; Runge and Precht, Physikal. Zeitsch., 1908, 4, 285 ; Watts, Phil. Mag., 1903, 6, 64 ; Thorpe, Proc. Roy. Soc., 1908, A, 80, 298; Hönigschmid, Monatsh., 1912, 33, 253; Whytlaw-Gray and Ramsay, Proc. Roy. Soc., 1912, A, 86, 270.

2 Madame Curie and Debierne, Compt. rend., 1910, 151, 523. 
To settle this point, we have two methods at our disposal. In the first place, we may interpose in the path of the rays some sort of filter which will give us a chance of separating one component from the rest; or, secondly, we may pass the rays through a magnetic field, and determine whether they are uniformly deflected as a whole or whether they can be resolved into a series of vibrations having different deflections.

We need not enter into any great detail with regard to the first of these methods. An experiment mentioned by Strutt ${ }^{1}$ will make the application clear. Suppose that we charge an electroscope so that the leaves of it diverge from one another. If we now bring near the electroscope a small quantity of a radium salt, we shall find that the leaves fall together, say, in ten seconds. Let us next wrap the radium salt in a sheet of tin-foil and again place it in position near the electroscope ; we shall find that the leaves take longer to fall, say, a hundred seconds. We have thus filtered off some part of the rays. Now, if all the rays were of the same type, we should expect to increase the time of discharge to a thousand seconds by doubling the sheet of tin-foil. This, however, is not found to be the case; but, instead, the rate of discharge hardly falls at all with the superposition of the second thickness of tin-foil. It is thus made clear that we have filtered off one set of rays by means of the tin-foil, but that there still remain other rays which the tin-foil sheets do not arrest. We have in this way established the presence of two types of vibration, one set stopped by tin-foil, the second not. We have now to discover whether or not we can further sift the rays to which tin-foil is transparent. To do this, we substitute for the tin-foil a thin casing of lead; and here we find a similar behaviour. The addition of one sheet of lead causes a perceptible lengthening of the period of the electroscope's discharge; but the interposition of a second slip of lead does not further diminish the velocity of the discharge to any marked extent. Thus again we have reached a border-line between two sets of rays, one of which will pass through the lead, while the other cannot do so.

This method, however, is only a very rough one, and does not lend itself to measurements of such great accuracy as are obtainable by the second method, in which a magnetic field

1 Strutt, The Becquerel Rays and the Properties of Radium, 1904, p. 51. 
is used. The actual details of the experimental methods employed need not be described in this place, ${ }^{1}$ but we may give the results which have been obtained by the application of the electro-magnet to the problem.

Suppose that we have a magnetic field applied at right angles to the plane of the paper and directed towards the paper; the state of affairs may be represented by Fig. $21 .^{2}$

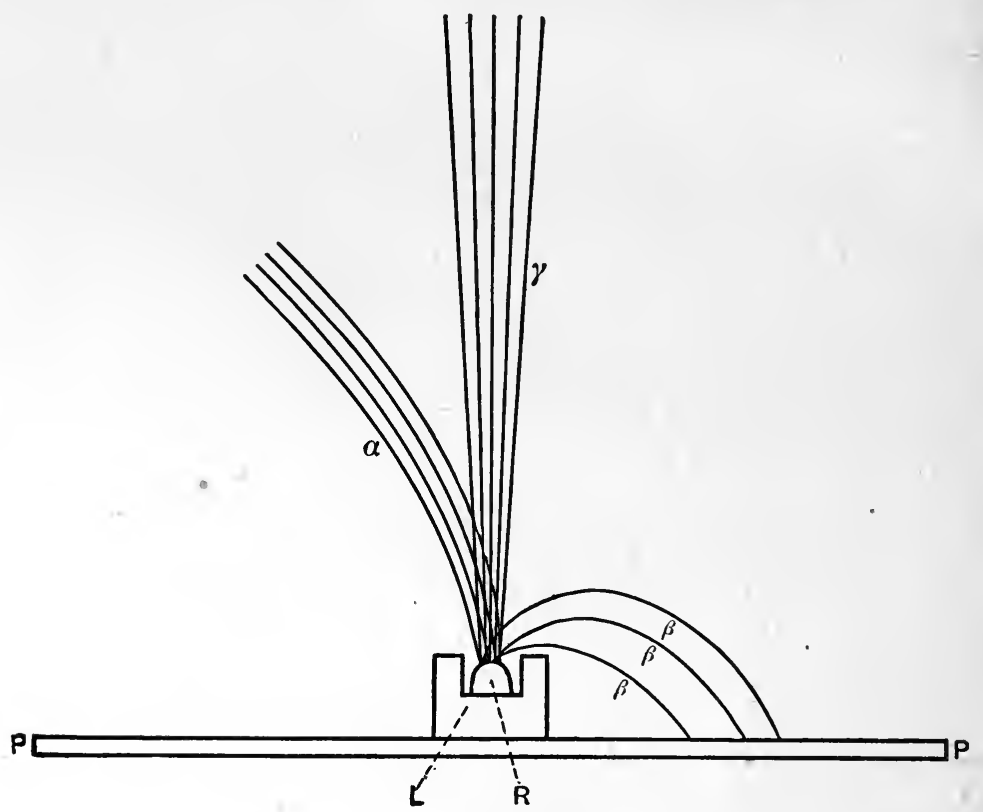

FIG. 21.

Here $\mathrm{L}$ is a piece of lead in which a hollow has been drilled. At the bottom of the hollow is placed some radium salt, $R$. Under the influence of the magnetic field, the radiations from the radium salt are split up into three groups, which have been termed by Rutherford the $\alpha-, \beta$-, and $\gamma$-rays.* The $\gamma$-rays are not deflected by the magnetic field, and are thus comparable to ordinary $\mathrm{X}$-rays ; but their penetrating power is very much

1 See Rutherford, Radioactivity, 1905, chaps. iv. and v.

2 Madame Curie, Chem. News, 1903, 88, 169.

* As will be seen later, the $\beta$-rays do not arise from radium itself but come from certain other substances which appear in specimens of radium salts after standing. 
greater than that of the $X$-rays. The $\beta$-rays resemble the cathode rays of a high vacuum tube; and like these they are deflected by the magnetic field in the manner shown in the figure. They appear to be streams of particles carrying a negative electric charge. If we place a sensitive photographic plate, $\mathrm{PP}$, under the leaden cell containing the radium salt, we shall find that the spots where the $\beta$-rays fall are affected by them. Finally, we come to the third set of components of the Becquerel rays. These, the $a$-rays, are deflected from the straight path of the discharge by the electric field; but instead of being bent in the same way as the $\beta$-rays, they are curved in the opposite direction. They are therefore positively charged particles and are now known to be helium atoms. We must now discuss the properties of these different rays in turn.

The $\gamma$-rays, as we have mentioned, are not deflected to any extent by a magnetic field, even when this is very powerful. Their penetrating power also is very marked. For instance, Strutt ${ }^{1}$ has observed that even 8 centimetres of sheet lead will not suffice to arrest these radiations. The origin of the $\gamma$-rays can easily be surmised. They resemble X-rays so closely as to leave little doubt as to the identity of the two types $;^{2}$ and bearing in mind the fact that the $\beta$-rays of radium and the cathode rays of a Crookes' tube are very closely allied, we must conclude that the $\gamma$-rays of radium salts are produced by the bombardment of the solid salt by the particles of the $\beta$-rays produced within itself.

The nature of the $\beta$-rays seems to be beyond doubt. They are material particles projected from the surface of the radium salt, just as particles are driven out from the cathode of a high vacuum tube. Naturally, they do not all travel with the same velocity; some sets of particles move more slowly than others, and it has been found possible to analyse the swarm of particles by deflecting them in magnetic fields. It has been found that the slowest-moving particles are most deflectedas can be foreseen-and it has been proved, further, that the $\beta$-rays as a whole are less deflected than the cathode rays from Crookes' tubes. Hence it is clear that the particles forming

1 Strutt, The Becquerel Rays, p. 83.

2 J. A. Gray, Proc. Roy. Soc., 1912, A, 87, 489. 
the $\beta$-rays of radium are moving at a much higher velocity than those which are shot out by the cathodes of vacuum tubes. It has been estimated that the velocities of light, the $\beta$-rays, and the ordinary cathode rays are approximately the following :-

\begin{tabular}{|c|c|c|}
\hline & - $30 \times 10^{4}$ & kilometres per seco \\
\hline de rays & $\begin{array}{ll}\text { - } & \left(6 \times 10^{4}\right) \text { to }\left(28 \times 10^{4}\right) \\
\text {. } & \left(2 \times 10^{4}\right) \text { to }\left(10 \times 10^{4}\right)\end{array}$ & $"$ \\
\hline
\end{tabular}

It appears, then, that the velocity of the electrons in the $\beta$-rays is greater than that of any other known natural body. From the fact that the radioactive bodies are losing negative electricity at a considerable rate owing to the departure of the electrons of the $\beta$-rays, it is clear that the remaining portion of the salt must gradually acquire a positive charge, and that if loss of this charge be prevented by insulation it will eventually become quite measurable. In point of fact, one experimenter $^{1}$ noticed that when a sealed glass tube containing radium was opened after several months, a bright electric spark was produced.

Turning now to the a-rays, we enter quite a different field. Here we have to deal with a series of helium atoms, travelling at high velocities (probably one-tenth that of light). These atoms are positively charged, and are deflected by magnetic fields in the direction opposite to that taken by the $\beta$-particles. The extent of the deflection, however, is by no means so great as that observed in the case of the electrons of the $\beta$-rays. The a-rays appear to be similar in character to the positive rays which can be produced in vacuum tubes. It seems certain that the heat generated by radium is due to the impacts of these particles. Crookes has devised an instrument, the spinthariscope, by means of which we can actually observe the effects of the collision between $a$-particles and ordinary matter. The spinthariscope consists of a zinc sulphide screen, above which a tiny fragment of a radioactive substance is suspended on the end of a steel pointer. When the screen is examined through a lens, it is found to be covered with tiny points of light which appear and vanish almost at once. These light-spots are apparently due to the phenomenon known as triboluminescence, 
in which the breaking of a crystal causes a flash of light; and it is supposed that the flashes in the spinthariscope are caused by the fracture of the zinc sulphide crystals under the impact of helium atoms discharged from the radioactive substance on the pointer. The triboluminescence phenomena on a larger scale can be observed by any one who rubs two pieces of sugar together in the dark.

In the foregoing paragraphs we have discussed the properties of the $a-, \beta$-, and $\gamma$-rays seriatim; but we must bear in mind that the effects produced by radioactive substances in general are not caused by the separate action of each type or ray, but are really due to the united action of all three kinds. We shall now give a few instances of the changes which radium produces upon various substances when it is placed in their vicinity.

The first effects which are recoguizable are those due to fluorescence or luminescence of one kind or another. Suppose that we bring an X-ray fluorescent screen near a radium compound; the screen will become strongly lit up, just as it is when acted upon by the Röntgen rays. Bary ${ }^{1}$ has found that many of the alkali metals and alkaline earths are also fluorescent when brought into the neighbourhood of a radium preparation. Madame Curie ${ }^{2}$ showed that paper, cotton, and other substances could exhibit the same phenomenon. Becquerel $^{3}$ observed that while a ruby will fluoresce under the action of light-rays, it remains inert with regard to radium. Again, a diamond which shines in presence of the rays from radium does not light up when $\mathrm{X}$-rays are thrown upon it; and calcium sulphide appears to behave similarly. Many other instances of this action of the Becquerel rays might be quoted.

If we expose a fluorescent substance of the type mentioned above to the continued action of the radium rays, it is found that the power of shining which it possesses does not remain constant, but slowly diminishes while at the same time the physical appearance of the fluorescent body changes. Barium platinocyanide on long exposure to the rays grows darker in colour, finally becoming quite brown; its luminescence also dies away gradually. Both the original colour and the power

1 Bary, Compt. rend., 1900, 130, 776.

2 Madame Curie, Chem. News, 1903, 88, 212.

3 Becquerel, Compt. rend., 1899, 129, 912. 
of fluorescing can be regenerated by exposing the salt to light for a time.

When some varieties of glass are exposed to the action of the Becquerel rays they become fluorescent, and at the same time become tinted brown or violet according to the alkali metal contained in them. If we warm the glass after the process has gone on for a time, we shall find that it loses its tint and becomes again transparent, regaining at the same time its original power of fluorescence. Not only so, but when heated it now possesses the power of spontaneous fluorescence. The same is true for such minerals as fluorspar.

Owing to the presence of the $\gamma$-rays in the Becquerel rays, the latter have the power of ionizing gases, just as the X-rays do. We need not enter into details with regard to this property ; it is obvious that the electroscopic method of determining activity depends upon it.

In many cases the action of the Becquerel rays upon ordinary chemical elements or compounds is well marked. For instance, the Curies have shown that by their influence we can convert oxygen into ozone. ${ }^{1}$ Again, if we submit yellow phosphorus to the action of the radiations, it becomes changed into the red allotropic modifications. ${ }^{2} \quad$ On the other hand, it has been shown by Sudborough ${ }^{3}$ that the presence of radium salts has no influence upon some geometrically isomeric substances which are transmuted into the stable form by the action of light. If we allow the Becquerel rays to act upon a solution of iodoform in chloroform, we shall find that it becomes purple owing to the separation of iodine. ${ }^{4}$ Ammonia ${ }^{5}$ is decomposed by the Becquerel rays; and water ${ }^{6}$ is broken up, yielding oxygen and hydrogen. If a mixture of $d r y$ oxygen and hydrogen ${ }^{7}$ be allowed to remain in contact with radium bromide, however, no combination results. Hydrogen sulphide and sulphur dioxide are both ${ }^{7}$ decomposed by the rays. When air is left in contact with a radium salt over mercury, nitrous

1 Compt. rend., 1899, 129, 823.

2 Becquerel, Compt. rend., 1901, 138, 709.

${ }^{3}$ Sudborough, Proc., 1904, 20, 166.

4 Hardy and Willcock, Proc. Roy. Soc., 1903, 72, 200.

3 Perman, Trans., 1911, 99, 132.

' Usher, Jahrb. Radioaktiv. Elektronik, 1911, 8, 323.

$\checkmark$ Baker, Royal Institution Lecture, March 11, 1911. 
oxide is produced in comparatively large amounts. ${ }^{1}$ The action of the Becquerel rays upon hydrogen peroxide has been studied; ${ }^{2}$ but in this case it is difficult to know exactly how much must be ascribed to the direct action of the rays and how much to the influence of alterations upon the glass of the containing vessel, which, in turn, will affect the rate of decomposition of the peroxide. Other examples ${ }^{3}$ of the chemical effects of the Becquerel rays have been observed, but it is not necessary to deal with them here.

The physiological effects of the radium radiations are very striking. If a radium salt be allowed to remain in contact with the skin for even a few minutes, it is apt to produce extremely painful eruptions; and these are the more troublesome owing to the fact that they take a considerable time to make their appearance. Thus even by bringing radium near the skin, without actual contact, we may produce very deep-seated changes in the tissues without having any outward sign that we have injured them. The action of the radiations upon the tissues is apparently due to the break-down of lecithine, which makes up a considerable part of the epidermis. This destructive character of the radium salts, however, is not without its uses, for in cases of rodent ulcer it has been successfully employed to cure the disease; and at the present time we seem to be on the verge of considerable advances in this direction. Hardy ${ }^{4}$ has found that the coagulation of globulin may be brought about by the influence of the Becquerel rays, and in this case the action appears to be due to the positive charge carried by the $a$-particles.

1 Soddy, Annual Reports, 1911, VIII., 299. 1019.

2 Körösy, Pfluger's Archiv., 1910, 137, 123; Kailan, Monatsh., 1911, 32 ,

3 Lind, Monatsh, 1912, 33, 295; Kailan, ibid., 71; Sitzungsber., K. Akud. Wiss. Wien., 1912, 121, 1329.

4 Hardy, Proc. Physiol. Soc., 1908, XXIX. 


\section{CHAPTER XIV}

THE DISINTEGRATION THEORY AND THE RADIOACTIVE SERIES

\section{The Disintegration Theory.}

AT the time of their discovery, the radioactive properties of the radio-elements seemed capable of explanation on either of two lines. According to purely physical ideas, radium might be regarded as a kind of transformer which took up energy from its surroundings and subsequently emitted the same energy in other forms, just as a fluorescent substance absorbs certain wave-lengths of light and emits the energy thus acquired in the form of a characteristic fluorescence. This hypothesis has now been shown to be invalid. The second view assumes that radium is an unstable material which undergoes decomposition; and that this decomposition is accompanied by the emission of matter and energy. ${ }^{1}$

The disintegration theory, as it is termed, can be very briefly explained. It assumes that the radio-elements are unstable and spontaneously undergo change. During the change, Becquerel rays are emitted and the original radioactive atom is converted into a new atom with a nature different from the parent. This new atom may in its turn be radioactive and emit rays, changing in the process into a third atom, different from its two predecessors. Thus radium emits an $a$-ray and changes to the inert gas niton; niton in its turn emits a new $a$-ray and becomes converted into radium-A; the expulsion of yet another $a$-ray from radium-A leads to the production of radium-B; and after this a $\beta$-ray is emitted and the radium- $\mathrm{B}$ changes into radium- $\mathrm{C}$, and so on.

1 Rutherford and Soddy, Phil. Mag., 1903, 5, 576. 
Thus the ejection of an $a$ - or of a $\beta$-ray from a radioactive element leads to the disintegration of the original element and the formation of a new element which may or may not have radioactive properties.

This theory can be established by evidence of the following nature. Suppose that we have a radioactive element $A$, which disintegrates into a second element $B$, by radioactive change. In any ordinary specimen, we shall have a mixture of $A$ and $B$, since $A$ is always producing $B$. Now let us suppose that A and $\mathrm{B}$ can be separated by chemical means. If we remove all traces of $B$, then we shall have a pure specimen of $A$; but after this has stood for a time it will become contaminated by traces of $B$, which will be formed by the disintegration of $A$. It was an actual experiment of this type which led to the enunciation of the disintegration theory.

Crookes, ${ }^{1}$ in the course of an investigation of the properties of uranium, found that if a uranium salt was precipated by means of ammonium carbonate and the precipitate treated with excess of the reagent, the precipitate almost entirely redissolved, leaving behind it only a very slight residue. The redissolved uranium was found to be radioactively inert, while all the original radioactivity appeared to be concentrated in the small residue. The residue Crookes termed uranium $X$. So far, there was nothing out of the common; it appeared that the activity of uranium was really due to the presence in it of this trace of uranium X. A much more important result was obtained later, however, when it was found that the inactive uranium, after standing for some months, became once more radioactive.*

About the same time, Becquerel ${ }^{2}$ separated uranium and uranium $\mathrm{X}$ in another way. He mixed solutions of uranium and barium salts, and then, on precipitating the barium as sulphate, he found that the barium had acquired radioactive properties, while the uranium had lost them. After standing for a year, the two products were re-examined, and it was found

1 Crookes, Proc. Roy. Soc., 1900, 66, 409.

2 Becquerel, Compt. rend., 1900, 131, 137 ; 1901, 133, 977.

- The activity determinations were made photographically, and are thus concerned only with the $\beta$-rays; had the $\alpha$-rays been used as a test, the uranium would not have appeared to lose much activity. 
that by that time the barium had lost its activity, while the uranium was again as radioactive as it was as the beginning of the experiments.

The only possible conclusion which can be drawn from these results is that the activity of uranium is due to some substance which is produced spontaneously by uranium.

Rutherford and Soddy ${ }^{1}$ found a similar series of phenomena in the case of thorium, and they proceeded to investigate quantitatively the rate at which thorium-X lost its activity. They found that, starting with inactive thorium and active thorium-X, after four days the thorium had regained half its original activity, while in the same time the thorium-X had lost half its radioactive powers. The rate of decay and recovery can be expressed in both cases by exponential equations-

"For the decay of Th X, $\quad \mathrm{I}_{t}=\mathrm{I}_{0} \times e^{-\lambda t}$

For the recovery of $\mathrm{Th}$ activity, $\mathrm{I}_{t}=\mathrm{I}_{0}\left(1-e^{-\lambda t}\right)$

in which $I_{0}$ represents the initial activity of the thorium or the thorium-X; $\mathrm{I}_{t}$ the activity after a time $t$ has elapsed, and $e$ the base of the Napierian logarithms. The factor $\lambda$, it is found, is the same for both decay and recovery. Similar results were obtained with uranium and uranium-X, except that in this case the period during which the uranium-X lost half its activity was about twenty-two days, instead of four as in the case of thorium.

The most striking peculiarity of this decay and regeneration is the fact that it is totally unaffected by changes of temperature, even a white heat appearing to have no accelerating influence. This differentiates it from ordinary chemical reactions, which are all more or less susceptible to changes of temperature.

There is one other point which we must mention. It has been shown that the degree of activity of any salt is directly proportional to the amount of the radioactive element in the salt, and has no connection with the acidic part of the molecule. The activity of radium bromide is different from that of radium carbonate, and depends purely, in each case, upon the percentage of radium metal in the salt.

${ }^{1}$ Rutherford and Soddy, Trans., 1901, 81, 321, 837. 
From the foregoing evidence, we can draw certain conclusions. In the first place, the fact that the percentage of radioactive element present in a salt is the measure of its activity proves conclusively that radioactivity is a property of the atom, and not of the molecule. Secondly, the fact that radioactive change is independent of temperature proves that we are not dealing with an ordinary molecular decomposition. We must therefore have to do with some new atomic property. Finally, since the $a$-particles which are driven out in the Becquerel rays are material bodies, we are obviously witnessing the breakdown of some material system; and from what has gone before it is obvious that this system cannot be a molecular one. It must therefore be atomic.

Another matter may conveniently be mentioned at this point. Let us assume that we have a radioactive substance $\mathrm{X}$ which is disintegrating and yielding a second element $\mathrm{Y}$; and that this element in its turn is disintegrating into a third one $Z$. If we start with a pure specimen of $X$, we shall soon find it contaminated with a certain amount of $\mathrm{Y}$; and the quantity of $\mathrm{Y}$ present would gradually increase, provided no further change were to take place. Actually, however, $\mathrm{Y}$ in its turn is breaking down; so that obviously a time will come when $\mathrm{X}$ is producing $\mathrm{Y}$ at the same rate as $\mathrm{Y}$ is decomposing. The ratio of the amounts of $\mathrm{X}$ and $\mathrm{Y}$ then present will remain constant after this point has been reached; and $\mathrm{X}$ and $\mathrm{Y}$ are then said to be in radioactive equilibrium.

\section{Multiple Disintegration.}

It will be recalled that the $a$-rays of radioactive elements are made up of positively charged helium atoms, whilst the $\beta$-rays are streams of electrons. If an element emits $a$-rays, its disintegration involves a loss of matter from its atom in the form of helium atoms; and consequently its disintegration product must have an atomic weight four units lower than that of the parent substance. On the other hand, the $\beta$-ray change involves only the displacement of an electrical charge and entails no loss of matter; so that the product will have the same atomic weight as the parént.

From this it follows that if a radioactive element is 
susceptible to disintegration along both lines simultaneously it is possible that it may give rise to two different products, one of which will have the same atomic weight as the parent ( $\beta$-ray change) whilst the second product has an atomic weight four units lower than that of the parent ( $\alpha$-ray change).

Several cases of this type have been observed among the radioactive elements. In such cases it is usually found that one mode of disintegration predominates over the other.

\section{The Radioactive Series.}

From what has already been said it will be clear that among the radio-elements we can trace certain family relationships. A given radio-element disintegrates and gives rise to a product; this in its turn produces a third substance, and so on. All these elements stand in a genetic relation to one another and may be regarded as a "radioactive series."

In the earlier days of radioactive research three of these series were recognized and were named, after their parent substances, the uranium, thorium, and actinium series. It has now been shown that the uranium series includes as one of its branches the actinium series also; so that at the present time we have only two series: the uranium series and the thorium series.

To avoid cumbrousness, however, it will be convenient to treat the material in the older form, indicating merely the point of junction of the uranium and actinium series.

The thorium series is the simplest, and we may therefore begin with it. In the following table ${ }^{1}$ (see opposite page) the main facts are given as to the atomic weights, average life, type of rays emitted by the element, and also the group in the Periodic Table in which its properties enable it to be placed.

At thorium-C multiple disintegration occurs, and it is found that thorium- $\mathrm{C}$ gives rise to two disintegration products. One of these, thorium- $\mathrm{C}^{\prime}$, is the product of a $\beta$-ray change. Its atomic weight is therefore the same as that of its parent, viz. 212. The second disintegration product of thorium-C

1 The constants in these tables are taken from Soddy, Chemistry of the Radio-elements (1914). 
is termed thorium-D. It results from an $a$-ray change in thorium- $\mathrm{C}$ and its atomic weight is therefore 208. The disintegration of thorium-C results in the change of 65 per cent. of it into thorium- $\mathrm{C}^{\prime}$ and 35 per cent. of it into thorium-D.

THE THORIUM SERIES.

\begin{tabular}{|c|c|c|c|c|}
\hline Element. & $\begin{array}{l}\text { Atomic } \\
\text { Weight. }\end{array}$ & $\begin{array}{l}\text { Average } \\
\text { Life. }\end{array}$ & Rays. & Group. \\
\hline Thorium & 232 & $2.6 \times 10^{10}$ years & $\alpha$ & $\operatorname{IV} a$ \\
\hline Mesothorium-1 & 228 & $7 \cdot 9$ years & $\beta^{*}$ & II $a$ \\
\hline Mesothorium-2 & 228 & $8 \cdot 9$ hours & $\beta$ & III $a$ \\
\hline Radiothorium & 228 & $2 \cdot 91$ years & $\alpha$ & IV $a$ \\
\hline Thorium-X . & 224 & $5 \cdot 25$ days & $\alpha$ & $\mathrm{II} a$ \\
\hline Thorium Emanation & 220 & 78 seconds & $\alpha$ & 0 \\
\hline Thorium-A • & 216 & 0.2 seconds & $\alpha$ & $\mathrm{VI} b$ \\
\hline Thorium-B & 212 & 15.4 hours & $\beta$ & IVb \\
\hline Thorium.C & 212 & 87 minutes & $\alpha$ and $\beta$ & $\mathrm{Vb}$ \\
\hline
\end{tabular}

Both thorium $\mathrm{C}^{\prime}$ and thorium-D break down to end-products having the atomic weight 208. The constants for the two substances may be given here.

\begin{tabular}{|c|c|c|c|c|c|}
\hline & & $\begin{array}{l}\text { Atomic } \\
\text { Weight. }\end{array}$ & $\begin{array}{c}\text { Average } \\
\text { Life. }\end{array}$ & Rays. & Group. \\
\hline $\begin{array}{l}\text { Thorium-C' } \\
\text { Thorium-D } \\
\text { End-products . }\end{array}$ & $\dot{.}$ & $\begin{array}{l}212 \\
208 \\
208\end{array}$ & $\begin{array}{c}10^{-11} \text { seconds } \\
4 \cdot 5 \text { minutes }\end{array}$ & $\begin{array}{l}a \\
\beta \\
\end{array}$ & $\begin{array}{l}\text { VI } b \\
\text { III } b \\
\text { IV } b\end{array}$ \\
\hline
\end{tabular}

We must now turn to the uranium series and, leaving out of account for the present the branching in the chain which

* This radiation is so soft that it has not yet been experimentally detected. Its existence. however. can be deduced from Soddy's Law (see Chapter XVIII.). 
introduces the actinium series, deal simply with the successive disintegrations which give rise to the radium group.

THE URANIUM-RADIUM SERIES.

\begin{tabular}{|c|c|c|c|c|c|c|}
\hline \multicolumn{3}{|l|}{ Element. } & $\begin{array}{l}\text { Atomic } \\
\text { Weight. }\end{array}$ & $\begin{array}{l}\text { Average } \\
\text { Life. }\end{array}$ & Rays. & Group. \\
\hline$\underset{\downarrow}{\text { Uranium-1 }}$ & - & - & 238 & $8 \times 10^{9}$ years & $\alpha$ & $\mathrm{VI} a$ \\
\hline$\underset{\downarrow}{\text { Uranium- }}$ & - & . & 234 & 35.5 days & $\beta$ & IV $a$ \\
\hline $\begin{array}{c}\text { Uranium }-\mathrm{X}_{2} \\
\downarrow\end{array}$ & . & . & 234 & $1 \cdot 65$ minutes & $\beta$ & $\mathrm{V} a$ \\
\hline $\begin{array}{c}\text { Uranium-2 } \\
\downarrow\end{array}$ & . & . & 234 & $3 \times 10^{6}$ years (?) & $\alpha$ & $\mathrm{VI} a$ \\
\hline$\underset{\downarrow}{\text { Ionium }}$ & - & - & 230 & $2 \times 10^{5}$ years (?) & $\alpha$ & IV $a$ \\
\hline$\underset{\downarrow}{\operatorname{Radium}}$ & . & . & 226 & 2440 years & $\alpha$ & $\mathrm{II} a$ \\
\hline$\underset{\downarrow}{\text { Niton }}$ & . & - & 222 & 5.55 days & $\alpha$ & $\mathrm{O}$ \\
\hline$\underset{\downarrow}{\operatorname{Radium}-\mathrm{A}}$ & $\cdot$ & • & 218 & $4 \cdot 3$ minutes & $\alpha$ & $\mathrm{VI} b$ \\
\hline$\underset{\downarrow}{\text { Radium-B }}$ & - & • & 214 & 38.5 minutes & $\beta$ & IVb \\
\hline Radium-C & . & . & 214 & $28 \cdot 1$ minutes & $\alpha$ and $\beta$ & $\mathrm{Vb}$ \\
\hline
\end{tabular}

At this point, as in the case of the thorium series, a branched chain begins. Radium-C disintegrates simultaneously into radium- $\mathrm{C}^{\prime}$ and radium- $\mathrm{C}_{2}$. We may take the further disintegration of these in succession.

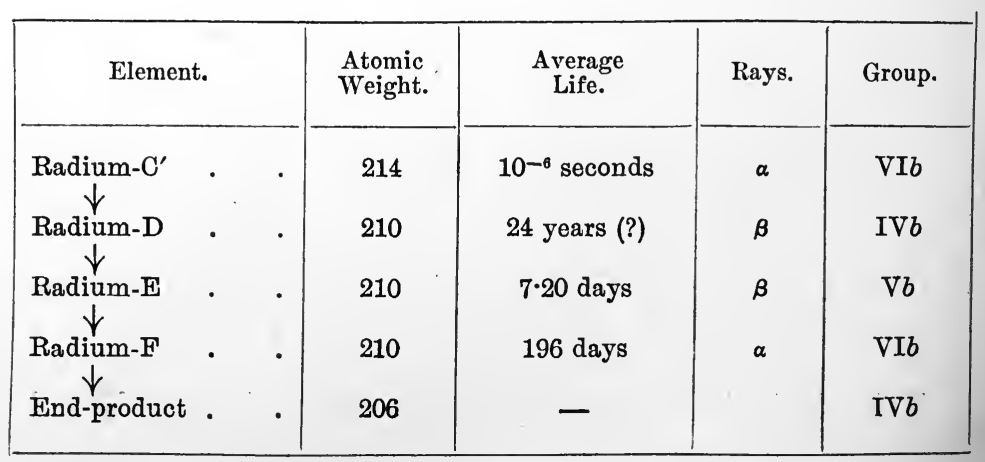


Radium- $\mathrm{C}_{2}$ yields the end-product of its series directly-

\begin{tabular}{|c|c|c|c|c|c|}
\hline . Element. & & $\begin{array}{l}\text { Atomic } \\
\text { Weight. }\end{array}$ & $\begin{array}{c}\text { Average } \\
\text { Life. }\end{array}$ & Rays. & Group. \\
\hline $\begin{array}{c}\text { Radium }-\mathrm{C}_{2} \\
\downarrow \\
\text { End-product }\end{array}$ & . & $\begin{array}{l}210 \\
210\end{array}$ & $\begin{array}{c}1.9 \text { minutes } \\
-\end{array}$ & $\begin{array}{l}\beta \\
-\end{array}$ & $\begin{array}{l}\text { IIIb } \\
\text { IVb }\end{array}$ \\
\hline
\end{tabular}

It is now necessary to deal with the earliest branching in the series of uranium disintegration products and to show the connection between the uranium and actinium series. The matter is one which is not yet ${ }^{*}$ cleared up completely; and further research will probably advance our knowledge of the subject. At present it is only possible to give a provisional account of the problem. ${ }^{1}$

Our present knowledge of the subject can be brought into agreement with either of two alternative schemes for the disintegration of the uranium series-

(I)

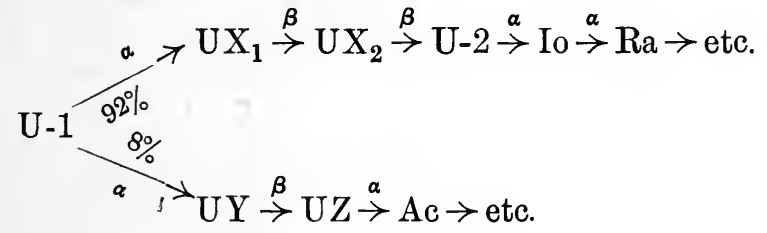

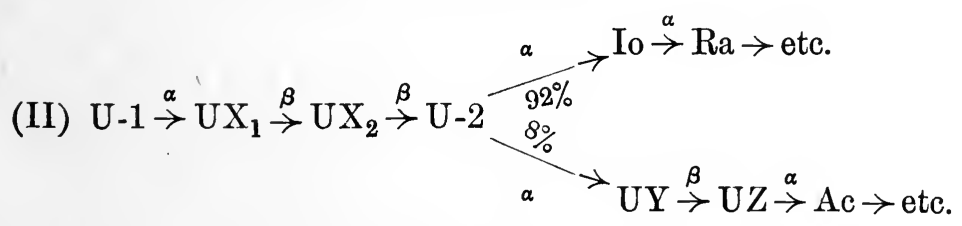

Of these, the former is considered by Soddy to be the more probable. It will be seen that in either scheme the parent of actinium is the element uranium- $Z$, which occupies the position of eka-tantalum in the Periodic Table.

The table on next page shows the constants for the members of the actinium series as far as they have been determined. The atomic weight column is omitted, as definite

* 1920.

' See Soddy and Cranston, Proc. Roy. Soc., 1918, A, 94, 384; compare Hahn and Meitner, Physikal Zeitsch., 1918, 19, 208. 
results have not yet been obtained. Uranium $\mathrm{Y}$ may have an atomic weight of either 230 or 234 , so that doubt is left as to the remainder of the weights.

It will be noticed that in the following scheme no branching of the chain is shown at the bottom, though branching occurs in both the thorium and uranium series as the endproducts are approached. It is possible, however, that there may be a multiple disintegration of actininm-C.

THE ACTINIUM SERIES.

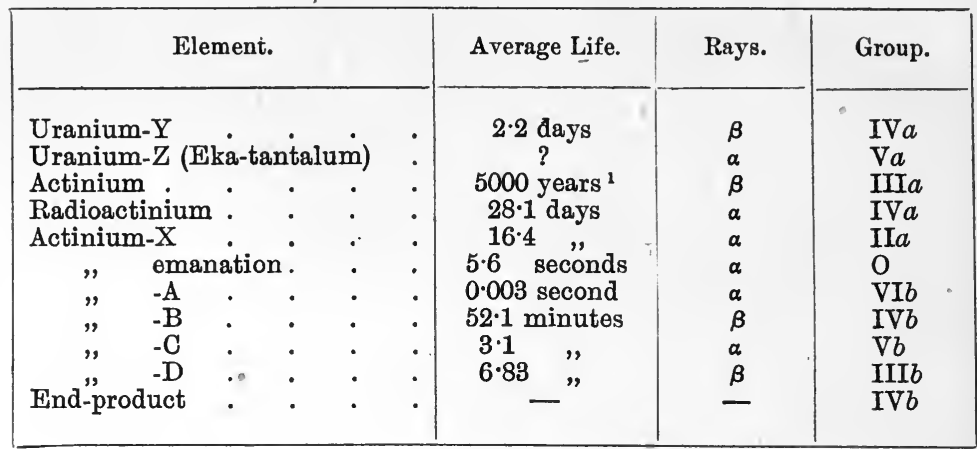

\section{Potassium and Rubidium.}

The numerous investigations ${ }^{2}$ which have been made of the radioactive properties of potassium seem to show that this element possesses distinct activity. Rubidium also appears to have radioactive powers.

\section{Radioactive Recoil.}

We have already seen that, when an a-ray change takes place, a helium atom is ejected by the disintegrating atom. Now since action and reaction are equal, it is clear that the residue of the original atom-the disintegration product-will

1 Soddy and Cranston, Proc. Roy. Soc., 1918, A, 94, 384.

2 McLennan and Kennedy, Phil. Mag., 1908 (vi.), 16, 377; Levin and Ruer, Physikal. Zeitsch., 1908, 9, 248 ; Strong, Physical Review, 1909, 29, 170; Henriot, Compt. rend., 1909, 148, 910 ; Henriot and Vavon, ibid., 149, 30; Büchner, Proc. K. Akad. Wetensch. Amsterdam, 1909, 12, 154; Campbell, Proc. Camb. Phil. Soc., 1909, 15, 11 ; Ebler, Chem. Zeit., 1908, 32, 812. 
move in a direction opposite to that taken by the helium atom, as a rifle recoils when the bullet is fired from it. And just as in the case of the rifle, the momentum of the helium atom and the momentum of the disintegration product will be equal to one another.

It may be well to choose a concrete case so that the matter may be readily grasped. When radium-A disintegrates, it gives rise to a helium atom with atomic weight 4 and an atom of radium-B with an atomic weight 214 . The helium atom is ejected with a velocity of $1.77 \times 10^{9} \mathrm{~cm}$. per second, so that its momentum is $4 \times 1.77 \times 10^{9}$ in atomic units. The momentum of the atom of radium-B is clearly $214 \times v$, where $v$ is the velocity of that atom. Whence we have, since the two momenta are equal in numerical value-

$$
v=\frac{4 \times 1.77 \times 10^{9}}{214}=3.3 \times 10^{7} \mathrm{~cm} \text {. per sec. }
$$

The atom of radium-B formed during the disintegration will thus be moving at a velocity of $3.3 \times 10^{7} \mathrm{~cm}$. per second; and this velocity may be quite sufficient to carry it away from the mass of radium-A in which it was formed. This phenomenon is termed radioactive recoil; and in some cases it forms a means of separating a disintegration product from its parent element, e.g. radium-B and radium-A.

Now if the original radium-A be deposited in a thin layer upon the surface of a plate, it will be evident that, since recoils will be taking place in all directions, half of the freshly-formed radium-B will recoil into contact with the plate whilst the remainder will fly upwards and escape from the plate's surface. In actual practice, some of the particles, even when they are ejected in an upward direction, fail to get clear of the plate. The ratio of the number of atoms of radium-B actually escaping from the plate to the number of atoms which theoretically should escape is termed the efficiency of the recoil.

\section{The Geiger-Nuttall Relation.}

A most interesting relationship has been detected by Geiger and Nuttall ${ }^{1}$ which connects together the period of life of a

1 Geiger and Nuttall, Phil. Mag., 1911, 22, 619; 1912, 23, 439; 24, 647. 
radio-element and the velocity with which it ejects an $a$-particle during disintegration.

The range of a particle is the extreme distance over which that particle can travel through a gas before coming to rest. This range is proportional to the cube root of the initial velocity of the particle. Thus any relation which holds good for the range must also hold good for the velocity of the particle.

Now if the logarithms of the ranges of $a$-particles from a number of different radio-elements be plotted as abscissæ whilst the logarithms of the life periods of the same elements are plotted as ordinates, it is found that a series of straight lines is obtained. Each of the three disintegration series: radium, thorium, and actinium, is found to lie on one straight line; and the three lines lie parallel and quite close together.

It will be seen that this relationship, though entirely empirical, gives us a means of calculating the life period of any radio-element provided that we can measure the range of the $\alpha$-particles emitted by it. Thus it is quite possible to calculate the life period of elements whose existence is so transitory that direct measurement would be difficult.

As to the physical meaning of the Geiger-Nuttall relation, we can suggest nothing definite ${ }^{1}$ but it tends to show that some common link unites all the members of a given series and differentiates them from elements belonging to a different series. As Soddy puts it: ${ }^{2}$ "The atoms in their successive disintegrations preserve a feature distinctive of their origin." But now that the radium and actinium series have been traced to their common origin in uranium, the Geiger-Nuttall relation appears, if anything, more mysterious than ever.

1 See Chapter XIX. for a suggestion as to its meaning and compare Lindemann, Phil. Mag., 1915, 30, 560. Cf. Wolff, Physikal. Zeitsch., 1920, 21, 175.

${ }^{2}$ Soddy, Chemistry of the Radio-elements, Part I., p. 29 (1914). 


\section{CHAPTER XV}

\section{RADIOACTIVE EMANATIONS}

\section{The Emanations of Thorium and Actinium.}

IN a volume of this size, no attempt can be made to deal in detail with the properties of the thirty and more radio-elements; and for a full account of these reference must be made to Soddy's Chemistry of the Radio-elements. Niton, however, has played such a distinctive part in the history of the subject that it deserves description in some detail; and the present chapter will therefore be devoted to an account of the three radioactive gases: thorium emanation, actinium emanation, and niton.

In 1899 Owens ${ }^{1}$ was engaged in an examination of the radiation effects of various thorium derivatives, and in the course of the work he observed that the radiations were by no means constant when measured electrically. Further investigation showed that the inconstancy was due to air-currents about the apparatus; for when the experiments were carried out in closed vessels, the ionization of the air reached a maximum and then remained constant. Now, if radioactive effects can be influenced simply by passing air across the radioactive substance which forms the subject of the experiment, it seems probable that part, at least, of the radioactive influence is due to some material which can be mechanically blown away by the current of air. This material might be either a gas or a cloud of tiny particles of thorium, which had been loosened from the main body of the radioactive mass.

Rutherford ${ }^{2}$ pursued this line of research, and was able to

1 Owens, Phil. Mag. (6), 1899, 48, 360.

2 Rutherford, Phil. Mag. (6), 1900, 49, 1.

I77 
prove that thorium did actually liberate something which we may term an emanation, and that this emanation had radioactive properties. He showed that the properties of the emanation very closely approximated to those of a gas. For instance, it can be blown about by air-currents; it can be bubbled through liquids; a mica stopper will prevent it escaping; and, finally, it causes no deposition of water-globules in the dust-counter. This last experiment proves that the emanation is not a cloud of thorium particles, for these would of course form nuclei for the condensation of water; while the molecules of a gas are too small to produce this effect.

The activity of the emanation was found to decay very rapidly. To measure this decrease in activity, a very ingenious method was employed, the outline of which is as follows. Pure air was blown across some thorium salt, and in this way became mixed with the emanation from the thorium; the mixed gases were then passed into a long brass cylinder through whose walls projected three insulated electrodes. The brass cylinder was insulated and connected with a battery; and the current through the gas was measured by means of an electrometer in the usual way. It was found that the current diminished progressively along the cylinder; and by passing the stream of mixed gases at different rates through the tube, it was possible to determine the rate of decay of the emanation. Le Rossignol and Gimingham ${ }^{1}$ found that the activity of the emanation fell to half value in fifty-one seconds. Thorium emanation condenses at $-120^{\circ} \mathrm{C}$., and the process, which is gradual, is complete at $-155^{\circ} \mathrm{C}$.

In the light of this work, it seemed probable that the other radioactive elements also might give off analogous emanations. Debierne ${ }^{2}$ showed that this was true in the case of actinium, which gives off a gas having properties similar to those of thorium emanation, though its time of decay is very much shorter-about four seconds. It condenses between $-100^{\circ} \mathrm{C}$, and $-143^{\circ} \mathrm{C}$. This substance does not call for detailed treatment, as the general properties of thorium and actinium emanations resemble those of radium emanation, with which we shall now deal in some detail in the next section.

1 Le Rossignol and Gimingham, Phil. Mag. (6), 1904, 8, 107.

2 Debierne, Compt. rend., 1903, 136, 146. 


\section{Niton.*}

The discovery of thorium emanation speedily led to that of the emanation from radium, which was detected in 1900 by Dorn. ${ }^{1}$ It resembles the thorium derivative in most respects, but is much more durable.

When we consider the amount of emanation which is evolved from a given quantity of radium, the first thing which forces itself upon our notice is the difficulty which would be experienced in handling the minute quantity of gas which can be obtained. It has been found that the amount of emanation from 1 gramme of radium in radioactive equilibrium has a volume of about one-tenth of a cubic millimetre. ${ }^{2}$ Now, the usual quantity of a radium salt used by experimenters varies from 20 to 60 milligrammes, and it must be remembered that only a part of this salt is radium. From these figures it will be seen that the volume of emanation obtainable at any time will be very minute. Consequently, it is necessary to devise some method by means of which we can transfer these tiny bubbles of gas from one vessel to another. It has been found that this is best accomplished by mixing the emanation with a large quantity of neutral gas. The mixed gases can then be transferred from vessel to vessel without any appreciable loss of emanation. One of the simplest ways of obtaining the emanation is to pump off the gas from a solution of radium bromide in water. Pure niton can then be obtained by exploding the mixture of hydrogen and oxygen thus produced along with the niton, condensing the niton by cooling with liquid air and pumping off any excess hydrogen and residual helium.

Niton behaves as an ordinary gas. It obeys Boyle's Law, ${ }^{3}$ and diffuses like other gases when placed in a vessel. Experiments have been made by various workers, ${ }^{4}$ with a view to

* This name has been proposed by Ramsay and Gray as a convenient substitute for "radium emanation."

1 Dorn, Abh. d. Naturforscher Gesellsch. Halle, 1900.

2 Gray and Ramsay, Trans., 1909, 95, 1073.

3 Ramsay, Compt. rend., 1904, 138, 1388.

+ Rutherford and Brooks, Trans. Roy. Soc. Can. (2), 1902, 7, 21 ; Chem. News, 1902, 85, 196 ; Curie and Danne, Compt. rend., 1903, 136, 1314 ; Bumstead and Wheeler, Amer. J. Sci. (4), 1904, 17, 97 ; Makower, Phil. Mag. (6), $1905,9,56$. 
determine the molecular weight of niton from its rate of diffusion, but the results are, of course, extremely inaccurate. It must be borne in mind that in these diffusion experiments we are dealing with a very minute quantity of niton mixed with a very large volume of some indifferent gas, so that the results are affected by many factors which do not come into view in ordinary diffusion experiments. All that we can safely conclude from these investigations is that the molecular weight of niton, to judge from its density, must be very high, probably over a hundred. As we shall see later, this estimate is under the mark.

Niton has been liquefied and its critical constants have been determined by Ramsay and Gray. ${ }^{1}$ The liquid emanation is colourless and transparent by transmitted light. It is phosphorescent and shines with a colour which varies with the nature of the glass of the tube in which it is enclosed, the usual tints being green to lilac. When highly compressed, the tint resembles that of a cyanogen flame, being slightly bluish-pink. The solid emanation is not transparent. It melts at $-71^{\circ} \mathrm{C}$. Like the liquid, the solid phase of the emanation is phosphorescent; but the colour of the phosphorescence is much more brilliant, and raries with the temperature. On cooling below the melting-point, the tint of the emitted light is steel-blue; further cooling changes it to yellow, and it finally becomes orange-red. On warming the tube containing it, the colours reappear in inverted sequence. The red phosphorescence disappears at $-118^{\circ} \mathrm{C}$, while at $-59^{\circ} \mathrm{C}$. or - $60^{\circ} \mathrm{C}$. the liquid is dull bluish-green. The critical temperature is $377.5^{\circ}$ absolute, and the vapour-pressure at this temperature is $47,450 \mathrm{~mm}$.

The atomic weight of niton was determined by Gray and Ramsay, ${ }^{2}$ whose method consisted in weighing a given volume of the gas. When it is remembered that the total volume which they were able to obtain at any one time was less than 0.1 c.mm., some idea of the difficulties of the research will be obtained.

Evidence with which we shall deal later in this chapter proves conclusively that niton belongs to the inactive gas

1 Gray and Ramsay, Trans., 1909, 95, 1073.

2 Gray and Ramsay, Proc. Roy. Soc., 1911, A, 84, 536. 
group of elements; and making the usual assumptions with regard to the periodic arrangement of the elements, we can predict that niton will lie somewhere above xenon in the series. Its atomic weight might therefore be either 176 or 222 approximately, as the following figures show, since the difference between two successive atomic weights in the lower section of this group is generally about 45 :-

$\begin{array}{ccccccc}\text { Helium. } & \text { Neon. } & \text { Argon. } & \text { Krypton. } & \text { Xenon. } & \text { I. } & \text { II. } \\ 4 & 20 & 40 & 83 & 130 & 176 & 222\end{array}$

If the figure 222 were correct, then 0.1 c.mm. of niton would weigh less than $1 / 1400$ milligramme; and in order to weigh this small mass with sufficient accuracy it was necessary to devise a balance which would turn with a load of not more than a hundred-thousandth of a milligramme. The production of this balance is certainly the high-water mark of modern physico-chemical technique. ${ }^{1}$ We cannot enter into details of the construction of this apparatus, but must confine ourselves to a brief description of its essentials. The beam of the balance is formed from threads of quartz fibre, and carries a small mirror of platinized silica. Instead of weights, a counterpoise is used which consists of a small quantity of air sealed up in a quartz bulb. The whole balance is surrounded by an air-tight case in which the pressure can be varied by means of a pump. When the air-pressure in the balance is the same as that in the bulb, the apparent weight of the bulb's contents is nil. In a vacuum, the sealed-up air exerts its full weight, as it is not counterpoised by the buoyancy of the air in the case. At any pressure intermediate between ordinary pressure and a vacuum, the apparent weight of the air in the bulb can be calculated. In this way, by varying the pressure of air in the case, we can bring the balance into equipoise; and this can be determined by throwing a beam of light from the platinized mirror on to a scale some feet away from the apparatus. Many corrections are necessary in the course of a weighing, but for an account of these the reader is referred to the original paper. The results obtained by. Ramsay and Gray give a mean atomic weight of niton equal to 223 ; so that niton should lie two places above xenon in the Periodic Table.

1 Steele and Grant, Proc. Roy. Soc., 1919, A, 82, 580. 
The spectrum of niton has been examined by Ramsay and Collie, ${ }^{1}$ who have found that it closely resembles in general characteristics the spectra of the inactive gases. The spectrum fades very soon, and is replaced by the hydrogen spectrum. There is one bright line at 5595; Ramsay and Collie suggest that this may be identical with the line in the spectrum of lightning ${ }^{2}$ which does not seem to have been identified with that given by any known gas.

Like radium itself, radium emanation spontaneously gives out a very considerable quantity of heat. The maximum value of heat liberated per hour from the emanation generated by one gramme of radium is given by Rutherford ${ }^{3}$ as 75 calories; this includes the heat emitted by the disintegration products of the emanation. Niton gives out only a-rays. ${ }^{4}$

It has been shown by Curie and Debierne ${ }^{5}$ that the amount of emanation evolved by radium is independent of the pressure to which the radium is subjected; and it has been found that changes of temperature also appear to be without influence upon the rate of formation.

We must now turn to the chemical nature of niton. It has been shown by Rutherford and Soddy ${ }^{6}$ that the emanations of thorium and of radium both showed an extraordinary inertness even when submitted to the action of strong chemical reagents. For example, no change could be detected in the gas after passing it over red-hot platinum black, or tinely divided palladium, lead chromate, magnesium powder, or zinc dust. Such inertness can be paralleled only by the elements of the argon group; and it seems evident that niton must be reckoned as belonging to that class of bodies. Ramsay and Soddy ${ }^{7}$ have made even more stringent tests, by sparking niton with oxygen in presence of alkali-a process which brings even nitrogen into combination-as well as by passing a mixture of air and niton over a highly heated mixture of magnesium

1 Ramsay and Collie, Proc. Roy. Soc., 1904, 73, 470; Rutherford and Royds, Phil. Mag., 1908, 16, 313; Watson, Proc. Roy. Soc., 1909, A, 83, 50.

2 Pickering, Astrophys. J., 1901, 14, 368.

3 Rutherford, Radioactivity, p. 431 (1913).

- Rutherford and Soddy, Phil. Mag. (6), 1903, 5, 445.

5 Curie and Debierne, Compt. rend., 1901, 133, 931.

6 Rutherford and Soddy, Phil. Mag. (6), 1902, 4, 580 ; 1903, 6, 457.

7 Ramsay and Soddy, Proc. Roy. Soc, 1902, 72, 204. 
powder and lime. In the latter case the mixture of gases was passed for three hours across the magnesium-lime mixture which was heated to a bright redness; the measurements of the radioactivity of the niton made before and after the experiment gave exactly the same result. In the course of these experiments it was found, further, that niton is unattacked even by phosphorus burning in oxygen.

From the results quoted in the foregoing paragraphs, we can now assign to niton a place in the Periodic Table. From the chemical evidence, it is clear that niton is one of the inactive gases; and the evidence of its physical constants makes it practically certain that it must lie below xenon in the Table.

If niton per se is inert, it displays a very great influence when brought into contact with other substances. This influence has nothing whatever to do with the chemical reactivity of the gas, but is due purely to its radioactive powers. We must now mention one or two experiments which have been carried out in this field.

Giesel ${ }^{1}$ noticed that when a solution of radium bromide was allowed to stand, it evolved some gas which investigation showed to be chiefly hydrogen. Ramsay and Soddy ${ }^{2}$ found that the gas mixture contained 29 per cent. of oxygen, the rest being hydrogen. The slight excess of hydrogen they ascribed to contact between the gas mixture and the grease of a tap, which would remove some of the oxygen.

Ramsay $^{3}$ carried out a further series of experiments upon the action of niton on water, and found that there was a mean excess of 5.51 per cent. of hydrogen over and above the quantity required to form water with the oxygen liberated. When the gases were stored over mercury, the percentage of hydrogen was still greater, owing to some of the oxygen being used up in oxidation of the mercury. When mercury is not present, various causes of the presence of the excess of hydrogen might be suggested. It was shown that the corresponding amount of oxygen was not lost by oxidation of the radium bromide to

1 Giesel, Ber., 1902, 35, 3605.

2 Ramsay and Soddy, Proc. Roy. Soc., 1903, 72, 294.

3 Ramsay, Trans., 1907, 91, 931; Cameron and Ramsay, ibid., 1908, 93, 966, 992. 
bromate, nor was there any formation of ozone or hydrogen peroxide; no bromine is liberated from the radium bromide. In all cases care was taken to prevent the gases coming in contact with tap-grease. Ramsay showed, further, that the action of niton is a reversible one; for while, on the one hand; it decomposes water to produce electrolytic gas, it also has the faculty of recombining oxygen and hydrogen to form water again. In later experiments it was found that traces of hydrogen peroxide may be formed by the action of niton upon water.

Enough has been said to show that niton possesses simultaneously two sets of properties which, before its discovery, it would have been hard to believe capable of co-existence. On the one hand, it is itself chemically inert; but, on the other hand, it can influence the chemical properties of other substances to a very marked extent. There is one other property which it exhibits, and this is perhaps the most extraordinary of all. Spontaneously, it disintegrates and yields another element, helium.

Before dealing with the disintegration of niton, however, it will be well to mention some experiments which throw some light upon the earlier stages of the decompositions which it undergoes. Ramsay and Soddy ${ }^{1}$ made an investigation of the volume of the emanation, with a view to determining whether or not the substance remained constant. The results which they obtained are given in the following table:-

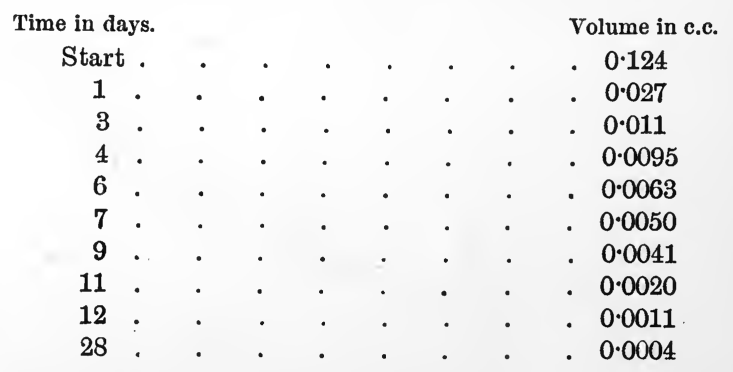

The first number seems very large in comparison with the others; this may possibly be due to an uncondensable gas being present and forced into the walls of the tube.

I Ramsay and Soddy, Zeitsch. physikal. Chem., 1904, 48, 691. 
From these results it is clear that the emanation is gradually disappearing. Now, we need not suppose that matter is being destroyed, but rather that something akin to the condensation of a gas to the liquid state is taking place, which will, of course, be accompanied by a contraction in volume. The simplest hypothesis is that the gaseous emanation which was derived from solid radium is undergoing a further change which is reconverting it into a solid substance.

Here we touch another line of evidence tending to prove the same point, and we must turn aside to consider the phenomena which are classed under the heading excited activity.

It was shown by $M$. and Mme. Curie ${ }^{1}$ in the case of radium, and by Rutherford independently ${ }^{2}$ in the case of thorium, that these substances have the faculty, when placed near other bodies, of communicating to the latter the power of exhibiting the phenomena of radioactivity. Debierne ${ }^{3}$ later found that actinium had a similar property.

We need not describe the experiments which have established the laws governing this phenomenon, but we may summarize the results which have been obtained. It is found that the strength of the excited activity depends, not upon the nature of the object upon which it is located, but purely upon the strength of the activity of the exciting radioactive preparation, and the length of time that it was left in the neighbourhood of the excited object. After the radioactive substance has been removed from the neighbourhood of the excited object, the latter begins to lose its radioactive properties, and the decay of these follows an exponential curve: Further research proved that the excited activity is proportional to the amount of emanation present.

Now, Rutherford ${ }^{4}$ has shown that if a platinum wire is exposed to thorium emanation it becomes endowed with excited activity. If we immerse a wire so treated in hot water, very little change can be detected in the activity when it is withdrawn and dried; but if we immerse the wire in concentrated hydrochloric acid, we shall find that the activity is lost by the

1 M. and Mme. Curie, Compt. rend., 1899, 129, 714.

2 Rutherford, Radioactivity, p. 295.

3 Debierne, Compt. rend., 1904, 138, 411.

- Rutherford, Phil. Mag. (6), 1900, 49, 188. 
wire, but is acquired by the solution; and, further, if we evaporate the solution to dryness, we shall find that the activity has been transferred to the dish. This active matter can be removed from the wire or the dish by simple scraping.

All this goes to show that we are dealing now with a solid substance, and not with a gas. But if we have a solid substance, it might be supposed to be a compound of the emanation with the platinum of the wire. This view is quite untenable, when we consider that red-hot platinum black will not attack the emanation; so we are driven to conclude that the emanation has deposited the solid substance upon the platinum.

Thus the results of measurements of diminution in the volume of the emanation, as well as those phenomena which we have just described, point alike to the view that the emanations of radium and thorium are continually being transformed into solid substances.

Further investigation of the rate of decay in the case of excited activity showed that this solid active deposit (as Rutherford terms it) was not a permanent substance, but was one which rapidly passed through a series of changes. Instead of the decay being expressible by the usual exponential equation-

$$
\mathrm{I}_{t}=\mathrm{I}_{0} \times e^{-\lambda t}
$$

it is found that it really follows the exponential law, but that the total decay series is made up of different factors. The first decay period has one value for $\lambda$, the second has another value for $\lambda$, and the third yet another. This proves, of course, that the primary radioactive deposit is first converted into a second one, having a different radioactive capacity; and that this second one is in turn changed into a third substance which has a decay constant different from that of its predecessors.

In this way the existence of the radium derivatives $A, B, C$, $\mathrm{D}, \mathrm{E}$, and $\mathrm{F}$ has been established, and it has been possible to compare them with other bodies by a comparison of the decay constants of the two substances. The chief point of interest in these substances lies in the fact that radium $\mathrm{D}, \mathrm{E}$, and $\mathrm{F}$ are found in radio-lead, which appears to owe its activity to their presence; while polonium appears to have constants agreeing with those of radium $\mathrm{F}$. 


\section{The Production of Helium.}

Though the measurements of radioactive decay mentioned in the last section were sufficient to show that the emanation from radium was capable of undergoing transformation into other forms of matter, the results were not quite convincing, for, to some extent, the proof depended upon certain theoretical assumptions which were incapable of rigid demonstration. The quantities of the transformation products were extremely small ; and no chemical or spectroscopic experiments could be made which threw much light upon the differences between the various substances. It was not until Ramsay and Soddy ${ }^{1}$ began their work upon the emanation that a product was actually observed whose chemical individuality was beyond doubt.

Rutherford and Soddy, ${ }^{2}$ after finding that niton was an inert gas, put forward the view that it belonged to the argon family; and they further pointed out the fact that helium is always found in minerals which contain uranium or thorium. The question as to whether this association of helium with the radioactive minerals had any connection with their activity was thus opened.

Ramsay and Soddy took $20 \mathrm{mg}$. of radium bromide which had been prepared three months previous to their experiments, dissolved it in water, and collected the gas which was evolved. This gas was for the most part electrolytic gas, which had been produced by the action of the radium and radium emanation upon the water; but it also contained some emanation. To separate the latter from the other gases, the mixture was passed over a red-hot, partly oxidized copper spiral, and the water so formed was removed by means of phosphorus pentoxide. After this the gas was passed into a tiny vacuum tube, in which the spectrum was examined and carbon dioxide detected. This gas was eliminated by means of liquid air, and a re-examination of the spectrum in the small vacuum tube showed the presence of helium, the $\mathrm{D}_{3}$ line being visible. Further experiments were made, and practically all the lines

1 Ramsay and Soddy, Proc. Roy. Soc., 1903, 72, 206; 1904, 73, 346.

2 Rutherford and Soddy, Phil. Mag. (6), 1902, 4, 581. 
in the helium spectrum were found. This work has been confirmed by several workers, ${ }^{1}$ and it has been shown that actinium also gives rise to helium. ${ }^{2}$

In this way, it was proved conclusively that radium emanation actually gives rise to helium. The objection might be made that the helium is present throughout the course of the experiments; but this is shown to be untenable by the fact that the helium spectrum is not visible at first. When an examination is made at the beginning of the process, it is found that a new spectrum is visible which does not contain the helium lines: the latter develop slowly and are visible only some days after the emanation has been brought into the vacuum tube. For instance, in one experiment the emanation was led into the vacuum tube on July 17 ; the new spectrumprobably that of the emanation-was then observed, which contained no helium lines. After standing until the 21st, the helium spectrum was observed, and compared with that of a helium vacuum tube.

It is thus shown that the helium is not present in the earlier part of the experiments, nor is it in any way connected with the presence of the radium salt in the solution: it is derived from the emanation alone. In this way the disintegration hypothesis has obtained its strongest support. Previous to the work of Ramsay and Soddy, the evidence in favour of this hypothesis depended, to a great extent, upon postulates which could not be experimentally tested; but by the production of helium from the emanation, the break-down of one radioactive substance into a non-active body was conclusively demonstrated.

These experiments throw light upon another point. It has been found that helium exists in many minerals, as well as in the waters of several mineral springs; and for a considerable time the presence of this very rare gas under such conditions was inexplicable. Ramsay and Soddy's researches have cleared up this question also ; for it has been shown that both minerals and mineral waters which contain helium have also more or less well-marked radioactive properties. They are therefore

1 Dewar and Curie, Compt. rend., 1904, 138, 190; Meyer and Himstedt, Ann. d. Physik., 1904, 15, 184.

${ }^{2}$ Debierne, Compt. rend., 1905, 141, 383; Giesel, Ber., 1907, 40, 3011. 
certain to contain a small proportion of radioactive emanation, and it is doubtless from this source that helium is derived.

\section{The Disintegration Theory and the Age of Minerals.}

If a mass of uranium were placed in a hermetically sealed vessel and allowed to disintegrate under these conditions, it is evident that either the quantity of helium or the amount of lead which collected in the vessel would give a measure of the period during which the uranium has been enclosed. Calculation shows that one gramme of uranium in radioactive equilibrium with all its disintegration products will give rise to about $11 \times 10^{-5}$ cubic millimetres of helium; so that from the ratio of the quantities of helium and uranium present we can readily ascertain how long the process has been going on. A similar calculation will be possible with regard to the uraniumlead ratio.

Now in certain minerals uranium is present; and the helium given off by it and its disintegration products remains occluded in the solid material instead of being lost to the air. By breaking up the mineral, liberating and estimating the helium and comparing its mass with that of the uranium present, we can arrive at a rough estimate of the period during which the uranium has been confined in the rock under these conditions -in other words, since the rock solidified. Strutt ${ }^{1}$ has investigated the matter and has been able to put forward estimates of the duration of the geological periods based on his results. It must be borne in mind that these estimates are minimum ones, since it is most improbable that all the helium generated is retained by the rocks.

The uranium-lead ratio in minerals is open to even graver criticism when it is taken as a guide to geological age. It can be calculated that one gramme of uranium produces one gramme of lead in 7,500 million years; but this is a very rough approximation. Further, the value of the method depends upon the assumption that during that time no lead has been introduced into the mineral from external sources, which can never be certainly established.

1 Strutt, Proc. Roy. Soc., A, 1908, 81, 272 ; 1910, 83, 298 ; 1911, 84, 379. 
It will be seen that both methods can lead only to approximations to the truth; but they are none the less valuable, in that they give us definite evidence on a period which has hitherto been even more vaguely calculated. The following table ${ }^{1}$ gives some of the main results obtained. The figures represent millions of years which have elapsed since the strata were laid down. Under the heading $\mathrm{He} / \mathrm{U}$ are given the results derived from the helium-uranium ratios; while the column headed $\mathrm{Pb} / \mathrm{U}$ shows the figures deduced from the lead-uranium ratio in minerals. The numbers in the case of the helium ratios show the minimum possible age of the rocks; but the real age is probably much greater.

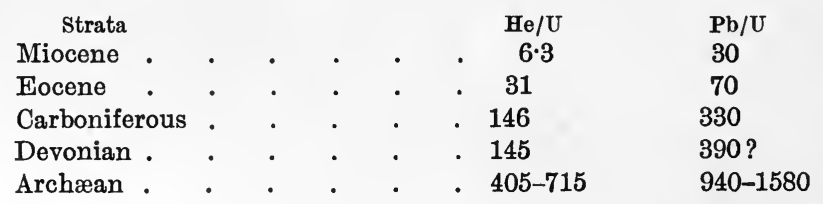

It will be seen that the values differ considerably among themselves, as is only to be expected in view of the difficulties of the problem; but they provide us with a much more accurate gauge than do the previous estimates based on time required for the deposition of the strata.

1 A fuller table is given by Holmes, Discovery, 1920, 1, 108. 


\section{CHAPTER XVI}

\section{ARTIFICIAL TRANSMUTATION}

\section{Introductory.}

THE problem of the transmutation of the elements is as old as the days of the alchemists; and from the modern standpoint it presents a certain interest of its own. Two distinct questions are involved: (1) Is the conversion of one element into another possible? and (2) can this change be accomplished voluntarily in the laboratory with the means at our disposal ?

The progress of radiochemistry has given us the answer to the first question; for the disintegration of the radio-elements has been studied so fully that no doubt can now remain on the point. Certain elements do break up spontaneously and in their disruption they give rise to other materials the elemental nature of which cannot be denied. But since the radioactive processes are entirely beyond our control, the second question still remains unsettled.

From the practical standpoint, the transmutation question is approachable on two sides: the synthetic and the analytic. We may attempt to build up complicated atoms from two or more simpler ones; or we may endeavour to copy the radioactive processes and break up complex atoms into simpler materials. In the following sections, an account will be given of the researches with appear to bear upon the subject; but it must be borne in mind that the question is still an open one.

\section{The Work of Ramsay and Cameron.}

It has already been mentioned that either radium or radium emanation, when brought into contact with water, decomposes 
the latter just as an electric current does. Apparently this action is confined to the liquid state, for steam does not appear to be affected in the same manner. ${ }^{1}$ Hydrochloric acid is broken down into hydrogen and chlorine under the same circumstances. This parallel between the actions of the emanation and the electric current led Ramsay ${ }^{2}$ to try the effect of niton upon a solution of copper sulphate, from which, by analogy, he expected to get copper deposited. When this experiment was carried out, Ramsay detected no helium in his apparatus, but observed the presence of neon and argon. Traces of lithium were found in the copper salt solution after the experiment was completed, though no lithium was found at the end of a blank experiment in which no niton was employed.

Further results were published later. ${ }^{3}$ In every case, lithium was found when niton had been employed, whilst no lithium was detected in exactly similar experiments wherein no niton was brought into contact with the solution of the copper salt.

Again, ${ }^{4}$ when a large quantity of thorium nitrate was left sealed up in a bulb for several months, it was found that a certain amount of carbon dioxide was detected among the gases evolved. The action of niton upon thorium nitrate solution resulted in the detection of carbon dioxide in this case also ; and similar results were observed in the cases of zirconium nitrate and hydrofluosilicic acid.

Ramsay suggested that the action of the niton had broken down the complex atom of copper into lithium, which is the lightest element in the same family; and that in a similar manner the atoms of thorium, zirconium, and silicon had been degraded into carbon.

A repetition ${ }^{5}$ of the lithium-copper experiment by Mme. Curie and Mdlle. Gleditsch led to negative results; and Rutherford and Royds ${ }^{6}$ failed to detect the presence of neon.

1 Cameron and Ramsay, Trans., 1908, 93, 966.

2 Ramsay, Nature, 1907, 76, 269.

3 Cameron and Ramsay, Trans., 1907, 91, 1593 ; 1908, 93, 992.

4 Ramsay, Trans., 1909, 95, 624; Ramsay and Usher, Ber., 1909, 42, 2930.

5 Curie and Gleditsch, Compt. rend., 1908, 147, 345 ; cf. Perman, Trans., 1908, 93, 1775.

6 Rutherford and Royds, Phil. Mag., 1908, 16, 812. 


\section{The Investigations of Collie and Patterson.}

An account of experiments in transmutation would be incomplete without some mention of the following results; but it must be noted that the experimenters themselves have never described their results as due to the transmuting of hydrogen into other elements. They have confined themselves in their papers to a description of their experimental work and have put forward no theory on the subject.

Collie and H. S. Patterson ${ }^{1}$ observed that when pure calcium fluoride was bombarded with cathode rays a mixture of gases was evolved. This mixture consisted chiefly of hydrogen, oxygen, and carbon monoxide; but when sparks were passed through it and the residue was condensed by means of charcoal and liquid air, a small quantity of neon was detected. The same result was obtained when carefully purified glass wool was substituted for the calcium fluoride.

There are obviously only two possible sources of this neon. It must either have leaked into the tube from outside or it must have originated in the interior of the tube.

With regard to the leakage possibility, various tests were tried. Of these, the crucial one was as follows. The discharge tube was sealed inside another glass tube which was filled with neon at about half an atmosphere pressure; hydrogen at low pressure was then admitted into the inner tube, through which the cathode discharge was passed.* After the experiment, the hydrogen was pumped out of the inner tube and on examination it was found admixed with just the same quantity of neon as in previous cases. Had there been any leak in the discharge tube; or had the glass of the discharge tube become permeable to neon under the action of the cathode ray, then a much greater quantity of neon might have been expected to make its way through the intervening wall in the second experiment. In the first experiment the pressure of neon outside the vessel is about $1 / 55,000$ of an atmosphere, whilst in the second experiment with the enclosed tube the neon surrounding the discharge tube was at a pressure more than twenty thousand times as great. If a leak were in

1 Collie and H. S. Patterson, Trans., 1913, 103, 264.

* No calcium fluoride or glass wool was used in this experiment. 
question, the amount of neon found in the tube during the second experiment would be far greater than that observed with the unjacketed tube; and the practical coincidence of the results in the two cases appears to exclude leakage as a possible explanation of the phenomena. Further, if neon had leaked in from the air during the first experiment, a corresponding quantity of argon would also have found its way into the discharge tube; and as there are about 500 parts of argon to 1 part of neon in the atmosphere, the argon spectrum could not possibly have escaped detection had any air penetrated into the cathode tube.

It must therefore be taken as proved that the neon originates within the discharge tube; and the problem of its origin is narrowed down. Only three sources can be suggested: the glass of the tube; the metal of the electrodes; and the hydrogen which the discharge tube contains.

It was proved that when the glass was finely powdered, washed with chromic acid, and heated in an exhausted tube until it fused, no neon was evolved. A similar negative result was obtained when the aluminium electrodes were fused in a vacuum: hydrogen was evolved from the metal but no neon made its appearance. As to the hydrogen, it contained no neon before the experiment.

A further series of experiments led to even more surprising results. The jacketed tube was employed; and in this case the external tube was exhausted. After the experiment, the usual amount of neon was found in the inner tube. When oxygen was admitted into the outer jacket after the experiment and pumped out again it was found that sparking of this oxygen produced a slight explosion, pointing to the presence of hydrogen which must have been driven through the intervening wall between the two tubes by the violence of the cathode discharge. The mixture from the outer jacket was then treated with charcoal and liquid air in the usual way; and it was found that the uncondensable residue contained chiefly helium mixed with a small quantity of neon. When the discharge experiment was repeated with oxygen in the jacket, the usual quantity of neon was detected in the inner tube, at the end of the experiment; but the oxygen was then found to contain neon also, mixed with a little helium. 
Repetitions of the experiment under varying conditions showed that when the outer tube was completely exhausted before the discharge passed in the inner tube, helium predominated over neon in the outer tube after the discharge; whereas when oxygen at pressures up to $15 \mathrm{~mm}$. was present in the outer tube, neon was the main new product of the discharge and helium was subsidiary.

Finally, ${ }^{1}$ Collie and Patterson showed that the presence of electrodes in the tube is not necessary for the production of the phenomena ; for similar results are obtained when a powerful oscillating discharge is passed through a coil of wire wrapped round the outside of an electrodeless tube containing hydrogen at low pressure. Considerable quantities of hydrogen disappeared from the interior of the tube when the current was running. A gas made its appearance in the tube and showed the carbon spectrum when examined. It may possibly be $\mathrm{X}_{3}$ * $^{*}$

Soddy and Mackenzie, ${ }^{2}$ Strutt, ${ }^{3}$ Merton, ${ }^{4}$ and Egerton ${ }^{5}$ were unable to obtain the results of Collie and Patterson; though Masson ${ }^{6}$ and Thomson ${ }^{7}$ confirmed them. Collie, ${ }^{8}$ using Merton's actual apparatus, bombarded uranium powder in an atmosphere of hydrogen, and showed that both helium and neon were produced. The production of the former is perhaps not astonishing; though no helium was observed when the uranium was raised to a red-heat in vacuô for an hour: but the production of the neon cannot be ascribed to any ordinary radioactive decomposition of the uranium.

One factor which appears to exert considerable influence is the nature of the interrupter used on the coil generating the current. With a 10-inch coil and platinum break, positive results were obtained; whilst only negative results were

* See p. 149.

1 Collie and H. S. Patterson, Proc., 1913, 29, 217.

2 Soddy and Mackenzie, Proc. Roy. Soc., 1908, A, 80, 92. (This paper, as can be seen from the date, is antecedent to the work of Collie and Patterson.)

3 Strutt, Proc. Roy. Soc., 1914, A, 89, 499.

4 Merton, Proc. Roy. Soc., 1914, A, 90, 549.

5 Egerton, Proc. Roy. Soc., 1915, A, 91, 180.

- Masson, Proc., 1913, 29, 233.

7 Thomson, Nature, 1913, 90, 645; Rays of Positive Electricity, p. 122 (1913).

${ }^{8}$ Collie, Proc. Roy. Soc., 1914, A, 90, 554. 
observed during the use of a larger coil with a mercury break.

A summary of the evidence is to be found in a paper by Collie, Patterson, and Masson. ${ }^{1}$

\section{Rutherford's Experiments.}

When a-particles from a radioactive source are allowed to pass through an atmosphere of hydrogen it is found that scintillations can be observed on a fluorescent screen which is placed beyond the normal extreme range of the a-particles. This implies that under these conditions something is striking the screen; and since the screen and the radioactive source are separated by a distance which no a-particle can span, it is evident that this "something" cannot be an $a$-particle. The explanation of the phenomenon ${ }^{2}$ appears to be as follows. To take an extreme case, let us imagine that an a-particle meets a hydrogen atom "end-on" and that a collision occurs. Since the hydrogen atom is four times lighter than the $a$-particle, it is evident that it will recoil after the collision at a speed much greater than that possessed by the $a$-particle; and being endowed with this greater velocity it will be able to travel through a longer distance than the original $a$-particle would have done before coming to rest at the end of its normal range. It is therefore assumed that the scintillations on the screen are produced by these recoiling hydrogen atoms, which are termed H-particles.

Rutherford has examined the phenomenon in the case of gases other than hydrogen, and in the case of nitrogen ${ }^{3}$ he obtained an anomalous effect. When a stream of $a$-particles was passed through chemically pure nitrogen, it was found that the range of the recoiling atoms, instead of being small-as might have been expected when the relative masses of the $a$-particle and the nitrogen atom are compared-was comparable to that which would have been produced if hydrogen had been present instead of nitrogen. In fact, the penetrating

1 Collie, Patterson, and Masson, Proc. Roy. Soc., 1914, A, 91, 30.

2 Marsden, Phil. Mag., 1914, 27, 824; Marsden and Lantsberry, ibid., 1915, 30, 240.

${ }^{3}$ Rutherford, Phil. Mag., 1919, 37, 581 . Cf. Hinsberg, Chem. Zeit., 1920, 44, 294. 
power of these new particles was found to be even greater than that of the $\mathrm{H}$-particles from hydrogen.

If these particles should eventually be proved to be $\mathrm{H}$ particles, there seems to be no escape from the idea that the nitrogen atom has been shattered by the impact of the $a$-particle upon it; and that hydrogen is one of the disintegration-products. In support of this view, it is urged that the atomic weight of nitrogen corresponds almost exactly to the united weights of three helium and two hydrogen atoms; so that the atom of nitrogen may be built up from three helium nuclei and two hydrogen nuclei.

If such a disruption of the nitrogen atom takes place under the action of $a$-particles, it might be suggested that traces of hydrogen should be detectable in a vessel filled with a mixture of niton and nitrogen or in the gases evolved from thorium nitrate.

Should the Rutherford particles eventually prove to be hydrogen atoms, it will establish the correctness of Ramsay's view (which was rejected at the time by more than one expert in radioactive problems), that the energy of radioactive bombardment might suffice to disintegrate atoms which normally are stable. 


\section{CHAPTER XVII}

\section{THE ISOTOPES AND SODDY'S LAW}

\section{Soddy's Theory of Isotopic Elements.}

IN 1910 , Soddy ${ }^{1}$ called attention to the fact that the evidence then accumulated proved that certain members of the radioactive group of elements exhibited a complete identity of chemical behaviour. Thus thorium-X, actinium- $X$, and radium were found to be chemically indistinguishable from one another; ionium, radiothorium, radioactinium, and thorium were also endowed with identical properties so far as chemical reactions went; and the three emanations could not be distinguished from each other by any chemical means.

As Soddy indicated, we have here three groups of substances in which each element is readily distinguishable from the rest by means of its radioactive constants; but in which no member of a group can be isolated from its neighbours by the employment of purely chemical methods of separation.

When the atomic weights of these substances are examined, it is found that they are not identical, That of ionium is 230 , of thorium 232 , and of radiothorium 228 . It thus became clear that there could exist two or more elements with different radioactive constants and different atomic weights but with identical chemical properties. Soddy hazarded the conjecture - which has since proved well-founded-that these phenomena would prove to be the beginning of some embracing generalization which would throw light, not only on radioactive processes, but on the elements in general and the Periodic Law.

In 1911, Soddy carried his views to a further stage.

1 Soddy, Chemical Society's Annual Reports, 1910, VII., 285 ; see also Soddy, The Chemistry of the Radio-elements, 1st edition, I., 29 (1911). 
Chemical analysis, as consideration will show, is not necessarily a method of separating matter into homogeneous elements; but is, instead, a mode of isolating from each other certain types of matter which differ in behaviour when treated with a given reagent. Hitherto it has been assumed that, when chemical analysis has done its best, the resulting substances are entirely homogeneous or, as we say, elemental forms of matter. But are we justified in this conclusion? Suppose that we had a mixture of thorium and ionium. No chemical process with which we are acquainted at present will separate these two bodies from one another; yet when we apply a totally new method of analysis-radioactive analysis-we find that we are not dealing with one substance but with a mixture of two.

Chemical analysis, then, permits us to separate matter into a number of "types" which are homogeneous in chemical behaviour. Each "type" is recognizably different from other "types" by means of its chemical reactions; but may or may not be homogeneous as regards other properties such as atomic weight or stability.

To express this idea, Soddy coined the word isotopes. A group of two or more elements occupying the same place in the Periodic Table and being chemically identical and non-separable is defined as a group of isotopes; and within a group the separate members are said to be isotopic with one another. Thus ionium, thorium, and radiothorium are isotopes; the three emanations are isotopic with one another; and mesothorium-1 is an isotope of radium.

This conception of isotopy is so alien to all the old-fashioned views of the elements that it deserves further elaboration here. It is natural to inquire whether isotopes are really inseparable and indistinguishable from one another; and whether, if they cannot be entirely separated, they are not partially separable -i.e. whether a series of mixtures of them in unequal quantities cannot be obtained by the use of particular methods of separation.

The work of Paneth and von Hevesy ${ }^{1}$ on radium-D and lead leads to the conclusion that ordinary modes of separation

1 Paneth and von Hevesy, Sitzungsber. K. Akad. Wiss. Wien, 1913, 122, 993 ; Monatsh., 1913, 34, 1393. 
are quite incapable of producing the desired result. Precipitation, distillation, adsorption, electrolysis of aqueous solutions or of the fused material, diffusion and dialysis all failed to yield any detectable result; and when the delicacy of radioactive measurements is considered, this seems to prove that very little doubt can remain on the point.

The most severe test to which the theory of isotopes could be subjected was applied by Paneth and von Hevesy ${ }^{1}$ in the following manner. In the first place, they showed that the decomposition potential at which radium-E is deposited on the cathode is altered in the same direction and to the same extent by the addition of the isotopic element bismuth as it would be by the addition of the same number of ions of radium-E. Similar results were obtained in the case of thorium-B and its isotope, lead. In the second place, in the deposition of radium- $\mathrm{E}$ and of thorium-B at potentials below the decomposition voltage, the addition of isotopic elements prevents this decomposition though other.elements are without influence. Finally, ${ }^{2}$ when a galvanic chain was formed in which radium-D was one of the members, the potential of the chain was found to be practically the same as that of a similar chain wherein ordinary lead was substituted for the radium-D. The voltage in the former case was -0.884 volts; whilst in the latter it was -0.888 volts.

With regard to the spectra of isotopes, it is too early to decide whether or not they are identical, though the evidence already at our disposal tends to show that no difference can be detected between them. ${ }^{3}$ Thus an ionium-thorium mixture containing at least 10 per cent. of ionium showed not a single line which was not already known in the thorium spectrum. It is regrettable that the life periods of the emanations from thorium and actinium are so short, as they would yield decisive evidence when their spectra were compared with that of niton.

It might at first sight be supposed that the isotopes furnish a case parallel to that of the elements of the rare earths; but this is not so. In the rare earths the difficulty encountered

1 Paneth and von Hevesy, Physikal. Zeitsch., 1914, 15, 797.

2 See also Klemensiewicz, Compt. rend., 1914, 158, 1889.

3 Exner and Haschek, Sitzungsber. K. Akad. Wiss. Wien, 1912, 121, IIa.. 1075 ; Russell and Rossi, Proc. Roy. Soc., 1912, A, 87, 478. 
does not lie in separating one element from another but rather in discovering whether or not a separation has been attained. In the series of isotopes it would be very simple to ascertain the reality of a separation could it be achieved; but up to the present no separation has been carried out.

\section{The a-Ray Change.}

To Soddy also we owe the next step in advance in our knowledge of the rationale of radioactive change, ${ }^{1}$ his publication on the subject dating from 1911.

When a helium atom is expelled by a radioactive element, it carries with-it two positive charges; and the disintegration product is therefore characterized by an electrical charge two units less positive-or more negative-than the original element. At the same time, owing to the loss of four units of matter in the form of the helium atom, the atomic weight of the disintegration product is four less than that of the parent substance.

Now the loss of one positive charge by an atom may be regarded as equivalent to a diminution of one positive valency of that atom; which is the same as a shift of one column to the left in the Periodic Table. The loss of two positive charges would therefore correspond to shifting an element into the second column to the left in the Table.

Taking the two changes together, Soddy enunciated the rule that the expulsion of an $a$-ray by an element results in the formation of a new element which has an atomic weight four units less than that of its parent and which is situated two columns to the left in the Periodic Arrangement.

For example, radium has an atomic weight of 226 and it lies in Group II. of the Table. As a result of the a-ray change it passes into niton which has an atomic weight of 222 and which lies in Group 0-two columns to the left of Group II. Again, niton in its turn ejects an $a$-particle and becomes converted into radium-A which has an atomic weight of 218 and which occupies a position in Group VI.* of the Periodic

1 Soddy, Chemistry of the Radio-elements, Part I., 30 (1911).

* This example has been chosen to illustrate a particular point; but it must be understood that our knowledge of the chemical character of radium-A came at a later date from Fleck's investigations, of which an account is given in the next section. 
Arrangement. This last change may be rather difficult to grasp, but possibly the following reasoning may make it clearer. Niton loses two positive charges (which are equivalent to two positive valencies) during the $a$-ray change. But niton starts by having no valency at all. The loss of two positive valencies is therefore simply equivalent to the gain of two negative valencies; so that the new element is characterized by two negative valencies; and this brings it into the sulphur column of the Table.

\section{Fleck's Investigations of the Chemistry of the Radio- elements.}

The next advance in our knowledge of the subject is due to the work of Fleck ${ }^{\mathbf{1}}$ which was carried out at the suggestion of Soddy. The object of the investigation was to find the common element which each radio-element most resembled and then to see whether, after the two had been mixed, any separation of the constituents was possible by chemical means. At the time when this investigation was begun, very little was known about the chemistry of the "active deposit" group; but Fleck's researches established the relationships between some sixteen radio-elements and also showed their kinship with certain non-radioactive elements.

The difficulties of the research can hardly be over-estimated. In the first place, some of the substances examined could be obtained only in minute traces. Secondly, the average life of the elements was, in some cases, extremely brief. Thirdly, owing to the rapidity with which the substances became contaminated with disintegration products, radioactivity measurements were complicated by subsidiary factors.

It is impossible to give here a detailed account of all the various examinations made by Fleck; but for the present purpose it will be sufficient to describe a single example, which will give some idea of the difficulties which had to be overcome. The case of thorium-B and thorium-C may be chosen.

First as to the manner in which the two products can be identified. Thorium-B emits only $\beta$-rays, whilst Thorium-C yields a-rays. If a complete separation of the two compounds

1 Fleck, Trans., 1913, 103, 391, 1052. 
is effected, then the thorium-B will be inactive at first as far as $a$-ray measurements go but will show a gradually increasing a-radiation owing to the formation of thorium-C by disintegration. On the other hand, thorium-C will show a gradual falling off in $a$-radiation owing to its disintegration.

Fleck found that when a small quantity of a lead salt was added to a solution containing thorium- $\mathrm{B}$ and thorium- $\mathrm{C}$ in radioactive equilibrium, complete precipitation of the lead as sulphide carried down both of the radio-elements with it. Incomplete precipitation led to a preponderance of thorium-C in the sulphide precipitate. When, in addition to the lead, another metal was present which gave a sulphide soluble in ammonium sulphide, no activity was found in the ammonium sulphide solution. This proves that neither thorium-B nor thorium-C is allied to the noble metals.

A mixture of the two radioactive materials with salts of lead and copper was then tested. It was found that when the solution of the mixture was precipitated with sodium carbonate and the precipitate was digested with potassium cyanide solution, no activity was found in the solution. This demonstrates that the radio-elements in question must be akin to either lead or bismuth, since these are the only two metals of the second analytical group which behave in this manner.

A mixture of lead and bismuth salts with salts of the two radio-elements was then treated with sulphuric acid and alcohol. The precipitated sulphate was removed and the bismuth was precipitated by means of sulphuretted hydrogen. Radioactivity measurements proved that the lead sulphate precipitate contained thorium-B whilst the bismuth sulphide preciptate contained the thorium-C sulphide. This established the resemblance between thorium-B and lead on the one hand and between thorium-C and bismuth on the other.

Many other methods were tested in the attempt to separate thorium-B from admixed lead and thorium-C from admixed bismuth; and all led to the same conclusion. Among them may be mentioned the following: (1) Precipitation of lead as sulphate and of bismuth as sulphide; (2) Precipitation of lead as sulphate and of bismuth as hydroxide; (3) Precipitation of lead as chloride; (4) Precipitation of lead as sulphide and of bismuth as oxychloride; (5) Precipitation of lead as lead 
hydroxide and of bismuth by means of $m$-nitrobenzoic acid; (6) Precipitation of bismuth with alcohol; (7) Electrolytic separation; (8) Fractionation of lead by means of sulphuric acid and alcohol.

It will be seen that these reactions prove conclusively that no separation of lead from thorium-B or of bismuth from thorium- $\mathrm{C}$ can be attained by the ordinary methods.

This series of investigations by Fleck established the correctness of Soddy's a-ray rule; and also led on to the further generalization with which we are about to deal.

\section{The $\beta$-Ray Change.}

The credit of throwing light upon the effect of $\beta$-ray changes is shared by no less than four investigators who almost simultaneously and, possibly, independently had arrived at solutions of the problem. ${ }^{1}$. It was pointed out by them that the expulsion of a $\beta$-ray ${ }^{*}$ from a radioactive element resulted in the formation of a disintegration product with a chemical character which placed it in the column to the right of that which is occupied by the parent substance.

For instance, uranium- $\mathrm{X}_{2}$, which belongs to Group V., ejects a $\beta$-ray and is converted into uranium-2, which falls into Group VI.; radium-B, in Group IV., throws off a $\beta$-ray and yields radium. $\mathrm{C}$, which belongs to Group $\mathrm{V}$.

Thus the $\beta$-ray change is different from the $a$-ray change both in nature and in degree. The a-ray change, in the case of an element in Group N, results in the production of a substance belonging to Group $(\mathrm{N}-2)$; whilst the $\beta$-ray change gives rise to a new element which lies in Group $(N+1)$. Not only so, but the a-ray change results in a.loss of four units of atomic weight; whilst the $\beta$-ray change yields a new element with the same atomic weight as that of its parent.

1 von Hevesy, Physikal. Zeitsch., 1913, 14, 49 (January 15); Russell, Chem. News, 1913, 107, 49 (January 31); Soddy, ibid., 97 (February 28); Jahrb. Radioaktiv. Elektronik, 1913, 10, 188; Fajans, Physikal. Zeitsch., 1913, 14, 131, 136 (February 15). Of the four, only Soddy and Fajans produced correct results. See footnote on p. 205.

* "Rayless" changes are probably actually $\beta$-ray changes in which the $\beta$-ray is of a penetrating power so feeble as to defy identification at present. 


\section{Soddy's Law.*}

We are now in a position to fit together into a connected whole the three main facts with regard to the relations between the Periodic Table and the course of the disintegration of the radio-elements.

Soddy's Law may be stated as follows. When a radioelement expels an $a$-particle, the disintegration product takes up a position two places from the parent substance in the direction of diminishing mass: so that the product is not in the next family but in the next but one; and the atomic weight of the product is four units lower than that of the parent substance. In a $\beta$-ray change, the product retains the same atomic weight as the parent, but occupies a position in the Periodic Table in the column next the parent substance and on the side opposite to that on which the product of an $a$-ray change would lie.

It is evident that the successive operations of an $a$-ray change and two $\beta$-ray changes, in any order, will produce a final disintegration product which lies in the same column of the Periodic Table as the original parent did. Thus, for example, in the case of uranium we have an element lying in Group VI. It throws off an $a$-particle, producing uranium- $X_{1}$, which belongs to Group IV. A $\beta$-ray change yields uranium$\mathrm{X}_{2}$, a Group $\mathrm{V}$. element; and a further $\beta$-ray change gives rise to uranium-2 which belongs to Group VI. Thus, starting with an element in Group VI., we have ended with a new element in the same Group though with an atomic weight four units less than that of the original uranium. Again, take the case of actinium-B. This element lies in the lead Group. It ejects

* Hitherto there has been no name to distinguish this law and it seems desirable that there should be some concise mode of referring to it. The main credit in the matter is due to Soddy, who first grasped the meaning of the isotopes and the a-ray change. To him also is due the initiation of Fleck's researches which definitely established the validity of the law. Russell, when enunciating the rule for the $\beta$-ray change, combined it with Soddy's previous $\alpha$-ray rule; but owing to the fact that he failed to regard the Periodic Table as continuous from Group I. through Group 0 to Group VII., his statement of the law was somewhat imperfect. Soddy and Fajans practically simultaneously published the complete generalization; but from the foregoing it will be seen that the lion's share of the credit belongs to the former investigator. Fleck's results, though unpublished, seem to have been known to those interested in the subject at the time. 
a $\beta$-particle and yields actinium-C which belongs to the bismuth Group. Actinium-C now expels an $a$-particle and produces actinium-D, an element in the thallium Group. Finally, actinium-D throws out a $\beta$-particle and passes into the endproduct of the series. This end-product lies in the lead Group from which we started.

An examination of Fig. 26, shown on p. 207, will make clear the effects of the two types of radioactive change. The Table is the original one drawn up by Soddy ${ }^{1}$ in 1913 and therefore does not show * theconnection between the uranium and actinium series which has already been discussed on a previous page. It will be noticed that Soddy's diagram expresses the fact that there is a unit difference of charge between successive places in the Table, thus anticipating Moseley's independent deduction drawn from the X-ray spectra of the elements.

Inspection of the diagram will show that in certain columns two or more elements are to be found. For example, at the right-hand side in Group VIa. we find uranium-1 and uranium-2, whilst in some of the other columns the numbers are even greater, reaching a maximum in Group IVb. The result of Fleck's investigations proved conclusively that elements which occupy the same place in the Periodic Table cannot be separated from one another by ordinary chemical means. This holds good whether these elements belong to the same radioactive series or not. For example, radio-actinium, thorium, and ionium, though each belongs to a different series, are chemically inseparable from one another. They belong, as has already been indicated, to the class named by Soddy the isotopes. If we mix thorium and ionium together we shall not be able to separate them again by any process of ordinary chemical analysis, though by applying radioactivity tests we could determine that more than one element was present.

Soddy ${ }^{2}$ has drawn attention to some of the consequences which follow from the recognition of isotopy. In the first place, the position occupied by an atom in the Periodic Table is not a mere function of its mass but depends mainly upon

1 Soddy, Paper in Section B, of the British Association, 1913.

2 Soddy, Chemistry of the Radio-elements, Part II., 6 (1914).

* Soddy's latest diagram is to be found in his lecture to the Chemical Society (Trans., 1919, 115, 16). 
the electrical content of the atom and is only to a subsidiary extent influenced by atomic mass. From this it follows that chemical elements are not necessarily homogeneous but may be,

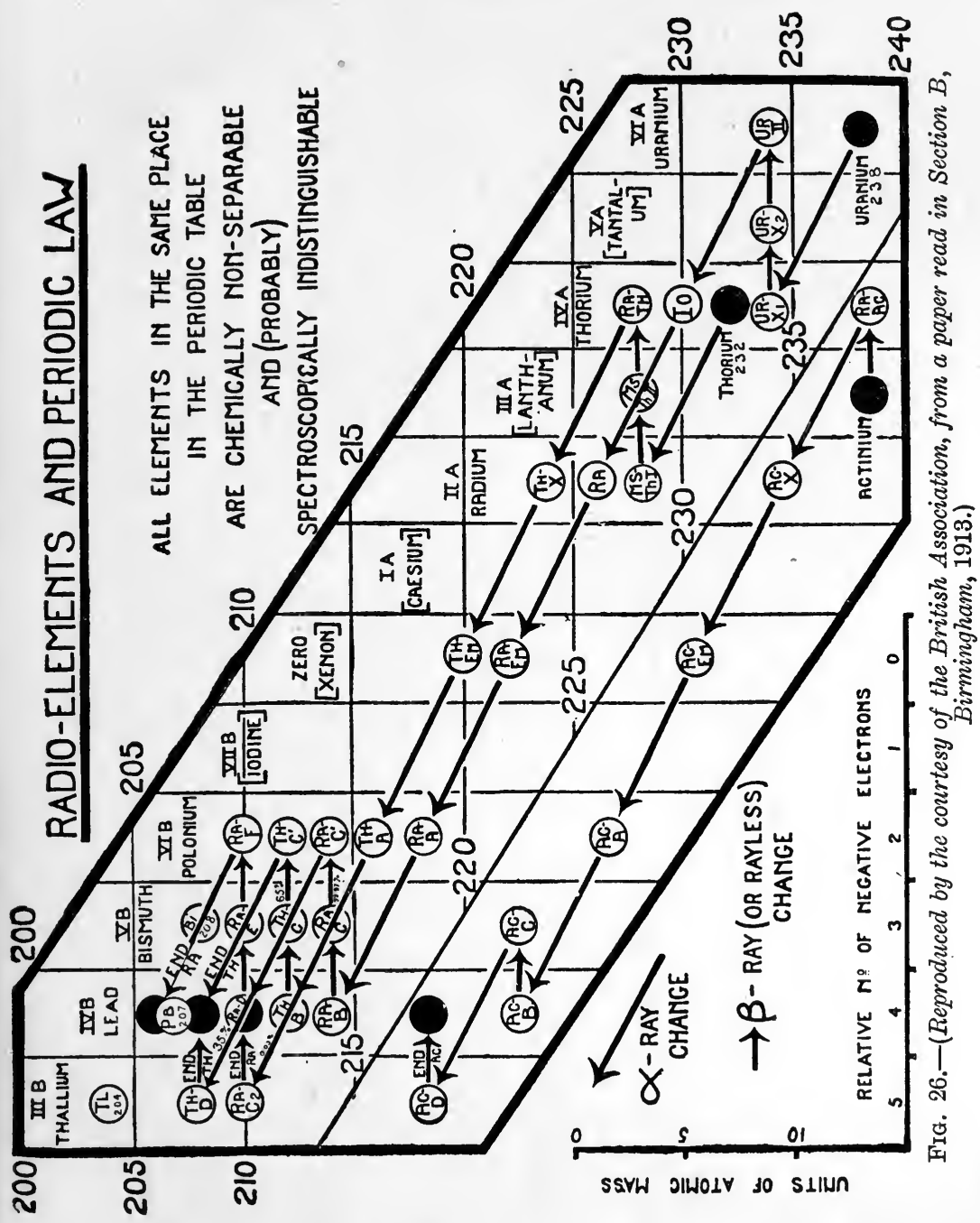

as Crookes ${ }^{1}$ suggested as far back at 1888 , composed of atoms

1 Crookes, Trans., 1888, 53, 487 ; see also Trans., 1889, 55, 257. The reader is advised to consult these papers. A mixture of two or more isotopes would correspond to Crookes' conception of a meta-element. 
of different atomic weights; and what we term the "atomic weight" may be merely an average value and not a true physical constant.*

Secondly, Soddy pointed out that when we go further up the Table in the vertical columns we come to the ordinary families of elements. In these families the differences in mass between similar elements are much greater than in the case of the isotopes; and instead of identity we get aualogy in chemical character between them. From this comparison, Soddy draws the conclusion that the chemical nature of matter is a function of two variables: mass and electrical character. The electrical content is the essential variable in the horizontal rows: whilst in the vertical columns the mass plays the preponderant part. Mass also enters into the problem of the stability of elements, and it may in some cases play a preponderant part from this standpoint.

\section{6. $\beta$-Rays and Ionic Charges.}

When a radio-element undergoes two successive $\beta$-ray changes, the net result is an increase of two units in its positive valency. Now an apparently similar process takes place when a divalent metallic ion becomes converted into a quadrivalent one; and it is natural to inquire whether the two processes are parallel or identical in nature. At Soddy's suggestion ${ }^{1}$ this problem was investigated by Fleck; ${ }^{2}$ and it was shown that the two cases are not identical but are merely parallel.

An examination of the diagram on p. 207 will show that the quadrivalent element uranium- $X_{1}$ loses two $\beta$-particles and is couverted into the hexavalent element uranium-2. Parallel to this we have the conversion of quadrivalent uranous salt, such as $\mathrm{UCl}_{4}$, into the hexavalent uranyl compound $\mathrm{UO}_{2} \mathrm{Cl}_{2}$. In both cases the parent substance gains two positive charges as a net result.

* It is noteworthy that in the same paper Crookes suggested that electricity might be one of the ultimate elements, a view subsequently modernized by Ramsay in his paper on "The Electron as an Element" (Trans., 1908, 93, 774).

1 Soddy, Nature, 1913, $92,399$.

2 Fleck, Trans., 1914, 100, 247. 
Now uranium-2 is isotopic with uranium-1 and is therefore chemically inseparable from the latter. If the $\beta$-ray change and the ordinary gain of two positive charges by an atom are identical processes, then the two starting substances also should be isotopic and inseparable by chemical means. In other words, a uranous salt and a salt of uranium- $\mathrm{X}_{1}$ ought to be chemically inseparable.

It is not necessary to select uranium- $X_{1}$ itself as the subject of the experiment; any of the five isotopes shown in the table will do equally well; and in practice thorium was chosen, since it is easily procurable.

Great precaution had to be taken that the uranous salt was not converted into the uranyl derivative during the process of separation; but by the use of a special air-free apparatus this was accomplished.

The results proved that when a mixture of uranous and thorium salts was fractionally precipitated, either by means of oxalic acid or by boiling off the excess of ammonium carbonate which was used to hold the salts in solution, a separation of the thorium from the uranium was readily obtained.

This result shows that uranous uranium and thorium are not isotopic with one another; and hence the conversion of uranium- $X_{1}$ into uranium- 2 by the loss of two $\beta$-particles is merely parallel to and not identical with the change which occurs when a uranous compound is converted into a uranyl derivative. Soddy deduces from these results that the electrons which are ejected during $\beta$-ray changes come from the nucleus of the atom, whilst mere alterations in valency affect the outer ring of electrons; and further, that there is no interchange of electrons between the nucleus and the shell in chemical or electrochemical changes.

\section{The Atomic Weight of Lead.}

An inspection of the chart on p. 207 will show that the end-products of the radioactive series, marked as black discs, all fall into Group IVb. of the Periodic System; and, since they only occupy one space in that Group, they are isotopic with one another. Closer inspection will reveal a still more interesting fact. 
If we take the atomic weight of radium as 226 , atomic weight of the product of radioactive change (passing through radium F) will be 206, since this series of changes involves the expulsion of five $a$-particles each of which has an atomic weight of four units. The end-product in the branch through radium $-\mathrm{C}_{2}$ will have an atomic weight of 210 , since only four a-particles are expelled during the changes.

Again, starting with thorium, which has an atomic weight of 232.4 , we find a succession of six a-ray changes in both branches of the chain, so that in each case the atomic weight of the end-product must be $208^{\circ} 4$.

With regard to actinium we are not on quite such sure ground; but the atomic weight of the end-product here must be either 208 or 212 .

Now the atomic weight of lead itself, as given in the International Table of Atomic Weights, is 207.1 ; and the question at once arises: Is ordinary lead a homogeneous element or is it a mixture of two isotopes? A mixture of the endproducts from uranium (through radium-F) and from thorium in equal proportions would have exactly the atomic weight which is required; and since the material is not radioactive and its two constituents would be inseparable from each other by chemical means, the results of atomic weight determination would work out exactly as in practice.

Soddy ${ }^{1}$ threw out a hint that the examination of the atomic weight of lead derived from minerals containing uranium and no thorium might differ from the atomic weight of lead found in other minerals containing thorium but no uranium; though he was careful to point out that the results might be obscured by other factors.

This line of research was taken up by several investigators. The first results were published by Soddy and Hyman ${ }^{2}$ who examined the lead derived from Ceylon thorite, a mineral containing 55 per cent. of thorium and 1 to 2 per cent. of uranium. The percentage of lead in the mineral is so small $(0.4$ per cent.) that it may be entirely of radioactive origin. Since the rate of change of uranium is two-and-a-half to three

* Hönigschmid's value is $225 \cdot 95$ (Monatsh., 1912, 33, 253).

1 Soddy, Annual Reports, 1913, X., 269-70.

2 Soddy and Hyman, Trans., 1914, 105, 1402. 
times that of thorium, the lead, if derived from both sources, should contain about ten parts of thorium-lead to one part of uranium-lead. On this calculation the atomic weight of lead from thorite ought to be rather higher than that of ordinary lead; and when the atomic weight was actually determined, it was found to be $207 \cdot 694^{*}$ as compared with $207 \cdot 2$ for ordinary lead. ${ }^{1}$ Photographs of the spectra of the two varieties of lead were found to be identical with the exception of one line, $4760 \cdot 1$, which was much stronger in the spectrum of ordinary lead than in that of thorite lead. ${ }^{2}$

Richards and Lembert ${ }^{3}$ then published a series of determinations of the atomic weights of lead from five different radioactive minerals and from two commercial, non-radioactive specimens of lead. They found that lead occurring in uraninite derived from North Carolina had an atomic weight of 206.40 whilst common lead had an atomic weight of $207 \cdot 15$. The leads from other minerals took up positions intermediate between these extremes. Richards and Wadsworth obtained the value of 206.08 for radioactive lead from Norwegian clèveite.

Another series of determinations is due to M. Curie. ${ }^{4}$ Lead from carnotite was found to have an atomic weight of 206.36 , whilst the metal from galena gave an atomic weight of $207 \cdot 01$.

Finally, Hönigschmid ${ }^{5}$ determined the atomic weight of lead from Joachimsthal pitchblende and found it to be $206 \cdot 405$.

Taking the whole of the results, ${ }^{6}$ it will be seen that the lowest figure is 206.08 and the highest is $207 \cdot 694$, common

* This is the corrected figure; see Soddy, Annual Reports, 1916, XIII., 247.

1 Baxter and Grover, J. Amer. Chem. Soc., 1915, 36, 356.

2 See also Hönigschmid and Horovitz, Monatsh., 1915, 36, 353; Merton, Proc. Roy. Soc., 1915, A, 91, 198; Siegbahn and Stenström, Compt. rend., 1917, 165, 428; Harkins and Aronberg, Proc. Nat. Acad. Sci., 1917, 3, 710, for other data with regard to the identity of the spectra of isotopes.

3 Richards and Lembert, J. Amer. Chem. Soc., 1914, 36, 1329; see also Richards and Wadsworth, J. Amer. Chem. Soc., 1916, 38, 2613.

- Maurice Curie, Compt. rend., 1914, 158, 1676.

3 Hönigschmid and Mlle. Horowitz, Compt. rend., 1914, 158, 1796 ; Monatsh., 1915, 36, 353.

- See also Soddy, Nature, 1915, 94, 615; Engineering, 1915, May 28 and October 1; Richards and Wadsworth, J. Amer. Chem. Soc., 1916, 38, 221, $1658,2613$. 
lead being $207 \cdot 2$. Since some of the results were obtained by investigators who have specialized in atomic weight determinations, no doubt can be entertained as to their accuracy; and it must be taken as : proved that the "atomic weight of lead" is not a physical constant, but differs according to the specimen chosen for examination.

Density determinations of various specimens of lead yielded further results of interest. Soddy and Hyman, working with the thorite lead mentioned above, found the density to be $\mathrm{D}_{4}{ }^{20}=11.376$ whereas the value for ordinary lead is 11.3415. Richards and Wadsworth found the density of lead from Norwegian clèveite to be $11 \cdot 273$. Other results were obtained by the same investigators. On calculating the atomic volumes for the various isotopic forms of lead from these measurements, it was found that all the isotopes had the same atomic volume. The melting-points of isotopes appear to be identical. ${ }^{1}$

The importance of the foregoing results requires but little emphasis. Clearly they strike at the very root of our ordinary chemical conceptions; and, since they are found in the case of non-radioactive materials, they cannot be brushed aside as forming part of the abnormalities which characterize the radio-elements. They form the most conclusive evidence of the correctness of Soddy's theory of the isotopes from the purely chemical side, as the proof of the existence of different forms of lead can now be based entirely upon chemical investigation, independent entirely of radioactivity measurements; and with this advance we enter upon the field of the normal elements themselves.

\section{Other Cases of Isotopy.}

If it be assumed that isotopic elements are identical in chemical properties, but different in atomic mass, it is evident that the only method of separating one isotope from another will depend upon the employment of some process which is influenced by the mass factor. Positive ray analysis appears to supply the needed implement; since it is essentially a method of sifting heavy atoms from lighter companions.

1 Lembert, Zeitsch. Elektrochem., 1920, 26, 59. 
When the positive ray method was applied to the fraction of liquid air which contains the heavier gases, parabolas were produced corresponding to neon, argon, krypton, and xenon; which was just what might have been expected. The photograph taken with the lighter constituents, on the other hand, yielded something quite new. ${ }^{1}$ In addition to the lines corresponding to helium and to neon with one and two charges, there was a line which pointed to the presence of an element having the atomic weight 22 . This might be ascribed to a doubly-charged molecule of carbon dioxide $(44 \div 2)$; but when all the carbon dioxide had apparently* been removed from the gas this line still persisted. The line is fainter than the neon line, showing that the percentage of the " 22 " gas in the atmosphere must be less than that of neon.

There is another possible explanation of the occurrence of the line. If neon combined with two atoms of hydrogen to form a compound $\mathrm{H}_{2} \mathrm{Ne}$, the results would be explicable. There is, apparently, evidence which weakens this suggestion. Another line corresponding to the value 11 has been detected; and this implies the presence of the same substance with a double charge. Now doubly-charged atoms are known; but up to the present no molecule has been found to carry more than a single charge. Thus we have to choose between accepting an atom with a weight of 22 or a molecule of $\mathrm{H}_{2} \mathrm{Ne}$ which behaves differently from all other molecules hitherto examined.

Thomson suggests that neon has a close ally, termed metaneon, with an atomic weight of 22. Attempts have been made to separate the two gases from one another by diffusion, and it is stated that the result was a separation of atmospheric neon into two fractions; but this has been contradicted by another investigator.

The spectra of two diffusion fractions showed no abnormality; but this may be explicable either on the assumption that the spectra of neon and metaneon are identical, or by assuming that the quantity of metaneon present was so small that its spectrum was swamped by the brilliant neon spectrum.

1 Thomson, Rays of Positive Electricity, p. 112 (1913).

* Compare, however, the observation of Collie and H. S. Patterson (Proc., $1913,29,217$ ) that carbon compounds are retained in vacuum tubes under conditions which normally might be supposed to preclude their existence. 
When the positive ray method is applied to the study of other gaseous elements, ${ }^{1}$ equally interesting results are obtained. Helium and argon give parabolas which accord with the atomic weights 4 and 40 ; and no trace of subsidiary parabolas was obtained. Krypton, on the other hand, seems to yield no less than six parabolas which are ascribed to constituents having the atomic weights : $78,80,82,83,84$, and 86 . Xenon produces five parabolas, which would correspond to the atomic weights : 128,130 , 131,133 , and 135. Nitrogen appears to be composed of homogeneous atoms; but chlorine leaves parabolic traces on the plate which accord with the atomic weights : 35 and 37 . The results in the case of mercury are said to support the view that this element contains atoms with weights of 202 and 204 as well as material which lies between 197 and 200 .

Thus, if the positive ray method be accepted, it seems evident that isotopy is not confined to the direct environment of the radioactive elements, but is a general phenomenon; and these results appear to favour a return to a modified form of Prout's hypothesis, since they point to the possibility that all elements are mixtures of atoms from each other in atomic weight by complete units.

\section{Conclusion.}

Before closing this chapter, some account must be given of the bearing of the isotopic theory upon the general question of the elements. It is not claiming too much to state that the isotopes furnish us with a means of defining the difference between one element and another to a degree which ten years ago might have been thought impossible. Until the discovery of isotopy, it was assumed by the majority of chemists that chemical analysis did actually segregate matter into homogeneous classes if it were carried far enough; and those who held Crookes' ideas on the possibility of meta-elements were regarded as somewhat eccentric. It is true that in the field to which Crookes originally applied the meta-element viewthe rare earths-the conception has not been substantiated;

1 Aston, Nature, 1919, 104, 393; 1920, 105, 8; Phil. Mag., 1920, 39, 449. 
but the isotopes are exactly in agreement with the meta-element definition.

We now know that chemical methods of separation are at the best superficial. To use a crude analogy, the local surveyor may tell us that three houses are absolutely identical in every way; but when we call in the local registrar he will be able to state that one house is inhabited by a bachelor, the next one by an old maid, whilst the third contains a family of seven. Chemical analysis is represented by the surveyor, whilst radioactivity measurements play the part of the registrar and tell us what the internal economy of the house may be.

According to Soddy, ${ }^{1}$ it may now be taken as proved that so long as the net charge on the atomic nucleus is the same, the element will show the definite chemical and physicochemical character associated with one or other of the ninetytwo places of the Periodic Table, quite independently of the nature and constitution of the nucleus. Its light spectrum and its X-ray spectrum will be identical with that of any other element fulfilling the same conditions of net charge. But since the net charge on the nucleus depends not on the absolute but only on the relative numbers of positive and negative charges associated with it, it is clear that two atoms may possess identical chemical properties and yet differ in mass. For example, one atom may contain three negative and five positive charges; whilst another contains one negative and three positive charges. In both cases the net charge will be two positive; and the chemical characters of the two atoms will be identical; but their masses will be different, since it appears to be a function of the number of positive charges present.

Even this does not entirely state the case ; for it is possible that two atoms may contain the same net nuclear charge and the same absolute number of opposite charges and yet these two atoms may differ from one another in the structure of their nuclei. Among the radio-elements cases of this kind actually occur. For example, in the following scheme, it will be seen that radium-C may disintegrate in either of two ways :-

1 Soddy, Annual Reports, 1916, XIII., 245. 


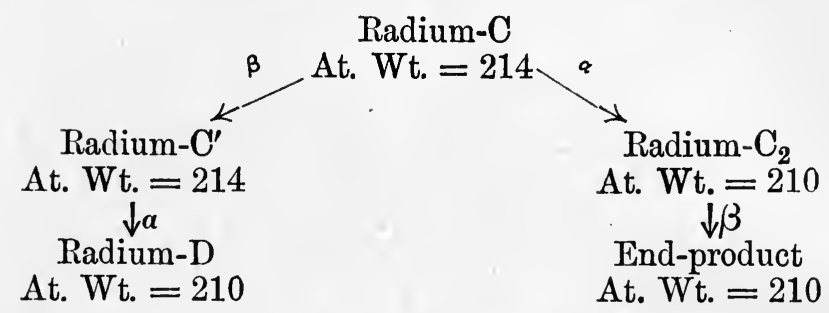

In the one case, an $a$-particle is ejected first and then a $\beta$-ray is subsequently thrown off; in the second case, the order is inverted and the expulsion of the $\beta$-ray precedes that of the $a$-particle. The two end-products obviously have resulted from the same substance, radium-C, by the loss of exactly the same number of charges in each case ; but the two end-products are not identical : for radium-D continues to disintegrate, yielding radium-E, whilst the other element with atomic weight 210 has apparently reached the end of its active disintegration.

This example illustrates what might be called a finer degree of isotopy, since the two elements are alike in chemical character, spectrum, and atomic mass, and yet, as the difference in radioactivity shows, they are not identical.

Mass and chemical properties alone, then, cannot be taken as criteria of elemental homogeneity. It is true that we cannot voluntarily disintegrate "chemical" elements; but even at present we can "manufacture" them. If we mix together equimolecular quantities of two forms of lead, one having an atomic weight of 206.4 and the other with an atomic weight of $207 \cdot 7$, any chemist to whom we gave the mixture for analysis would find that the element lead was present and that its atomic weight was $207 \cdot 05$. Thus the mixture is a "chemical" element with an atomic weight which characterizes it; and it cannot be separated by chemical means into anything simpler.

Paneth ${ }^{1}$ suggests that the name "element" should be confined to those substances which cannot be separated by chemical processes. All elements isotopic with one another would then be regarded as one "element." The light spectra and the $\mathrm{X}$-ray spectra would thus still remain as criteria of chemical elements. When an element is composed of only one kind of

1 Paneth, Zeitsch. physikal. Chem., 1916, 91, 171; ibid., 1918, 93, 86, 677 ; Wegscheider, ibid., 92, 741. 
atom, it would be termed a "pure" element; whilst elements containing more than one type of atom would be regarded as " mixed" elements. Time alone can show whether this system of nomenclature is sufficiently elastic to cope with the additions to our knowledge which are being made in the field of radioactivity.

Some attempts ${ }^{1}$ have been made to,devise methods whereby two isotopes might be separated from each other: but with the exception of a trial of centrifuging ${ }^{2}$ these suggestions are theoretical only, and positive ray analysis is at present our only experimental means of sifting homogeneous atoms from a heterogeneous mixture.

1 Lindemann and Aston, Phil. Mag., 1919, 37, 523; Fleck, Nature, 1920, 104, 565; Merton and Hartley, ibid., 105, 104 ; Chapman, ibid., 487; Soddy, ibid., 516.

2 Joly and Poole, Phil. Mag., 1920, 39, 372. 


\section{CHAPTER XVIII}

THE ELEMENTS

\section{Isotopes and Isobares.}

INVESTIGATIONS in the field of radioactivity have broadened our outlook upon many of the basic problems of chemistry; but, at the same time, it must be confessed that they have increased our difficulties in certain directions, especially in respect to the conception of elements.

The following definition of an element appears to cover practically all the ground: "An element is a form of matter which has (1) a definite atomic weight; (2) a definite spectrum, whether it be an emission or an absorption spectrum; (3) a definite valency or electrical charge; (4) a definite series of chemical properties; (5) a resistance to any ordinary chemical reaction such that no decomposition of the material into anything simpler is possible; and, in certain cases, (6) a definite series of radioactive properties." If any form of matter agrees with all these criteria, we are justified in assuming that it is chemically elemental in character.

Now let us return for a moment to some of the facts which were mentioned in the last chapter. It was there made clear that change in the radioactive elements may take place in either of two ways: (1) by the expulsion of an a-particle; or (2) by the ejection of a $\beta$-ray. The alteration in properties which ensues when an $a$-particle is expelled results from two causes : a loss of atomic weight of four units and a lessening of the positive charge on the atom by two units; so that the net result is to bring the product of the change into a position in the Periodic Table two places to the left of the parent element. The new substance thus produced conforms with all the criteria of an element which have been given above. Let 
us turn now to the $\beta$-ray change. It results in the removal of one negative charge from the parent atom; so that the product, while retaining the same atomic weight, is shifted into the next column to the right in the Periodic Table. Here again the new form of matter produced is elemental in type. As we have already seen, an $a$-ray change followed by two successive $\beta$-ray changes results in the production of an element whose atomic weight is four units less than that of the parent substance; and this new element occupies a position in the same column of the Periodic Table as its parent did.

Let us look a little more closely into the matter; and choose as a concrete example the transformations which begin with thorium and end with radiothorium-

Group IIa.

Group IIIa.

Mesothorium-1 $\underset{\text { change }}{\stackrel{\beta \text {-ray }}{\longrightarrow}}$ Mesothorium-2 $\underset{\text { change }}{\stackrel{\beta \text {-ray }}{\longrightarrow}}$ Radiothorium

228

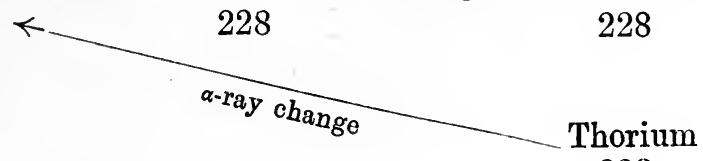

232

An examination of the scheme above will show that we can distinguish between two classes of radioactive elements.

In the first class are elements such as thorium and radiothorium which can be distinguished from one another by their different radioactive properties, but which are inseparable from one another by purely chemical means. They differ from one another in atomic weight; but owing to the identity of their chemical properties they must be placed in the same space in the Periodic Table. On this account Soddy named them isotopes (from isos, equal, and topos, a place).

In the second class, to which special attention must now be drawn, we may place elements such as mesothorium-1, mesothorium-2 and radiothorium. These elements are identical in atomic weight but differ from each other in chemical character; so that they are separable from one another by chemical reagents. Since their atomic weights are identical, the name isobares has been suggested for them (from isos equal, and baros, weight). Isobaric elements may be defined as 
elements which possess the same atomic weight but which differ from each other in chemical properties. ${ }^{1}$

Thus, as Soddy ${ }^{2}$ has pointed out, the atoms of the elements can now be grouped under four heads-

\begin{tabular}{|l|c|c|c|}
\hline & $\begin{array}{c}\text { Atomic } \\
\text { Weights. }\end{array}$ & $\begin{array}{c}\text { Places in } \\
\text { table. }\end{array}$ & \multicolumn{1}{|c|}{ Examples. } \\
\hline $\begin{array}{l}\text { Heterobaric heterotopes } \\
\begin{array}{l}\text { Heterobaric isotopes } \\
\text { Isobaric heterotopes } \\
\text { Isobaric isotopes }\end{array}\end{array}$ & $\begin{array}{l}\text { Different } \\
\text { Different } \\
\text { Same }\end{array}$ & $\begin{array}{c}\text { Different } \\
\text { Same } \\
\text { Different } \\
\text { Same }\end{array}$ & $\begin{array}{l}\text { Lame } \\
\text { Sathium and chlorine } \\
\text { Thorium and ionium } \\
\text { Mesothorium-1 and meso- } \\
\text { thorium-2 } \\
\text { Radium-C }\end{array}$ \\
\hline
\end{tabular}

So far, we have concerned ourselves principally with the radio-elements ; but the question now suggests itself : Are the isobaric elements confined to the radioactive group; or do we find any example of this class outside the last line in the Periodic Table? If isobares exist apart from the radio-elements, they must fufil all the conditions which were laid down earlier in this chapter as tests for elemental character.

Substances which fulfil the first five * conditions are actually known; and it is probably only ingrained conservatism which has hindered a recognition of them at an earlier stage. Ever since the days of alchemy, the transmutation of the elements has been the most entrancing object of research; but at the end of last century it had gradually become one of the dogmas of conservative chemists that transmutation was impossible. Then came the discovery of radioactive changes; and transmutation was actually found to be occurring continually and spontaneously in one particular series of substances. Still, however, the idea of transmutation was confined to a process whereby the atomic weight of an element was altered. The old attempts to convert lead into gold had left their legacy behind them; and the only change recognized as "transmutation" was one in which an alteration in atomic weight occurred.

The researches which led to the formulation of Soddy's

1 A. W. Stewart, Phil. Mag., 1918, 36, 326.

2 Soddy, Trans., 1919, 115, 1.

* Since they are not radioactive bodies the sixth criterion is not applicable in their case. 
Law have altered our ideas in this field. The conversion of, say, mesothorium-1 into mesothorium-2 is just as much a case of "transmutation" as the change of radium into niton is. It is now impossible to deny that "transmutation" may take either of two forms : (1) a change in atomic weight and (2) a change in chemical character unaccompanied by any alteration in atomic weight. Mesothorium-1 and mesothorium-2 are quite obviously different elements, yet each has the same atomic weight: 228. The fact that this type of transmutation among the radio-elements is spontaneous and irreversible has apparently blinded chemical investigators to some obvious deductions which can be drawn from the behaviour of betterknown materials; for no attempt has hitherto been made to apply analogous reasoning to elements outside the radioactive group.

Let us select as an illustration the case of ferrous and ferric iron; and let us place the properties of the two "irons" in parallel columns, using the elemental criteria given above in each caso-

\begin{tabular}{|c|c|c|}
\hline & Ferrous Iron. & Ferric Iron. \\
\hline $\begin{array}{l}\text { Atomic weight } \\
\text { Absorption spectra } \\
\text { Valency } \\
\text { Chemical properties : } \\
\text { Resistance to decomposition }\end{array}$ & $\begin{array}{c}55 \cdot 84 \\
\text { Different from ferric } \\
\text { Divalent } \\
\text { Resembles magnesium } \\
\text { Complete }\end{array}$ & $\begin{array}{c}55 \cdot 84 \\
\text { Different from ferrous } \\
\text { Trivalent } \\
\text { Resembles aluminium } \\
\text { Complete }\end{array}$ \\
\hline
\end{tabular}

An examination of the above table shows that ferrous and ferric iron fulfil all the criteria which we imposed in order to distinguish one element from its isobare. The atomic weights of the two bodies are identical, so they are isobaric with one another. The absorption spectra of their salts are different even to the naked eye. (It is impossible to determine whether or not their emission spectra are different, owing to the possibility of transmutation taking place under the abnormal conditions necessary to produce emission spectra.) Their valencies are different from each other, since ferrous iron unites with two chlorine atoms whilst ferric iron combines with three. In their chemical properties there is little resemblance between them: for ferrous iron in its reactions closely resembles 
magnesium; whilst ferric iron is much more akin to aluminium. Neither can be broken down by ordinary chemical reactions into anything simpler.

It is worth while to examine the parallelism which exists between ferrous and ferric iron on the one hand and, say, mesothorium-1 and mesothorium-2 on the other; though it must be carefully borne in mind that the two cases are not identical.* None the less the closeness of the parallelism suggests that, although the origins of the phenomena are different, there is marked kinship between the operations of the two transmutational processes which are at work.

Mesothorium-1 and ferrous iron both exhibit properties which are shown by elements in Group II. of the Periodic Table. Both form salts of the type $\mathrm{RCl}_{2}, \mathrm{RSO}_{4}$, etc. Both are convertible into a form of matter which possesses an extra positive charge-mesothorium-2 in the one case and ferric iron in the other. On the other hand, mesothorium-2 and ferric iron are both trivalent; and each of them forms salts of the type $\mathrm{RCl}_{3}$.

The obvious difference between the two pairs lies in the fact that we can convert ferrous iron into ferric iron or vice versa $\hat{\imath}$ at will; whilst the conversion of mesothorium-1 into mesothorium-2 proceeds spontaneously and irreversibly as far as our present means go. In both cases, negative electric charges are removed from the divalent atoms; but in the one case the charge is taken from a certain part of the atomic structure whilst in the other case it comes from a different portion of the atom.

Even the spontaneity of the $\beta$-ray change finds its parallel among certain of the non-radioactive elements. Thus when the chloride of monovalent indium is dissolved in water it is spontaneously converted into metallic indium and the chloride of trivalent indium-

$$
3 \mathrm{InCl}=\mathrm{InCl}_{3}+2 \mathrm{In}
$$

Reduced to its essentials, this change corresponds to the loss of two negative charges from each of the monovalent indium atoms; and no external forces are required in order to bring

* For the evidence of this, see p. 208. 
about the phenomenon. The case of indium is by no means an isolated one ; for other elements exhibit a similar behaviour. *

Yet a further parallelism between the $\beta$-ray change and the conversion of an ion into one of a higher valency may be adduced here. In several cases, elements are found to exist in monovalent and trivalent forms, or in the divalent and quadrivalent condition only, instead of forming a complete series of mono-, di-, tri-, and quadrivalent varieties. Thus thallium forms the chlorides $\mathrm{TICl}$ and $\mathrm{TlCl}_{3}$, but does not yield the intermediate $\mathrm{TlCl}_{2}$. Similarly, germanium forms $\mathrm{GeCl}_{2}$ and $\mathrm{GeCl}_{4}$, but not $\mathrm{GeCl}_{3}$. It may be asked why these intermediate forms are not isolated as we remove electric charges from the substances of lower valency.

An examination of the state of affairs in the case of the radio-elements may throw some light upon this point. The conversion of $\mathrm{GeCl}_{2}$ into $\mathrm{GeCl}_{4}$ is paralleled by two successive $\beta$-ray changes in the radio-elements; and in the following table the results of such successive changes are given. The cases have been selected in which no disturbing factor in the shape of an alternative $a$-ray change occurs. The figures give the average life of the elements.
Group $x \underset{\text { change }}{\stackrel{\beta \text {-ray }}{\longrightarrow}}$ Group $(x+1) \underset{\text { change }}{\stackrel{\beta \text {-ray }}{\longrightarrow}}$ Group $(x+2)$
Uranium $\mathrm{X}_{1}$
Uranium $\mathrm{X}_{2}$
$35 \cdot 5$ days
Mesothorium-1
1.65 minutes
$7 \cdot 9$ years
Radium D
24 years

Mesothorium
8.9 hours
Radium E
7.20 days
Uranium II
$3 \times 10^{6}$ years
Radiothorium
$2 \cdot 01$ years
Radium F
196 days

It will be seen that the intermediate product in the double $\beta$-ray change has an average life very much shorter than those of the parent or the disintegration product. If the same reasoning holds good in the case of the salts of, say, thallium, we should expect to find that when monovalent thallium loses an electric charge and passes into divalent thallium, the latter

* I am indebted to Dr. Smiles for calling my attention to the fact that a reaction of this type is the most general one in chemistry. It finds its parallel in organic chemistry in such reactions as the conversion of benzaldehyde into benzoic acid and benzyl alcohol by treatment with alkali. 
substance loses a second electric charge readily and passes almost immediately into trivalent thallium, just as mesothorium-2 soon breaks down to radiothorium. The parallel between the two cases is thus closer than appears at first sight.

The question of isobarism may be considered from another point of view. Hittorf observed from E.M.F. measurements that chromium ${ }^{1}$ existed in no less than three forms; and he stated that "in the three conditions the metal exhibits differences of properties such as have been found only in different metals." In the inactive state, chromium behaves like a noble metal and stands at the end of the electrochemical series beside platinum. In the second state it ranks immediately behind zinc in the series. The third state brings it into a position intermediate between the two others. In the inactive condition it refuses to unite with iodine even when the latter element is nascent; whilst in the active condition it is capable of displacing hydrogen from hydriodic acid. ${ }^{2}$

Again, just as two isotopes can be mixed in order to produce a new substance having an atomic weight dependent upon the proportions in which the two components are present, so two forms of chromium can co-exist and give rise to a material which has an E.M.F. intermediate between those of the two forms when alone. ${ }^{3}$

For the sake of clearness, it may be well to summarize the matter in a few words. It is now proved by experimental evidence that two elements having totally different chemical and radioactive properties may have the same atomic weight. This is termed isobarism. Parallel to this but not identical with it, is the case of an element exhibiting two or more degrees of valency. This might be termed pseudo-isobarism. Between isobarism and pseudo-isobarism there are certain points of resemblance which appear to point to a parallelism between the two phenomena in their ultimate origins.

1 Hittorf, Zeitsch. physikal. Chem., 1898, 25, 729; 1899, 30, 481; 1900, 43, 885. Compare also the cases of iron, Hittorf, ibid., 1900, 43, 385; Müller and Koenigsberger, Physikal. Zeitsch., 1904, 14, 413, 797 ; Müller, Zeitsch. physikal. Chem., 1904, 48, 585; Finkelstein, ibid., 1902, 39, 91; cobalt and nickel, Hittorf, ibid., 1900, 43, 385.

2 Hittorf, Zeitsch. physikal Chem., 1898, 25, 748.

${ }^{3}$ Hittorf, ibid., 1899, 30, 505. 


\section{The Structure of the Atom.}

The problem of atomic structure has frequently been examined in recent years; and in this place only the barest outline of the subject can be given. For further information the reader is referred to other works. ${ }^{1}$

Kelvin ${ }^{2}$ suggested a model atom in which negative particles were embedded in a uniform sphere of positive electricity having the volume of the atom. This view has been further developed in detail by J. J. Thomson. ${ }^{3}$ From the analogy of Mayer's Magnets, ${ }^{*}$ Thomson has deduced that under the conditions of the hypothetical atom, the electrons would group themselves in certain ring-systems, the nature of the rings being governed by the number of electrons present. The periodicity of properties among the elements is explained on this basis by the periodic occurrence of similar rings in the arrangement as the system grows more complex.

This view served to account for a certain number of the known facts with regard to chemical valency; but some of Thomson's deductions failed to satisfy the ordinary chemist.

Experimental work by Rutherford brought the matter into a new stage. When a stream of $a$-particles is allowed to pass through a thin sheet of some solid substance, it is found that some of them are stopped entirely or deflected through an angle from their original paths. This is called "scattering." Now calculation shows that the chance of the particles passing through the interstices between the molecules of the sheet is a very small one; whence it must be deduced that these $a$-particles in many cases actually pass through the atoms which make up the sheet. On this view, the deflections

1 J. J. Thomson, The Corpuscular Theory of Matter (1907); Rutherford, Radioactive Substances and their Radiations (1913); Richardson, The Electron Theory of Matter (1914) ; Millikan, The Electron (1917).

2 Kelvin, Phil. Mag., 1902, 3, 257.

3 J. J. Thomson, Phil. Mag., 1904, 7, 237, and The Corpuscular Theory of Matter.

* Mayer's Magnets are magnetized needles with cork floats which are so arranged that when the magnets are placed in water all the like poles float above the surface. When a large magnet is placed with its opposite pole over the centre of the vessel in which the needles float, the latter arrange themselves into a series of fixed groupings, according to the number of magnets afloat at one time. 
observed experimentally must be brought about not by atomic collisions but by the action of electrical charges within the atoms of the sheet.

Thomson ${ }^{1}$ believes that the total deflection observed is actually the resultant of a series of small deviations brought about by the successive actions of a series of small charges within the atom. Rutherford, ${ }^{2}$ on the other hand, holds that certain cases of this "scattering" can be explained only on the assumption that the deflections are due to the single operation of a large concentrated charge within the atom. The experimental evidence appears to favour Rutherford's view. ${ }^{3}$

In order to explain this "scattering" of a-particles, Rutherford ${ }^{4}$ suggests that the atom consists of a concentrated positive charge surrounded by a zone of electrons sufficient in number to render the system as a whole neutral. For example, the uranium atom consists of a central positive charge surrounded by a number of concentric rings of negative electrons in rapid motion, the outer ring being comparable in diameter to the diameter of the atom. Practically the whole positive charge and mass of the atorn is confined within a sphere of radius not greater than $10^{-12} \mathrm{c.m}$. The positively charged centre of the atom is supposed to be a complicated moving system consisting in part of charged helium and hydrogen atoms. The assumption is made that at very small distances the positively charged particles attract instead of repelling one another.*

Another suggestion has been put forward by Bohr ${ }^{5}$ which also assumes that negative electrons are circulating in orbits around a positive nucleus. The pecularity of the Bohr atom,

1 J. J. Thomson, Camb. Phil. Soc. Proc., 1910, 15, 465.

2 Rutherford, Phil. Mag., 1911, 21, 669.

3 Geiger and Marsden, Proc. Roy. Soc., 1909, A, 82,495; Geiger, ibid., 1910, A, 83, 492.

4 Rutherford, Radioactive Substances, 1913, p. 619 ff.

5 Bohr, Phil. Mag., 1913, 26, 1, 476, 857; 1915, 29, 332 ; 30, 394.

* This assumption might form the basis of an interesting speculation. Mass is always regarded as a "signless" quantity-i.e. we do not take the possibility of negative mass into consideration. But under the abnormal conditions assumed by Rutherford, the possibility of negative mass suggests itself, and the atomic weight might be regarded as the algebraic sum of positive and negative masses within the atom. If the positive mass exactly equalled the negative mass, then something akin to the luminiferous ether would result. 
however, lies in the fact that the electrons are assumed to "jump" from one orbit to another; and that during this "jump," radiation takes place. Bohr's calculations agree very well with spectral results in the case of hydrogen; but they are unsatisfactory when applied to more complicated atoms. ${ }^{1}$

Bohr's atom depends upon a fusion of Newtonian dynamics with the quantum theory; but Nicholson has avoided this by assuming that the vibrations causing spectral lines occur in a plane perpendicular to that in which the electrons revolve about the centre of the atom. This theory has received strong support from stellar spectroscopic evidence; for lines predicted by Nicholson have been detected in the Iick Observatory photographs when special attention had been called to their position as a result of Nicholson's work, though they had escaped notice in previous examinations owing to their faintness.

Nicholson's work has brought again into prominence the possibility of elements existing which are unknown upon the Earth and for which there are no places in our present Periodic Table unless, as van den Broek $^{2}$ suggests, they are isotopic with hydrogen. If this view be correct, then the isotopes of hydrogen would be the "proto-elements" : coronium, nebulium, and proto-fluorine. But if the spectra of two isotopes are identical, as seems to be the case, then "protofluorine" would have the same spectrum as fluorine, which is, of course, not correct.

Other attempts have been made to construct atoms by the combination of lighter elements $;^{3}$ but they cannot be dealt with here.

Most of the foregoing views have been based chiefly upon physical evidence; but other attempts have been made which treat of the chemical side.

According to G. N. Lewis, ${ }^{4}$ every atom consists of a kernel and a shell. The kernel remains unaffected by chemical changes

1 Nicholson, Phil. Mag., 1914, 27, 541; 28, 90.

2 van den Broek, Phil. Mag., 1914, 28, 630.

3 Nicholson, Phil. Mag., 1911, 22, 864; van den Broek, Physikal. Zeitsch., 1911, 12, 490 ; 1916, 17, 260 ; Harkins and Wilson, J. Amer. Chem. Soc., 1915, 37, 1396; Phil. Mag., 1915, 30, 723.

4 G. N. Lewis, J. Amer. Chem. Soc., 1916, 38, 762 ; Science, 1917, 46, 297 ; cf. J. Amer. Chem. Soc., 1913, 35, 1448; Bray and Branch, ibid., 1913, 35, 1440. 
and possesses an excess of positive charges numerically equal to the ordinal number of the Group in the Periodic Table to which the atom belongs. The number of negative electrons in the shell may vary during chemical change between 0 and 8 . The atom tends to hold an even number of electrons in its shell ; and especially to hold eight electrons, which are normally arranged at the eight corners of a cube. Lewis assumes that two atomic shells are mutually interpenetrable; and that the electrons may pass, with readiness from one position of the outer shell to another. The crux of his hypothesis is that one electron may simultaneously occupy the corners of two shells, if the corners are brought into superposition. By a further process of superposition a second pair of corners may come into cuincidence; and thus a second electron will become common to the two atoms represented by the cubes. In this way, single and double bonds are represented. Further, it is possible to distinguish between different types of bonds; and thus differences in reactivity may be expressed. Finally, if it be assumed that the electrons in the shell are free to move along the edges of the cube towards the middle of the edge, an arrangement can be obtained which corresponds to the van't Hoff tetrahedric arrangement of the carbon atom.

Noyes ${ }^{1}$ has put forward a dynamic conception of the atom. He assumes that atoms are built up from two different sets of electrons: (1) those attached to a positive nucleus, and (2) valency electrons which are free to wander from atom to atom. When two atoms capable of interacting are brought together, Noyes suggests that a valency electron which is rotating round the positive nucleus of the first atom may find a positive nucleus of the second atom so close to it that it may proceed to describe an orbit round the positive nuclei of both atoms. During the portion of the orbit which lies within the second atom, that atom would become wholly negative whilst the first atom would be positive. During the other portion of the orbit each atom would be electrically neutral and the atoms might fall apart. In ionization, the electron would revolve entirely about the nucleus of the negative atom, leaving the other atom positive. If it be assumed that there are four, or eight, positive nuclei in the carbon atom, the hypothesis serv's to

1 Noyes, J. Amer. Chem. Soc., 1917, 39, 879. 
aecount for the tetrahedric grouping which is found in optically active carbon compounds.

A more detailed hypothesis has been suggested by Stewart. ${ }^{1}$ On his view, the atom is assumed to be made up of three separate regions: (1) a core of negative electrons; (2) an intermediate zone occupied normally by positive electrons but containing also, in the case of the radio-elements, certain negative electrons; and, finally, (3) an external region occupied by negative electrons. The orbits of the electrons in the two inner zones are assumed to be approximately circular; whilst those of the external electrons are supposed to be extremely elongated ellipses, similar to the paths of comets in the Solar System.*

The negative electrons in the core of the atom are assumed to be travelling at high velocities; and since charges moving at high speeds are difficult to deviate from their normal orbit by external forces, this serves to account for the fact that ordinary reactions fail to affect the intimate chemical structure of atoms.

In the radio-elements, the core is assumed to be the point of origin of the $\beta$-rays; whilst the intermediate zone generates the a-particles. The atomic number corresponds to the surplus of positive over negative charges in the two inner zones together. $^{2}$ The radius of the positive zone is limited, by Rutherford's work, to approximately $10^{-12} \mathrm{~cm}$.

With regard to the conditions of attraction in the system, the negative charge in the core forms a centre around which the positive electrons can revolve; whilst the two inner zones together, having a surplus positive charge, will serve to retain the "cometary" electrons in their orbits.

The expulsion of the a-particle is assumed to result from the following process. In the intermediate positive zone of the radio-elements there are, in addition to the positive electrons, certain negative electrons; and the two types of charge are linked into "sun-and-planet" systems of one

1 A. W. Stewart, Phil. Mag., 1918, 36, 326.

2 Cf. Soddy, The Chemistry of the Radio-elements, ii., 40 (1914).

* It has been shown mathematically by Jackson, Phil. Mag., 1919, 38, 256 , that this system is unstable on the Newtonian assumptions. The existence of Rutherford's positive nucleus would be equally impossible on the same premises. 
negative and two positive charges which revolve as a whole around the central negative core of the atom. In the case of the uranium atom, which ejects eight $a$-particles in the course of its disintegrations, there will be at least sixteen such systems. When, by a crossing of orbits or by mutual disturbance, two such systems collide within the atom, a mass of four positive and two negative charges will be formed-i.e. a helium atom with two extra positive charges; and this mass will be ejected from the atom as a result of the collision, thus forming the $a$-particle. In this way the theory provides for the production of helium from the radio-elements without the necessity of postulating the actual presence of helium atoms, as such, within the atomic structure.*

The chief feature of Stewart's atom, however, is the conception of the "cometary" orbits of the external electrons. These electrons are assumed to be the class involved in chemical changes. Now during alterations in valency by means of ordinary chemical réactions, two phenomena are noticeable: first, the comparative ease with which the change is brought about in certain cases; and, second, the marked effect upon the chemical and physical character of the element which is produced by the alteration in valency. A satisfactory model atom must account for both these facts; and none of the "physical" atoms appear to throw much light upon the subject. Stewart's atom seems to succeed at this point.

When the electrons in the "cometary" paths are in aphelion to the centre of the atom, they will be travelling slowly in their orbits and they will also be only weakly attached to that centre. Thus at this particular region in their path they can be readily removed from or replaced in their orbits; which accounts for the comparative ease with which changes of valency and chemical reactions take place. Secondly, when these electrons reach perihelion with regard to the centre of the system, they will pass very close to the positive zone; and they will then affect the positive electrons in that zone from the outside just as the negative electrons of the core affect it from the inside. A change in the number

* If the neon discovered in certain mineral springs eventually proves to be of radioactive origin, an extension of the above hypothesis would be necessary. 
of electrons in the "cometary" orbits will therefore influence the positive zone just as it would be affected by a change of the number of electrons in the core. The two effects are not, however, identical: for the influence of the core electrons is constant; whereas that of the "cometary" electrons is temporary and periodic. Thus a change in the number of the "core" electrons produces a permanent effect and alters the atomic number; whilst a change in the number of "cometary" electrons tends in the same direction, but lacks permanence. The latter influence is therefore sulficient only to produce a change in chemical character approaching to-but notidentical with-a change in atomic number. For example, the conversion of ferric iron into ferrous iron yields a substance which has many of the properties of magnesium but which is not isotopic with that element. Similarly, the conversion of hexavalent into quadrivalent uranium produces a material very closely resembling thorium but not isotopic with it; so that Stewart's atom furnishes a simple explanation of the work of Soddy and Fleck.*

This model atom also suggests a solution of the rare earth problem, though it must be admitted that the matter is purely speculative. In order to account for the effects of the $a$-and $\beta$-ray changes upon the atomic number, it is necessary to assume that this figure depends upon the algebraic sum of the negative and positive charges in the two inner regions of the atomic structure. The proper atomic numbers may be arrived at in several ways; but for the present it will be sufficient to indicate two of these. In the first place, it may be assumed that all the members of a Series in the Periodic System have the same negative charge in their cores; whilst the number of charges in the positive zone increases by single units as we pass up the series from element to element. A second arrangement giving the same atomic numbers would result if the core charges increase by single units from element to element; whilst the corresponding positive charges increase in steps of two units. The charges in the "cometary" orbits will remain constant throughout the series if it be taken for granted that each unit increase in valency corresponds to the possibility of inserting an electron into the "cometary" orbit, an assumption

* See p. 208. 
which corresponds to Ramsay's conception * of the electron as an element. ${ }^{1}$

The following figures (which are intended to be merely illustrative, though they may possibly correspond to reality), give an idea of the results obtained under the two assumptions given above :-

Case I.

\begin{tabular}{|c|c|c|c|c|c|c|c|c|}
\hline & & Li. & Be. & B. & C. & N. & 0. & F. \\
\hline $\begin{array}{c}\text { Electrons in } \\
", ", \\
", "\end{array}$ & $\begin{array}{l}\text { core } \\
\text { positive zone } \\
\text { "cometary" orbits }\end{array}$ & $\begin{array}{l}2 \\
5 \\
2\end{array}$ & $\begin{array}{l}2 \\
6 \\
2\end{array}$ & $\begin{array}{l}2 \\
7 \\
2\end{array}$ & $\begin{array}{l}2 \\
8 \\
2\end{array}$ & $\begin{array}{l}2 \\
9 \\
2\end{array}$ & $\begin{array}{r}2 \\
10 \\
2\end{array}$ & $\begin{array}{r}2 \\
11 \\
2\end{array}$ \\
\hline \multicolumn{9}{|c|}{ Case II. } \\
\hline $\begin{array}{c}\text { Electrons in } \\
", \quad " \\
",\end{array}$ & $\begin{array}{l}\text { core } \\
\text { positive zone } \\
\text { "cometary" orbits }\end{array}$ & $\begin{array}{l}1 \\
4 \\
2\end{array}$ & $\begin{array}{l}2 \\
6 \\
2\end{array}$ & $\begin{array}{l}3 \\
8 \\
2\end{array}$ & $\begin{array}{r}4 \\
10 \\
2\end{array}$ & $\begin{array}{r}5 \\
12 \\
2\end{array}$ & $\begin{array}{r}6 \\
14 \\
2\end{array}$ & $\begin{array}{r}7 \\
16 \\
2\end{array}$ \\
\hline
\end{tabular}

Now, for the sake of simplicity, we may assume that the atoms of the ordinary elements which find places in the Periodic Table are constructed on the model of Case II.; and it may be suggested that at lanthanum a change in atomic architecture takes place, so that the rare earth elements are constructed on the system indicated in Case I. When tantalum is reached, the structure of the atom reverts to the original type of Case II. and continues in this form up to uranium. $\dagger$

A further point of interest arises here. All the rare earth elements are trivalent $\ddagger$; and this implies that as the positive charge in the atoms increases with the rise of the atomic number there must be a corresponding increase in the number of negative electrons in the "cometary" orbits in order to reestablish the proper valency, since the valency of the atom is assumed to be the algebraic sum of the total negative and

* Ramsay regarded hydrogen as " hydrogen electride," metallic magnesium as "magnesium di-electride," metallic cobalt as "cobalt tri-electride," and so on.

† Or possibly the A and B sub-groups in the Periodic Table are composed of differently constructed elements, whilst the rare earth group owes its peculiar character to the additional factor dealt with in the next paragraph.

$\ddagger$ Even cerium shows the trivalent character.

1 Ramsay, Trans., 1908, 93, 774. 
positive charges in the system as a whole. This conception of the rare earth group, therefore, leads to the conclusion that the "cometary" orbits of the rare earths contain an extra set of electrons. From this, two results would follow. In the first place, the influence of these extra electrons in the "cometary" orbits would produce a "blurring" of properties throughout the group of elements, as was made clear above; and, in the second place, since these "cometary" electrons appear to be closely connected with absorption spectra (as can be seen from the cases of ferrous and ferric iron) it might be expected that the absorption spectra of the rare earths would display an unusual complexity - in which again theory and practice accord with one another.

With regard to isobares and pseudo-isobares Stewart's model atom furnishes a simple solution of the problem. When the change from one isobare to another involves the alteration of the atomic number, the electrons are ejected from the core of the atom and their loss permanently influences the numerical value of the surplus positive charge in the two inner regions. On the other hand, when pseudo-isobarism arises from a change in the "cometary" zone (as in the case of simple change of valency by an atom) then the effect upon the two inner zones is only temporary and periodic; with the result that there is a change in general chemical character but no alteration in atomic number. It might simplify the matter if such isobares were regarded as "pseudo-elements" whilst the isobares arising from alterations in the core were treated as "true" elements.

Finally, Stewart's atom suggests a possible explanation of the instability of the radio-elements. The radius of the positive zone is not great when compared with the diameter of the negative electron; and if the number of the latter electrons in the core be increased beyond a certain point there will be insufficient space for them to move freely within the positive zone. ${ }^{1}$ When the atom reaches this degree of complexity, two things may happen to relieve the strain: either negative electrons may be forced out of the atom in the form of $\beta$-rays; or some of the negative electrons may be drawn into the positive zone to form the "sun-and-planet" arrangements

1 Compare J. Q. Stewart, Science, 1917, 46, 568. 
which were postulated in order to account for the $a$-particles. Thus radioactivity could only be expected as a general rule among the elements of complex atomic structure and high atomic weight.*

\section{The Problem of Atomic Weight.}

An obvious rough relationship exists between atomic number and atomic weight, since the latter is approximately double the former figure. Closer examination reveals that the ratio between the two values is not constant. Thus calcium has the atomic weight 40 and an atomic number 20 , so that here the ratio is $2: 1$. Strontium has the atomic weight 87.6 and the atomic number 38 , so that in this case the ratio is $2 \cdot 31: 1$. In barium it rises to $2.45: 1$. Radium yields $2.57: 1$; and finally; in the case of uranium, the value is $2.59: 1$.

Now apparently the atomic number corresponds to the surplus positive charge in the interior of the atom; so that the foregoing figures really imply that the ratio of mass to surplus positive charge increases steadily as the series of elementis is ascended.

This can be explained quite easily if it be assumed that the mass of the atom is purely electrical. On this view, the atomic weight is simply the sum of the electrical masses of the various charges within the atomic structure. Now the mass of an electrical charge can be made to increase if the velocity with which the charge is moving be accelerated to near the velocity of light. If it be imagined that the positive charges in the calcium atom are revolving comparatively slowly in their orbits and that each charge corresponds to two units of atomic weight, then the charges in the radium atom, if they also were moving slowly, would produce an atomic weight of $88 \times 2=176$. Actually, however, the atomic weight of radium is 226 , and to account for this it is necessary to assume that the charges within the radium atom are revolving much more rapidly than those within the calcium atom. The approximate speed required to produce this change in mass is given by the equation-

* It must be admitted that the case of potassium requires some further explanation. 


$$
\frac{m}{m_{0}}=\sqrt{\frac{1}{1-\frac{v^{2}}{c^{2}}}}
$$

where $m_{0}$ is the original mass of the charge at low speeds; $m$ is the mass at velocity $v$; and $c$ is the velocity of light: and, on working this out, the required velocity is found to be 0.6 of the speed of light. The actual velocities of $\beta$-particles ejected from radium have been experimentally found to be 0.52 and 0.65 of the velocity of light; which shows that certain intra-atomic particles do attain velocities of this order of magnitude.

This view of atomic mass can be used to furnish a solution of the isotope question. If it be assumed that in two isotopic elements those parts of the atom which furnish the mass are identical in the two cases but that in each case the intra-atomic machine is revolving at a different speed, then obviously the masses of the two atoms would be different. At the same time, owing to their identity in construction, they would have identical chemical properties.

Further consideration suggests that possibly this "velocityfactor" lies at the root of the Geiger-Nuttall relation, since that relation obviously rests upon a physical rather than upon a chemical basis. 


\section{CHAPTER XIX}

THE PERIODIC LAW

\section{Mendeléeff, Lothar Meyer and Crookes.}

IN its outlines, the history of our views on the elements is the narrative of a conflict between two opposed conceptions: continuity and discontinuity. The attempts of the alchemists to convert lead into gold prove that the idea of continuity had taken root in their minds, and that they did not regard the metals as totally unrelated materials. With Boyle, discontinuity found its first scientific expression in the definition of elementary forms of matter; but early in last century Prout revived the hypothesis of continuity once more by assuming that all the elements were built up from hydrogen. Again discontinuity appeared during the period in which the exact determination of atomic weights was undertaken; and for many years it seemed to be conclusively established that no theory of a homogeneous matter could be produced which would account for the known facts. Yet in 1888, Crookes again revived the discarded hypothesis, though without diverting the main trend of chemical opinion in its favour. At last the phenomena of radioactivity turned the scale; for at the present time continuity appears to be regarded as the final solution of the problem, and electricity has taken the place of Prout's hydrogen and Crookes' protyle. How long this phase will last, it is impossible to prophesy ; but judging from history, it is unsafe to assert that even now either of the conceptions has reached its final stage; and it seems probable that fifty years hence a new set of phenomena may have been discovered which will bring discontinuity again into favour.*

* Even at the present day we have the conception of electricity including positive and negative varieties, which shows the idea of discontinuity again. 
Bearing the foregoing facts in mind, it is not without interest to turn back to the early history of the Periodic Law and to examine the ideas which guided Mendeléeff and Lothar Meyer in their development of the subject; for, curiously enough, their views diverged markedly from each other although their results were in harmony.

If one may judge from his writings, Mendeléeff inclined by preference to numerical data; and since numbers are always discontinuous, he was led, possibly unconsciously, to the conception of the elements as distinct forms of matter isolated entirely from each other even though displaying certain general resemblances. As he himself explains, ${ }^{1}$ he selected as the basis of his speculations the atomic weights and the compoundforming capacities of the elements, and he deliberately excluded from his survey the physical properties of matter (such as cohesion, specific gravity, etc.), on the ground that exact numerical data with regard to them were lacking at that time. In other words, he chose atomic weight and valency as his criteria; and since the law of multiple proportions holds good, he was naturally inclined to regard the atoms of each element as uniform in properties and entirely different from those of any other primary material. He objected strongly ${ }^{2}$ to the employment of graphic methods of expressing the Periodic Law, on the ground that such methods did not indicate the existence of a limited and definite number of elements in each period.

Lothar Meyer, ${ }^{3}$ on the other hand, proceeded on the assumption that atoms are aggregates formed from one and the same type of matter and differ from each other only in their masses. For the purpose of tracing the change in properties produced by increase in mass, he selected the atomic volume as his index. He thus started from a narrower basis than Mendeléeff did; but the results obtained by him were much wider in scope than those arrived at by the tabular method. The difference in the two lines of thought is most easily perceived if it be borne in mind that Mendeléeff's investigations led directly to the calculation of accurate values

1 Mendeléeff, Annalen (Suppl.), 1872, 8, 134.

2 Mendeléeff, Principles of Chemistry (1897), II., $19 \mathrm{ff}$.

${ }^{3}$ Lothar Meyer, Annalen (Suppl.), 1870, 7, 358. 
for the properties of unknown elements, whilst Lothar Meyer's generalization brought into prominence a large number of physical resemblances between the elements which, up to that time, had not been seen as a whole.

To the acute and far-ranging mind of Crookes we owe the conception which enables us to fuse together the apparently irreconcileable ideas of continuity and discontinuity. In his Presidential Address to the Chemical Society in 1888, he was led to speculate ${ }^{1}$ upon the ultimate nature of matter; and he drew attention to the difficulties which would be involved if some forms of matter proved to be unclassifiable within the Periodic System. "If we suppose the elements reinforced by a vast number of bodies slightly differing from each other in their properties, and forming, if I may use the expression, aggregations of nebulæ where we formerly saw, or believed - we saw, separate stars, the periodic arrangement can no longer be definitely grasped. No longer, that. is, if we retain our usual conception of an element. Let us then modify this conception. For 'element' read 'elementary group,' such elementary groups taking the place of the old elements in the periodic scheme-and the difficulty falls away."

For almost twenty years Crookes' views received little more than contempt from the average chemist; but with the discovery of the isotopes, Crookes' conception of the meta-elements was found to be justified. What we term "lead" is an "elementary group" the atoms of which differ from one another in the most fundamental property of all, viz., mass.

With the coming of this fresh knowledge, the old clean-cut separation of element from element on a pure atomic weight basis has passed away. Chemical properties and X-ray spectra are now our chief instruments in classification, and it seems possible that soon we must either increase the delicacy of our chemical methods of separation or abandon the chemical criteria as ultimate tests. The chemical properties of matter are so ill-defined that we can hardly regard them as the last word on the subject. The number of molecules contained in even the most microscopic quantity of a reagent is so enormous, that our results are the merest generalizations and tell us very little of the qualities of the individual atoms with which we

1 Crookes, Trans., 1888, 53, 490. 
are dealing. Possibly at some future time our analytical methods will become more refined-positive ray analysis foreshadows something of the sort.

\section{The Periodic Table and the Atomic Volume Curve.}

The Periodic Table, as laid down by Mendeléeff in his writings, exhibits a symmetry which was one of its greatest assets. For some psychological reason, symmetry has an attraction for the human mind; and we are always apt to prefer a regular arrangement to one in which irregularities predominate. Psychological peculiarities are, however, undesirable guides in the search for truth; and a careful examination of the Table in the light of our present knowledge will suffice to show that it can boast of no such symmetry as we are led to expect from the text-books of our student days.

For example, owing to the omission of some of the rare earth elements and by the insertion of blanks, the Table in its original form attained a very high degree of regularity; but since there are, as we know from the X-ray spectra results, only sixteen elements to fill the eighteen vacant spaces in the Table, it is evident that the symmetry of Mendeléeff's system is purely factitious.

Again, the "typical elements" of the second series do not resemble the elements of the fourth series nearly so closely as they approach the odd series elements. For example, carbon, nitrogen, oxygen, and fluorine, all form alkyl derivatives and are thus parallel in their behaviour to germanium, arsenic, sulphur, and chlorine; but no alkyl derivatives of titanium, vanadium, chromium or manganese have been prepared.

Further, in order to produce the appearance of symmetry, Mendeléeff was forced to place copper, silver, and gold in the first group, although there is no known oxide $\mathrm{Au}_{2} \mathrm{O}$ and the stable chloride of gold is $\mathrm{AuCl}_{3}$.

Finally, while the elements of Group VIII. are placed together on the assumption that their highest oxides are of the type $\mathrm{RO}_{4}$, in actual practice only two, osmium and ruthenium, give such compounds, the other seven elements not yielding anything higher than $\mathrm{RO}_{3}$.

These examples are well-known, and are mentioned here 
only for the purpose of enforcing the statement that the symmetry of Mendeléeff's system cannot be sustained at the present day. Fascinating though its cut-and-dried regularity may be, we cannot afford to let symmetry dominate our minds when in actual fact there is no symmetry to be found.

The most superficial examination shows that, instead of being a symmetrical whole, the Table is really pieced together from a series of discrete sections. In the first place we have the two short Periods, beginning with helium and ending with chlorine. These form a clearly-cut section by themselves; for there is nothing to disturb the regular periodicity of the arrangement. Then come the first two long Periods, which also form a complete section in themselves, beginning with argon and ending with iodine. The long Periods do not repeat the exact sequence of the short Periods: for the difference between the $\mathrm{A}$ and $\mathrm{B}$ Groups makes its appearance, and the elements of Group VIII. break the parallelism between the long and short periods. The third long Period, beginning with xenon, marks the complete collapse of the symmetrical arrangement. Xenon, cæsium, and barium follow the usual order; and then come the rare earths and confusion. Thereafter, the usual sequence appears once more at tantalum and continues, probably, down to the missing halogen with the atomic number 85 . With the fourth long Period we reach the radio-elements and enter a new field in which radioactive properties make their appearance.

From the above, it will be seen that the Table contains the following fragments: (1) two short Periods; (2) two long Periods; (3) a broken long Period; (4) the rare earth group; and (5) the radio-elements. The task of fitting together these five portions into a symmetrical whole has hitherto baffled those who have attempted it; but there seems to be no reason for assuming that the problem is insoluble.

Soddy ${ }^{1}$ prefers to draw a distinction between two portions of the Table. The three series of elements, vanadium-germanium, columbium-tin, and tantalum-lead are regarded. by him as "interpolated" elements; and the table is thus divided into two short periods; two long periods each with a hiatus between Groups IV. and V.; an interrupted long period 
beginning with xenon; another long period commencing with niton, the rare earth elements; and three series of "interpolated" elements.

Many other attempts 1 have been made to produce new tabular forms, but none of them combine symmetry with the accurate representation of the elemental relationships.

Leaving the tabular arrangement, we may now proceed to the consideration of the curve of atomic volumes. In the past, it has been usual to plot the abscissæ as atomic weights; but in view of the fact that atomic weights are no longer to be considered as physical constants, it seems desirable to replot the graph, using the atomic numbers on the horizontal axis. The diagram given at the end of this volume shows the result; and it will be seen that the change leads to an even distribution of the points on the curve.

The most casual examination of the figure shows that the curve is not a regular one. There is a steady increase in atomic volume as we pass from lithium, through sodium, potassium, and rubidium to crsium at the maxima of the graph; but if the minima are investigated it will be found that no such regularity exists in that section.* The five known minima, instead of lying in a crescendo or diminuendo order, apparently fall at random, as the following figures show :-

Minimum Element. Carbon. Aluminium. Nickel. Ruthenium. Osmium.

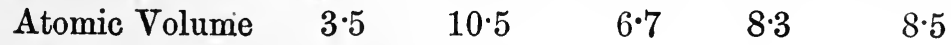

Thus the minima do not occur, like the maxima, at elements analogous to each other in chemical nature; nor do they fall into any clear order in respect to the actual volume of the minimum element in each cycle.

1 See Garrett, The Periodic Law (1909), for a summary of work up to that date; and also Martin, Researches on the Affinities of the Elements; Adams, J. Amer. Chem. Soc., 1911, 33, 684 ; Hopkins, ibid., 1005; Harkins and Hall, ibid., 1916, 38, 169 (cf. Soddy, Chem. Soc. Ann. Report, 1916, 13, 254); Hackh, ibid., 1918, 40, 1023; Steinmetz, ibid., 733; Stackelberg, Zeitsch. physikal. Chem., 1911, 77, 79; Vogel, Zeitsch. anorgan. Chem., 1918, 102,177; Schmidt., ibid., 1918, 103, 79; Loring, Studies in Valency, p. 28; Thornton, Phil. Mag., 1917, 34, 70 (cf. Richards, Trans., 1911, 99, 1201); Haughton, Chem. News, 1888, 58, 93, 102.

* If the curve be plotted with atomic radii as ordinates instead of atomic volumes, it assumes a less irregular aspect, but this is so merely on account of the disparities being reduced by the method of plotting. 
In this connection, it must be borne in mind that our methods of determining the atomic volume of an element at the present time are not free from obvious errors. Atomic volume, in the sense in which the term is used in connection with the curve, is simply a figure obtained when the atomic weight of the element is divided by the density of the element in the solid state. It represents merely the ratio of mass to space occupied. But the "space occupied" includes in itself no less than four factors:-(1) the space actually filled by the minute positive and negative charges of the atomic machine; (2) the spaces between these charges within the atom; (3) the spaces between the atoms in the molecule of the element; and (4) the spaces between the molecules of the element in the mass with which we are dealing. It is self-evident that when we compare two different elements, these four factors are not necessarily identical. For example, in the case of a monatomic element like mercury, the third factor cannot be present; whilst the difference in coefficient of thermal expansion between certain elements proves that the fourth factor varies with temperature:

Mendeléeff ${ }^{1}$ believed that when the atomic volume is large, the atoms in the molecule of the element stand comparatively far apart from each other; whereas those elements possessing small atomic volumes have their atoms closely adjacent to each other in the molecular structure; and to this he traced the difference in reactivity between the two elemental types.* The fact that compressibility seems to be a periodic function ${ }^{2}$ suggests that the inter-molecular spaces depend upon the nature of the compressed element. Further, those elements with large atomic volumes are more readily compressible than the ones near the minima of the Curve.

Again, when the elements in the same family are examined, it is found that the linear coefficient of thermal expansion changes from element to element as we pass down the group.

1 Mendeléeff, 'Annalen (Suppl.), 1872, 8, 156.

2 Richards, Zeitsch. physikal. Chem., 1907, 61, 98; Trans., 1911, 99, 1201; Zeitsch. Elektrochemie, 1907, 13, 519.

* Mendeléeff used the analogy of a sponge to represent a reactive element which can easily be penetrated by the atoms of other elements, whereas a non-reactive element was likened by him to a more solid and less permeable material (Principles of Chemistry, II., 33 (1897)). 
Thus magnesium has a coefficient of 27.5 ; zinc has 29 ; cadmium has 31 ; and mercury has 61 ; but on the other hand the order is reversed in the electronegative families; for the coefficient of chlorine is 470 while bromine and iodine have 390 and $83 \cdot 7$ respectively.

From considerations such as these, it is evident that our present methods of estimating atomic volumes are extremely rough; and hence the irregularity of the atomic volume curve is only what might be expected when we take into account the nature of the data employed in framing it.

It seems not improbable that something of importance might be achieved by an attempt to recalculate the atomic volumes of the atoms by the insertion of corrections based on the known data with regard to compressibility and expansion coefficients. A compressibility correction would reduce the atomic volumes of the alkali series and the halogens; while the correction for expansion would, as far as can be seen, tend to equalize the Curve considerably.

In its present form (see the diagram at the end of this volume), the Atomic Volume Curve contains five waves. The first two of these correspond to the two earliest series in the Mendeléeff Table; and each of them includes eight elements. The next two waves are longer, containing eighteen elements each. The extension is due to the appearance in the middle of the wave of a series of elements (titanium to zinc and zirconium to cadmium) which do not exactly reproduce the characters of Mendeléeff's typical elements. The fifth wave is yet more extended, since in it the rare earth elements are interpolated; and it contains thirty-two elements.

With regard to the intercalary sections of the third, fourth, and fifth waves, it is not without interest to observe that the iron, ruthenium, and osmium groups occupy minima on the Curve corresponding to the carbon minimum in the first wave; and that while the element carbon is pre-eminent in its capacity for chain-formation, the "minimum" groups in the later periods are marked by their complex-salt production. It may be suggested that small atomic volume is connected in some way with the firm retention of atoms or groups in a compound.

Turning to the relations between valency and the atomic volume curve, it may be worth our while to examine the 
conditions in the waves beginning with potassium and rubidium. The following figures give the highest valency exhibited in the oxygen compounds or acids :-

$\mathrm{K}$ Ca Se Ti V $\mathrm{Cr}$ Mn $\mathrm{Fe}$ Co $\mathrm{Ni}$ Ca $\mathrm{Zn}$ Ga Ge As Se $\mathrm{Br} \mathrm{Kr}$ $\begin{array}{llllllllllllllllll}1 & 2 & 3 & 4 & 5 & 6 & 7 & 3 & 4 & 2 & 2 & 2 & 3 & 4 & 5 & 6 & (5) & 0\end{array}$ $\mathrm{Rb}$ Sr $\mathrm{Y} \mathrm{Zr} \mathrm{CbMo}-\mathrm{Ru} \mathrm{Rh} \mathrm{Pd} \mathrm{Ag}$ Cd In Sn Sb Te I X $\begin{array}{llllllllllllllllll}1 & 2 & 3 & 4 & 5 & 6 & ? & 8 & 6 & 4 & 1 & 2 & 3 & 4 & 5 & 6 & 7 & 0\end{array}$

An examination of the above will show that if the abnormality of bromine be neglected, the first series falls into three distinct groups. From potassium to manganese there is a regular increase in valency; from zinc to bromine, if the salts of perbromic acid can be proved to exist, there is again a regular increase in valency; whilst in the intermediate group, from iron to copper, there is no regularity traceable. In the second series, there is a regular increase in valency from rubidium to ruthenium and also from silver to iodine ; but again there is an intercalated group-rhodium and palladium-which diverges from the regular sequence. It is evident that the Atomic Volume Curvé, like Mendeléeff's Table, fails to bring out any perfect symmetry where valency relations are concerned.

With the second type of intercalary elements, the rare earth group, it will be convenient to deal in a separate section of this chapter.

The peculiar resemblances in certain properties between some elements which lie adjacent to one another in the atomic order, find a better expression in the Curve than in the Table. For example, in the case of boron and carbon we have the hydrides $\mathrm{B}_{2} \mathrm{H}_{6}$ and $\mathrm{C}_{2} \mathrm{H}_{6}$, where the similarity in composition is reinforced by the fact that both substances are colourless gases. The close approximation of the two elements on the curve throws up their relationship very much better than does the ordinary tabular arrangement, especially in cases such as this where the valency of boron must actually be "abnormal" in order to produce the similarity.

When we turn to physical properties, however, the supreme advantage of the Curve over the Table becomes manifest. By the superposition of the odd and even series in the Table, the eiements are juxtaposed in a manner which conceals almost completely the general periodicity of physical properties; for, 
intermingled with the soft white metals of the alkali group we get the coloured metals, copper and gold; and among the nonmetallic halogens we find the definitely metallic manganese. In the Curve, each set of physical properties recurs at regular intervals, as can be seen from an inspection of the diagram at the end of this volume.

Lothar Meyer ${ }^{1}$ drew attention to four peculiarities in the distribution of the elements on the Curve. In the first place, elements with small atomic volumes do not appear to possess an electrochemical character which is either strongly positive or strongly negative: carbon, for instance, combines with both hydrogen and chlorine to produce stable derivatives, while iron forms ordinary salts and gives rise to the ferrates as well. In the second place, rapid changes in the slope of the Curve go hand in hand with great alterations in the chemical character from element to element, while a slight change in the inclination of the Curve is paralleled by small differences in chemical nature between two adjacent elements. Thirdly, electropositive elements in the first two waves occur at the maxima or on the downward slopes beyond the maxima, while the electronegative elements are found either at the minima or on the upgrade between a minimum point and the next maximum. In the case of the three longer waves, both maxima and minima are occupied by electropositive elements; the elements adjacent to them are also positive, and electronegative (or else amphoteric) elements take up positions intermediate between the maxima and minima. For example, in the first long wave, potassium occupies the maximum and calcium is the next element on the down-slope; then come titanium, vanadium, and chromium, which, though metallic, give acidic oxides; at the minimum lie iron, cobalt, and nickel, which are metallic in the main; then follow the elements copper, zinc, gallium and germanium, which, though amphoteric in some circumstances, are mainly electropositive; and, finally, on the rising gradient lie the elements arsenic, selenium and bromine, which show increasingly electronegative character as we pass up the series. Lothar Meyer's fourth comment is concerned with the fact that in certain cases two elements may have

1 Lothar Meyer, Modern Theories of Chemistry, 1888, p. 149; Annalen (S uppl.), 1870, 7, 354. 
almost exactly the same atomic volume and yet may differ markedly in chemical properties, as is the case with sodium and chlorine or sulphur and indium.

It seems a matter of interest to trace, if possible, some connection between these observations of Lothar Meyer, and the modern electrical theory of matter, in order to see whether our newer views throw any light upon the points in question. To do that, it is necessary to assume that the "atomic volume" expressed in the curve really gives us an approximate measure of the relative spaces occupied by the atoms themselves; and, as has been noted, this is not necessarily anything but a very rough approximation. Let it be assumed, however, that the "atomic volume" is a measure of the space actually occupied by an atom. If so, the diameter of the outermost electronic orbits must be proportional to the atomic volumes. We may further assume that electropositive character implies a readiness of the system to lose electrons, whilst electronegative character corresponds to an attraction for extra negative electrons towards the system.

Now it is possible to imagine a system in which the dimensions are such that the attraction of the central nucleus, being exerted across a large radius, is not sufficient to attract external electrons through the negative ring of the atom because of the repulsive influence of the ring-electrons which are acting at a comparatively short distance from the external negative electron; and, further, that the attraction of the positive nucleus is sufficient to prevent any of the ring-electrons leaving the atom, because no other positive nucleus can approach close enough to exert an overpowering attraction on the ring-electrons. Such a case would represent the inert gas atoms.

If the atomic radius be increased beyond this critical value, then the attraction of the positive nucleus will be correspondingly weakened; and electrons will be more easily enabled to quit the atom under external attractions. This case would correspond to the alkali metals.

If the critical radius be reduced, the pull of the central nucleus on the ring-electrons will be strengthened; and hence they will show no tendency to leave the system: while at the same time, owing to the possibility of near approach of external electrons being increased, it becomes more possible for electrons 
to be drawn into the atom from other systems. This case, then, corresponds to the halogen atoms.

The results of still further contraction of the orbital radius will be governed by two opposed factors. In the first place, the smaller the radius, the greater the extension of the influence of the positive nucleus as compared with the diameter of the ring; while in the second place the ring-electrons will be more closely adjacent in space in the contracted circuit and they will therefore tend to repel each other and so facilitate their withdrawal from the atomic system. It seems probable that the alternation of positive and negative characters in the elements in the lower parts of the wave is due to the ascendancy attained by each of the factors in turn.

There remains the problem of accounting for the fact that elements such as sodium and chlorine differ entirely in properties whilst their atomic volumes are identical. Sodium has a positive nucleus of eleven charges while the positive nucleus of chlorine contains seventeen charges. Since the orbital radii are equal in the two cases, it is evident that the ring-electrons of sodium will be much more weakly attracted than is the case in chlorine; and also that the tractatory power of the chlorine nucleus upon negative electrons just outside the ring will be greater than that of the sodium nucleus. The counter-acting factor-repulsive influence of the ring-electrons upon external negative charges-is, of course, also increased in the case of chlorine; but since the positive charges are grouped together in a small space whereas the negative charges are extended over a large circumference, it seems reasonable to assume that the repulsive effect (as far as purely local results are concerned) will only be slightly increased as compared with the increase in the pull of the positive nucleus.

There is one factor which is not well expressed in the periodic curves as hitherto drawn. Some concrete examples will serve to make the point clear. In passing from lithium, with the atomic number 3 , to beryllium, with atomic number 4 , it is clear that the addition of a single positive charge to the nucleus represents an actual increase of the charge by onethird: whereas the addition of a positive charge to the zinc atom only results in a change of one-thirtieth of the original charge, since the atomic number of zinc is 30 . When the 
curve is plotted either with atomic weights or atomic numbers as abscissæ, this disparity in the relative values of the additional charge is not brought out; and it seems of interest to discover some graphic method which will put the matter in a clearer light.

If the abscissæ be represented by the logarithms of the atomic numbers, the desired result is attained, as then equal changes in the abscissæ represent equal fractional differences in the nuclear charge, no matter at what point on the curve they may be measured. Further, if the ordinates be made proportional to the logarithms of the atomic volumes, any multiple relationship between the two factors will be expressed in the graph.

The result is to be seen in Fig. 23. Examination of the curve will show that the wave beginning with lithium is much broadened as compared with that on the ordinary type of graph, while the succeeding waves are made more and more narrow as we pass along the line. The meaning of this will be apparent from a numerical example. Beryllium lies at the point 0.60 while boron is situated at 0.70 , leaving a gap of 0.10 between them; magnesium is placed at 1.08 and aluminium at $1 \cdot 11$, the gap in this case being only $0 \cdot 03$. The chemical differences between beryllium and boron are too obvious to need recalling to the reader; whereas magnesium and aluminium are much more closely alike. Evidently, on this system of plotting, differences in the abscissæ correspond to some extent to differences in chemical nature: ${ }^{*}$ the greater the differences between the abscissæ of two adjacent elements, the greater the chemical differences between them. In the light of this, the compression of the succeeding waves becomes explicable, for it is well known that with increase in atomic number even the electronegative halogens become endowed with semi-metallic character and resemble the rest of the elements more closely. Again, the differences in chemical and physical properties between iron, cobalt, and nickel on the one hand, the ruthenium and osmium groups on the other is illustrated by the broadened form of the curve at the iron triad as compared with the sharper points of inflexion at ruthenium and osmium.

* This is merely given for the sake of easy reference, since actually the important factor would appear to be the length measured along the curve from element to element. 


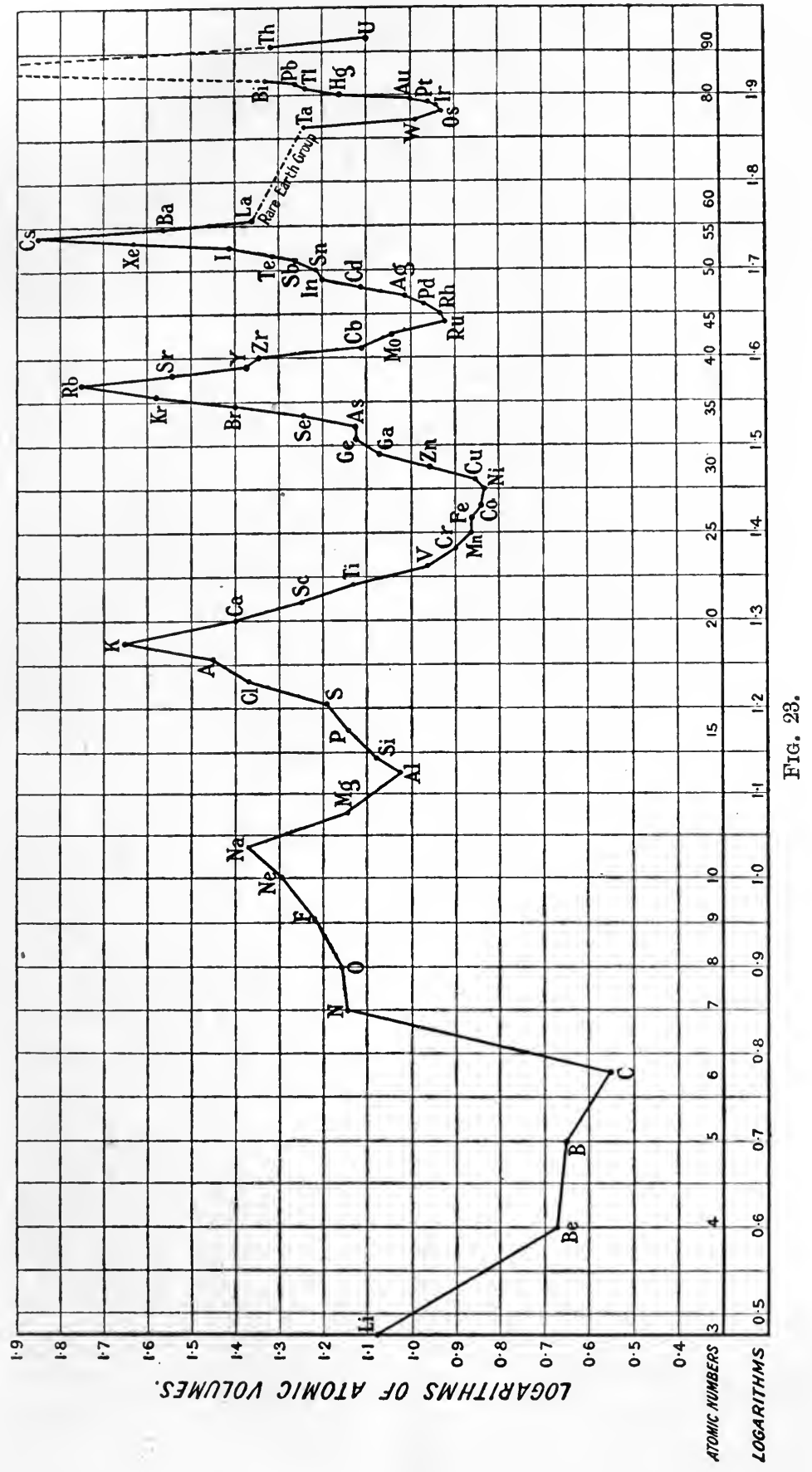




\section{Spiral Arrangements of the Elements.}

The first attempt to arrange all the elements in a periodic grouping took the form of a three-dimensional model-the Telluric Helix of de Chancourtois; ${ }^{1}$ and it is not surprising that from time to time attempts have been made to utilize the third dimension as an aid to classification. It cannot be said that much light has been thrown on the matter by these essays; but some account of them must be given here for the sake of completeness.

Reynolds ${ }^{2}$ put forward the analogy of a vibrating knotted string to represent the periodic arrangement; and his proposal was modified by Crookes ${ }^{3}$ a couple of years later. Crookes suggested that the Atomic Volume Curve represented the projection of a spiral figure which might either be a simple spiral or one of the "figure-of-eight" type. The elements of low atomic weight were grouped in the upper turns of the spiral and the valency was represented by dividing the separate sections of the curve into sectors each of which contained an element, so that the members of the same family stood above one another on the spiral. In Crookes' view, his spiral represented the effect of the interplay of two forces-electrical charge and temperature. Movement along the spiral, if projected on a horizontal plane, indicated the change in electrical character; while the vertical fall occasioned by the descent of the point in travelling along the spiral represented a lowering of temperature.

A somewhat similar model was proposed by Harkins and Hall. ${ }^{4}$ It is differentiated from that of Crookes by the fact that the rare earth elements are all placed in a vertical line instead of being grouped round the spiral. Stewart 5 suggested a three-dimensional model on the principle of bringing into line all elements showing the same numerical valency and

1 See Compt. rend., 1891, 112, 77 ; and also Nature, 1889, 41, 186, for an account of de Chancourtois' work which was first published in 1862.

2 Reynolds, Chem. News, 1886, 54, 1 ; Nature, 1895, 51, 486.

3 Crookes, Chem. News, 1886, 54, 115; Trans., 1888, 53, 487.

4 Harkins and Hall, J. Amer. Chem. Soc., 1916, 38, 169.

5 Stewart, Recent Advances in Physical and Inorganic Chemistry, 3rd Edition, 1918. 
differentiating between the three different types of transition which occur at Group VIII., Group IV., and the Zero Group. Soddy ${ }^{1}$ proposed a spiral which combines to some extent the normal and figure-of-eight curves in a single model.

A plain spiral arrangement was put forward by Stoney, ${ }^{2}$ who showed that the atomic weight relationships of the elements could be depicted by means of a logarithmic spiral ; but owing to the modern disrepute into which atomic weights have fallen, it may be doubted if this plan meets the case.

The main drawback to the spiral representation appears to be that in it no new facts are brought to light, and there is no fresh collocation of the allied elements which might give it an advantage over the ordinary forms of classification. Also, in most cases it is more difficult to grasp as a whole. Soddy's arrangement seems the most perspicuous, if the spiral grouping be adopted.

\section{The Periodic Surface.}

The Periodic System is a pure matter of classification; for we have no inkling of the factors which produce the recurrent resemblances among the elements. Prolonged study of the Table always leaves on the mind the impression that behind this curious grouping there is some simple law which, when it is at last discovered, will seem almost self-evident: but up to the present nothing has been suggested which throws any real light upon the subject. Obviously our immediate effort should be directed to producing an arrangement which, regardless of pre-conceived ideas, will bring out as far as possible all the known similarities; for when such a system is evolved there is a certain chance that it may suggest to our minds the esoteric influences which control the periodicity of properties among the various forms of matter with which we are acquainted.

In the foregoing pages it has been shown that Mendeléeff's Table and Lothar Meyer's Curve each possess valuable individual characteristics. The Table shows at a glance the

1 Soddy, Chemistry of the Radio-elements, II., 9, 1914.

2 Stoney, Phil. Mag., 1902, 4, 411, 504 ; see also Rayleigh, Pro. Roy. Soc., 1911, A, 85, 471; and compare Harkins and Hall, J. Amer. Chem. Soc., 1916, 38, 189; Loew, Zeitsch. physikal. Chem., 1897, 23, 3 ; Stintzing, ibid., 1916, 91, 500 ; Caruelley, Chem. News., 1886, 53, 183. 
general chemical relationships of the elements; but without undue complication of lettering, it is impossible in it to bring out simultaneously the corresponding periodicity in physical properties. The Curve, on the other hand, exhibits the recurrence of physical properties; but by its very nature it fails to demonstrate clearly the relations between the elements of a given family, since the members are scattered over the various waves and not collected together. Obviously it would be advantageous if the good points of both representations could be combined in a single system, exhibiting simultaneously chemical and physical properties.

Before this can be done, however, it is necessary to rearrange the Mendeléeff Table; for, as it stands, the alternation of the A and B sub-groups would throw the physical properties into a disorderly series. The following arrangement was adopted to produce the model illustrated in the frontispiece to this volume.

$\mathrm{He} \mathrm{Li} \mathrm{Be}$

$\begin{array}{lllllllllllllll} & \mathrm{C} & \mathrm{C} & \mathrm{N} & \mathrm{O} & \mathrm{F}\end{array}$

$\mathrm{Ne} \mathrm{Na} \mathrm{Mg} \mathrm{Al}$

Si $\mathrm{P} \quad \mathrm{S} \quad \mathrm{Cl}$

A K Ca Sc Ti V Cr Mn Fe Co Ni Cu Zn Ga Ge As Se Br $\mathrm{Kr} \mathrm{Rb} \mathrm{Sr} \mathrm{Y} \mathrm{Zr} \mathrm{Cb}$ Mo - Ru Rh Pd Ag Cd In Sn Sb Te I $\mathrm{Xe} \mathrm{Cs} \mathrm{Ba}$ Rare Earth Elements

$\mathrm{Nt}-\mathrm{Ra}$ Ac Th $\mathrm{UX}_{2} \mathrm{U}$

$\mathrm{Ta} \mathrm{W}-\mathrm{Os}$ Ir Pt $\mathrm{Au} \mathrm{Hg} \mathrm{Tl} \mathrm{Pb} \mathrm{Bi} \mathrm{Po-}$

As can be seen, the two short periods have been broken up and the elements in them have been distributed as far as possible along with their nearest analogues in the long periods. Thus fluorine is classed together with the halogens, oxygen and nitrogen along with sulphur and phosphorus. Carbon has been placed with silicon rather than with titanium and its analogues. The positions of beryllium, magnesium, and aluminium have been selected on account of the metallic character of the elements ; but it must be admitted that they might with equal justice have been transferred to the corresponding positions over zinc and gallium.

If now, from each point representing an element in the above scheme a perpendicular be raised with a length proportional to the atomic volume of the element and if the summits of these perpendiculars be united by a surface, the 
three-dimensional model shown in the frontispiece is produced.*

The advantages which this form of representation possesses over the other two are obvious on inspection (see frontispiece). In the first place, by getting rid of the alternation of $\mathrm{A}$ and $\mathrm{B}$ sub-groups, it brings into direct contiguity the "atomic analogues" of Mendeléeff and separates from one another the oxygenic and basigenic elements which are associated in the Mendeléeff Table. Secondly, it throws into prominence the abnormality of the rare earth group, as can be seen from the abrupt elevation in the centre of the model, which is quite out of harmony with the rest of the contours. Further, it will be found that all elements giving coloured chlorides in their highest stage of valency fall compactly together in the centre of the model. Finally, while the Atomic Volume Curve only shows the change of slope in passing from one element to the next in the atomic order, the Periodic Surface exhibits simultaneously the volume relations of neighbouring elements and also those existing between members of the same family.

It is frankly to be admitted that the model possesses very little of the symmetry to which we have been accustomed; but as has already been pointed out, if we have to choose between factitious symmetry and actual correlation of facts, we must decide in favour of the latter, discomforting though the choice may be.

\section{The Problem of the Rare Earth Elements.}

Of all the problems centering in the Periodic Table, the position of the rare earth elements is the most difficult. From the X-ray spectra, it appears to be established that between lanthanum and tantalum there are fifteen elements in the atomic order; and if celtium be placed next to tantalum, this leaves only one element of the group still to be detected, which will have the atomic number 61 and will lie between neodymium and samarium.

Numerous attempts have been made ${ }^{1}$ to find a satisfactory

* Full directions for the construction of a slightly improved model are given in the Appendix.

1 Thomsen, Zeitsch. anorgan. Chern., 1895, 9, 190 ; Brauner, ibid., 1902 , 32, 1; Rudorf. ibid., 1903, 37, 177 ; Benedicks, ibid., 1904, 39, 41 ; Vogel, 
arrangement of the rare earth elements; but up to the present no generally accepted solution of the problem has been reached. The proposals hitherto put forward may be grouped under three heads: (1) the elements may be placed all together in one group of the Table; (2) they may be made to form a bridge connecting lanthanum with tantalum, outside the ordinary tabular arrangement; and (3) they may be distributed among the groups in a more or less symmetrical manner. These three views may be dealt with in turn.

Owing to the general similarities in the rare earth family, it has been proposed that they should be arranged in a single group of the Table. Since they all form oxides of the type $\mathrm{R}_{2} \mathrm{O}_{3}$, the third group seems the most appropriate; but there is an alternative suggestion by Brauner that lanthanum should be left in Group III. while the elements from cerium to the predecessor of tantalum are placed in Group IV. Brauner's actual arrangement, however, necessitates the existence of no less than twenty elements between lanthanum and tantalum; so it does not agree with the present state of our knowledge. R. Meyer removes cerium to Group IV. and leaves all the rest of the family in Group III. ; but this arrangement deviates too much from the known atomic order. From these examples, it will be seen that any attempt to retain even the majority of the elements in a single group is open to grave objections.

The second suggestion-that the rare earth family has no place in the Table but forms a "bridge" between lanthanum and tantalum-is one which can hardly recommend itself in view of the regular sequence into which the X-ray spectra fall. It has been proposed that the elements should be placed in a horizontal plane while the rest of the Table is inscribed on a vertical surface; but this idea conveys nothing very definite to the ordinary mind. It has also been suggested by Vogel that the normal elements in the Table should be placed on a regular spiral of eight places; while the rare earth group ibid., 1918, 102, 177 ; Retgers, Zeitsch. physikal. Chem., 1895, 16, 651; Baur, ibid; 1911, 76, 569 ; Biltz, Ber., 1902, 35, 562 ; Werner, ibid., 1905, 38, 914 ; Meyer, Physical Zeitsch., 1918, 19, 178 ; Brauner, Zeitsch. Elektrochem., 1908, 14, 525; Hicks, Phil. Mag., 1914, 28, 139 ; Steele, Chem. News, 1901, 84, 285 ; Harkins and,Hall, J. Amer. Chem. Soc., 1916, 38, 169 ; Wyrouboff and Verneuil, Ann. chim. phys., 1906, 6, 466; Reychler, Les théories physico-chimiques, 1903, p. 50; Soddy, Chemistry of the Radio-elements, II., 9, 1904. 
should be accommodated on a subsidiary spiral inserted after barium. Here also there seems to be no very concrete reality behind the representation.

With regard to the distribution of the rare earth group over the ordinary Table, it is impossible to fit the rare earth elements into 'Mendeléeff's arrangement owing to the disparity in numbers between the blanks and the elements predicted by the $\mathrm{X}$-ray spectra. Also, owing to the peculiar character of the rare earth group, it is straining probability too far if we assume that one of these elements can take its place among the halogens. If the rare earth family is to be distributed among the "normal" elements, it must be done by means of some new arrangement.

The following new grouping seems worth considering. Although it has many good points, it is not to be regarded as a final solution, but is put forward mainly in the hope that an examination of it may suggest some more perfect system.

According to Moseley's results, the rare earth elements follow in natural sequence after cæsium and barium; and it is therefore reasonable to look for their congeners in corresponding places in the rest of the Table. Taking valency as our first guide, we find that the rare earth elements, with the exception of cerium, form stable oxides of the type $\mathrm{R}_{2} \mathrm{O}_{3}$; and as a beginning we may search out those elements in the other series which also yield this oxide form. Very little consideration will show that the required elements form definite and limited series, one of which runs from scandium to cobalt,* another from yttrium to rhodium. Now if we follow out this train of analogy, we shall be led to group the rare earth elements in the following manner :-

$\begin{array}{lllllllllll}\mathrm{Rb} & \mathrm{Sr} & \mathrm{Y} & \mathrm{Zr} & \mathrm{Cb} & \mathrm{Mo} & - & \mathrm{Ru} & \mathrm{Rh} & \mathrm{Pd} & \mathrm{Ag} \\ \mathrm{Cs} & \mathrm{Ba} & \mathrm{La} & \mathrm{Ce} & \mathrm{Pr} & \mathrm{Nd} & - & \mathrm{Sm} & \mathrm{Eu} & & \\ & & \text { Gd } & \text { Tb } & \text { Dy } & \text { Ho } & \text { Er } & \text { Tm } & \text { Yb } & & \\ & & \text { Lu } & \text { Ct } & \text { Ta } & \text { W } & - & \text { Os } & \text { Ir } & \text { Pt } & \text { Au }\end{array}$

At this point it may be well to apply the atomic weight test, as a rough clue to the accuracy of the arrangement. Owing

* The oxide $\mathrm{Ni}_{2} \mathrm{O}_{3}$ is apparently a mixture of $\mathrm{NiO}$ and $\mathrm{NiO}_{2}$ and not a true compound; palladium sesquioxide is unstable; and the anhydrous form of $\mathrm{Pt}_{2} \mathrm{O}_{3}$ has not been obtained. 
to the similarity in general character, it may be assumed that the intermediate row of elements beginning with gadolinium should have atomic weights approximately equal to the average of the atomic weights of the elements above and below them. In other words, the atomic weight of gadolinium should lie near the mean of those of lanthanum and lutecium. The following figures show the results, the figures marked "Calculated" being those of the averages:-

$\begin{array}{lcccccc} & \text { Gadolinium. } & \text { Dysprosium. Holmium. } & \text { Erbium. } & \text { Thulium. } & \text { Ytterbium } \\ \text { Experiment } & 157 \cdot 3 & 162.5 & 163.5 & 167 \cdot 7 & 168 \cdot 5 & 173 \cdot 5 \\ \text { Calculated } & 157 \cdot 0 & 161 \cdot 0 & 164 \cdot 1 & 167 \cdot 4^{*} & 170 \cdot 7 & 172.5\end{array}$

It will be seen that the agreement is remarkably good in some cases and is not bad on the whole. On the other hand, the agreement is by no means good when we calculate a mean value for cerium by taking the average of the atomic weights of zirconium and terbium.

Since the rare earth elements form salts of various tints, it seems advisable to see whether the suggested arrangement brings to light any parallelisms in this field. The table below shows the state of the matter, the chlorides being chosen as the simplest salts in which complications are unlikely to present themselves and the highest chloride being selected in each case.

* Calculated from the values for Nd, Sm, W and Os. 


\begin{tabular}{|c|c|c|c|c|}
\hline 息界 & 无思 & & & 嵒思 \\
\hline 马䡒 & 这思 & & & 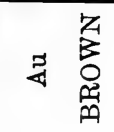 \\
\hline 亖畕 & 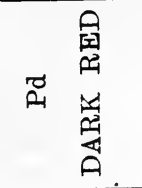 & & & 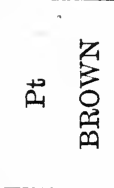 \\
\hline 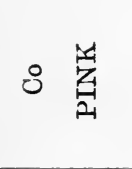 & 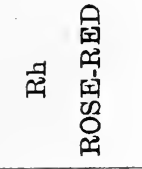 & 呈总 & 䎴思 & 与兽 \\
\hline 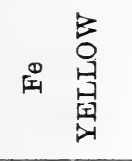 & 品葛 & 昆曷 & 舀畺 & 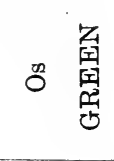 \\
\hline 豆总 & 1 & 1 & 芶营 & 1 \\
\hline 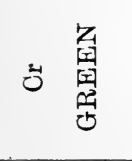 & 总兽 & 芸界㖥 & 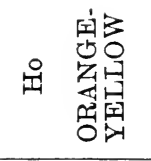 & 》总 \\
\hline 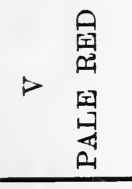 & 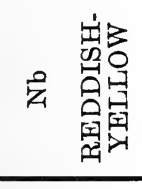 & 盖兽 & 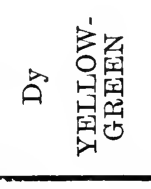 & 总总 \\
\hline 无思 & 岕思 & 。罟 & 思 & 己 \\
\hline 品界 & メ思 & 。思思 & 焉 & 寻界 \\
\hline
\end{tabular}


An examination of the above scheme will reveal certain points of interest. In the first place, as shown by the heavy line, all the elements yielding coloured chlorides lie in a single compact area. Secondly, there is a certain symmetry in the arrangement, since on the right-hand side there is a regular shift to the left of one place as we reach the end of the coloured salts, while on the left-hand edge of the scheme there is also a regularity; for all the elements giving colourless chlorides are grouped there.*

Further examination of the arrangement will show that it contains certain harmonies in the salt-colours which are not without interest. All chlorides of elements in the first column are white. In the second column, cerium is the only exception to the regular whiteness; and there is no doubt whatever as to the place of cerium in the arrangement, since it follows lanthanum in Moseley's sequence. The third column exhibits a regular gradation of tint in praseodymium, dysprosium, and tantalum; and the same may be said of the adjacent grouping : neodymium, holmium, and tungsten. In the next column the pink of the manganese salts is brought into correspondence with the pink of the erbium derivatives. The next column brings into prominence the close colour-alliance of iron, ruthenium, and samarium on the one hand, and thulium and osmium on the other. Finally, europium is placed in a proper colour-relation to cobalt and rhodium.

It must be admitted that this evidence presents features of interest, even if the weight assigned to it is not excessive.

When we come to the oxides, it will be found that the arrangement suggested above brings out another point. Among the rare earth elements, cerium, praseodymium and terbium are marked out by the fact that they furnish oxides of the type $\mathrm{RO}_{2}$. It will be noticed that the system suggested here places both cerium and terbium in the titanium group. Titanium and zirconium yield oxides of the types $\mathrm{R}_{2} \mathrm{O}_{3}$ and $\mathrm{RO}_{2}$, which brings these elements into line with cerium and terbium $\dagger$; and, like

* It may be noted that if cerous chloride had been chosen instead of ceric chloride, then the colour in that case would have been white and the only exception would disappear.

$\dagger$ The impure "peroxide" of terbium appears to correspond to the formula $\mathrm{Tb}_{4} \mathrm{O}_{7}$ and may be a mixture of $\mathrm{Tb}_{2} \mathrm{O}_{3}$ and $\mathrm{TbO}_{2}$. 
cerium also, titanium and zirconium yield the stable dioxide, while their sesquioxides are less stable.

In the next column we find praseodymium classed along with vanadium, columbium, and tantalum. These last three elements yield both types of oxides $\mathrm{R}_{2} \mathrm{O}_{3}$ and $\mathrm{RO}_{2}$; and it is of interest to note that praseodymium gives rise to corresponding compounds $\mathrm{Pr}_{2} \mathrm{O}_{3}$ and $\mathrm{PrO}_{2}$.

Thus the system suggested appears to fit in well with the valency peculiarities of the rare earth elements.

Turning to another line of evidence, the molecular volumes of the rare earth chlorides may be examined. It will be seen from the figures below that there is a steady contraction in volume from lanthanum to samarium; and thereafter a rise, so that gadolinium may be regarded as the beginning of a new series. This agrees with the arrangement suggested.

$\begin{array}{cccccccccc}\mathrm{La} & \mathrm{Ce} & \mathrm{Pr} & \mathrm{Nd} & - & \mathrm{Sm} & \text { (Eu) } & \mathrm{Gd} & \mathrm{Tb} & \mathrm{Dy} \\ 63.8 & 62 \cdot 9 & 60 \cdot 8 & 60 \cdot 6 & ? & 57 \cdot 5 & ? & 58 \cdot 3 & 61 \cdot 1 & 73 \cdot 3\end{array}$

The evidence accumulated above is sufficient to show that the foregoing system deserves a certain amount of consideration. Against it may be urged the fact that it takes no note of the relations which are known to exist between the solubilities of the double salts of the rare earth group, since it splits the terbium group in half. But solubility is hardly a final test in a case such as this; though it must be allowed its due weight as evidence.

The complete Periodic Table drawn up on this basis is printed on the following page. 


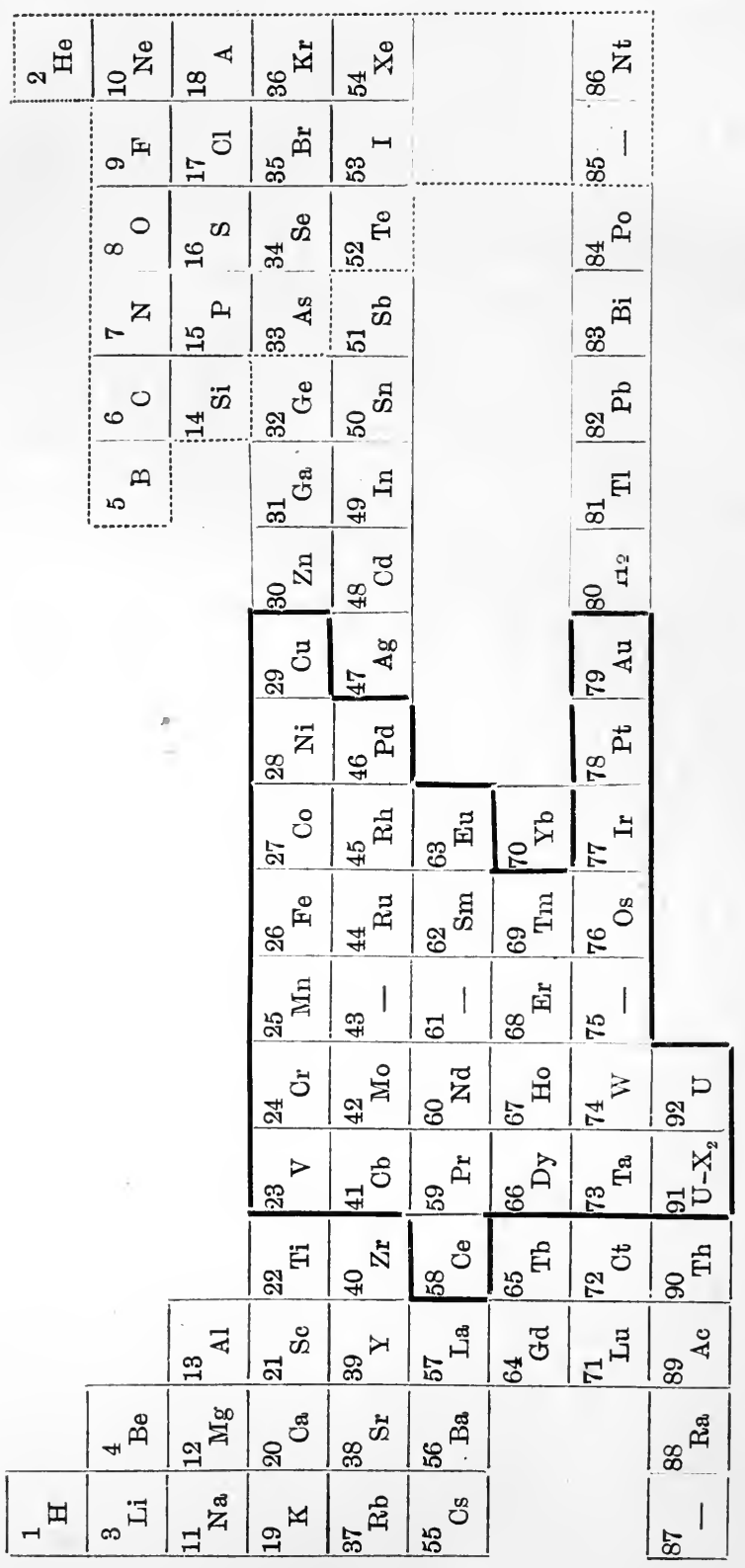

苞 쿄 秀

of

总

밈

의 며

ฐ.

응

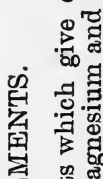

요용 क्ष. ఫ्वें 웡 ๘ँ कू \% 망 os 0 혔항 멸 


\section{The Theory of Meta-elements.}

The acceptance of Crookes' views on the composition of the materials which we term elements has been forced upon us by the experimental proof that isotopes exist; but up to the present no attempt seems to have been made to follow out to its logical conclusion the train of reasoning which is suggested by this result. We have accepted the idea that a substance may respond to all our chemical tests as if it were homogeneous in nature and yet may consist of a mixture of different kinds of atoms in arbitrary proportions. Indeed, if the newer results of positive ray analysis be confirmed, we shall have to admit that the chemical element chlorine, for instance, actually can be separated into two forms of matter each of which is different from the other although neither of them is simpler than "chemical" chlorine. Thus as far as the factor of mass is concerned, we have made a definite breach in the old definition of elements; and it now behoves us to examine other factors equally carefully in order to see whether they are also not subject to similar variations from the normal.

Next to mass, the electrical character of an atom appears to be of the greatest interest. Just as in earlier days we were satisfied that atomic weights were physical constants, so now we assume that the electromotive force of an element is an easily definable quantity. But if what we term elements are really congeries of non-homogeneous atoms, it seems clear that each of these atoms will have its own E.M.F.; so that the quantity actually measured in practice is an average value which may not correspond in practice to the E.M.F. of any particular atom in the group. It seems not impossible that by a prolonged series of "fractional electrolyses" a separation of the atoms having different E.M.F.'s might be accomplished.* The main difficulties to be anticipated are not concerned with actual separation, but are associated, as far as can be foreseen, with the proof of the separation after it has been attained.

At first sight, spectra appear to offer a very accurate criterion of the homogeneity of matter; but the results found with ordinary and X-ray spectra in the case of the isotopes have

* An attempt of this kind has been begun in the author's laboratory. 
proved that spectra are not to be relied on as tests for the differentiation of a mixture from a homogeneous material. It is possible that a revision of our spectroscopic methods combined with a photometric examination of the relative brightness in the spectral lines might render the test more rigid; but at present we cannot properly assume that the resolving power at our disposal is sufficient to solve all the possible problems which the meta-elements open before us.

When we turn to the chemical properties of the elements, the poverty of our methods of separation cannot fail to strike us. In the main, we depend almost entirely upon three different processes for dividing one form of matter from its neighbours. Differences of solubility between elements or between their compounds are the commonest phenomena which we utilize in order to separate mixtures into their components ; but it is only necessary to consider the case of the rare earth group to see the difficulties which attend this method and the uncertainties into which it may lead us. Less usual is the method of separation by distillation, which also is a very crude process. Finally, we have the ordinary electrolytic modes of dividing one material from another. It must be admitted that on the chemical side our methods are hardly such as to offer any real satisfaction. They are quite sufficient for our everyday needs ; but when applied to the case of meta-elements they fail completely, as is proved in the case of the isotopic groups. To use Crookes' analogy, they make us believe that we have discovered a fixed star when actually we are dealing with a nebula.

It seems probable that the next great stride forward in our knowledge of matter may come about through the invention of new methods of separation. So far, we have been in the position of a statistician confronted with a digest of the Census figures. We are able to predict very accurately how things will behave en masse; just as the Census official can predict the probable birth-rate or death-rate for the coming year; but finer values escape our grasp. We can prophesy how many radioactive atoms in a given mass will disintegrate within a fixed period; but we cannot isolate these particular atoms from the remainder of the material before the breakdown takes place. 


\section{Conclusion.}

A survey has now been made of the main characteristics presented by the Periodic Law; and it may not be amiss to indicate here some of the properties of elements and compounds which are also periodic in their nature, since it is only by the consideration of the largest possible number of allied phenomena that we can hope to evolve some hypothesis which may shed light upon the causes underlying this mysterious grouping.

As can be seen from the diagram at the end of this volume, there is a regular periodicity in electrochemical character and magnetic properties. Ductility, malleability, and brittleness, all show similar fluctuations with change in atomic number. The spectra of the elements bear a certain relation to the Periodic Law. As has been shown above, the colours of metallic chlorides exhibit some regularities which can hardly be accidental. The refractivities of certain elements have been adduced $^{1}$ to show that in their case also there is periodicity. Hardness also appears to vary periodically throughout the series of elements. ${ }^{2}$ The periodicity of compressibility among the elements has already been mentioned in this chapter. Thermal coefficients of expansion are periodic in their nature aiso, as are thermal and electrical conducting powers. Boilingpoint and melting-point were shown by Carnelley ${ }^{3}$ to be periodic both for elements and for compounds.

From this formidable list, it will be seen that the Periodic Law contains material for endless speculation even apart from the fundamental problem of its origin. For instance, to choose two examples given by Lothar Meyer, ${ }^{4}$ it may be asked why conductivity for heat and electricity should go hand in hand with ductility and malleability; or again, why the empirical rule should hold good that all elements possessing atomic weights less than forty obey Dulong and Petit's Law when

1 Cuthbertson and Metcalfe, Phil. Trans., 1907, 207, 135.

2 For Rydberg's table; see Landolt-Börnstein's Physikaltsch-chemische Tabellen.

3 Carnelley's papers are to be found in the Phil. Mag., 1879-1885; a summary, with references, is given in Chem. News, 1886, 53, 157, 169, 183, 197.

4 Lothar Meyer, Modern Theories of Chemistry, p. 144-5, 1888. 
their density is approximately less than one and a half times that of water?

Turning to another point, the differences between elements on "ascending" and "descending" branches of the atomic volume Curve attract attention. In the case of sodium and chlorine, the atomic volumes are equal, both elements are monovalent; and yet they differ entirely in properties. The only difference exhibited by the graph is the difference in atomic number (or number of positive charges in the nucleus); and it seems probable that some thought might profitably be expended in considering how this factor enters into the problem.

Again, the valencies of the elements in the eighth group of Mendeléeff's Table differ among themselves in a peculiar manner. Thus iron in the ferrates has a valency of six; cobalt forms an oxide in which the metal is quadrivalent; and nickel also seems to exert a maximum valency of four in its oxide. In the series ruthenium, rhodium and palladium the metals in the highest oxides obtained appear to be respectively octovalent, hexavalent and quadrivalent. Finally, osmium is octovalent; iridium is certainly quadrivalent and possibly reaches hexavalence; while platinum exhibits hexavalence. The differences seem worthy of further consideration, the more so since they form one of the greatest breaks in the symmetry of Mendeléeff's grouping.

Other points of interest will suggest themselves to any one who examines Periodic Law in detail; and it is possible that through such studies new light may be thrown upon the subject which may enable us to arrange the elements in a better order and thus finally be in a position to formulate some definite theory of the origin of the Periodic System. ${ }^{1}$

Whatever this theory may be, it will assuredly have to take into account the nature of the atomic nucleus, since this part of the elemental structure appears to be intimately connected with the atomic number and hence with the atomic order of the elements. It has been shown, in the foregoing pages, that the supposititious symmetry of the Mendeléeff system must be given up; and the new theory, when it is brought forward, will have to account for a much looser fabric. It seems not improbable

1 For suggestions of this type, see Carnelley, Chem. News, 1886, 53, 197 ; Urookes, ibid., 54, 114 ; Trans., 1888, 53, 487. 
that the key to the enigma may finally be discovered by a careful examination of the problems suggested by the rare earth elements, since they present in an exaggerated form the difficulties involved in the relations of the eighth group elements.

The work of recent years has brought us back to the point which Crookes reached in 1886. Until a year or two ago, we divided element from element by means of differences in chemical properties between the two; but with the newer knowledge which we now possess it seems as though we should have to accept Crookes' suggestion ${ }^{1}$ and take " not an external boundary, but an internal type" as our criterion. In the rare earth series, the chemical properties of the elements have so many points in common that it is hard to lay down a hard-andfast test of elemental character by purely chemical means; but the X-ray spectra appear to cleave a sharp line between one elementary form and its neighbours. Even here, however, the division is not a final one, since the isotopes have identical $\mathrm{X}$-ray spectra and yet we know from radioactivity measurements that there are fundamental differences between the members of this class. Even positive ray measurements will fail to separate from each other radium-D and the end-product of radium $-\mathrm{C}_{2}$, since these have the same atomic weight, although their stabilities are totally different.

Enough has been said to show the complexities before us when we attempt to define elemental matter; and it seems clear that unless we get some definite ideas on the subject we shall not be able to approach the problem of the Periodic Arrangement with any chance of solving it.

1 Crookes, Trans., 1888, 53, 490. 


\section{CHAPTER XX}

\section{CONCLUSION}

For close on thirty years the school of Ostwald dominated the field of physical chemistry; and it is now possible to take stock of the results attained by their efforts, and to draw a comparison between the hopes which appear to have inspired them and the reality achieved by their labours.

In the first place, it may be well to point out that most of the epoch-making discoveries had been made before this school came upon the scene at all. The Gas Laws, the Atomic Theory, the Law of Mass Action, Faraday's Law of Electrolysis, critical phenomena, van der Waals' equation, and the Kinetic Theory of Gases, to choose but a few examples, were all perfectly familiar to students of theoretical chemistry long before the name Physical Chemistry became current.

None the less, about 1887, when the Zeitschrift für physikalische Chemie was founded, the physical chemists of the time took up an attitude which, in the light of our present knowledge, appears rather pathetic. More by their manner of stating things, perhaps, than by actual affirmation, they gave the impression that physical chemistry, this "new" subject, was to regenerate the outworn fabric of the older science.

What has been the outcome of it all?

In the field of electrochemistry, a certain number of advances stand to their credit; but none of these can be placed upon a level with the greater generalizations which had already been made in the past. Let us take one or two examples of their achievements. The genius of van't Hoff evolved a theory of solution which agrees wonderfully with the facts within the limitations laid down by its author; but that theory has little or no application to the solutions which we commonly deal with in our test-tubes. Arrhenius cleared up many of our difficulties with regard to reactions in solution; but his theory leaves untouched practically the whole of organic chemistry 
After thirty years' incessant work, physical chemistry of the old-fashioned school has left us still devoid of any general theory of solution. Ostwald has given us a Dilution Law which breaks down completely when pressed beyond one single group of electrolytes; and his hypothesis as to indicators is chiefly notable on account of the fact that it missed the crux of the problem owing, probably, to his ignorance of the chemistry of the indicators themselves.* His attempts to dispense with the use of the atomic theory are now merely of antiquarian interest. $\dagger$

The condition into which physical chemistry had drifted towards the end of last century cannot be laid to the account of either van't Hoff or Arrhenius. Both of these men possessed strikingly original minds; and neither of them confined himself to a narrow groove in science. The leader upon whose shoulders the responsibility falls is Ostwald, the propagandist of the time. Under his guidance, physical chemistry degenerated to a great extent into a means of attacking the problems of pure chemistry instead of opening up new fields.

In seeking the reason for this decline, we have no need to travel far. Mathematics is a good servant, but a very bad master; and in the closing years of the physical chemistry which is now passing away, there can be little doubt as to its influence. It is quite true that the more a subject becomes amenable to mathematical treatment, the more accurate our knowledge of it becomes; but this is not quite the same thing as saying that the scientific value of a paper can be gauged from the number of signs of integration which it contains. Under the influence of Ostwald, numerical values assumed undue importance; and groups of facts which could not be expressed in quantitative terms were regarded in a somewhat step-motherly fashion.

Now, it is clear that, in the majority of cases, the first steps in a new field are qualitative rather than quantitative ones; so that by throwing undue importance upon quantitative values the breaking of new ground is discouraged to a greater or less

* This ignorance of organic chemistry appears to be a characteristic of the Ostwald school, few of whom have shown any grasp of the problems involved in the study of carbon compounds.

+ Even Ostwald himself has had to modify his attitude in this matter. See the Vorbericht to his Grundriss der allgemeinen Chemie, 4th edition, 1909. 
extent; and the incentive is given rather to the application of known methods to fresh cases. Perhaps it is not going too far if we see in this the origin of the sterility of the Ostwald school in the wider fields of physical chemistry.

Another trait of the older school of physical chemists seems to have been their dislike of the use of mechanical models. As any organic chemist knows, these mechanical conceptions are of the utmost value in many cases; but to the mathematically-minded physical chemist they appear to suggest nothing in particular. This is strange, since physicists of the rank of Clerk Maxwell have not despised their assistance. It seems probable that this contempt for mechanical illustrations accounts to a great extent for the failure of the Ostwald school in the field of organic chemistry. Ostwald himself suggested that organic compounds might be dealt with from the mathematical standpoint, and that their behaviour might be expressed, not in structural formulæ but by mathematical symbols. ${ }^{1}$ It is safe to say, however, that no results of any value whatever have been produced as yet; and the mechanical conception has justified itself by countless successes while the chemical mathematicians have produced nothing in this field which is worth the paper on which it was written. Yet the reactions of organic chemistry have been open to attack at any time within the last generation.

Lest there should be any misunderstanding it may be said quite plainly that the foregoing paragraphs are not intended to decry the value of mathematics when properly employed in chemical problems; but are meant merely to indicate that its function is a restricted one. Mathematics cannot be dispensed with unless we are prepared to abandon the development of the atomic theory, which depends upon quantitative relations; but when mathematics becomes anything more than a "brilliant second" in the chemical field the results are not likely to advance our knowledge. . Not only so, but the individual who uses mathematics as an adjunct to chemistry must have a real understanding of mathematical technique if the results are to be of any value. FitzGerald, in his Helmholtz Memorial Lecture, ${ }^{2}$ gave some examples of the pitfalls into which the

1 Ostwald, Zeitsch. physikal. Chem., 1908, 61, 507.

2 FitzGerald, Trans., 1896, 69, 885. 
mathematical smatterer may stumble; and it might be well were a wider circulation assured for the aphorism: "It is as risky for a chemist to apply mathematics as for a mathematician to lecture chemists."

In yet another direction, the influence of Ostwald upon his school has been equally unhappy. One of the most original of modern physicists ${ }^{1}$ once took the trouble to analyse Ostwald's views on science and, after some trenchant criticism, described them as " a sort of well-arranged catalogue of facts without any hypotheses . . . worthy of a German who plods by habit and instinct." It is doubtful if any unbiassed person would wish to enter the Ostwald school after a perusal of this examination of Ostwald's fundamental ideas. Hypotheses-and even theories-are, of course, ephemeral things; they serve the needs of their time and may pass away when we gain a fuller knowledge of facts. But a school which deliberately prefers to exclude hypotheses from its purview is certainly not likely to advance true knowledge to any great extent; though by drudgery and industry it may gather together a mass of experimental details.

It so happens that recent developments have thrown a flood of light upon the relative values of the two methods, considered as instruments of progress. Up to the last few years, the determination of atomic weights was a branch of chemistry in which the Ostwald method reached its culmination, for no hypotheses were employed and the facts were arranged, year by year, in the annual tables of atomic weights. Something like thirty different determinations seem to have been made of the atomic weight of lead; and among the experts there appears to have been a general agreement on a certain fixed value for the atomic weight. Suddenly, from the disintegration theory and especially from Soddy's far-reaching speculations, it became clear that the atomic weight of lead was not a physical constant. The application of an hypothesis and the subsequent testing of it overthrew at one stroke the whole value of previous investigations.

It might be contended by supporters of the "facts without hypothesis" school that, after all, established facts are of value in themselves. But the lead problem has shown that even this small grain of comfort is more than they are entitled

\footnotetext{
1 FitzGerald, Nature, 1896, 53, 441.
} 
to keep. It is now known that the elements are often mixtures of more than one type of atom; so that the whole of the laborious researches which have been devoted to " determining the atomic weights" prove in the end to be merely attempts to define the physical constants of a series of mixtures which may or may not be constant ones. What scientific value-or even interest-attaches to the fact that "lead" has an "atomic weight" of 207.095, when we now know that "lead" is in all probability a mixture of atoms having weights differing from each other by about one per cent.

Fortunately, the Ostwald school of physical chemistry is dying out; and a new and more hopeful change has come over the subject within the last few years. Some of the foregoing chapters have indicated the lines on which the fresh developments are proceeding; and they are only examples of the trend in this region of chemistry. We seem at last, after thirty years of relative stagnation, to be breaking fresh ground; and it may not be without interest to indicate briefly some of the points upon which our knowledge is still very incomplete.

One of the commonest facts in chemistry is that certain liquids will mix with one another whereas others do not mingle; yet our knowledge of the factors which govern these cases is very small. It is possible that the phenomena may be akin to "wetting" or "non-wetting"; but this brings us very little further forward.

The problem of non-ionic reactions such as esterification provides much material for speculation. The rapidity with which ionic reactions take place, even if no precipitation occurs, is extraordinary when we compare it with the comparative sluggishness characteristic of many organic reactions. The Law of Mass Action applies in both cases; so that the only factor left to us to account for the difference is chemical affinity. If we regard organic reactions as totally different from non-ionic ones, we are bound to inquire wherein the two sets differ; and the problem of the neutralization and esterification of the same acid provides a point not without interest.* If we assume that in the one case the matter is concerned with electrical charges, what are we to postulate in the case of organic reactions? Chemical affinity, according to

* Possibly we have to do in this case with differences of electric potential. 
modern views, is also electrical in character. Are we, then, to assume two different modes of electrical operation?

As regards the nature of concentrated solutions, we appear to be returning more and more towards the solvate hypothesis ; but the whole subject is still in a very obscure condition. ${ }^{1}$

The action of semi-permeable membranes is one which raises acute controversy. ${ }^{2}$ There are obviously three types of semi-permeable membrane : solid membranes, liquid membranes, and the boundary surfaces of solutions containing non-volatile solutes. No general theory has yet been proposed which includes all these cases.

One obvious factor in the problem appears to have been disregarded in modern views upon semi-permeable membranes : the velocity of the particles which leave the surface of the membrane. In order that a particle may be permanently withdrawn from any surface, the particle must attain a velocity greater than the critical velocity of the surface in question. If its velocity be lower than this critical velocity, it will fall back upon the surface. Applying this to the case of the semi-permeable membrane, it is evident that molecules travelling with a high velocity will escape, whilst those endowed with lower velocities will be retained.* If we assume that solute molecules are always associated with a large number of solvent molecules, this furnishes a simple explanation of the action of semi-permeable membranes in the case of solutions; for the friction upon the surface of the large solvate mass would lower its velocity considerably. Of course, in the case of aqueous solutions, since the solvent itself is associated, it is necessary to assume a very considerable association between solvent and solute. This agrees with the fact that in concentrated solutions the osmotic pressure is higher than the theoretical value; since on the solvate hypothesis much solvent is employed in forming solvate complexes and is thus prevented from acting as a solvent medium.

The solution problem may be approached from another

1 For an account of the evidence in favour of the solvate view, see Jones, The Nature of Solution (1917).

2 See Findlay, Osmotic Pressure (1913); and the discussion reported in Trans. Farad. Soc., 1917, 13, 119.

* $C f$. the speeds of hydrogen and nitrogen molecules in the case of a palladium membrane. 
point of view. At ordinary temperatures and pressures, solid sodium chloride is below its boiling-point and its vapour pressure is small. Yet if we apply water to it, it passes, according to the osmotic theory of solution, into something akin to the gaseous condition. It is evident from this that by applying water to its surface we have produced an effect parallel to that which we would bring about if we reduced the pressure on the surface of the salt. The external pressure, however, is apparently the same as before, if not actually greater. How can we explain the effect? The simplest way seems to be to assume that the solvent exerts an attractive power upon the solute. Thus in the case of a dissolving substance we should have the following forces in action: the vapour pressure of the solute, the attraction of the soivent, and the osmotic pressure in the solution. The first two factors would co-operate and would oppose the third. Saturation would occur when the three forces came into equilibrium.

With regard to the effect of a solvent upon the intramolecular structure of a solute dissolved in it we know very little. indeed; and an increase in our knowledge of this field might lead to interesting results. The problem is one which, from its nature, can hardly be attacked except by physical methods, such as an examination of absorption spectra, magnetic rotation, refractivity, magnetic susceptibility or dispersive power.

These physical methods themselves, however, stand in considerable need of dispassionate examination. From the purely chemical side we have constructed a series of constitutional formulæ which represent to a great extent the chemical behaviour of compounds. From the physical side we have amassed a considerable amount of data. Instead of constructing models which will account for our physical results, we have simply adopted the chemical model in toto; and have thus possibly over-loaded the scale on the chemical side instead of holding the balance evenly between the two.

Among the numerous physical properties which have been examined, optical rotatory power is one of those which have gained least from our investigations during the last generation. We are able to state from an examination of the structural formula of a compound whether or not the substance 
can show optical activity; but beyond that our knowledge stops short. We cannot make even a rough guess at the value of the rotation of the substance.

Crystallography is a subject which, until quite recently, lay almost outside the purview of the average chemist, whose views on crystal forms were generally confined to "small, flat plates" or "pointed leaflets" or some such description. The intricacy of crystal classification and the demands made by the subject upon the "three-dimensional imagination" sufficed to keep most dilettanti at a distance.

In recent years, however, Federov has succeeded in reducing to a form available for reference the data which have been accumulated with regard to many substances; with the result that a new method of identifying compounds has been placed in our hands. Federov's index contains particulars of some ten thousand substances and in order rapidly to identify a crystal of any of these it is only necessary to take certain measurements of the crystal in question, an operation occupying not more than two hours. The reliability of Federov's method was shown by his identification of forty-eight out of fifty compounds which were presented to him unlabelled and which he was able to recognize by crystallographic measurements alone.

The usefulness of this new method of identification, especially in cases where only small quantities of material are available, requires no advertisement here. In its rapidity it far surpasses ordinary analytical processes; and it appears to yield results which, if they do not absolutely establish the identity of a compound, at least render its recognition a comparatively easy matter. ${ }^{1}$

Turning to the properties of the elements, we find that many of them have recently been detected in a display of what may conveniently be termed abnormal valency. The old and apparently well-tried dogma of the quadrivalence of carbon has sustained a severe shock by the discovery of a large number of compounds in which carbon atoms behave as if they were trivalent. Triphenylmethyl, $\left(\mathrm{C}_{6} \mathrm{H}_{5}\right)_{3} \mathrm{C}$, is the best-known case, but there are many others in which carbon atoms show an abnormal behaviour. Again, in the hydrides of boron the

1 Particulars of Federov's method are given in Ann. Reports. 1912, 9, 261 ; 1913, 10, $245 ; 1914,11,248 ; 1917,14,230$. 
element acts as if it were quadrivalent. Divalent and quadrivalent nitrogen atoms have been detected in certain compounds. Sulphur and oxygen appear to act in certain cases as though they were monovalent; and the lead analogue of triphenylmethyl has been isolated. ${ }^{1}$

The whole problem of valency, ${ }^{2}$ however, bristles with difficulties; and we still await some hypothesis which will bring complete illumination upon it.

Why, for example, since we get $\mathrm{PCl}_{3}$ and $\mathrm{PH}_{3}$, should we not expect to produce $\mathrm{PH}_{5}$ just as easily as $\mathrm{PCl}_{5}$ ? Why is $\mathrm{PH}_{4} \mathrm{Cl}$ stable when $\mathrm{PHCl}_{4}$ has not been isolated? How comes it that when sulphur combines with four chlorine atoms the resulting compound is not salt-like; whereas the other form of quadrivalent sulphur, in the sulphonium salts, yields ions? Why is $\mathrm{H}_{2} \mathrm{~S}$ acidic in solution whilst $\mathrm{NH}_{3}$ is basic and $\mathrm{B}_{2} \mathrm{H}_{6}$ is unstable under the same conditions? Is there any connection between these facts and the capacity of ammonia to yield $\mathrm{NH}_{4} \mathrm{Cl}$ as well as $\mathrm{N}\left(\mathrm{C}_{2} \mathrm{H}_{5}\right)_{4} \mathrm{Cl}$, whilst sulphur will give rise to $\left(\mathrm{C}_{2} \mathrm{H}_{5}\right)_{3} \mathrm{~S}$. Cl but not to $\mathrm{H}_{3} \mathrm{~S} . \mathrm{Cl}$ ? How can we explain the fact that while $\mathrm{NH}_{3}, \mathrm{NCl}_{3}$, and $\mathrm{BCl}_{3}$ are known, the hydrogen compound of boron exists as $\mathrm{B}_{2} \mathrm{H}_{6}$ ? What forces retain the iron, carbon, and nitrogen atoms in the ferrocyanide ion and what relation do these forces bear to ordinary valency? Why, when a hydrogen atom is directly replaceable by a metallic atom, is the atom to which the hydrogen is attached always in a valency stage lower than that of its maximum valency? Many such questions will suggest themselves; and yet we have no clear answers to them.

Enough has now been said to show that there is much fresh ground still awaiting investigation even among the commonest facts of chemistry; and if the foregoing paragraphs have suggested anything of interest to the reader, they have amply fulfilled the object with which they were written.

1 See Stewart, Recent Advances in Organic Chemistry (4th edition), for an account of these cases.

${ }^{2}$ See Friend, The Theory of Valency; also Loring, Studies in Valency; Martin, Researches on the Affinities of the Elements; Nelson and Falk, School of Mines Quarterly, 1900, 30, 179; J. Amer. Chem. Soc., 1915, 37, 274; Nelson, Beans, and Falk, ibid., 1913, 35, 1810; Falk and Nelson, ibid., 1910, 32, 1637 ; 1911, 33, 1140; Falk, ibia., 1912, 34, 1041 ; Noyes, ibid., 1912, 34, 663; Fry, ibid., 1912, 34, 664; 1914, 36, 248, 262, 1035; 1915, 37, 885; 1916, 38, 1323, 1327, 1333; Falk and Nelson, Science, 1917, 46, 551. 


\section{APPENDIX}

\section{NOTES ON THE CONSTRUCTION OF THE PERIODIC SURFACE}

The model of the Periodic Surface is most easily constructed in the following way. Plot the Atomic Volume Curve on squared paper, placing the elements at a distance of one inch from each other, and making ten units of atomic volume equal to an inch and a half. Divide the paper at the points occupied by the following elements : hydrogen, helium, beryllium, neon, aluminium, argon, krypton, xenon, europium, ytterbium,* niton. Paste these various sections upon three-ply wood (or fret-wood), each piece of wood being seventeen inches in length. In the case of hydrogen and helium, leave sixteen inches in the centre of the wood slip ; leave ten inches between beryllium and boron; and ten inches between aluminium and silicon ; leave two inches blank before gadolinium and lutecium, nine inches blank after europium and ytterbium, and twelve inches blank after uranium.

Now plot on squared paper the atomic volumes of hydrogen, lithium, sodium, potassium, rubidium, cæsium, leaving an inch and a half between each element and reckoning, as before, that an inch and a half represents ten units of atomic volume. Four and a half inches beyond cæsium, introduce a point to represent the undiscovered alkali metal, No. 87 , which should be given the volume which seems best by extrapolation. Repeat the same process in the case of the elements helium, neon, argon, krypton, and xenon. Four and a half inches beyond xenon, insert the appropriate point for niton, and one and a half inches beyond niton, insert a point for No. 104. Paste these two graphs on wood also.

The various sections are now cut out by means of a fretsaw. In the case of the gaps between hydrogen and helium, beryllium and

* The volumes of most of the rare earths must be filled in as best one can as very few of them have been experimentally determined. 
boron, etc., leave a small tongue of wood, so as to make each series a complete piece.

Set up the two end-pieces containing the alkali group and the inert gas group and connect them at the appropriate point with the longer pieces, each of which is a section of the curve. Fasten them all together with sprigs or fine tacks.

The box-shaped arrangement thus produced is then fastened to a base-board seventeen inches by thirteen and a half, which it just covers.

Plaster of Paris is now poured into all the sections until it just overflows the upright divisions. The modelling of the higher parts of the curve is best done with material which has just begun to set. This method makes the model rather heavy to handle, and if lightness is required, the lower parts of the spaces can be filled up with papier maché made from macerated newspaper with a finish of plaster of Paris on the top; but the moist papier maché is apt to warp the wooden divisions. While the plaster of Paris is drying, it can be smoothed down with a putty-knife, and the curves of the surface justified.

The whole model is now coated with white paint; and black paint is applied to those spaces in which no elements occur. A blue line can be painted in on the surface to divide the non-metallic elements from the others ; it should lie as shown by the dotted line on p. 260 of this volume. The region of elements yielding coloured salts can be indicated either by drawing a red line round it, as shown by the heavy line on p. 260 , or by washing the whole region with a faint tint. The former method is better, as it permits the colouring of each square with the colour of the corresponding chloride, thus bringing out the relation between the salt-colours in the system.

Finally, pins are inserted in the fretwood divisions at the proper points to represent the elements, and labels are glued to the pins. These labels are best prepared by punching discs from thin millboard, by means of a large cork-borer and a hammer. The symbols of the elements are written on the discs with Indian ink.

In order to illustrate the physical properties of the families, a glass case is made to contain the model, and small labels are pasted on the inside of the top at the appropriate places, to indicate whether the elements are malleable or brittle, fusible or refractory, etc. 


\section{NAME INDEX}

Acheson, 6

Acker, 13, 14

Adams, 241

Allen, 121, 122

Ampère Electrical Company, 30

Andrade, 114, 120

Arbuthnot, 75

Arhle, 37

Armstrong, 60

Arrhenius, 266

Ashcroft, 14, 15

Aston, 214, 217

BABOROvsKy, 46

Baeyer, 40, 43

Baker, 56, 164

Baly, 64, 77, 78

Barkla, 91, 94

Bary, 163

Baur, 254

Baxter, 211

Beans, 274

Beatty, 93

Becquerel, 154, 155, 163, 164, 165, 167

Bender, 156

Benedicks, 253

Bennet, 50

Benoist, 95

Bernsthen, 26

Berthelot, 29, 46

Berzelius, 135

Beyer, 19

Bielecki, 85

Biltz, 254

Birkeland, 23

Blumenfeld, 133

Bohr, 117, 226

Bose, 46

Bosshard, 51

Boyle, 179, 236

Bragg, 102, 106, 113

Branch, 227

Brauner, 46, 253, 254

Bray, 227

Bredig, 38

Broek, 117, 227
Broglie, 114

Brooks, 179

Brühl, 38, 72

Bruhat, 50;

Büchner, 174

Bumstead, 179

Bunsen, 29

Cameron, 183, 191, 192

Campbell, 174

Carnelly, 251, 263, 264

Caro, 29

Castner, 13, 15

Chancourtois, 250

Chapman, 92, 93, 217

Collie, 111, 149, 151, 182, 193, 195, 196,213

Collier, 94

Colson, 31

Comte, 56

Constam, 47, 50

Cowles, 1

Cranston, 173, 174

Crookes, 18, 19, 90, 141, 154, 162, 167, $207,208,214,236,238,250,261$, $262,264,265$

Crowther, 141, 147

Crymble, 70, 73, 75, 78, 82

Curie, M., 211

Curie, Mme., 156, 158, 160, 163, 164, 185,192

Curie, P., 96, 157, 164, 179, 182, ‘'185, 188

Cuthbertson, 263

Danne, 179

d'Ans, 46, 47

Debierne, 158, 178, 182, 185, 188

Desch, 64, 78

Desfosses, 29

Deville, 31

Dewar, 188

Dobbie, 18, 74, 83, 84

Domcke, 56

Donkin, 24

Dony-Henault, 37 
Dorn, 172, 179

Duane, 151

Dubois, 50

Ebler, 156, 174

Edminson, 73

Egerton, 195

Elöd, 56

Engelhardt, 70

Ercolini, 45

Exner, 200

Eyde, 23

Fajans, 204, 205

Falk, 274

Faraday, 266

Federov, 273

Findlay, 271

Finkelstein, 224

FitzGerald, 268, 269

Fleck, 201, 202, 203, 204, 205, 208, 217,231

Foerster, 19

Fowler, 55

Fownes, 29

French, 30

Friederich, 46, 47

Friedrich, 102, 105

Friend, 124, 128, 132, 274

Friman, 114

Fry, 273

GaNs, 33

Garrett, 241

Geiger, 156, 175, 176, 226, 235

Giesel, 183, 188

Gimingham, 178

Girod, 2

Glasson, 93

Gleditsch, 192

Glendinning, 73

Goldstein, 90

Gran, 19

Grant, 181

Gray, J. A., 161

Gray, R. W., see Whytlaw-Gray.

Grover, 211

Gruszkiewicz, 23

Guldberg, 19

HABER, 19, 26, 28

Hackh, 241

Hahn, 173

Hall, 241, 250, 251, 252

Hansen, 47

Hardy, 164, 165

Harkins, 116, 211, 227, 241, 250, 251, 252

Hartley, Sir N., 64, 66, 67, 70, 74, 84, 87

Hartley, 217
Haschek, 200

Hauenstein, 43

Haughton, 241

Hemptinne, 24, 25

Henri, 85-87

Henriot, 174

Heroult, 2

Herringa, 46

Herweg, 114

Hevesy, 199, 200, 204

Hicks, 254

Hilditch, 73

Himstedt, 188

Hinsberg, 196

Hittorf, 224

Hofer, 19

Hoff, van't, 266, 267

Holmes, 190

Hönigschmid, 158, 210, 211

Hopkins, 241

Horovitz, 211

Horry, 7

Howles, 19, 22

Hoyermann, 29

Hulin-Schumann, 37

Hyman, 210, 212

JACKSON, 229

James, 131

Jantsch, 133

Jaubert, 50

Joly, 217

Jones, 271

KaIlan, 165

Kaiser, 28

Kaye, 91, 93, 112

Kelvin, 225

Kennedy, 174

Kingzett, 38

Klemensiewiez, 200

Knipping, 102, 105

Knox, 18, 19, 27

Kœnig, 19, 56

Kœnigsberger, 224

Körösy, 165

Kužma, 46

LABORDE, 157

Lambilly, 25, 26

Landauer, 152

Lantsberry, 196

Lauder, 83, 84

Laue, 102, 105

Le Alanc, 19

Lee, 19

Lembert, 211, 212

Le Rossignol, 26, 178

Levi, 45

Levin, 174

Lewis, G. N., 227 
Ley, 70

Lind, 165

Lindemann, 114, 176, 217

Lindt, 151

Little, 124, 128, 132

Loew, 251

Loring, 241, 274

Lowry, 58

Luther, 46 .

Lyman, 121

MACBETH, 70, 76

MacDougall, 19, 22

Mackenzie, 195

McLennan, 174

Magini, 74

Makower, 156, 179

Marsden, 196, 226

Marshall, 42, 45

Martin, 15, 17, 18, 133, 241, 274

Massini, 47

Masson, 195, 196

Maxwell, 268

Mayer, 225

Mehner, 31

Meitner, 173

Melikoff, 39, 41, 50

Mendeléef, 236, 237, 239, 240, 242, $243,244,251,252,253,255,264$

Merck, 37

Merton, 195

Metcalfe, 263

Meyer, L., 236, 237, 245, 246, 251, 263

Meyer, 188, 254

Migliorini, 45

Millikan, 225

Moissau, 1, 2, 4

Moore, 97

Moseley, 114-116, 118-120, 123, 255, 256

Mulder, 46

Müller, 224

Muthmann, 19, 126

NeLson, 274

Nernst, 19,22

Nicholson, 227

Norton, 17

Noyes, 227,274

Nüranen, 19

Nuttall, 175, 176, 235

O'NeILL, 29

Oordt, 26, 28

Ostwald, 266-70

Owen, 92

Owens, 177

Paneth, 199, 200, 216

Pascal, 73
Patterson, H. S., 149, 151, 193, 195, 196,213

Pauling, 23

Peltner, 48

Perkin, Sir W. H., 73

Perman, 164, 192

Pichou, 1

Pickering, 182

Pissarjewsky, 39, 41, 50

Playfair, 29

Pokorny, 46

Poole, 217

Pouzenc, 50

Precht, 158

Price, 42, 45

Pring, 15

Prout, 214, 236

RAMSAY, 24, 157, 158, 179, 180, 181, $182,183,184,187,188,191,192$, 197, 208, 232

Raschig, 46

Rayleigh, 19, 251

Rea, 73

Regnault, 24

Retgers, 254

Reychler, 254

Reynolds, 250

Richards, 211, 212, 242

Richardson, 225

Rideal, 3, 15

Riedel, 34

Rillet, 69

Röntgen, 154

Rossi, 200

Royds, 182, 192

Rudorf, 252

Ruer, 174

Runge, 158

Russ, 19

Russell, 200, 204, 205

Rutherford, 114, 117, 120, 160, 166, $168,177,179,182,185,186,187$, $192,196,197,226,229$

Rydberg, 116, 263

SADLER, 91, 94

Sagnac, 96

Schlundt, 156

Schlutius, 25

Schmidlin, 47

Schmidt, 241

Schöne, 40

Schönherr, 24

Schumann, 152

Schutzenberger, 31

Scott, 23

Serpek, 27

Siegbahn, 114, 211 


\section{Siemens, 1}

Smiles, 59, 72, 223

Soddy, 117, 118, 165, 166, 168, 170 , $171,173,174,176,177,182,183$, $184,187,-188,195,198,199,201$, $204,205,206,208,210,211,212$, $215,217,219,220,231,240,241$,

Soret, 69 $251,254,269$

Spencer, 124

Stackelberg, 241

Stansfield, 3, 15

Steele, 181, 254

Steinmetz, 241

Stenström, 211

Stewart, A. W., 70, 73, 74, 75, 76, 77, $78,81,220,229-33,250,274$

Stewart, J. Q., 233

Stintzing, 251

Stoney, 251

Strong, 174

Strutt, 53-8, 159, 161, 189, 195

Sudborough, 164

Šulc, 46

\section{TAFeL, 39}

Tanatar, 46, 48, 50

Tarugi, 45

Thiele, 71,72

Thomsen, 253

Thomson, Sir J. J., 141, 145, 148, 149, $150,151,152,195,213,225,226$

Thornton, 241

Thorpe, 158

Tiede, 56

Tinkler, 83, 84

Turrentine, 45
URBAIN, 125, 133

U.S.A. Bureau of Mines, 156

Usher, 164, 192

VAVON, 174

Verneuil, 254

Villiger, 40, 43

Vogel, 241, 253

W $\triangle A G E, 19$

Waals, van der, 266

Wadsworth, 211, 212

Warburg, 19

Watson, 46, 182

Watts, 158

Wegscheider, 216

Weiss, 126

Welsbach, 125, 135

Wendt, 151-2

Werner, 254

Wheeler, 179

Whiddington, 93, 97

Whytlaw-Gray, 158, 179, 180, 181

Willcock, 164

Willson, 7, 116

Willstätter, 43

Wilson, 227

Witt, 76, 77

Wöhler, 31

Wolff, 176

Wolffenstein, 39, 48

Wright, R., 70, 73, 75, 78, 81

Wright, 3, 15

Wyrouboff, 254

Young, 24

ZwICKY, 51 


\section{SUBJECT INDEX}

a-PARTICLE, 162, 169, 170, 201, 205

$\alpha$-ray change, 170, 174, 201-2

a-rays, 160, 162, 166, 167, 169

- deflection of, 161

- nature of, 162

- scattering of, 225

Absorption band, 64

- - " head " of, 64

- - "persistence" of, 65

Absorption curves, calculation of, 86

- curves, plotting of, $62 \mathrm{ff}$.

- general, see General Absorption

- X-rays of, $94 \mathrm{ff}$.

- selective, see Selective Absorption

- spectra, 59 ff.

- - and chemical change, 83

- - valency, $81 \mathrm{ff}$.

- - definition of, 60

- - Hartley method in, 66

- method of observing, $60 \mathrm{ff}$.

- spectrum of quinone, 62, 65

Acetone, spectrum of, 77

Acetyl-acetone, 78

Acetylene, 8, 29

Actinium, 172

Actinium-A, 174

- -B, 174, 205

$--\mathrm{C}, 174,206$

- $-\mathrm{D}, 174,207$

- X, 174, 198

Actinium emanation, 174

- series, 174, 178

Active deposit, 186

- nitrogen, action of, on elements and compounds, 54

- - effect of external influences on, 57

- - influence of impurities on, 55

- preparation of, 53

- - properties of, 54

- - spectrum of, 55

Activity, excited, 185

Additive properties, 59

Alkali halides, crystal structure of, 109

Alkali manufacture, 13

Alkyl halides, 70, 78
Aluminium nitride, 27

Alundum, 11

Ammonia, manufacture of, 24

Ammonium, 122

- hydroperoxide, 39

- persulphate, 42

"Antihypo," 49

"Aquadag," 6

Arc furnaces, 1, 22-3

Argon, 214

Asterium, 119

Astronomical elements, 120, 227

Atom, model, see Model Atom

Atomic core, 229

- nucleus, Moseley on, 118

- numbers and atomic weights, 117

- - - chemical sequence, 117, 119

- - definition of, 117

- positive nucleus, 226

- structure, $225 \mathrm{ff}$.

Atomic volume and valency, 243

Atomic volume curve, 237, 241 ff., 251

Atomic weight, 219, 220

- weight and X-rays, 92-7

- - nature of, 234

- not a constant, 210

- - of lead, 209, 270

B-RAY change, 170, 205, 222, 223

$\beta$-rays, 160, 161, 162

- and ionic charges, 208

- deflection of, 161

- velocity of, 162

Barium carbide, 30

Barium cyanamide, 30

- cyanide, 30

- hydroperoxide, 39

- peroxide, 40-1

Becquerel rays, $158 \mathrm{ff}$.

Becquerel rays, complexity of, $158 \mathrm{ff}$.

- - effects of, 164

- - ionising power of, 164

- physiological effects of, 165

Beer's Law, 64

Benzaldoximes, 74

Benzene system, 76, 111

Brassidic acid, 75 
Calcrum carbide, 6

Calcium cyanamide, 31

- fluoride crystal, 111

- hydride, 28

- hydroperoxide, 39

- nitrate, 23

- nitride, 28

- permutite, 35

- silicide, 11

Camphorquinone, 77

Canal rays, 80

Carbides, 6 ff, 30

Carbon disulphide, 12

Carborundum, 9

Caro's acid, 43, 44, 46

- constitution of, 44

Catalysis in nitrogen fixation, 26,28

Cathode rays, action of, on hydrogen, 193

- nature of, 90

- velocity of, 93

Celestial elements, 120

Chalcolite, 156

Chlorine isotopes, 214

Chlorosulphonic acid, 43

Chromium, 224

Chromogens, 77

Chromophores, 76

Citraconic acid, 73, 74

Cobalt, 224

Cobalt tri-electride, 232

Colligative properties, 59

Conjugated double bonds, $70 \mathrm{ff}$.

Constitutive properties, 59

Copper crystal, 112

Coronium, 119

Corpuscular rays, 96

Cotarnine, 83

Crookes' tube, 90

Crystallography, 273

Crystal structure, analysis of, $98 \mathrm{ff}$.

Cyanamide, 30

Cyanides, manufacture of, 28

"DAG," 6

Denitrifying bacteria, 17

Deposit, active, 186

Diacetyl, 77

Diactinic substances, 69

Diamagnetism, 73

Diamond crystal, 111

Diffraction, $98 \mathrm{ff}$.

- grating, crystal's action as, 104

Dilution Law, 267

Dimethyl-diacetylene, 76

Distintegration, multiple, 169

- of copper, 192

- of nitrogen, 196

— of thorium, 192

- theory, $167 \mathrm{ff}$.

Disintegrations, chart of, 207
Double bonds, conjugated, 72

Dowson gas, 25, 29

ELECTRIC furnace, $1 \mathrm{ff}$., $22 \mathrm{ff} ., 27,30$

- - arc type, 1

- - construction, 3

- - for alkali manufacture, 13

- - - alundum, 11

- - calcium carbide, 6

- - carbon disulphide, 12

- - carborundum, 9

_ - - fixation of nitrogen, $22 \mathrm{ff} ., 27$, 30

- - graphite, 5

- - - phosphorus, 12

- - - silicides, 10

- - - silicon, 8

- - - carbide, 9

- - essentials of, 3

- - ingot type of, 7

- - linings, 4

- - resistance type, 2

-- - rotary type of, 5,7

- - tapping type of, 7

-. - types of 1 , ff.

Electrides, 232

Electromotive force of atoms, 261

Electron as an element, 232

Electronic theories of atom, 225

Elementary groups, 238

Elements, analogy and identity of, 208

- " celestial," 120, 227

- homogeneous and heterogeneous, 216

- interpolated, 240

- passivity of, 224

- "pure "and " mixed," 216

Emanations, see Actinium, Thorium, and Niton

End-products of radioactive change, $171-3,210,216$

Equilibrium, radioactive, 169

Erucic acid, 75

Ether, luminiferous, 226

Ethyl iodide, 78-9

Ethylene iodide, 78-9

Excited activity, 185

FifTh-POWer Law, 92

Fixation of nitrogen, $16 \mathrm{ff}$.

Fluorescence produced by. Becquerel rays, 163

Fractional crystallisation, 131

Fumaric acid, 74

Furnace, electric, see Electric Furnace

$\gamma$-RAYs, $160 \mathrm{ff}$.

- nature of, 161

- origin of, 161

Geiger-Nuttall relation, 175, 235 
General absorption and conjugation, $70 \mathrm{ff}$.

- - unsaturation, $70 \mathrm{ff}$.

- - factors affecting, $68 \mathrm{ff}$.

- - of cyclic compounds, 70

- - homologues, 69

- - - isomers, $70 \mathrm{ff}$.

Geology and radioactivity, 189

Goldstein rays, 80

Graphite electric furnace, 5

Grating, diffraction, see Diffraction Grating

"Gredag," 6

H-PARTICLES, 196

Hartley method, 66, 85

Helium, 162, 169, 175, 187-9, 192, $193-5,213,214$

- - vacuum tubes, $193 \mathrm{ff}$.

- produced from niton, 187

- Collis-Patterson, work on, 193

- triatomic, $149 \mathrm{ff}$.

Heterobaric elements, 220

Heterotopic elements, 220

Hexatriene, 76

Hydrocyanic acid, 29

Hydrogen electride, 232

- peroxide, constitution of, 38

- - preparation of, 37

- - properties of, 38

- spectrum, 120

Hydroperoxides, 39

Hydroxycarbanil, $66 \mathrm{ff}$.

INCANDESCENT mantle, $135 \mathrm{ff}$.

- - branding, 138

- - burning, 140

- - collodionising, 140

- - fabrics, 138

- - fixing, 138

- - history of, 135

- - impregnation, 138

- testing, 140

Indicators, theory of, 267

Indium salts, 222

Indium's place in Periodic Table, 96

Interpolated elements, 240

Iodine, 78-9, 81

Iodoform, 78-9

Ionic and non-ionic reactions, 270

- charges and $\beta$-rays, 208

Ionium, 172, 198, 199, 206

- spectrum of, 200

Ions, absorptive power of, 82

Iron, ferrous and ferric, 123, $221 \mathrm{ff}$.

- E.M.F. of, 224

Isobarbituric acid, 87

Isobares, $218 \mathrm{ff}$., 233

- definition of, 219
Isobaric elements, see Isobares

Isotopes, 120, $198 \mathrm{ff}$., 218-9, 235, 238

- among common elements, 212

- atomic numbers of, 120

- - volume of, 212

- definition of, 199

- melting-points, 212

- separability of, 212,217

- spectra of, 120, 200, 211

- theory of, 198

- of hydrogen, 120

Isotope theory, tests of, 200

Isotopy, see Isotopes

Itaconic acid, 73

K-RAdiation, 92

Kanalstrahlen, 80

Krypton's isotopes, 214

L-RAdiation, 92

Lactam and lactim forms, 66

Lattice, see Space lattice

Laue's experiment, 105

Law, Periodic, see Periodic Law and Periodic Table

Lead, 120, 189, 209, 270

- atomic weight of, 209, 270

- and age of rocks, 189

Lightning, spectrum of, 182

Lyman spectrum of hydrogen, 121

M-RAdiation, 92

Magnesium di-electride, 232

- nitrides, 32

Magnetic rotation, 73

Magnets, Mayer's, 225

Maleic acid, 74

Manganese permutite, 35

Mantles, incandescent, see Incendescent mantles

Mass and electrical charge of atoms, 208,234

- of atoms and place in Periodic Table, 208, 215

Mass-spectra, 141

Membrane, palladium, 271

Membranes, semipermeable, 271

Mercury isotopes, 214

Mesaconic acid, 74

Mesothorium-1, 171, $221 \mathrm{ff}$.

$-2,171,221 \mathrm{ff}$.

Meta-elements, 207, 214, 236, $261 \mathrm{ff}$.

Metaneon, 213

Methyl iodide, 78-9

Methylene iodide, 78-9

Microbalance, 181

Minerals, age of, 189

Model atoms, $225 \mathrm{ff}$.

Model atom and radioactivity, 233

- - - rare earths, 231

Molecular numbers, 121 
Monazite sand, treatment of, $136 \mathrm{ff}$. Monox, 10

Multiple disintegration, 169

NEBulium, 119

Neon, 192, 195, 213

Net, 104

Nickel, 224

Niton, 172, 179 ff., 191-2, 201

- action on water, 183

- atomic weight of, 180

- disintegration of, 184

- heat emitted by, 182

- kinship with inert gases, 182

- liquefaction of, 180

- place in Periodic Table, 181

- properties of, 179

- spectrum of, 182

Nitre beds, 17

Nitric acid, manufacture of, $18 \mathrm{ff}$.

Nitrides, manufacture of, 31

Nitrifying bacteria, 17

Nitrogen, active form of, see Active nitrogen

- a third form of, 58

- circulation in nature, 16

- disintegration of, 196

- - as ammonia, 24

- - cyanides, 28

- - - nitrides, 31

- - oxides, etc., 18

Nitrolim, 31

Nitrous acid, manufacture of, $18 \mathrm{ff}$.

Non-ionic reactions, 270

" OrL-DaG," 6

Opacity, specific, 96

Optical rotatory power, 73 .

Osmotic pressure, 271-2

Palladium membrane, 271

Partial valencies, 71

Passivity, 224

Per-acids, $37 \mathrm{ff}$.

- definition of, 41

- elements forming, 41

- general character of, 41

Perborates, $50 \mathrm{ff}$.

Percarbonates, $47 \mathrm{ff}$.

Percolumbic acid, 47

Perdisulphuric acid, 41, 43-5

Periodic Law, 236 ff.

- properties of elements, 264

- Spirals, 250

- Surface, $251 \mathrm{ff}$.

- Table and radio-elements, 198 ff., 205-8

-_- anomalies in, 119, 238

- factors governing elements, places in, 208, 215

- gaps in, 118
Periodic Table, new form of, 260

Permonosulphuric acid, 41, 43-5

Permutites, $33 \mathrm{ff}$.

- in compound preparation, 36

- in water purification, $34 \mathrm{ff}$.

- removal of alkali by, 35

- - iron by, 35

Pernitric acid, 46

Peroxide of hydrogen, 37

Peroxides, metallic, 39

Perphosphoric acid, 46

Persulphates, $42 \mathrm{ff}$.

Persulphuric acids, $42 \mathrm{ff}$.

Pertantalic acid, 47

Pervanadic acid, 47

Phosphorus, manufacture of, 12

Pitchblende, 156, 211

Positive ray analyser, 141-2

- - analysis, $141 \mathrm{ff}$.

- - example of, 147

- - theory of, 143

- photographs, $145 \mathrm{ff}$.

- rays, 90, 141-153

Potassium halides, crystal structure of, $108 \mathrm{ff}$.

- perborate, 50

- percarbonate, $47-49$

- persulphate, 42

- radioactivity of, 174

- tri-iodide, 78-9

Precipitation, fractional, 130

Proto-elements, 120, 227

Protofluorine, 227

Protyle, 236

Psoudo-isobarism, 224, 233

QUARTz balance, 181

RadioactiniUm, 174, 198, 206

Radioactive change, end products of, 171-173, 210, 216

- constants, $171 \mathrm{ff}$.

- emanations, see Actinium, Thorium, and Niton

— equilibrium, 169

- recoil, 174

- series, $170 \mathrm{ff}$.

Radioactivity, disintegration theory of, 166

- history of, 154

- possible cause of, 233

- and geology, 189

Radio-elements and Periodic Table, $205 \mathrm{ff}$.

- chemistry of, 202

Radio-lead, 186

Radiothorium, 171, 198, 199, 223

Radium, 155 ff., 172, 185, 198, 201, $210,234,235$

- atomic weight of, 158

- chemical effects of, 164 
Radium, constants of, 172

- discovery of, 156

- emanation, see Niton

- metallic, 158

- physiological effects of, 165

- purification of salts of, 156, 157

- salts, properties of, 157

- series, 172

Radium-A, 172, 175, 186, 201

- - B , 120, 172, 175, 186, 202

- -C, 172, 186, 202, 215

$-\mathrm{C}_{1}, 172,216$.

- $\mathrm{C}_{2}, 173,210,216$

- D, 172, 186, 216, 223

- -E, 172, 186, 223

- F, 172, 186, 210, 223

Range of a particle, 176

Rare earth elements, 123, 124 ff., $253 \mathrm{ff}$.

- - - and model atom, 231

- - applications of, 133

- - atomic numbers of, 125

- - basicity of, 127

- - carbides of, 127

- - chemical character of, 126

- - classes of, 124-5

- - colours of chlorides, 257

- _ - double sulphates of, 129

- - fractional crystallisation of, 131

- - - precipitation of, 130

- - - hydrides of, 127

- - nitrides of, 127

- - number of, 118

- - oxides of, 127 255-7

- - purification of, 128

- - sources of, 125

- - - their place in Periodic Table, $123,252 \mathrm{ff}$.

Rays, $\alpha-, \beta-, \gamma$ - and $\chi$, see under $\alpha$, $\beta$, etc.

- cathode, see Cathode rays.

- corpuscular, 96

- positive, see Positive rays.

Reactions, ionic and non-ionic, 270

Recoil efficiency, 175

- radioactive, 174

Refractive index, 73

Refractory materials, 4

Residual affinity, 89

Resistance furnaces, 2

Röntgen rays and Becquerel rays, 161,163

Row, 104

Rubidium, radioactivity of, 174

Scattering of $\alpha$-particles, 225

Selective absorption, factors affecting, $76 \mathrm{ff}$.
Semipermeable membranes, 271

Series, radioactive, see Radioactive Series

Silicides, 10

Silicon, 8

- carbide, 9

- nitrides, 31

Siloxicon, 10

Silundun, 9

"Slice," 104

Sodium, manufacture of, 14

Sodium chloride, crystal structure of, 110

- hydroperoxide, 39

- percorate, 50

- permodocarbonate, 48

- permutite, 34-5

- peroxide, 40,50

- persulphate, 43

Soddy's Law, 203

Solution, nature of, 271, 272

Solvent action, 80

Space lattice, definition of, 103

Spectra, absorption, see Absorption spectra

- mass, see Positive ray

- of isotopes, 120, 200, 211

Spectrometer, X-ray, 106

Spectrophotometer, $84 \mathrm{ff}$.

Spectroscope, 60

Spectrum of hydrogen, 120

- - lightning, 182

Spinthariscope, 162

Structure of atom, see Atomic structure and Model atoms

Surface, periodic, $250 \mathrm{ff}$.

TANATAR's salt, 48

Tartaric acids, 74

Thorite, 210, 212

Thorium, 136, 137, 139, 168, 185, 198, $199,206,209,210$

- and uranous salts, 209

- emanation, 177, 185

- series, 171

Thorium-A, 171

- $-\mathrm{B}, 171,202-3$

- $-\mathrm{C}, 171,202-3$

- $\mathrm{C}_{1}, 170,171$

$-\mathrm{D}, 171$

Thorium-X, 168, 171, 198

Transmutation, $191 \mathrm{ff}$.

Transparency, equivalent, 96

Triketopentane, 77

Triphenylmethyl, 273

UNSATURATION and absorptive power, $70 \mathrm{ff}$.

Uraninite, 211

Uranium, 154, 155, 167, 172, 173, 189, 205, see also Uranium-1 
Uranium and age of rocks, 189

- salts,

$-1,172,173,209$

$-2,172,173,204,205,208,209,223$

- $\mathrm{X}, 167$

$-X_{1}, 172,173, ! 205,208,209,223$

$-\mathrm{X}_{2}, 172,173,204,223$

$--Y, 173,174$

$-Z, 173,174$

Uranium-radium series, 172

Uranous salts and thorium salts, 208, 231

Uric acid, 87

VALENCY, abnormal, 273

- and absorption power, $81 \mathrm{ff}$.

- problems, 273

WATER purification, 34

"Wetting," 270

$\mathrm{X}_{3}, 149 \mathrm{ff}$.

$\mathrm{X}$-ray and cathode ray energies, 93
X-ray spectra, 106, 114 ff., 215, 216, $239,255,265$

- spectra and atomic numbers, $114 \mathrm{ff}$.

- spectra of isotopes, 120

- spectrometer, 106

- spectrum of hydrogen, 120

- wave-length determination, 113

X-rays, 90 ff., 98 ff., 114 ff., 160, 161, 163

- absorption of, by elements, $94 \mathrm{ff}$.

- and atomic properties, $90 \mathrm{ff}$.

- and crystal structure, $98 \mathrm{ff}$.

- characteristic, $91 \mathrm{ff}$.

- emission of, by elements, $91 \mathrm{ff}$.

- " hard " and " soft," 92

- nature of, 91,92

- penetrating power of, 92

$\mathrm{Xenon}$, isotopes of, 214

Zeolites, 33

Zinc blende crystal, 111 


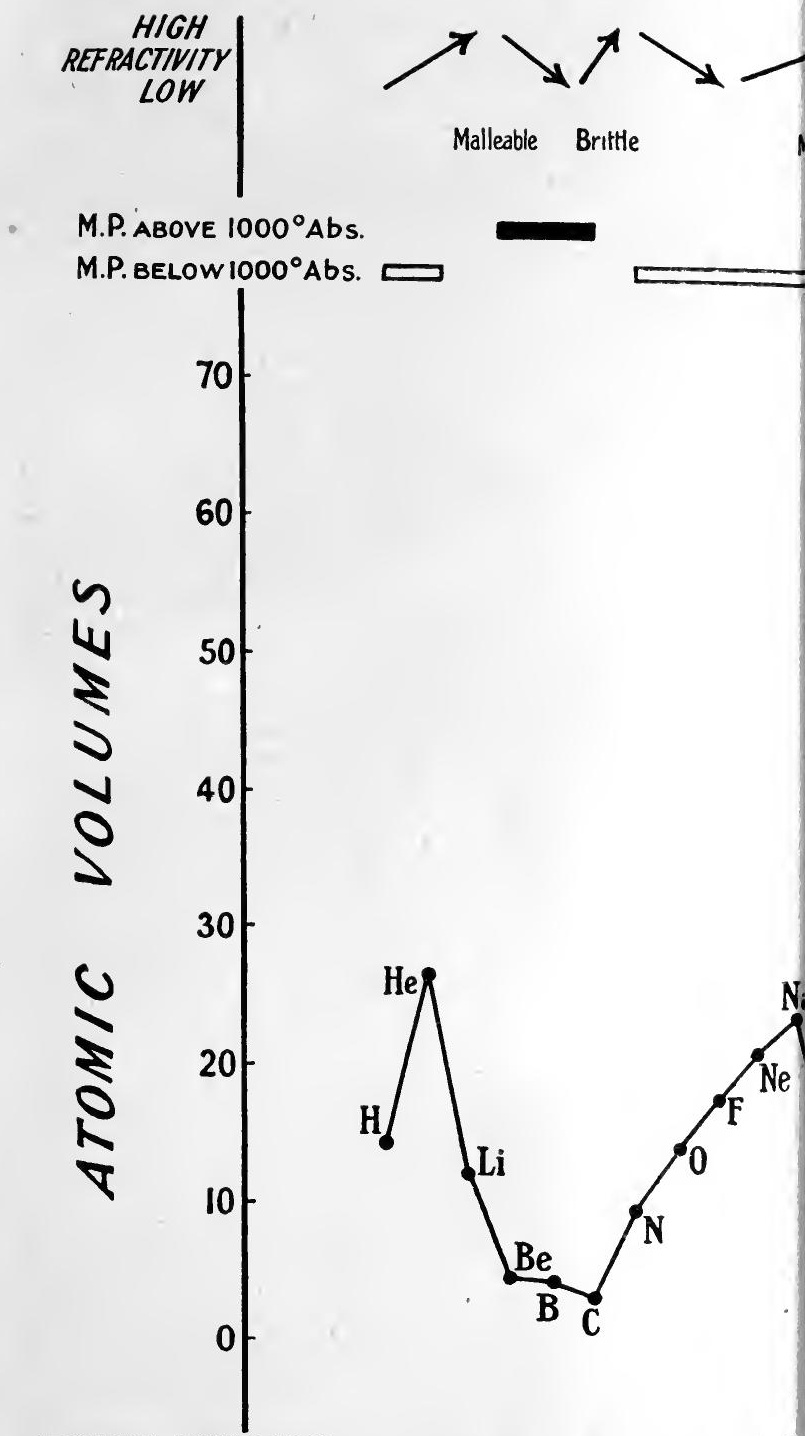

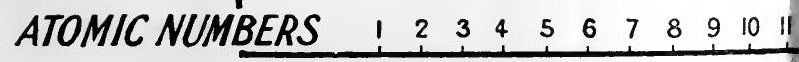

Electro positive

Hydroxides soluble in both acid and alkali

Electro negative 


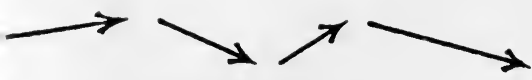

Malleable

Brittle

Malleable

Malle able

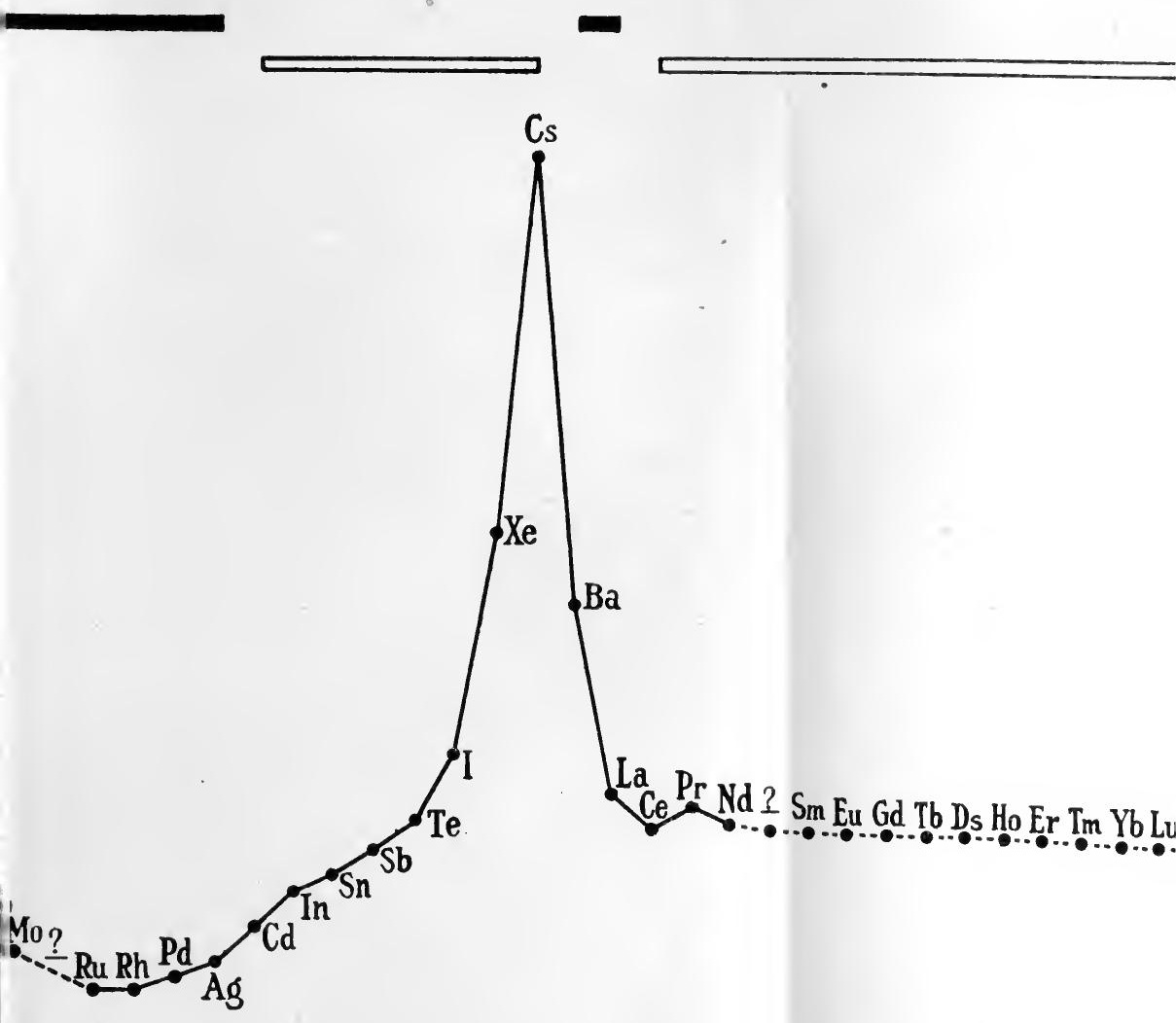

loured Salts

Coloured Salts

Col Salts

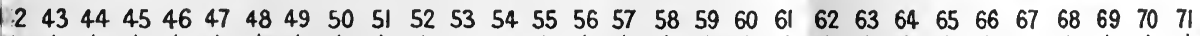




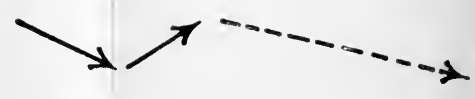

\section{Brittle Malleable}
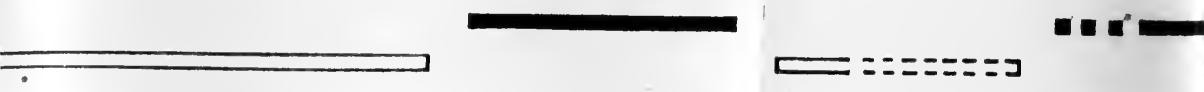

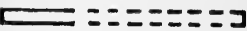

Eu Gd Tb Ds Ho Er Tm Yb Lu Ct Ta

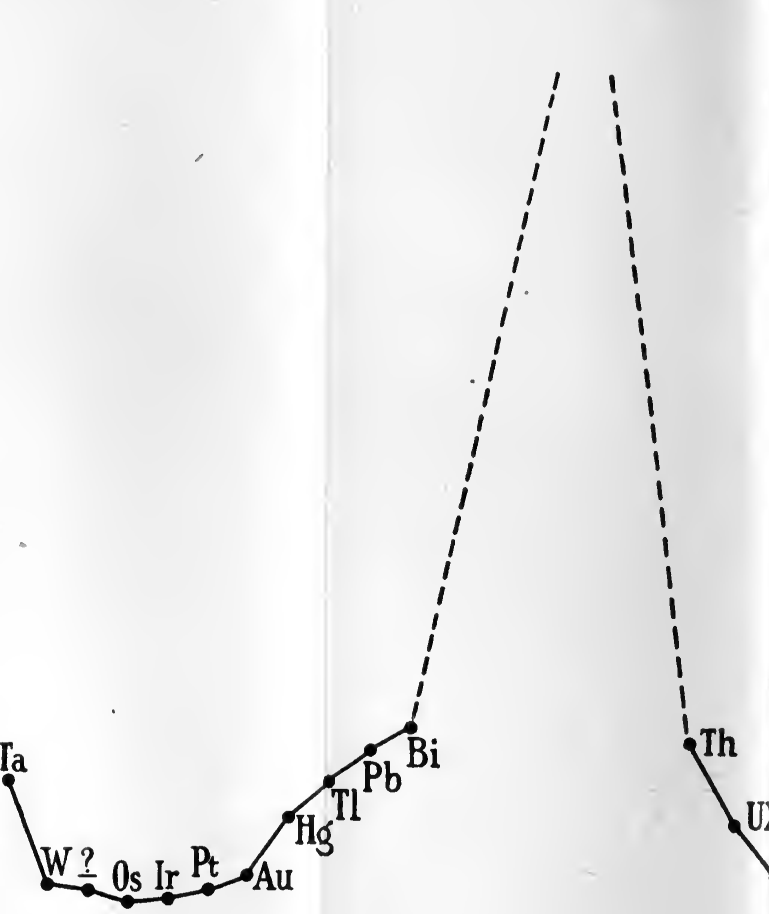





$-\therefore \quad \vdots$ 



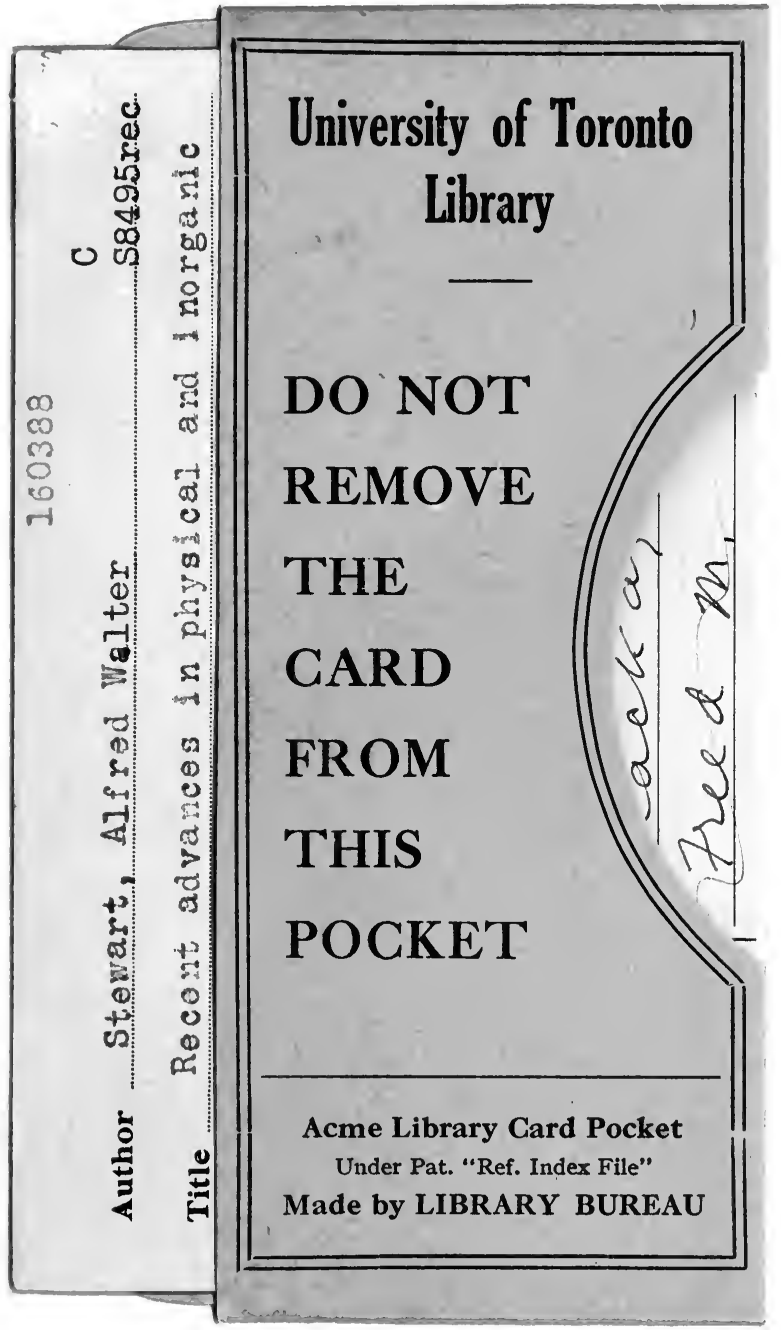


